Supporting Information

\title{
Enantioselective 1,2-Addition of $\alpha$-Aminoalkyl Radical to Aldehydes via Visible-Light Photoredox Initiated Chiral Oxazaborolidinium Ion Catalysis
}

Jae Yeon Kim, Yea Suel Lee, Yuna Choi, and Do Hyun Ryu*

Department of Chemistry, Sungkyunkwan University, Cheoncheon, Jangan, Suwon, 16419 (Korea)

Corresponding author : dhryu@ @sku.edu 


\section{Contents}

1. General Information $\quad$ S3

2. Preparation of Starting Materials

2.1 Preparation of Acrolein Derivatives $\quad$ S4

$\begin{array}{ll}\text { 2.2 Preparation of } \alpha \text {-Silyl Amines } & \text { S4 }\end{array}$

3. Preparation of $(S)$-Oxazaborolidinium Catalyst $\quad$ S6

4. General Procedure for Enantioselective $\boldsymbol{\alpha}$-Aminoalkyl Radical Addition $\quad$ S7

$\begin{array}{ll}\text { 5. Absolute Configuration Determination } & \text { S8 }\end{array}$

6. Characterization of Chiral $\boldsymbol{\beta}$-Amino Alcohol $\quad$ S9

7. Radical Trapping Experiment

$\begin{array}{ll}\text { 7.1 General Procedure for Radical Trapping Experiment } & \text { S27 }\end{array}$

7.2 Procedure for Asymmetric Roskamp Reaction in presence of TEMPO S27

$\begin{array}{lr}\text { 8. Radical Clock Experiment } & \text { S28 }\end{array}$

$\begin{array}{lr}\text { 9. Light On/Off Experiment } & \text { S30 }\end{array}$

$\begin{array}{ll}\text { 10. Quantum Yield Analysis } & \text { S31 }\end{array}$

11. Chemical Transformations of $\beta$-Amino Alcohols

11.1 Chemical Transformation of 5a to $8 \quad$ S33

$\begin{array}{ll}\text { 11.2 Chemical Transformation of } 5 \mathrm{~b} \text { to } 10 & \text { S34 }\end{array}$

$\begin{array}{ll}\text { 11.3 Chemical Transformation of } 6 \mathbf{k} & \text { S36 }\end{array}$

12. ${ }^{1} \mathrm{H}$ NMR and ${ }^{13} \mathrm{C}$ NMR Spectra $\quad$ S37

$\begin{array}{ll}\text { 13. HPLC Spectra } & \text { S85 }\end{array}$

$\begin{array}{lr}\text { 14. Reference } & \text { S132 }\end{array}$ 


\section{General Information}

Unless stated otherwise, reactions were performed in vacuum-flame dried glassware under a positive pressure of dry argon atmosphere using freshly distilled solvents. Commercial grade reagents were purified prior to use following the guidelines. ${ }^{1}$ Tetrahydrofuran and toluene were distilled from sodium wire. Dichloromethane was distilled from calcium hydride. Thin layer chromatography (TLC) was carried out on Merck silica gel 60 F254. Flash chromatography was performed using E. Merck silica gel (40-60 $\mu \mathrm{m}$ particle size). ${ }^{1} \mathrm{H}$ and ${ }^{13} \mathrm{C}$ NMR spectra were recorded on a Bruker at 500 and $125 \mathrm{MHz}$.

Chemical shift values are reported in ppm from tetramethylsilane as the internal standard. Data are reported as follows: chemical shift, integration, multiplicity $(\mathrm{s}=$ singlet, $\mathrm{d}=$ doublet, $\mathrm{t}=$ triplet, $\mathrm{dd}=$ doublet of doublets, $\mathrm{dt}=$ doublet of triplets, $\mathrm{m}=$ multiplet), and coupling constants $(\mathrm{Hz})$. Infrared spectra were recorded on a Bruker Vertex 70. HRMS were recorded on ESI-Q-TOF mass spectrometer (compact, Bruker Daltonics Inc.,Bremen Germany). LRMS data were obtained by Bruker Impact HD quadrupole time of flight. Analytical high performance liquid chromatography (HPLC) was performed on YL 9100 HPLC system using the indicated chiral column (Daicel Chiralcel, $4.6 \mathrm{~mm} \times 25 \mathrm{~cm}$ ). Optical rotations were determined on a Perkin-Elmer polarimeter model 343 plus at $589 \mathrm{~nm}$. 


\section{Preparation of Starting Materials}

\subsection{Preparation of Acrolein Derivatives}

(E)-3-(4-methoxyphenyl)-2-methylacrylaldehyde, ${ }^{2 \mathrm{a}}(E)$-3-(4-bromophenyl)-2-methylacrylaldehyde, ${ }^{2 \mathrm{a}}(E)-2-\mathrm{methyloct-2-}$ enal, ${ }^{2 \mathrm{~b}}$ 2,3-dimethylbut-2-enal, ${ }^{2 \mathrm{c}}(E)$-2-ethylidenehexanal, ${ }^{2 \mathrm{~d}}(E)$-2-benzylbut-2-enal, ${ }^{2 \mathrm{e}}$ cyclopent-1-enecarbaldehyde ${ }^{2 \mathrm{f}}$ and cyclohept-1-enecarbaldehyde ${ }^{2 \mathrm{~g}}$ were prepared according to a literature procedure.

\subsection{Preparation of $\alpha$-Silyl Amines}

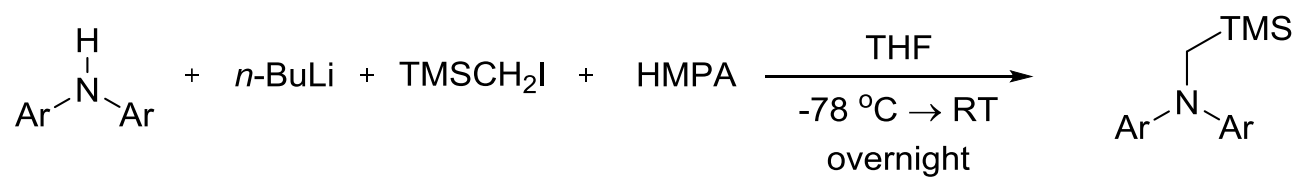

To a stirred solution of diarylamine (20 mmol, 1.0 equiv.) in THF $(50 \mathrm{~mL})$ and HMPA $(17 \% v / v, 8.5 \mathrm{~mL})$ was added $\mathrm{n}-$ $\operatorname{BuLi}\left(2.5 \mathrm{M}\right.$ in hexane, $12 \mathrm{~mL}, 30 \mathrm{mmol}, 1.5$ equiv.) dropwise over $10 \mathrm{~min}$ at $-78^{\circ} \mathrm{C}$. The reaction mixture was allowed to warm to room temperature and added (iodomethyl)trimethylsilane (7.4 mL, $50 \mathrm{mmol}, 2.5$ equiv.) slowly. The resulting solution was stirred overnight at room temperature. After completion of the reaction, the reaction mixture was quenched with distilled water $(50 \mathrm{~mL})$ and extracted with ethyl acetate $(3 \times 50 \mathrm{~mL})$. The combined extract was dried over anhydrous $\mathrm{Na}_{2} \mathrm{SO}_{4}$, filtered and concentrated in vacuo. The residue was purified by column chromatography (gradient elution with 0 $5 \%$ ethyl acetate-hexane).

\section{$N$-phenyl- $N$-((trimethylsilyl)methyl)aniline (2a)}<smiles>CCN(c1ccccc1)c1ccccc1</smiles>

Purification by column chromatography (gradient elution with hexane) afforded the desired product (90\% yield). Analytical data are consistent with reported values. ${ }^{3 a}$

${ }^{1}$ H NMR $\left(500 \mathrm{MHz}, \mathrm{CDCl}_{3}\right) \delta 7.25-7.22(\mathrm{~m}, 4 \mathrm{H}), 6.99-6.97(\mathrm{~m}, 4 \mathrm{H}), 6.92-6.89(\mathrm{~m}, 2 \mathrm{H}), 3.30(\mathrm{~s}, 2 \mathrm{H}),-0.05(\mathrm{~s}, 9 \mathrm{H})$

${ }^{13}$ C NMR (125MHz, $\left.\mathrm{CDCl}_{3}\right) \delta 149.7,129.2,121.2,121.1,43.8,-1.2$

4-Methoxy- $N$-(4-methoxyphenyl)- $N$-((trimethylsilyl)methyl)aniline (2b)<smiles>COc1ccc(N(C[Y5](C)(C)C)c2ccc(OC)cc2)cc1</smiles>

Purification by column chromatography (gradient elution with 5\% ethyl acetate-hexane) on activated silica gel $(2.0 \% \mathrm{v} / \mathrm{v}$ $\mathrm{Et}_{3} \mathrm{~N}$ in $n$-hexane) afforded the desired product ( $89 \%$ yield).

${ }^{1}$ H NMR $\left(500 \mathrm{MHz}, \mathrm{CDCl}_{3}\right) \delta 6.88-6.85(\mathrm{~m}, 4 \mathrm{H}), 6.82-6.79(\mathrm{~m}, 4 \mathrm{H}), 3.77(\mathrm{~s}, 6 \mathrm{H}), 3.14(\mathrm{~s}, 2 \mathrm{H}),-0.08(\mathrm{~s}, 9 \mathrm{H})$

${ }^{13}$ C NMR $\left(125 \mathrm{MHz}, \mathrm{CDCl}_{3}\right) \delta 154.3,144.5,122.4,114.6,55.7,44.4,-1.3$ 
IR $v_{\max } 3002,2950,2901,2830,1504,1463,1442,1240,1203,1180,1095,1038,967,856,838,759 \mathrm{~cm}^{-1}$.

LRMS (ESI): Calcd. for $\mathrm{C}_{18} \mathrm{H}_{25} \mathrm{NO}_{2} \mathrm{Si}: \mathrm{m} / \mathrm{z} 315.17\left([\mathrm{M}]^{+}\right)$, found: $\mathrm{m} / \mathrm{z}(\%)=315.16\left([\mathrm{M}]^{+}\right)$

\section{4-Bromo- $N$-(4-bromophenyl)- $N$-((trimethylsilyl)methyl)aniline (2c)}<smiles>CCN(c1ccc(Br)cc1)c1ccc(Br)cc1</smiles>

Purification by column chromatography (gradient elution with hexane) afforded the desired product (86\% yield). Analytical data are consistent with reported values. ${ }^{3 b}$

${ }^{1} \mathbf{H}$ NMR (500MHz, $\left.\mathrm{CDCl}_{3}\right) \delta 7.35-7.31(\mathrm{~m}, 4 \mathrm{H}), 6.85-6.82(\mathrm{~m}, 4 \mathrm{H}), 3.24(\mathrm{~s}, 2 \mathrm{H}),-0.03(\mathrm{~s}, 9 \mathrm{H})$

${ }^{13}$ C NMR $\left(125 \mathrm{MHz}, \mathrm{CDCl}_{3}\right) \delta 148.3,132.2,122.8,113.8,44.1,-1.1$

\section{$N$-(naphthalen-2-yl)- $N$-((trimethylsilyl)methyl)naphthalen-2-amine (2d)}<smiles>Cc1cc2ccccc2cc1N(C[As])c1ccc2ccccc2c1</smiles>

Purification by column chromatography (gradient elution with hexane) afforded the desired product (88\% yield).

${ }^{1} \mathbf{H}$ NMR $\left(500 \mathrm{MHz}, \mathrm{CDCl}_{3}\right) \delta 7.75-7.73(\mathrm{~m}, 2 \mathrm{H}), 7.68-7.67(\mathrm{~m}, 4 \mathrm{H}), 7.43-7.37(\mathrm{~m}, 4 \mathrm{H}), 7.34-7.30(\mathrm{~m}, 2 \mathrm{H}), 7.25-$ $7.23(\mathrm{~m}, 2 \mathrm{H}), 3.56(\mathrm{~s}, 2 \mathrm{H}), 0.03(\mathrm{~s}, 9 \mathrm{H})$

${ }^{13}$ C NMR $\left(125 \mathrm{MHz}, \mathrm{CDCl}_{3}\right) \delta 147.2,134.7,129.4,128.8,127.7,126.9,126.4,123.9,122.9,116.5,44.6,-0.9$ IR $v_{\max } 3055,3022,2955,2895,1623,1594,1573,1472,1395,1249,1226,1126,1052,962,820,741 \mathrm{~cm}^{-1}$.

LRMS (ESI): Calcd. for $\mathrm{C}_{24} \mathrm{H}_{25} \mathrm{NSiK}: \mathrm{m} / \mathrm{z} 394.14\left([\mathrm{M}+\mathrm{K}]^{+}\right)$, found: $\mathrm{m} / \mathrm{z}(\%)=394.14\left([\mathrm{M}+\mathrm{K}]^{+}\right)$

\section{9-((Trimethylsilyl)methyl)-9H-carbazole (2e)}

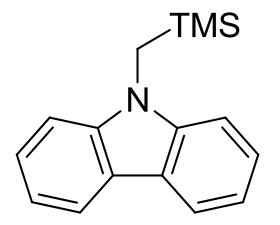

Purification by column chromatography (gradient elution with hexane) afforded the desired product (84\% yield). Analytical data are consistent with reported values. ${ }^{3 \mathrm{c}}$

${ }^{1} \mathbf{H}$ NMR $\left(500 \mathrm{MHz}, \mathrm{CDCl}_{3}\right) \delta 8.11(\mathrm{~d}, \mathrm{~J}=7.8 \mathrm{~Hz}, 2 \mathrm{H}), 7.46(\mathrm{ddd}, \mathrm{J}=8.2,7.2,1.1 \mathrm{~Hz}, 2 \mathrm{H}), 7.34(\mathrm{~d}, \mathrm{~J}=8.2 \mathrm{~Hz}, 2 \mathrm{H}), 7.21(\mathrm{ddd}$, $\mathrm{J}=7.8,7.2,1.1 \mathrm{~Hz}, 2 \mathrm{H}), 3.86(\mathrm{~s}, 2 \mathrm{H}), 0.07(\mathrm{~s}, 9 \mathrm{H})$

${ }^{13} \mathbf{C ~ N M R}\left(125 \mathrm{MHz}, \mathrm{CDCl}_{3}\right) \delta 140.8,125.5,122.6,120.3,118.4,109.0,34.4,-1.2$ 


\section{Preparation of $(S)$-Oxazaborolidinium Catalyst}

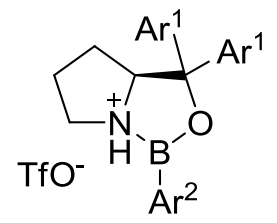

3a, $\mathrm{Ar}^{1}=$ Phenyl, $\mathrm{Ar}^{2}=$ Phenyl

3b, $\mathrm{Ar}^{1}=3,5$-Dimethylphenyl, $\mathrm{Ar}^{2}=$ Phenyl

3c, $A r^{1}=2,3$-Dimethylphenyl, $A r^{2}=$ Phenyl

3d, $\mathrm{Ar}^{1}=2,3-$ Dimethylphenyl, $\mathrm{Ar}^{2}=1$-Naphthyl

A $10 \mathrm{~mL}$ round-bottomed flask equipped with a stirring bar and a Dean-Stark trap (fully charged with activated $4 \AA$ molecular sieves) fitted on top with a reflux condenser and a argon inlet adaptor was charged with $(S)$-bis(2,3dimethylphenyl)(pyrrolidin-2-yl)methanol $(0.060 \mathrm{mmol})$, benzeneboronic acid $(0.060 \mathrm{mmol})$ and $20 \mathrm{~mL}$ of toluene. The resulting mixture was heated to reflux under argon atmosphere. After $3 \mathrm{~h}$, the reaction mixture was cooled to $c a .60{ }^{\circ} \mathrm{C}$ and the addition funnel and condenser were quickly replaced with a short-path distillation head. The mixture was concentrated by distillation (air-cooling) to a volume ca. $2.0 \mathrm{~mL}$. This distillation protocol was repeated three times by re-charging with $3 \mathrm{x}$ $5.0 \mathrm{~mL}$ of toluene. The solution was then allowed to cool to room temperature and the distillation head was quickly replaced with a vacuum adaptor. Concentration in vacuo $($ ca. $0.10 \mathrm{mmHg}, 0.5 \mathrm{~h})$ afforded the corresponding oxazaborolidine as clear oil.

To an aliquot of oxazaborolidine precursor $(0.060 \mathrm{mmol})$ in $1.0 \mathrm{~mL}$ of dichloromethane at room temperature was added triflic acid $(0.20 \mathrm{M}$ solution in dichloromethane, freshly prepared, $0.050 \mathrm{mmol}, 0.25 \mathrm{~mL})$ dropwise under argon atmosphere. After 15-20 min at room temperature, a homogeneous catalyst solution $\mathbf{3}$ was ready for use in enantioselective radical addition reaction. 


\section{General Procedure for Enantioselective $\alpha$-Aminoalkyl Radical Addition}

To a catalyst 3 solution prepared as described above in $1.0 \mathrm{~mL}$ of dichloromethane was successively added [ $\left.\operatorname{Ir}(\mathrm{dtbbpy})(\mathrm{ppy})_{2}\right]\left[\mathrm{BF}_{4}\right] 4$ (0.0025 mmol, 0.010 equiv.), aldehyde compound $\mathbf{1}$ (0.38 mmol, 1.5 equiv.) and $\alpha$-silyl amine compound 2 ( $0.25 \mathrm{mmol}, 1.0$ equiv.) at room temperature. The resulting mixture was irradiated with a $23 \mathrm{~W}$ compact fluorescent light bulb for $12 \mathrm{~h}$ at $35^{\circ} \mathrm{C}$. After completion of the reaction, the reaction mixture was quenched with $3.0 \mathrm{~mL}$ $\mathrm{K}_{2} \mathrm{CO}_{3} / \mathrm{MeOH}$ (saturated). After stirring for $10 \mathrm{~min}$ at room temperature, volatile materials were evaporated in vacuo and distilled water $(5.0 \mathrm{~mL})$ and ethyl acetate $(5.0 \mathrm{~mL})$ were added. The aqueous layer was extracted with ethyl acetate $(3 \mathrm{x} 5.0$ $\mathrm{mL})$. The combined extract was dried over anhydrous $\mathrm{Na}_{2} \mathrm{SO}_{4}$, filtered and concentrated in vacuo. The residue was purified by column chromatography (gradient elution with $1-17 \%$ ethyl acetate-hexane) to afford desired $\beta$-amino alcohol.

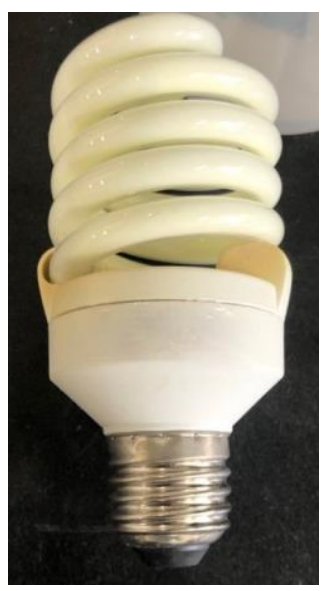

Figure S1 23 W Compact fluorescent light bulb
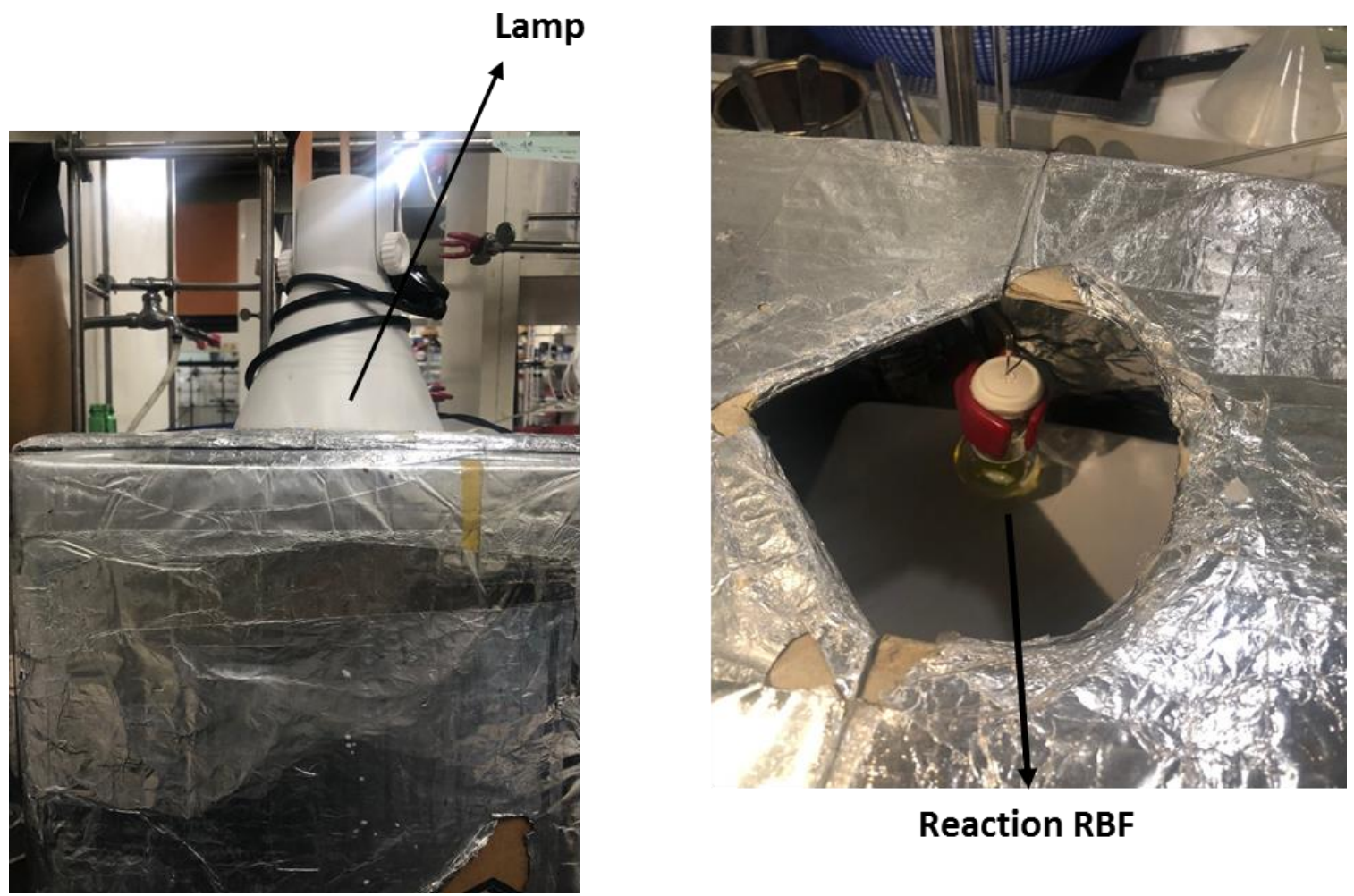

Reaction RBF

Figure S2 Reaction set-up of the photochemical reaction 


\section{Absolute Configuration Determination}

The absolute configuration of compounds $\mathbf{5 a}$ and $\mathbf{6 a}$ were confirmed by the modified Mosher ester method. ${ }^{4}$ Details of difference in chemical shifts $\left(\Delta \delta^{\mathrm{SR}}\right)$ for the diastereomeric MTPA esters are provided in the following structure. The absolute configuration of compound $\mathbf{5 b}$ was confirmed by the transformation to $\mathbf{1 0}$ and comparison of the optical rotation data with literature value. ${ }^{5}$

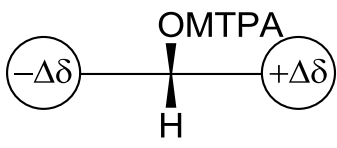

$$
\Delta \delta=\delta_{\text {S-ester }}-\delta_{\mathrm{R}-\mathrm{ester}}
$$

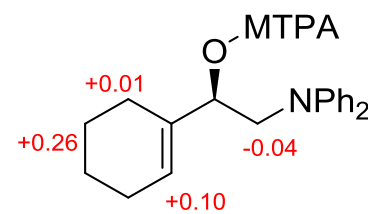

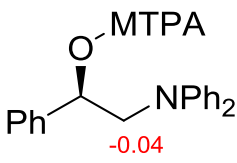




\section{Characterization of Chiral $\beta$-Amino Alcohol}

\section{(R)-1-cyclohexenyl-2-(diphenylamino)ethanol (5a)}<smiles>OC(CN(c1ccccc1)c1ccccc1)C1=CCCCC1</smiles>

Purification by column chromatography (gradient elution with 3-9\% ethyl acetate-hexane) afforded the desired product (68 $\mathrm{mg}, 93 \%)$ as colorless oil.

${ }^{1} \mathrm{H}$ NMR $\left(500 \mathrm{MHz}, \mathrm{CDCl}_{3}\right) \delta 7.25(\mathrm{dd}, \mathrm{J}=8.4,7.5 \mathrm{~Hz}, 4 \mathrm{H}), 7.04$ (d, J=7.7Hz, 4H), $6.97-6.94(\mathrm{~m}, 2 \mathrm{H}), 5.73$ (br s, 1H), 4.30 $(\mathrm{dd}, \mathrm{J}=8.5,4.1 \mathrm{~Hz}, 1 \mathrm{H}), 3.89(\mathrm{dd}, \mathrm{J}=14.8,4.2 \mathrm{~Hz}, 1 \mathrm{H}), 3.74(\mathrm{dd}, \mathrm{J}=14.8,8.5 \mathrm{~Hz}, 1 \mathrm{H}), 2.11-2.05(\mathrm{~m}, 4 \mathrm{H}), 1.88-1.84(\mathrm{~m}, 1 \mathrm{H})$, $1.59-1.55(\mathrm{~m}, 3 \mathrm{H}), 1.51-1.47(\mathrm{~m}, 1 \mathrm{H})$

${ }^{13} \mathbf{C}$ NMR $\left(125 \mathrm{MHz}, \mathrm{CDCl}_{3}\right) \delta 148.5,137.5,129.4,124.6,121.8,121.5,73.4,57.6,25.1,24.1,22.6,22.6$

IR $v_{\max } 3381,3060,3023,2858,1667,1496,1448,1363,1298,1246,1188,1138,1095,1028,991,848 \mathrm{~cm}^{-1}$.

HRMS (ESI, positive mode): Calcd. for $\mathrm{C}_{20} \mathrm{H}_{23} \mathrm{NONa}$ : m/z $316.1672\left([\mathrm{M}+\mathrm{Na}]^{+}\right)$, found: $\mathrm{m} / \mathrm{z} 316.1671\left([\mathrm{M}+\mathrm{Na}]^{+}\right)$ HPLC: $92 \%$ ee, ID, 2-propanol: $n$-Hexane $=5: 95$, flow: $1.0 \mathrm{~mL} / \mathrm{min}, 220 \mathrm{~nm}, T_{\mathrm{R}}=8.20 \mathrm{~min}$ (major) and $T_{\mathrm{R}}=9.10 \mathrm{~min}$ (minor).

$[\alpha]^{25} \mathrm{D}=+44.3\left(c=2.0, \mathrm{CHCl}_{3}\right)$.

(R)-2-(bis(4-methoxyphenyl)amino)-1-cyclohexenylethanol (5b)<smiles>COc1ccc(N(C[C@H](O)C2=CCCCC2)c2ccc(OC)cc2)cc1</smiles>

Purification by column chromatography (gradient elution with 5-17\% ethyl acetate-hexane) on activated silica gel (2.0\% $v / v \mathrm{Et}_{3} \mathrm{~N}$ in $n$-hexane) afforded the desired product (70 $\left.\mathrm{mg}, 79 \%\right)$ as yellow oil.

${ }^{1} \mathrm{H}$ NMR $\left(500 \mathrm{MHz}, \mathrm{CDCl}_{3}\right) \delta 6.96-6.94(\mathrm{~m}, 4 \mathrm{H}), 6.84-6.82(\mathrm{~m}, 4 \mathrm{H}), 5.75(\mathrm{br} \mathrm{s}, 1 \mathrm{H}), 4.22(\mathrm{dd}, \mathrm{J}=9.0,3.7 \mathrm{~Hz}, 1 \mathrm{H}), 3.78(\mathrm{~s}$, $6 \mathrm{H}), 3.75(\mathrm{dd}, \mathrm{J}=14.4,3.7 \mathrm{~Hz}, 1 \mathrm{H}), 3.60(\mathrm{dd}, \mathrm{J}=14.4,9.0 \mathrm{~Hz}, 1 \mathrm{H}), 2.17-2.10(\mathrm{~m}, 2 \mathrm{H}), 2.03-2.02(\mathrm{~m}, 2 \mathrm{H}), 1.91-1.86(\mathrm{~m}$, $1 \mathrm{H}), 1.63-1.58(\mathrm{~m}, 3 \mathrm{H}), 1.54-1.51(\mathrm{~m}, 1 \mathrm{H})$

${ }^{13}$ C NMR $\left(125 \mathrm{MHz}, \mathrm{CDCl}_{3}\right) \delta 154.8,142.9,137.5,124.4,122.8,114.8,73.2,58.6,55.7,25.1,24.2,22.6,22.6$

IR $v_{\max } 3504,3040,3003,2931,2858,1666,1596,1505,1464,1441,1241,1151,1036,919,895,823 \mathrm{~cm}^{-1}$.

HRMS (ESI, positive mode): Calcd. for $\mathrm{C}_{22} \mathrm{H}_{27} \mathrm{NO}_{3} \mathrm{Na}: \mathrm{m} / \mathrm{z} 376.1883\left([\mathrm{M}+\mathrm{Na}]^{+}\right)$, found: $\mathrm{m} / \mathrm{z} 376.1885\left([\mathrm{M}+\mathrm{Na}]^{+}\right.$)

HPLC: $93 \%$ ee, IC, 2-propanol: $n$-Hexane $=1: 9$, flow: $1.0 \mathrm{~mL} / \mathrm{min}, 220 \mathrm{~nm}, T_{\mathrm{R}}=8.27 \mathrm{~min}$ (major) and $T_{\mathrm{R}}=10.88 \mathrm{~min}$ (minor). $[\alpha]^{25} \mathrm{D}=+1.5\left(c=2.0, \mathrm{CHCl}_{3}\right)$.

(R)-2-(bis(4-bromophenyl)amino)-1-cyclohexenylethanol (5c) 
<smiles>OC(CN(c1ccc(Br)cc1)c1ccc(Br)cc1)C1=CCCCC1</smiles>

Purification by column chromatography (gradient elution with 2-9\% ethyl acetate-hexane) afforded the desired product (87 $\mathrm{mg}, 77 \%)$ as light yellow oil.

${ }^{1}$ H NMR (500MHz, $\left.\mathrm{CDCl}_{3}\right) \delta 7.37-7.34(\mathrm{~m}, 4 \mathrm{H}), 6.94-6.92(\mathrm{~m}, 4 \mathrm{H}), 5.73(\mathrm{br} \mathrm{s}, 1 \mathrm{H}), 4.26(\mathrm{dd}, \mathrm{J}=8.2,4.0 \mathrm{~Hz}, 1 \mathrm{H}), 3.83$ $(\mathrm{dd}, \mathrm{J}=15.0,4.2 \mathrm{~Hz}, 1 \mathrm{H}), 3.67(\mathrm{dd}, \mathrm{J}=15.1,8.4 \mathrm{~Hz}, 1 \mathrm{H}), 2.10-2.00(\mathrm{~m}, 3 \mathrm{H}), 1.86-1.84(\mathrm{~m}, 1 \mathrm{H}), 1.81$ (br s, $1 \mathrm{H}), 1.60-1.56$ $(\mathrm{m}, 3 \mathrm{H}), 1.54-1.46(\mathrm{~m}, 1 \mathrm{H})$

${ }^{13}$ C NMR $\left(125 \mathrm{MHz}, \mathrm{CDCl}_{3}\right) \delta 147.1,137.6,132.4,125.0,123.1,114.5,73.4,57.3,25.1,24.1,22.5,22.5$

IR $v_{\max } 3481,3018,2928,2858,1667,1580,1563,1488,1361,1246,1215,1137,1010,920,813,655 \mathrm{~cm}^{-1}$.

HRMS (ESI, positive mode): Calcd. for $\mathrm{C}_{20} \mathrm{H}_{21} \mathrm{Br}_{2} \mathrm{NO}: \mathrm{m} / \mathrm{z} 471.9882\left([\mathrm{M}+\mathrm{Na}]^{+}\right)$, found: $\mathrm{m} / \mathrm{z} 471.9886\left([\mathrm{M}+\mathrm{Na}]^{+}\right)$

HPLC: $89 \%$ ee, ID, 2-propanol: $n$-Hexane $=1: 9$, flow: $1.0 \mathrm{~mL} / \mathrm{min}, 220 \mathrm{~nm}, T_{\mathrm{R}}=5.48$ min (major) and $T_{\mathrm{R}}=6.56 \mathrm{~min}$ (minor). $[\alpha]^{25} \mathrm{D}=+73.2\left(c=2.0, \mathrm{CHCl}_{3}\right)$

(R)-1-cyclohexenyl-2-(dinaphthalen-2-ylamino)ethanol (5d)<smiles>O[C@@H](CN(c1ccc2ccccc2c1)c1ccc2ccccc2c1)C1=CCCCC1</smiles>

Purification by column chromatography (gradient elution with 2-9\% ethyl acetate-hexane) afforded the desired product (62 $\mathrm{mg}, 63 \%$ ) as yellow oil.

${ }^{1} \mathbf{H}$ NMR $\left(500 \mathrm{MHz}, \mathrm{CDCl}_{3}\right) \delta 7.72(\mathrm{~d}, \mathrm{~J}=8.1 \mathrm{~Hz}, 2 \mathrm{H}), 7.68(\mathrm{~d}, \mathrm{~J}=8.9 \mathrm{~Hz}, 2 \mathrm{H}), 7.65(\mathrm{~d}, \mathrm{~J}=8.1 \mathrm{~Hz}, 2 \mathrm{H}), 7.46(\mathrm{~d}, \mathrm{~J}=1.9 \mathrm{~Hz}, 2 \mathrm{H})$, $7.41-7.38(\mathrm{~m}, 2 \mathrm{H}), 7.33-7.30(\mathrm{~m}, 2 \mathrm{H}), 7.27(\mathrm{dd}, \mathrm{J}=8.9,2.0 \mathrm{~Hz}, 2 \mathrm{H}), 5.76(\mathrm{br} \mathrm{s}, 1 \mathrm{H}), 4.42(\mathrm{dd}, \mathrm{J}=8.2,4.0 \mathrm{~Hz}, 1 \mathrm{H}), 4.09(\mathrm{dd}$, $\mathrm{J}=15.0,4.2 \mathrm{~Hz}, 1 \mathrm{H}), 3.94(\mathrm{dd}, \mathrm{J}=15.0,8.4 \mathrm{~Hz}, 1 \mathrm{H}), 2.17-2.13(\mathrm{~m}, 2 \mathrm{H}), 2.00-1.90$ (m, 2H), $1.59-1.53(\mathrm{~m}, 3 \mathrm{H}), 1.49-1.45$ $(\mathrm{m}, 1 \mathrm{H})$

${ }^{13} \mathbf{C ~ N M R}\left(125 \mathrm{MHz}, \mathrm{CDCl}_{3}\right) \delta 145.9,137.7,134.6,129.7,129.0,127.6,127.0,126.4,124.7,124.3,123.0,117.0,73.2,57.9$, $25.1,24.2,22.6,22.5$

IR $v_{\max } 3492,3056,3018,2928,2858,1626,1596,1507,1471,1391,1271,1215,1186,1088,962,840 \mathrm{~cm}^{-1}$.

LRMS (ESI): Calcd. for $\mathrm{C}_{28} \mathrm{H}_{27} \mathrm{LiNO}$ : m/z $400.23\left([\mathrm{M}+\mathrm{Li}]^{+}\right)$, found: $\mathrm{m} / \mathrm{z}(\%)=400.26\left([\mathrm{M}+\mathrm{Li}]^{+}\right)$

HPLC: $91 \%$ ee, ID, 2-propanol: $n$-Hexane $=1: 9$, flow: $1.0 \mathrm{~mL} / \mathrm{min}, 220 \mathrm{~nm}, T_{\mathrm{R}}=8.98 \mathrm{~min}\left(\right.$ major) and $T_{\mathrm{R}}=10.06 \mathrm{~min}$ (minor).

$[\alpha]^{25}{ }_{\mathrm{D}}=+135.6\left(c=2.0, \mathrm{CHCl}_{3}\right)$.

\section{(R)-1-cyclopentenyl-2-(diphenylamino)ethanol (5e)}<smiles>O[C@H](CN(c1ccccc1)c1ccccc1)C1=CCCC1</smiles> 
Purification by column chromatography (gradient elution with 2-9\% ethyl acetate-hexane) afforded the desired product (35 $\mathrm{mg}, 50 \%)$ as colorless oil.

${ }^{1}$ H NMR $\left(500 \mathrm{MHz}, \mathrm{CDCl}_{3}\right) \delta 7.28-7.24(\mathrm{~m}, 4 \mathrm{H}), 7.06-7.04(\mathrm{~m}, 4 \mathrm{H}), 6.98-6.95(\mathrm{~m}, 2 \mathrm{H}), 5.69$ (br s, 1H), 4.59 (dd, $\mathrm{J}=8.8,3.0 \mathrm{~Hz}, 1 \mathrm{H}), 3.96(\mathrm{dd}, \mathrm{J}=14.8,3.6 \mathrm{~Hz}, 1 \mathrm{H}), 3.76(\mathrm{dd}, \mathrm{J}=14.8,8.9 \mathrm{~Hz}, 1 \mathrm{H}), 2.39-2.24(\mathrm{~m}, 4 \mathrm{H}), 2.14(\mathrm{br} \mathrm{s}, 1 \mathrm{H}), 1.88-$ $1.82(\mathrm{~m}, 2 \mathrm{H})$

${ }^{13}$ C NMR $\left(125 \mathrm{MHz}, \mathrm{CDCl}_{3}\right) \delta 148.5,144.2,129.5,127.2,122.0,121.6,68.7,57,9,32.4,31.7,23.4$

IR $v_{\max } 3462,3057,3022,2951,2927,1654,1559,1496,1467,1341,1246,1155,1058,992,896,864 \mathrm{~cm}^{-1}$.

LRMS (ESI): Calcd. for $\mathrm{C}_{19} \mathrm{H}_{21} \mathrm{NNaO}: \mathrm{m} / \mathrm{z} 302.15\left([\mathrm{M}+\mathrm{Na}]^{+}\right)$, found: $\mathrm{m} / \mathrm{z}(\%)=302.15\left([\mathrm{M}+\mathrm{Na}]^{+}\right)$

HPLC: $93 \%$ ee, OD-H, 2-propanol: $n$-Hexane=1:99, flow: $1.0 \mathrm{~mL} / \mathrm{min}, 220 \mathrm{~nm}, T_{\mathrm{R}}=11.93 \mathrm{~min}(\operatorname{minor})$ and $T_{\mathrm{R}}=13.16 \mathrm{~min}$ (major).

$[\alpha]^{25}=+43.3\left(c=1.0, \mathrm{CHCl}_{3}\right)$.

\section{(R)-1-cycloheptenyl-2-(diphenylamino)ethanol (5f)}<smiles>O[C@H](CN(c1ccccc1)c1ccccc1)C1=CCCCCC1</smiles>

Purification by column chromatography (gradient elution with 2-9\% ethyl acetate-hexane) afforded the desired product (41 $\mathrm{mg}, 53 \%)$ as yellow oil.

${ }^{1}$ H NMR $\left(500 \mathrm{MHz}, \mathrm{CDCl}_{3}\right) \delta 7.28-7.25(\mathrm{~m}, 4 \mathrm{H}), 7.05(\mathrm{~d}, \mathrm{~J}=7.8 \mathrm{~Hz}, 4 \mathrm{H}), 6.98-6.95(\mathrm{~m}, 2 \mathrm{H}), 5.88(\mathrm{t}, \mathrm{J}=6.4 \mathrm{~Hz}, 1 \mathrm{H}), 4.31$ (dd, J=8.8, 3.9Hz, 1H), $3.84(\mathrm{dd}, \mathrm{J}=14.9,4.0 \mathrm{~Hz}, 1 \mathrm{H}), 3.67$ (dd, J=14.8, 8.9Hz, 1H), $2.19-2.04$ (m, 5H), $1.75-1.68$ (m, $2 \mathrm{H}$ ), $1.50-1.43(\mathrm{~m}, 4 \mathrm{H})$

${ }^{13}$ C NMR $\left(125 \mathrm{MHz}, \mathrm{CDCl}_{3}\right) \delta 148.5,143.9,129.6,129.4,121.9,121.6,74.7,57.7,32.7,28.4,28.3,27.3,26.9$

IR $v_{\max } 3441,3060,3021,2921,2850,1668,1589,1496,1447,1361,1298,1246,1124,1072,965,852 \mathrm{~cm}^{-1}$.

LRMS (ESI): Calcd. for $\mathrm{C}_{21} \mathrm{H}_{25} \mathrm{NNaO}: \mathrm{m} / \mathrm{z} 330.18\left([\mathrm{M}+\mathrm{Na}]^{+}\right)$, found: $\mathrm{m} / \mathrm{z}(\%)=330.18\left([\mathrm{M}+\mathrm{Na}]^{+}\right)$

HPLC: $92 \%$ ee, ID, 2-propanol: $n$-Hexane=1:9, flow: $1.0 \mathrm{~mL} / \mathrm{min}, 220 \mathrm{~nm}, T_{\mathrm{R}}=5.88 \mathrm{~min}$ (major) and $T_{\mathrm{R}}=6.18 \mathrm{~min}(\mathrm{minor})$. $[\alpha]^{25}=+72.3\left(c=1.0, \mathrm{CHCl}_{3}\right)$.

\section{$(R, E)-1-(d i p h e n y l a m i n o)-3-m e t h y l p e n t-3-e n-2-o l ~(5 g)$}<smiles>C/C=C(/C)[C@@H](O)CN(c1ccccc1)c1ccccc1</smiles>

Purification by column chromatography (gradient elution with 2-9\% ethyl acetate-hexane) afforded the desired product (47 $\mathrm{mg}, 70 \%)$ as brown oil.

${ }^{1} \mathbf{H}$ NMR $\left(500 \mathrm{MHz}, \mathrm{CDCl}_{3}\right) \delta 7.26-7.23(\mathrm{~m}, 4 \mathrm{H}), 7.03(\mathrm{~d}, \mathrm{~J}=8.1 \mathrm{~Hz}, 4 \mathrm{H}), 6.96-6.93(\mathrm{~m}, 2 \mathrm{H}), 5.54(\mathrm{q}, \mathrm{J}=5.7 \mathrm{~Hz}, 1 \mathrm{H}), 4.32$ (dd, J=8.5, 4.1Hz, 1H), 3.84 (dd, J=14.8, 4.1Hz, 1H), 3.72 (dd, J=14.8, 8.7Hz, 1H), 2.11 (br s, 1H), 1.59 (s, 3H), 1.58 (d $\mathrm{J}=5.7 \mathrm{~Hz}, 3 \mathrm{H})$

${ }^{13} \mathrm{C}$ NMR $\left(125 \mathrm{MHz}, \mathrm{CDCl}_{3}\right) \delta 148.5,135.5,129.4,122.3,121.8,121.5,74.5,57.6,13.2,11.6$ IR $v_{\max } 3416,3061,3036,2975,2861,1671,1588,1495,1449,1363,1271,1216,1096,1028,888,846 \mathrm{~cm}^{-1}$.

HRMS (ESI, positive mode): Calcd. for $\mathrm{C}_{18} \mathrm{H}_{21} \mathrm{NONa}: \mathrm{m} / \mathrm{z} 290.1515\left([\mathrm{M}+\mathrm{Na}]^{+}\right)$, found: m/z $290.1516\left([\mathrm{M}+\mathrm{Na}]^{+}\right)$ HPLC: $91 \%$ ee, ID, 2-propanol: $n$-Hexane=5:95, flow: 1.0mL/min, 220nm, $T_{\mathrm{R}}=6.83$ min (major) and $T_{\mathrm{R}}=7.50 \mathrm{~min}$ (minor). 
$[\alpha]^{25} \mathrm{D}=+55.0\left(c=2.0, \mathrm{CHCl}_{3}\right)$.

(R, E)-1-(diphenylamino)-3-methylhex-3-en-2-ol (5h)<smiles>C/C=C(\C)[C@H](O)CN(c1ccccc1)c1ccccc1</smiles>

Purification by column chromatography (gradient elution with 2-9\% ethyl acetate-hexane) afforded the desired product (49 $\mathrm{mg}, 70 \%)$ as colorless oil.

${ }^{1} \mathbf{H}$ NMR $\left(500 \mathrm{MHz}, \mathrm{CDCl}_{3}\right) \delta 7.28-7.24(\mathrm{~m}, 4 \mathrm{H}), 7.06-7.04(\mathrm{~m}, 4 \mathrm{H}), 6.98-6.95(\mathrm{~m}, 2 \mathrm{H}), 5.46(\mathrm{t}, \mathrm{J}=7.0 \mathrm{~Hz}, 1 \mathrm{H}), 4.32$ $(\mathrm{dd}, \mathrm{J}=8.6,4.2 \mathrm{~Hz}, 1 \mathrm{H}), 3.85(\mathrm{dd}, \mathrm{J}=14.8,4.2 \mathrm{~Hz}, 1 \mathrm{H}), 3.73(\mathrm{dd}, \mathrm{J}=14.9,8.7 \mathrm{~Hz}, 1 \mathrm{H}), 2.06-1.98(\mathrm{~m}, 3 \mathrm{H}), 1.61(\mathrm{~s}, 3 \mathrm{H}), 0.95(\mathrm{t}$, $\mathrm{J}=7.5 \mathrm{~Hz}, 3 \mathrm{H})$

${ }^{13} \mathrm{C}$ NMR $\left(125 \mathrm{MHz}, \mathrm{CDCl}_{3}\right) \delta 148.5,134.1,129.9,129.4,121.8,121.5,74.5,57.6,21.0,14.0,11.8$

IR $v_{\max } 3564,3062,3023,2963,2930,1669,1589,1496,1458,1362,1270,1246,1095,1029,954,860 \mathrm{~cm}^{-1}$.

LRMS (ESI): Calcd. for $\mathrm{C}_{19} \mathrm{H}_{23} \mathrm{NNaO}: \mathrm{m} / \mathrm{z} 304.17\left([\mathrm{M}+\mathrm{Na}]^{+}\right)$, found: $\mathrm{m} / \mathrm{z}(\%)=304.16\left([\mathrm{M}+\mathrm{Na}]^{+}\right)$

HPLC: $96 \%$ ee, IA-3, 2-propanol: $n$-Hexane $=5: 95$, flow: $1.0 \mathrm{~mL} / \mathrm{min}, 220 \mathrm{~nm}, T_{\mathrm{R}}=7.62 \mathrm{~min}$ (major) and $T_{\mathrm{R}}=8.35 \mathrm{~min}$ (minor).

$[\alpha]^{25} \mathrm{D}=+54.6\left(c=1.0, \mathrm{CHCl}_{3}\right)$.

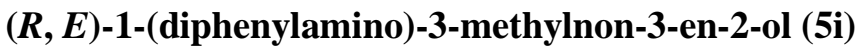<smiles>C/C(=C\[PbH])[C@@H](O)CN(c1ccccc1)c1ccccc1</smiles>

Purification by column chromatography (gradient elution with 2-8\% ethyl acetate-hexane) afforded the desired product (55 $\mathrm{mg}, 68 \%)$ as colorless oil.

${ }^{1} \mathrm{H}$ NMR (500MHz, $\left.\mathrm{CDCl}_{3}\right) \delta 7.28-7.25(\mathrm{~m}, 4 \mathrm{H}), 7.06-7.04(\mathrm{~m}, 4 \mathrm{H}), 6.98-6.95(\mathrm{~m}, 2 \mathrm{H}), 5.48(\mathrm{t}, \mathrm{J}=7.1 \mathrm{~Hz}, 1 \mathrm{H}), 4.33$ $(\mathrm{dd}, \mathrm{J}=8.6,4.0 \mathrm{~Hz}, 1 \mathrm{H}), 3.86(\mathrm{dd}, \mathrm{J}=14.8,4.1 \mathrm{~Hz}, 1 \mathrm{H}), 3.74(\mathrm{dd}, \mathrm{J}=14.8,8.8 \mathrm{~Hz}, 1 \mathrm{H}), 2.04-1.96(\mathrm{~m}, 3 \mathrm{H}), 1.62(\mathrm{~s}, 3 \mathrm{H}), 1.35-$ $1.25(\mathrm{~m}, 6 \mathrm{H}), 0.88(\mathrm{t}, \mathrm{J}=7.0 \mathrm{~Hz}, 3 \mathrm{H})$

${ }^{13} \mathbf{C ~ N M R}\left(125 \mathrm{MHz}, \mathrm{CDCl}_{3}\right) \delta 148.5,134.5,129.4,128.5,121.9,121.6,74.6,57.8,31.7,29.2,27.7,22.7,14.2,12.0$ IR $v_{\max } 3439,3024,2957,2927,2856,1669,1589,1496,1460,1362,1247,1216,1096,1028,862,838 \mathrm{~cm}^{-1}$.

LRMS (ESI): Calcd. for $\mathrm{C}_{22} \mathrm{H}_{29} \mathrm{NNaO}: \mathrm{m} / \mathrm{z} 346.21\left([\mathrm{M}+\mathrm{Na}]^{+}\right)$, found: $\mathrm{m} / \mathrm{z}(\%)=346.21\left([\mathrm{M}+\mathrm{Na}]^{+}\right)$

HPLC: $94 \%$ ee, ID, 2-propanol: $n$-Hexane=1:99, flow: 1.0mL/min, 220nm, $T_{\mathrm{R}}=10.21 \mathrm{~min}$ (major) and $T_{\mathrm{R}}=10.68 \mathrm{~min}$ (minor).

$[\alpha]^{25} \mathrm{D}=+45.0\left(c=0.50, \mathrm{CHCl}_{3}\right)$.

\section{(R)-1-(diphenylamino)-3,4-dimethylpent-3-en-2-ol (5j)}<smiles>CC(C)=C(C)[C@H](O)CN(c1ccccc1)c1ccccc1</smiles>

Purification by column chromatography (gradient elution with 2-9\% ethyl acetate-hexane) afforded the desired product (49 $\mathrm{mg}, 70 \%$ ) as colorless oil. 
${ }^{1} \mathbf{H}$ NMR $\left(500 \mathrm{MHz}, \mathrm{CDCl}_{3}\right) \delta 7.26-7.25(\mathrm{~m}, 4 \mathrm{H}), 7.07-7.05(\mathrm{~m}, 4 \mathrm{H}), 6.97-6.94(\mathrm{~m}, 2 \mathrm{H}), 4.94(\mathrm{dd}, \mathrm{J}=7.7,5.6 \mathrm{~Hz}, 1 \mathrm{H})$, $3.76(\mathrm{~d}, \mathrm{~J}=7.8 \mathrm{~Hz}, 1 \mathrm{H}), 3.75(\mathrm{~d}, \mathrm{~J}=5.5 \mathrm{~Hz}, 1 \mathrm{H}), 1.93$ (br s, 1H), $1.64(\mathrm{~s}, 3 \mathrm{H}), 1.64(\mathrm{~s}, 3 \mathrm{H}), 1.63(\mathrm{~s}, 3 \mathrm{H})$

${ }^{13} \mathbf{C ~ N M R}\left(125 \mathrm{MHz}, \mathrm{CDCl}_{3}\right) \delta 148.5,129.4,129.2,127.2,121.8,121.4,68.3,56.8,21.3,20.1,12.2$

IR $v_{\max } 3416,3055,3008,2944,2881,1664,1859,1476,1449,1366,1272,1246,1157,1055,961,871 \mathrm{~cm}^{-1}$.

LRMS (ESI): Calcd. for $\mathrm{C}_{19} \mathrm{H}_{23} \mathrm{NNaO}: \mathrm{m} / \mathrm{z} 304.17\left([\mathrm{M}+\mathrm{Na}]^{+}\right)$, found: $\mathrm{m} / \mathrm{z}(\%)=304.17\left([\mathrm{M}+\mathrm{Na}]^{+}\right)$

HPLC: $91 \%$ ee, ID, 2-propanol: $n$-Hexane=5:95, flow: 1.0mL/min, 220nm, $T_{\mathrm{R}}=6.53$ min (major) and $T_{\mathrm{R}}=7.03 \mathrm{~min}$ (minor).

$[\alpha]^{25}=+73.8\left(c=1.0, \mathrm{CHCl}_{3}\right)$.

(R, E)-1-(diphenylamino)-3-ethylideneheptan-2-ol (5k)<smiles>[10B]=[10B]/C(=C\C)[C@@H](O)CN(c1ccccc1)c1ccccc1</smiles>

Purification by column chromatography (gradient elution with 2-9\% ethyl acetate-hexane) afforded the desired product (52 $\mathrm{mg}, 67 \%)$ as orange oil.

${ }^{1}$ H NMR $\left(500 \mathrm{MHz}, \mathrm{CDCl}_{3}\right) \delta 7.28-7.25(\mathrm{~m}, 4 \mathrm{H}), 7.06(\mathrm{dd}, \mathrm{J}=8.6,0.9 \mathrm{~Hz}, 4 \mathrm{H}), 6.98-6.95(\mathrm{~m}, 2 \mathrm{H}), 5.61(\mathrm{q}, \mathrm{J}=6.8 \mathrm{~Hz}, 1 \mathrm{H})$, $4.34(\mathrm{dd}, \mathrm{J}=9.2,2.3 \mathrm{~Hz}, 1 \mathrm{H}), 3.88(\mathrm{dd}, \mathrm{J}=14.8,3.2 \mathrm{~Hz}, 1 \mathrm{H}), 3.68(\mathrm{dd}, \mathrm{J}=14.8,9.3 \mathrm{~Hz}, 1 \mathrm{H}), 2.13-2.09$ (m, $1 \mathrm{H}), 2.04$ (br s, $1 \mathrm{H})$, $2.02-1.97(\mathrm{~m}, 1 \mathrm{H}), 1.63(\mathrm{~d}, \mathrm{~J}=6.8 \mathrm{~Hz}, 3 \mathrm{H}), 1.37-1.32(\mathrm{~m}, 4 \mathrm{H}), 0.90(\mathrm{t}, \mathrm{J}=7.0 \mathrm{~Hz}, 3 \mathrm{H})$

${ }^{13}$ C NMR $\left(125 \mathrm{MHz}, \mathrm{CDCl}_{3}\right) \delta 148.6,140.5,129.5,122.0,121.9,121.6,73.3,58.7,31.8,27.5,23.2,14.1,13.2$

IR $v_{\max } 3567,3061,3021,2957,2932,1684,1590,1496,1363,1298,1246,1156,1094,1029,888,850 \mathrm{~cm}^{-1}$.

LRMS (ESI): Calcd. for $\mathrm{C}_{21} \mathrm{H}_{27} \mathrm{NNaO}: \mathrm{m} / \mathrm{z} 332.20\left([\mathrm{M}+\mathrm{Na}]^{+}\right)$, found: $\mathrm{m} / \mathrm{z}(\%)=332.20\left([\mathrm{M}+\mathrm{Na}]^{+}\right)$

HPLC: $98 \%$ ee, ID, 2-propanol: $n$-Hexane=5:95, flow: 1.0mL/min, 220nm, $T_{\mathrm{R}}=6.02 \mathrm{~min}$ (major) and $T_{\mathrm{R}}=6.42 \mathrm{~min}$ (minor).

$[\alpha]^{25} \mathrm{D}=+41.5\left(c=1.0, \mathrm{CHCl}_{3}\right)$

(R, E)-3-benzyl-1-(diphenylamino)pent-3-en-2-ol (5l)<smiles>C/C=C(/Cc1ccccc1)[C@@H](O)CN(c1ccccc1)c1ccccc1</smiles>

Purification by column chromatography (gradient elution with 2-5\% ethyl acetate-hexane) afforded the desired product (52 $\mathrm{mg}, 61 \%)$ as yellow oil.

${ }^{1} \mathbf{H}$ NMR $\left(500 \mathrm{MHz}, \mathrm{CDCl}_{3}\right) \delta 7.28-7.16(\mathrm{~m}, 9 \mathrm{H}), 6.95-6.92(\mathrm{~m}, 6 \mathrm{H}), 5.87(\mathrm{q}, \mathrm{J}=6.8 \mathrm{~Hz}, 1 \mathrm{H}), 4.34(\mathrm{dd}, \mathrm{J}=9.5,2.6 \mathrm{~Hz}, 1 \mathrm{H})$, 3.69 (dd, J=14.9, 3.2Hz, 1H), 3.54 (dd, J=15.0, 9.4Hz, 1H), 3.53 (d, J=15.0Hz, 1 H), 3.46 (d, J=15.3Hz, $1 \mathrm{H}), 2.06$ (br s, $1 \mathrm{H})$, $1.75(\mathrm{~d}, \mathrm{~J}=6.8 \mathrm{~Hz}, 3 \mathrm{H})$

${ }^{13} \mathbf{C}$ NMR $\left(125 \mathrm{MHz}, \mathrm{CDCl}_{3}\right) \delta 148.4,140.0,138.7,129.4,128.6(2 \mathrm{C}), 126.2,123.5,121.8,121.5,72.7,58.8,33.5,13.7$

IR $v_{\max } 3561,3060,3026,2927,1643,1588,1495,1453,1363,1265,1247,1208,1093,1029,848,739 \mathrm{~cm}^{-1}$.

HRMS (ESI, positive mode): Calcd. for $\mathrm{C}_{24} \mathrm{H}_{25} \mathrm{NONa}: \mathrm{m} / \mathrm{z} 366.1828\left([\mathrm{M}+\mathrm{Na}]^{+}\right)$, found: m/z $366.1831\left([\mathrm{M}+\mathrm{Na}]^{+}\right)$ HPLC: $96 \%$ ee, IC, 2-propanol: $n$-Hexane=1:99, flow: $1.0 \mathrm{~mL} / \mathrm{min}, 220 \mathrm{~nm}, T_{\mathrm{R}}=8.26 \mathrm{~min}$ (major) and $T_{\mathrm{R}}=9.54 \mathrm{~min}$ (minor).

$[\alpha]^{25} \mathrm{D}=+39.0\left(c=1.0, \mathrm{CHCl}_{3}\right)$. 
<smiles>OC(Cn1c2ccccc2c2ccccc21)C1=CCCCC1</smiles>

Purification by column chromatography (gradient elution with 2-9\% ethyl acetate-hexane) afforded the desired product (69 mg, $95 \%)$ as white solid.

${ }^{1} \mathbf{H}$ NMR $\left(500 \mathrm{MHz}, \mathrm{CDCl}_{3}\right) \delta 8.06(\mathrm{~d}, \mathrm{~J}=7.8 \mathrm{~Hz}, 2 \mathrm{H}), 7.43(\mathrm{~d}, \mathrm{~J}=3.7 \mathrm{~Hz}, 4 \mathrm{H}), 7.23-7.18(\mathrm{~m}, 2 \mathrm{H}), 5.73(\mathrm{br} \mathrm{s}, 1 \mathrm{H}), 4.43(\mathrm{dd}$, $\mathrm{J}=7.8,3.9 \mathrm{~Hz}, 1 \mathrm{H}), 4.34(\mathrm{dd}, \mathrm{J}=14.8,8.0 \mathrm{~Hz}, 1 \mathrm{H}), 4.29(\mathrm{dd}, \mathrm{J}=14.8,4.0 \mathrm{~Hz}, 1 \mathrm{H}), 2.15-1.93(\mathrm{~m}, 4 \mathrm{H}), 1.69$ (br s, $1 \mathrm{H}), 1.67-$ $1.53(\mathrm{~m}, 4 \mathrm{H})$

${ }^{13}$ C NMR (125MHz, $\left.\mathrm{CDCl}_{3}\right) \delta 140.8,137.4,125.7,124.4,123.0,120.3,119.2,109.2,75.0,48.7,25.0,24.6,22.6,22.4$

IR $v_{\max } 3552,3051,3022,2928,1627,1597,1484,1459,1380,1348,1230,1153,1090,1023,920,843 \mathrm{~cm}^{-1}$.

HRMS (ESI, positive mode): Calcd. for $\mathrm{C}_{20} \mathrm{H}_{21} \mathrm{NONa} \mathrm{m} / \mathrm{z} 314.1515\left([\mathrm{M}+\mathrm{Na}]^{+}\right)$, found: $\mathrm{m} / \mathrm{z} 314.1517\left([\mathrm{M}+\mathrm{Na}]^{+}\right)$

HPLC: $91 \%$ ee, ID, 2-propanol: $n$-Hexane $=5: 95$, flow: $1.0 \mathrm{~mL} / \mathrm{min}, 220 \mathrm{~nm}, T_{\mathrm{R}}=9.80 \mathrm{~min}$ (major) and $T_{\mathrm{R}}=10.49 \mathrm{~min}$ (minor).

$[\alpha]^{25}=+5.5\left(c=1.0, \mathrm{CHCl}_{3}\right)$.

$(R, E)-1-(9 H$-carbazol-9-yl)-3-methyl-4-phenylbut-3-en-2-ol (5n)<smiles>CC(=Cc1ccccc1)C(O)Cn1c2ccccc2c2ccccc21</smiles>

Purification by column chromatography (gradient elution with 3-9\% ethyl acetate-hexane) afforded the desired product (76 $\mathrm{mg}, 93 \%)$ as yellow oil.

${ }^{1} \mathrm{H}$ NMR $\left(500 \mathrm{MHz}, \mathrm{CDCl}_{3}\right) \delta 8.07(\mathrm{~d}, \mathrm{~J}=7.8 \mathrm{~Hz}, 2 \mathrm{H}), 7.46-7.44(\mathrm{~m}, 4 \mathrm{H}), 7.31(\mathrm{dd}, \mathrm{J}=7.5,7.5 \mathrm{~Hz}, 2 \mathrm{H}), 7.24-7.21(\mathrm{~m}, 3 \mathrm{H})$, $7.16(\mathrm{~d}, \mathrm{~J}=7.5 \mathrm{~Hz}, 2 \mathrm{H}), 6.54(\mathrm{br} \mathrm{s}, 1 \mathrm{H}), 4.61(\mathrm{dd}, \mathrm{J}=7.1,5.0 \mathrm{~Hz}, 1 \mathrm{H}), 4.41(\mathrm{dd}, \mathrm{J}=14.9,7.3 \mathrm{~Hz}, 1 \mathrm{H}), 4.37$ (dd, J=14.8, 4.8Hz, $1 \mathrm{H}), 1.97(\mathrm{~s}, 3 \mathrm{H}), 1.84(\mathrm{br} \mathrm{s}, 1 \mathrm{H})$

${ }^{13}$ C NMR $\left(125 \mathrm{MHz}, \mathrm{CDCl}_{3}\right) \delta 140.8,137.4,137.1,129.0,128.2,127.0,126.8,125.9,123.1,120.4,119.3,109.1,76.4,48.6$, 14.3

IR $v_{\max } 3556,3053,3022,2976,1627,1598,1485,1459,1349,1251,1154,1122,1051,1022,920,868 \mathrm{~cm}^{-1}$.

HRMS (ESI, positive mode): Calcd. for $\mathrm{C}_{23} \mathrm{H}_{21} \mathrm{NONa}$ : m/z $350.1515\left([\mathrm{M}+\mathrm{Na}]^{+}\right)$, found: $\mathrm{m} / \mathrm{z} 350.1516\left([\mathrm{M}+\mathrm{Na}]^{+}\right)$

HPLC: $85 \%$ ee, IA, 2-propanol: $n$-Hexane=1:9, flow: $1.0 \mathrm{~mL} / \mathrm{min}, 220 \mathrm{~nm}, T_{\mathrm{R}}=8.66 \mathrm{~min}$ (minor) and $T_{\mathrm{R}}=9.96 \mathrm{~min}$ (major). $[\alpha]^{25} \mathrm{D}=+31.9\left(c=1.0, \mathrm{CHCl}_{3}\right)$.

$(R, E)-1-(9 H$-carbazol-9-yl)-4-(4-methoxyphenyl)-3-methylbut-3-en-2-ol (5o) 
<smiles>COc1ccc(/C=C(\C)[C@H](O)Cn2c3ccccc3c3ccccc32)cc1</smiles>

Purification by column chromatography (gradient elution with 3-11\% ethyl acetate-hexane) afforded the desired product (50 mg, 56\%) as colorless oil.

${ }^{1} \mathbf{H}$ NMR $\left(500 \mathrm{MHz}, \mathrm{CDCl}_{3}\right) \delta 8.09(\mathrm{~d}, \mathrm{~J}=7.7 \mathrm{~Hz}, 2 \mathrm{H}), 7.50-7.44(\mathrm{~m}, 4 \mathrm{H}), 7.24-7.22(\mathrm{~m}, 2 \mathrm{H}), 7.13(\mathrm{~d}, \mathrm{~J}=8.6 \mathrm{~Hz}, 2 \mathrm{H}), 6.86$ - 6.85 (m, 2H), 6.51 (br s, 1H), 4.66 (dd, J=7.6, 4.4Hz, 1H), 4.45 (dd, J=15.0, 7.9Hz, 1H), 4.41 (dd, J=15.0, 4.5Hz, 1H), 3.80 (s, 3H), $1.99(\mathrm{~d}, \mathrm{~J}=1.2 \mathrm{~Hz}, 3 \mathrm{H}), 1.85$ (br s, 1H)

${ }^{13} \mathbf{C}$ NMR $\left(125 \mathrm{MHz}, \mathrm{CDCl}_{3}\right) \delta 158.5,140.9,135.8,130.3,129.7,126.6,125.9,123.1,120.5,119.3,113.7,109.2,76.7,55.4$, $48.8,14.4$

IR $v_{\max } 3549,3052,3012,2957,2855,1606,1510,1484,1459,1349,1299,1216,1178,1035,951,833 \mathrm{~cm}^{-1}$.

LRMS (ESI): Calcd. for $\mathrm{C}_{24} \mathrm{H}_{23} \mathrm{NNaO}_{2}: \mathrm{m} / \mathrm{z} 380.16\left([\mathrm{M}+\mathrm{Na}]^{+}\right)$, found: $\mathrm{m} / \mathrm{z}(\%)=380.16\left([\mathrm{M}+\mathrm{Na}]^{+}\right)$

HPLC: $80 \%$ ee, ID, 2-propanol: $n$-Hexane=1:9, flow: $1.0 \mathrm{~mL} / \mathrm{min}, 220 \mathrm{~nm}, T_{\mathrm{R}}=10.46 \mathrm{~min}$ (major) and $T_{\mathrm{R}}=11.48 \mathrm{~min}$ (minor).

$[\alpha]^{25} \mathrm{D}=+33.2\left(c=1.0, \mathrm{CHCl}_{3}\right)$

\section{(R, E)-4-(4-bromophenyl)-1-(9H-carbazol-9-yl)-3-methylbut-3-en-2-ol (5p)}<smiles>C/C(=C\c1ccc(Br)cc1)[C@@H](O)Cn1c2ccccc2c2ccccc21</smiles>

Purification by column chromatography (gradient elution with 3-11\% ethyl acetate-hexane) afforded the desired product (85 $\mathrm{mg}, 84 \%)$ as white solid.

${ }^{1}$ H NMR $\left(500 \mathrm{MHz}, \mathrm{CDCl}_{3}\right) \delta 8.07(\mathrm{~d}, \mathrm{~J}=7.8 \mathrm{~Hz}, 2 \mathrm{H}), 7.44(\mathrm{~d}, \mathrm{~J}=3.7 \mathrm{~Hz}, 4 \mathrm{H}), 7.41(\mathrm{~d}, \mathrm{~J}=8.4 \mathrm{~Hz}, 2 \mathrm{H}), 7.24-7.21(\mathrm{~m}, 2 \mathrm{H})$, 6.97 (d, J=8.3Hz, 2H), 6.40 (br s, 1H), 4.60 (dd, J=6.2, 6.0Hz, 1H), 4.40 (dd, J=15.1, 7.0Hz, 1H), 4.37 (dd, J=15.0, 5.4Hz, $1 \mathrm{H}), 1.92(\mathrm{~d}, \mathrm{~J}=1.0 \mathrm{~Hz}, 3 \mathrm{H}), 1.87(\mathrm{br} \mathrm{s}, 1 \mathrm{H})$

${ }^{13}$ C NMR $\left(125 \mathrm{MHz}, \mathrm{CDCl}_{3}\right) \delta 140.8,138.2,135.9,131.3,130.6,125.9,125.8,123.1,120.6,120.4,119.4,109.1,76.1,48.4$, 14.4

IR $v_{\max } 3550,3051,3021,2934,1627,1597,1485,1459,1348,1251,1154,1122,1072,949,852,648 \mathrm{~cm}^{-1}$.

LRMS (ESI): Calcd. for $\mathrm{C}_{23} \mathrm{H}_{20} \mathrm{BrNNaO}: \mathrm{m} / \mathrm{z} 428.06\left([\mathrm{M}+\mathrm{Na}]^{+}\right)$, found: $\mathrm{m} / \mathrm{z}(\%)=428.06\left([\mathrm{M}+\mathrm{Na}]^{+}, 100\right), 430.06(98)$

HPLC: $87 \%$ ee, OZ-H, 2-propanol: $n$-Hexane=1:9, flow: $1.0 \mathrm{~mL} / \mathrm{min}, 220 \mathrm{~nm}, T_{\mathrm{R}}=4.91 \mathrm{~min}(\operatorname{minor})$ and $T_{\mathrm{R}}=5.87 \mathrm{~min}$ (major).

$[\alpha]^{25} \mathrm{D}=+34.2\left(c=2.0, \mathrm{CHCl}_{3}\right)$.

(R)-1-cyclopropyl-2-(diphenylamino)ethan-1-ol (5q) 
<smiles>OC(CNc1ccccc1)C1CC1</smiles>

Purification by column chromatography (gradient elution with 2-6\% ethyl acetate-hexane) afforded the desired product (60 $\mathrm{mg}, 95 \%)$ as white solid.

${ }^{1} \mathbf{H}$ NMR $\left(500 \mathrm{MHz}, \mathrm{CDCl}_{3}\right) \delta 7.27-7.23(\mathrm{~m}, 4 \mathrm{H}), 7.06(\mathrm{~d}, \mathrm{~J}=7.5 \mathrm{~Hz}, 4 \mathrm{H}), 6.97-6.94$ (m, 2H), 3.99 (dd, J=14.9, 3.8Hz, 1H), 3.80 (dd, J=14.9, 8.6Hz, 1H), 3.22 (ddd, J=8.5, 8.5, 3.7Hz, 1H), 2.08 (br s, 1H), $0.92-0.87$ (m, $1 \mathrm{H}), 0.54-0.50$ (m, $2 \mathrm{H}), 0.34-0.32(\mathrm{~m}, 1 \mathrm{H}), 0.26-0.23(\mathrm{~m}, 1 \mathrm{H})$

${ }^{13}$ C NMR $\left(125 \mathrm{MHz}, \mathrm{CDCl}_{3}\right) \delta 148.6,129.4,121.8,121.5,73.6,58.8,15.2,2.6,2.4$

IR $v_{\max } 3666,2967,2940,2923,2866,1516,1477,1455,1346,1322,1216,1055,1032,1012 \mathrm{~cm}^{-1}$.

HRMS (ESI, positive mode): Calcd. for $\mathrm{C}_{17} \mathrm{H}_{19} \mathrm{NONa}: \mathrm{m} / \mathrm{z} 276.1361\left([\mathrm{M}+\mathrm{Na}]^{+}\right)$, found: m/z $276.1359\left([\mathrm{M}+\mathrm{Na}]^{+}\right)$

HPLC: $82 \%$ ee, ID, 2-propanol: $n$-Hexane=5:95, flow: 1.0mL/min, 220nm, $T_{\mathrm{R}}=8.12$ min (major) and $T_{\mathrm{R}}=8.68 \mathrm{~min}$ (minor).

$[\alpha]^{25}=+34.9\left(c=1.0, \mathrm{CHCl}_{3}\right)$.

\section{(R)-1-(diphenylamino)butan-2-ol (5r)}<smiles>CC[C@H](O)C[PbH2]</smiles>

Purification by column chromatography (gradient elution with 4-11\% ethyl acetate-hexane) afforded the desired product (25 $\mathrm{mg}, 41 \%)$ as colorless oil.

${ }^{1}$ H NMR $\left(500 \mathrm{MHz}, \mathrm{CDCl}_{3}\right) \delta 7.28-7.24(\mathrm{~m}, 4 \mathrm{H}), 7.06-7.03(\mathrm{~m}, 4 \mathrm{H}), 6.99-6.95(\mathrm{~m}, 2 \mathrm{H}), 3.86-3.82(\mathrm{~m}, 2 \mathrm{H}), 3.61(\mathrm{dd}$, $\mathrm{J}=15.3,9.8 \mathrm{~Hz}, 1 \mathrm{H}), 2.12$ (br s, $1 \mathrm{H}), 1.57-1.47(\mathrm{~m}, 2 \mathrm{H}), 0.99(\mathrm{t}, \mathrm{J}=7.5 \mathrm{~Hz}, 3 \mathrm{H})$

${ }^{13}$ C NMR (125MHz, $\left.\mathrm{CDCl}_{3}\right) \delta 148.7,129.5,122.0,121.6,70.3,59.1,27.4,10.1$

IR $v_{\max } 3664,3009,2965,2937,2903,1590,1496,1457,1359,1246,1216,1058,1007,992,871 \mathrm{~cm}^{-1}$.

LRMS (ESI): Calcd. for $\mathrm{C}_{16} \mathrm{H}_{19} \mathrm{NNaO}: \mathrm{m} / \mathrm{z} 264.14\left([\mathrm{M}+\mathrm{Na}]^{+}\right)$, found: $\mathrm{m} / \mathrm{z}(\%)=264.14\left([\mathrm{M}+\mathrm{Na}]^{+}\right)$

HPLC: $52 \%$ ee, IA-3, 2-propanol: $n$-Hexane=5:95, flow: $1.0 \mathrm{~mL} / \mathrm{min}, 220 \mathrm{~nm}, T_{\mathrm{R}}=9.45 \min (\operatorname{minor})$ and $T_{\mathrm{R}}=10.41 \mathrm{~min}$ (major).

$[\alpha]^{25}=-7.1\left(c=1.0, \mathrm{CHCl}_{3}\right)$.

\section{(R)-2-(diphenylamino)-1-phenylethanol (6a)}<smiles>O[C@H](CN(c1ccccc1)c1ccccc1)c1ccccc1</smiles>

Purification by column chromatography (gradient elution with 2-9\% ethyl acetate-hexane) afforded the desired product (64 $\mathrm{mg}, 88 \%)$ as colorless oil.

${ }^{1}$ H NMR (500MHz, $\left.\mathrm{CDCl}_{3}\right) \delta 7.39$ - $7.35(\mathrm{~m}, 4 \mathrm{H}), 7.29-7.26(\mathrm{~m}, 5 \mathrm{H}), 7.07-7.05(\mathrm{~m}, 4 \mathrm{H}), 7.00-6.97(\mathrm{~m}, 2 \mathrm{H}), 4.98(\mathrm{dd}$, $\mathrm{J}=9.1,3.9 \mathrm{~Hz}, 1 \mathrm{H}), 3.96(\mathrm{dd}, \mathrm{J}=15.0,4.0 \mathrm{~Hz}, 1 \mathrm{H}), 3.88(\mathrm{dd}, \mathrm{J}=15.0,9.2 \mathrm{~Hz}, 1 \mathrm{H}), 2.48(\mathrm{~s}, 1 \mathrm{H})$

${ }^{13} \mathbf{C}$ NMR $\left(125 \mathrm{MHz}, \mathrm{CDCl}_{3}\right) \delta 148.4,141.8,129.5,128.7,128.0,126.1,122.1,121.6,71.5,60.9$

IR $v_{\max } 3554,3008,2973,2940,2866,1588,1495,1454,1361,1246,1217,1056,1033,915,898,867 \mathrm{~cm}^{-1}$.

HRMS (ESI, positive mode): Calcd. for $\mathrm{C}_{20} \mathrm{H}_{19} \mathrm{NONa}: \mathrm{m} / \mathrm{z} 312.1359\left([\mathrm{M}+\mathrm{Na}]^{+}\right)$, found: $\mathrm{m} / \mathrm{z} 312.1362\left([\mathrm{M}+\mathrm{Na}]^{+}\right)$ 
HPLC: $92 \%$ ee, ID, 2-propanol: $n$-Hexane=5:95, flow: 1.0mL/min, 220nm, $T_{\mathrm{R}}=7.90 \mathrm{~min}$ (major) and $T_{\mathrm{R}}=8.45 \mathrm{~min}$ (minor).

$[\alpha]^{25} \mathrm{D}=+42.7\left(c=1.0, \mathrm{CHCl}_{3}\right)$.

(R)-2-(diphenylamino)-1-(4-methoxyphenyl)ethanol (6b)<smiles>COc1ccc([C@H](O)CN(c2ccccc2)c2ccccc2)cc1</smiles>

Purification by column chromatography (gradient elution with $2-14 \%$ ethyl acetate-hexane) afforded the desired product (65 $\mathrm{mg}, 81 \%)$ as colorless oil.

${ }^{1}$ H NMR $\left(500 \mathrm{MHz}, \mathrm{CDCl}_{3}\right) \delta 7.28-7.24(\mathrm{~m}, 6 \mathrm{H}), 7.04(\mathrm{~d}, \mathrm{~J}=7.7 \mathrm{~Hz}, 4 \mathrm{H}), 6.97(\mathrm{dd}, \mathrm{J}=7.3,7.3 \mathrm{~Hz}, 2 \mathrm{H}), 6.87(\mathrm{~d}, \mathrm{~J}=8.7 \mathrm{~Hz}$, 2H), 4.91 (ddd, J=8.6, 4.4, 1.9Hz, 1H), 3.91 (dd, J=14.9, 4.4Hz, 1H), $3.86(\mathrm{dd}, \mathrm{J}=14.9,8.7 \mathrm{~Hz}, 1 \mathrm{H}), 3.78$ (s, $3 \mathrm{H}), 2.47$ (d, $\mathrm{J}=1.9 \mathrm{~Hz}, 1 \mathrm{H})$

${ }^{13}$ C NMR $\left(125 \mathrm{MHz}, \mathrm{CDCl}_{3}\right) \delta 159.4,148.4,133.9,129.5,127.4,122.0,121.6,114.0,71.0,60.8,55.4$ IR $v_{\max } 3547,3060,3022,2957,2929,1588,1513,1495,1459,1362,1246,1175,1095,1070,972,832 \mathrm{~cm}^{-1}$.

LRMS (ESI): Calcd. for $\mathrm{C}_{21} \mathrm{H}_{21} \mathrm{KNO}_{2}: \mathrm{m} / \mathrm{z} 358.12\left([\mathrm{M}+\mathrm{K}]^{+}\right)$, found: $\mathrm{m} / \mathrm{z}(\%)=358.12\left([\mathrm{M}+\mathrm{K}]^{+}\right)$

HPLC: $90 \%$ ee, ID, 2-propanol: $n$-Hexane=5:95, flow: $1.0 \mathrm{~mL} / \mathrm{min}, 220 \mathrm{~nm}, T_{\mathrm{R}}=13.79$ min (major) and $T_{\mathrm{R}}=15.42 \mathrm{~min}$ (minor).

$[\alpha]^{25}=+43.8\left(c=1.6, \mathrm{CHCl}_{3}\right)$.

\section{(R)-2-(diphenylamino)-1-p-tolylethanol (6c)}<smiles>Cc1ccc([C@@H](O)CN(c2ccccc2)c2ccccc2)cc1</smiles>

Purification by column chromatography (gradient elution with 14-33\% dichloromethane-hexane) afforded the desired product $(65 \mathrm{mg}, 86 \%)$ as light yellow oil.

${ }^{1}$ H NMR $\left(500 \mathrm{MHz}, \mathrm{CDCl}_{3}\right) \delta 7.29-7.25(\mathrm{~m}, 6 \mathrm{H}), 7.16(\mathrm{~d}, \mathrm{~J}=7.8 \mathrm{~Hz}, 2 \mathrm{H}), 7.07-7.05(\mathrm{~m}, 4 \mathrm{H}), 6.99-6.96(\mathrm{~m}, 2 \mathrm{H}), 4.94$ (ddd, J=9.1, 3.7, 2.0Hz, 1H), 3.93 (dd, J=14.9, 3.7Hz, 1H), 3.86 (dd, J=14.9, 9.2Hz, 1H), 2.43 (d, J=2.0Hz, 1H), 2.34 (s, 3H) ${ }^{13}$ C NMR $\left(125 \mathrm{MHz}, \mathrm{CDCl}_{3}\right) \delta 148.4,138.8,137.7,129.5,129.3,126.1,122.0,121.6,71.4,60.9,21.3$ IR $v_{\max } 3562,3056,3036,2893,2876,1588,1495,1450,1362,1297,1265,1191,1070,968,899,817 \mathrm{~cm}^{-1}$.

LRMS (ESI): Calcd. for $\mathrm{C}_{21} \mathrm{H}_{21} \mathrm{NNaO}: \mathrm{m} / \mathrm{z} 326.15\left([\mathrm{M}+\mathrm{Na}]^{+}\right)$, found: $\mathrm{m} / \mathrm{z}(\%)=326.15\left([\mathrm{M}+\mathrm{Na}]^{+}\right)$

HPLC: $90 \%$ ee, ID, 2-propanol: $n$-Hexane=5:95, flow: 1.0mL/min, 220nm, $T_{\mathrm{R}}=8.46 \mathrm{~min}$ (major) and $T_{\mathrm{R}}=9.41 \mathrm{~min}$ (minor).

$[\alpha]^{25}=+49.2\left(c=1.6, \mathrm{CHCl}_{3}\right)$

\section{(R)-1-(4-bromophenyl)-2-(diphenylamino)ethanol (6d)}<smiles>O[C@H](CN(c1ccccc1)c1ccccc1)c1ccc(Br)cc1</smiles> 
Purification by column chromatography (gradient elution with 14-33\% dichloromethane-hexane) afforded the desired product $(72 \mathrm{mg}, 78 \%$ ) as colorless oil.

${ }^{1} \mathbf{H}$ NMR $\left(500 \mathrm{MHz}, \mathrm{CDCl}_{3}\right) \delta 7.48-7.46(\mathrm{~m}, 2 \mathrm{H}), 7.29-7.24(\mathrm{~m}, 6 \mathrm{H}), 7.04(\mathrm{~d}, \mathrm{~J}=7.7 \mathrm{~Hz}, 4 \mathrm{H}), 6.99(\mathrm{dd}, \mathrm{J}=7.3,7.3 \mathrm{~Hz}, 2 \mathrm{H})$, $4.93(\mathrm{dd}, \mathrm{J}=9.2,3.9 \mathrm{~Hz}, 1 \mathrm{H}), 3.92(\mathrm{dd}, \mathrm{J}=14.9,4.0 \mathrm{~Hz}, 1 \mathrm{H}), 3.82(\mathrm{dd}, \mathrm{J}=14.9,9.2 \mathrm{~Hz}, 1 \mathrm{H}), 2.53$ (br s, 1H)

${ }^{13}$ C NMR $\left(125 \mathrm{MHz}, \mathrm{CDCl}_{3}\right) \delta 148.2,140.6,131.6,129.5,127.7,122.2,121.7,121.5,70.8,60.7$

IR $v_{\max } 3518,3061,3034,2908,2867,1589,1494,1450,1362,1271,1246,1157,1071,968,823,697 \mathrm{~cm}^{-1}$.

LRMS (ESI): Calcd. for $\mathrm{C}_{20} \mathrm{H}_{18} \mathrm{BrNNaO}: \mathrm{m} / \mathrm{z} 390.05\left([\mathrm{M}+\mathrm{Na}]^{+}\right)$, found: $\mathrm{m} / \mathrm{z}(\%)=390.04\left([\mathrm{M}+\mathrm{Na}]^{+}, 100\right), 392.05(98)$

HPLC: $86 \%$ ee, ID, 2-propanol: $n$-Hexane=5:95, flow: 1.0mL/min, 220nm, $T_{\mathrm{R}}=7.52$ min (major) and $T_{\mathrm{R}}=7.92 \mathrm{~min}$ (minor).

$[\alpha]^{25}=+46.1\left(c=1.0, \mathrm{CHCl}_{3}\right)$.

\section{(R)-1-(4-chlorophenyl)-2-(diphenylamino)ethan-1-ol (6e)}<smiles>O[C@H](CN(c1ccccc1)c1ccccc1)c1ccc(Cl)cc1</smiles>

Purification by column chromatography (gradient elution with 2-9\% ethyl acetate-hexane) afforded the desired product (72 $\mathrm{mg}, 89 \%)$ as colorless oil.

${ }^{1}$ H NMR $\left(500 \mathrm{MHz}, \mathrm{CDCl}_{3}\right) \delta 7.31(\mathrm{~s}, 4 \mathrm{H}), 7.28-7.25(\mathrm{~m}, 4 \mathrm{H}), 7.03(\mathrm{~d}, \mathrm{~J}=7.6 \mathrm{~Hz}, 4 \mathrm{H}), 6.99-6.97(\mathrm{~m}, 2 \mathrm{H}), 4.93(\mathrm{dd}, \mathrm{J}=9.1$, $4.0 \mathrm{~Hz}, 1 \mathrm{H}), 3.91(\mathrm{dd}, \mathrm{J}=14.9,4.0 \mathrm{~Hz}, 1 \mathrm{H}), 3.82(\mathrm{dd}, \mathrm{J}=14.9,9.1 \mathrm{~Hz}, 1 \mathrm{H}), 2.55$ (br s, 1H)

${ }^{13}$ C NMR $\left(125 \mathrm{MHz}, \mathrm{CDCl}_{3}\right) \delta 148.3,140.2,133.7,129.6,128.8,127.5,122.3,121.6,70.8,60.8$

IR $v_{\max } 3549,3064,2903,2864,1600,1589,1494,1363,1271,1246,1217,1091,1070,1014,899,829,668 \mathrm{~cm}^{-1}$.

LRMS (ESI): Calcd. for $\mathrm{C}_{20} \mathrm{H}_{18} \mathrm{ClNNaO}: \mathrm{m} / \mathrm{z} 346.10\left([\mathrm{M}+\mathrm{Na}]^{+}\right)$, found: $\mathrm{m} / \mathrm{z}(\%)=346.09\left([\mathrm{M}+\mathrm{Na}]^{+}, 100\right), 348.09(32)$

HPLC: $84 \%$ ee, OZ-H, 2-propanol: $n$-Hexane=1:99, flow: $1.0 \mathrm{~mL} / \mathrm{min}, 220 \mathrm{~nm}, T_{\mathrm{R}}=13.58 \mathrm{~min}(\operatorname{minor})$ and $T_{\mathrm{R}}=17.80 \mathrm{~min}$ (major).

$[\alpha]^{25}=+41.8\left(c=2.0, \mathrm{CHCl}_{3}\right)$

\section{(R)-2-(diphenylamino)-1-(m-tolyl)ethan-1-ol (6f)}<smiles>Cc1cccc([C@@H](O)CN(c2ccccc2)c2ccccc2)c1</smiles>

Purification by column chromatography (gradient elution with 2-6\% ethyl acetate-hexane) afforded the desired product (73 $\mathrm{mg}, 96 \%)$ as colorless oil.

${ }^{1}$ H NMR $\left(500 \mathrm{MHz}, \mathrm{CDCl}_{3}\right) \delta 7.30-7.26(\mathrm{~m}, 4 \mathrm{H}), 7.23(\mathrm{~d}, \mathrm{~J}=6.1 \mathrm{~Hz}, 2 \mathrm{H}), 7.19(\mathrm{~d}, \mathrm{~J}=7.6 \mathrm{~Hz}, 1 \mathrm{H}), 7.11(\mathrm{~d}, \mathrm{~J}=7.4 \mathrm{~Hz}, 1 \mathrm{H})$, $7.08(\mathrm{~d}, \mathrm{~J}=8.0 \mathrm{~Hz}, 4 \mathrm{H}), 6.99(\mathrm{dd}, \mathrm{J}=7.4,7.4 \mathrm{~Hz}, 2 \mathrm{H}), 4.96(\mathrm{dd}, \mathrm{J}=9.4,3.7 \mathrm{~Hz}, 1 \mathrm{H}), 3.96(\mathrm{dd}, \mathrm{J}=15.0,3.6 \mathrm{~Hz}, 1 \mathrm{H}), 3.87$ (dd, $\mathrm{J}=15.0,9.4 \mathrm{~Hz}, 1 \mathrm{H}), 2.45$ (br s, $1 \mathrm{H}), 2.36(\mathrm{~s}, 3 \mathrm{H})$

${ }^{13} \mathrm{C} \mathrm{NMR}\left(125 \mathrm{MHz}, \mathrm{CDCl}_{3}\right) \delta 148.5,141.6,138.4,129.5,128.8,128.6,126.8,123.2,122.1,121.6,71.6,60.9,21.6$ IR $v_{\max } 3666,3008,2980,2968,2938,2865,1589,1360,1346,1241,1217,1053,1031,1012,992,880 \mathrm{~cm}^{-1}$.

HRMS (ESI, positive mode): Calcd. for $\mathrm{C}_{21} \mathrm{H}_{21} \mathrm{NONa}$ : m/z $326.1515\left([\mathrm{M}+\mathrm{Na}]^{+}\right)$, found: m/z $326.1521\left([\mathrm{M}+\mathrm{Na}]^{+}\right)$ HPLC: $95 \%$ ee, IA-3, 2-propanol: $n$-Hexane=5:95, flow: $1.0 \mathrm{~mL} / \mathrm{min}, 220 \mathrm{~nm}, T_{\mathrm{R}}=10.45 \mathrm{~min}(\operatorname{major})$ and $T_{\mathrm{R}}=11.26 \mathrm{~min}$ (minor).

$[\alpha]^{25} \mathrm{D}=+13.5\left(c=1.0, \mathrm{CHCl}_{3}\right)$. 


\section{(R)-2-(diphenylamino)-1-o-tolylethanol (6g)}<smiles>Cc1ccccc1[C@@H](O)CN(c1ccccc1)c1ccccc1</smiles>

Purification by column chromatography (gradient elution with 14-33\% dichloromethane-hexane) afforded the desired product (48 mg, 64\%) as colorless oil.

${ }^{1} \mathbf{H}$ NMR $\left(500 \mathrm{MHz}, \mathrm{CDCl}_{3}\right) \delta 7.59(\mathrm{~d}, \mathrm{~J}=7.6 \mathrm{~Hz}, 1 \mathrm{H}), 7.27(\mathrm{t}, \mathrm{J}=7.9 \mathrm{~Hz}, 4 \mathrm{H}), 7.23(\mathrm{~d}, \mathrm{~J}=8.3 \mathrm{~Hz}, 1 \mathrm{H}), 7.18(\mathrm{t}, \mathrm{J}=7.3 \mathrm{~Hz}, 1 \mathrm{H})$, $7.11(\mathrm{~d}, \mathrm{~J}=7.6 \mathrm{~Hz}, 1 \mathrm{H}), 7.08(\mathrm{~d}, \mathrm{~J}=7.9 \mathrm{~Hz}, 4 \mathrm{H}), 6.99$ (t, J=7.3Hz, 2H), 5.17 (dd, J=9.2, 3.3Hz, 1H), 3.91 (dd, J=15.0, 3.4Hz, $1 \mathrm{H}), 3.84(\mathrm{dd}, \mathrm{J}=15.0,9.2 \mathrm{~Hz}, 1 \mathrm{H}), 2.46$ (br s, $1 \mathrm{H}), 2.23$ (s, 3H)

${ }^{13}$ C NMR $\left(125 \mathrm{MHz}, \mathrm{CDCl}_{3}\right) \delta 148.5,139.8,134.9,130.5,129.5,127.7,126.6,126.0,122.2,121.6,68.2,59.6,19.2$

IR $v_{\max } 3562,3061,3024,2987,2945,1588,1494,1460,1362,1343,1246,1119,1059,1028,945,868 \mathrm{~cm}^{-1}$.

LRMS (ESI): Calcd. for $\mathrm{C}_{21} \mathrm{H}_{21} \mathrm{KNO}: \mathrm{m} / \mathrm{z} 342.13\left([\mathrm{M}+\mathrm{K}]^{+}\right)$, found: $\mathrm{m} / \mathrm{z}(\%)=342.12\left([\mathrm{M}+\mathrm{K}]^{+}\right)$

HPLC: $89 \%$ ee, ID, 2-propanol: $n$-Hexane=5:95, flow: $1.0 \mathrm{~mL} / \mathrm{min}, 220 \mathrm{~nm}, T_{\mathrm{R}}=7.63 \mathrm{~min}$ (major) and $T_{\mathrm{R}}=8.44 \mathrm{~min}$ (minor).

$[\alpha]^{25}=+43.2\left(c=1.6, \mathrm{CHCl}_{3}\right)$.

\section{(R)-2-(diphenylamino)-1-(furan-3-yl)ethan-1-ol (6h)}<smiles>O[C@H](CN(c1ccccc1)c1ccccc1)c1ccoc1</smiles>

Purification by column chromatography (gradient elution with $2-11 \%$ ethyl acetate-hexane) afforded the desired product (40 $\mathrm{mg}, 57 \%)$ as brown oil.

${ }^{1}$ H NMR (500MHz, $\left.\mathrm{CDCl}_{3}\right) \delta 7.42-7.42(\mathrm{~m}, 1 \mathrm{H}), 7.39-7.38(\mathrm{~m}, 1 \mathrm{H}), 7.28-7.25(\mathrm{~m}, 4 \mathrm{H}), 7.05(\mathrm{~d}, \mathrm{~J}=7.5 \mathrm{~Hz}, 4 \mathrm{H}), 7.00-$ $6.97(\mathrm{~m}, 2 \mathrm{H}), 6.42$ (d, J=1.7Hz, 1H), 4.96 (dd, J=9.1, 4.0Hz, 1H), 3.99 (dd, J=14.8, 4.0Hz, 1H), 3.91 (dd, J=14.9, $9.1 \mathrm{~Hz}$ $1 \mathrm{H}), 2.40$ (br s, 1H)

${ }^{13}$ C NMR (125MHz, $\left.\mathrm{CDCl}_{3}\right) \delta$ 148.3, 143.6, 139.6, 129.5, 126.1, 122.2, 121.6, 108.6, 64.6, 59.6

IR $v_{\max } 3548,3010,2981,2937,2865,1589,1496,1362,1271,1248,1216,1159,1056,1023,889,875 \mathrm{~cm}^{-1}$.

LRMS (ESI): Calcd. for $\mathrm{C}_{18} \mathrm{H}_{17} \mathrm{NNaO}_{2}: \mathrm{m} / \mathrm{z} 302.12\left([\mathrm{M}+\mathrm{Na}]^{+}\right)$, found: $\mathrm{m} / \mathrm{z}(\%)=302.11\left([\mathrm{M}+\mathrm{Na}]^{+}\right)$

HPLC:89\% ee, IA-3, 2-propanol: $n$-Hexane=5:95, flow: $1.0 \mathrm{~mL} / \mathrm{min}, 220 \mathrm{~nm}, T_{\mathrm{R}}=14.13 \mathrm{~min}$ (major) and $T_{\mathrm{R}}=14.91 \mathrm{~min}$ (minor).

$[\alpha]^{25} \mathrm{D}=+37.6\left(c=1.0, \mathrm{CHCl}_{3}\right)$

\section{(R)-2-(diphenylamino)-1-(thiophen-3-yl)ethan-1-ol (6i)}<smiles>O[C@H](CN(c1ccccc1)c1ccccc1)c1ccsc1</smiles>

Purification by column chromatography (gradient elution with 2-6\% ethyl acetate-hexane) afforded the desired product (54 $\mathrm{mg}, 73 \%$ ) as colorless oil.

${ }^{1}$ H NMR $\left(500 \mathrm{MHz}, \mathrm{CDCl}_{3}\right) \delta 7.30-7.22(\mathrm{~m}, 6 \mathrm{H}), 7.09(\mathrm{dd}, \mathrm{J}=5.0,1.3 \mathrm{~Hz}, 1 \mathrm{H}), 7.04-7.02(\mathrm{~m}, 4 \mathrm{H}), 6.99-6.96(\mathrm{~m}, 2 \mathrm{H})$, $5.07(\mathrm{dd}, \mathrm{J}=8.9,4.2 \mathrm{~Hz}, 1 \mathrm{H}), 4.00(\mathrm{dd}, \mathrm{J}=15.0,4.2 \mathrm{~Hz}, 1 \mathrm{H}), 3.93(\mathrm{dd}, \mathrm{J}=15.0,8.9 \mathrm{~Hz}, 1 \mathrm{H}), 2.47$ (br s, 1H) 
${ }^{13}$ C NMR $\left(125 \mathrm{MHz}, \mathrm{CDCl}_{3}\right) \delta 148.3,143.1,129.5,126.4,125.7,122.1,121.5,121.5,68.0,60.0$

IR $v_{\max } 3557,2929,2896,2852,1601,1589,1497,1364,1272,1248,1219,1038,1027,855,839 \mathrm{~cm}^{-1}$.

LRMS (ESI): Calcd. for $\mathrm{C}_{18} \mathrm{H}_{17} \mathrm{NNaOS}: \mathrm{m} / \mathrm{z} 318.09\left([\mathrm{M}+\mathrm{Na}]^{+}\right)$, found: $\mathrm{m} / \mathrm{z}(\%)=318.09\left([\mathrm{M}+\mathrm{Na}]^{+}\right)$

HPLC: $89 \%$ ee, OZ-H, 2-propanol: $n$-Hexane=1:9, flow: $1.0 \mathrm{~mL} / \mathrm{min}, 220 \mathrm{~nm}, T_{\mathrm{R}}=4.87 \mathrm{~min}(\operatorname{minor})$ and $T_{\mathrm{R}}=5.47 \mathrm{~min}$ (major).

$[\alpha]^{25}=+43.7\left(c=1.0, \mathrm{CHCl}_{3}\right)$.

(R)-2-(diphenylamino)-1-(naphthalen-1-yl)ethan-1-ol (6j)<smiles>OC(CN(c1ccccc1)c1ccccc1)c1cccc2ccccc12</smiles>

Purification by column chromatography (gradient elution with 1-5\% ethyl acetate-hexane) afforded the desired product (83 mg, 98\%) as colorless oil.

${ }^{1} \mathbf{H}$ NMR (500MHz, $\left.\mathrm{CDCl}_{3}\right) \delta 7.87-7.84(\mathrm{~m}, 2 \mathrm{H}), 7.78$ (br s, 1H), 7.77 (d, J=2.8Hz, 1H), $7.49-7.41$ (m, $\left.3 \mathrm{H}\right), 7.31-7.28$ $(\mathrm{m}, 4 \mathrm{H}), 7.12(\mathrm{~d}, \mathrm{~J}=7.4 \mathrm{~Hz}, 4 \mathrm{H}), 7.02-6.99(\mathrm{~m}, 2 \mathrm{H}), 5.67$ (dd, J=9.4, 3.2Hz, 1H), $4.14(\mathrm{dd}, \mathrm{J}=15.1,3.3 \mathrm{~Hz}, 1 \mathrm{H}), 4.00(\mathrm{dd}$, $\mathrm{J}=15.1,9.4 \mathrm{~Hz}, 1 \mathrm{H}), 2.66$ (br s, $1 \mathrm{H})$

${ }^{13}$ C NMR $\left(125 \mathrm{MHz}, \mathrm{CDCl}_{3}\right) \delta 148.4,137.5,133.9,130.7,129.6,129.1,128.4,126.3,125.7,125.7,123.8,122.9,122.2$, $121.8,68.9,59.9$

IR $v_{\max } 3663,3003,2973,2954,2934,1589,1497,1362,1246,1217,1061,1031,1017,841,803 \mathrm{~cm}^{-1}$.

LRMS (ESI): Calcd. for $\mathrm{C}_{24} \mathrm{H}_{21} \mathrm{NNaO}: \mathrm{m} / \mathrm{z} 362.15\left([\mathrm{M}+\mathrm{Na}]^{+}\right)$, found: $\mathrm{m} / \mathrm{z}(\%)=362.15\left([\mathrm{M}+\mathrm{Na}]^{+}\right)$

HPLC: 84\% ee, IC, 2-propanol: $n$-Hexane=1:99, flow: $1.0 \mathrm{~mL} / \mathrm{min}, 220 \mathrm{~nm}, T_{\mathrm{R}}=13.22 \mathrm{~min}$ (major) and $T_{\mathrm{R}}=20.96 \mathrm{~min}$ (minor).

$[\alpha]^{25} \mathrm{D}=+29.3\left(c=1.0, \mathrm{CHCl}_{3}\right)$.

\section{(R)-2-(bis(4-methoxyphenyl)amino)-1-phenylethan-1-ol (6k)}<smiles>COc1ccc(N(C[C@H](O)c2ccccc2)c2ccc(OC)cc2)cc1</smiles>

Purification by column chromatography (gradient elution with $2-14 \%$ ethyl acetate-hexane) on activated silica gel (2.0 \% $v / v \mathrm{Et}_{3} \mathrm{~N}$ in $n$-hexane) afforded the desired product $(71 \mathrm{mg}, 81 \%)$ as colorless oil.

${ }^{1} \mathbf{H}$ NMR $\left(500 \mathrm{MHz}, \mathrm{CDCl}_{3}\right) \delta 7.39-7.32(\mathrm{~m}, 4 \mathrm{H}), 7.29-7.26(\mathrm{~m}, 1 \mathrm{H}), 6.96-6.93(\mathrm{~m}, 4 \mathrm{H}), 6.83-6.81(\mathrm{~m}, 4 \mathrm{H}), 4.87(\mathrm{dd}$, $\mathrm{J}=9.4,3.7 \mathrm{~Hz}, 1 \mathrm{H}), 3.81(\mathrm{dd}, \mathrm{J}=14.5,3.7 \mathrm{~Hz}, 1 \mathrm{H}), 3.77(\mathrm{~s}, 6 \mathrm{H}), 3.71(\mathrm{dd}, \mathrm{J}=14.5,9.4 \mathrm{~Hz}, 1 \mathrm{H}), 2.80$ (br s, $1 \mathrm{H})$

${ }^{13}$ C NMR $\left(125 \mathrm{MHz}, \mathrm{CDCl}_{3}\right) \delta 154.9,142.7,141.7,128.5,127.8,126.1,122.8,114.8,71.2,61.8,55.6$

IR $v_{\max } 3306,2944,2890,2850,2836,1582,1506,1464,1442,1242,1217,1130,1038,1029,916,826 \mathrm{~cm}^{-1}$.

LRMS (ESI): Calcd. for $\mathrm{C}_{22} \mathrm{H}_{23} \mathrm{NNaO}_{3}: \mathrm{m} / \mathrm{z} 372.16\left([\mathrm{M}+\mathrm{Na}]^{+}\right)$, found: $\mathrm{m} / \mathrm{z}(\%)=372.16\left([\mathrm{M}+\mathrm{Na}]^{+}\right)$

HPLC: 70\% ee, ID, 2-propanol: $n$-Hexane $=1: 9$, flow: $1.0 \mathrm{~mL} / \mathrm{min}, 220 \mathrm{~nm}, T_{\mathrm{R}}=15.40 \mathrm{~min}$ (major) and $T_{\mathrm{R}}=16.71 \mathrm{~min}$ (minor).

$[\alpha]^{25}=+6.1\left(c=0.50, \mathrm{CHCl}_{3}\right)$. 
<smiles>O[C@H](CN(c1ccc2ccccc2c1)c1ccc2ccccc2c1)c1ccccc1</smiles>

Purification by column chromatography (gradient elution with 2-9\% ethyl acetate-hexane) afforded the desired product (78 $\mathrm{mg}, 80 \%$ ) as yellow solid.

${ }^{1} \mathbf{H}$ NMR $\left(500 \mathrm{MHz}, \mathrm{CDCl}_{3}\right) \delta 7.68(\mathrm{~d}, \mathrm{~J}=8.0 \mathrm{~Hz}, 2 \mathrm{H}), 7.63(\mathrm{~d}, \mathrm{~J}=3.4 \mathrm{~Hz}, 2 \mathrm{H}), 7.60(\mathrm{~d}, \mathrm{~J}=8.1 \mathrm{~Hz}, 2 \mathrm{H}), 7.38-7.35(\mathrm{~m}, 6 \mathrm{H})$, $7.31-7.28(\mathrm{~m}, 4 \mathrm{H}), 7.26-7.23(\mathrm{~m}, 1 \mathrm{H}), 7.20(\mathrm{dd}, \mathrm{J}=8.9,2.4 \mathrm{~Hz}, 2 \mathrm{H}), 5.01(\mathrm{t}, \mathrm{J}=6.4 \mathrm{~Hz}, 1 \mathrm{H}), 4.06(\mathrm{~d}, \mathrm{~J}=6.5 \mathrm{~Hz}, 2 \mathrm{H}), 2.57$ (br $\mathrm{s}, 1 \mathrm{H})$

${ }^{13}$ C NMR (125MHz, $\left.\mathrm{CDCl}_{3}\right) \delta$ 145.7, 141.9, 134.6, 129.7, 129.1, 128.7, 128.0, 127.6, 127.1, 126.5, 126.2, 124.4, 122.9, $117.1,71.2,60.9$

IR $v_{\max } 3541,3057,3016,2966,2951,2915,1626,1596,1506,1471,1391,1216,1182,963,843,815 \mathrm{~cm}^{-1}$.

LRMS (ESI): Calcd. for $\mathrm{C}_{28} \mathrm{H}_{23} \mathrm{NNaO}: \mathrm{m} / \mathrm{z} 412.17\left([\mathrm{M}+\mathrm{Na}]^{+}\right)$, found: $\mathrm{m} / \mathrm{z}(\%)=412.17\left([\mathrm{M}+\mathrm{Na}]^{+}\right)$

HPLC: $84 \%$ ee, ID, 2-propanol: $n$-Hexane=1:9, flow: $1.0 \mathrm{~mL} / \mathrm{min}, 220 \mathrm{~nm}, T_{\mathrm{R}}=9.05 \mathrm{~min}$ (major) and $T_{\mathrm{R}}=10.36 \mathrm{~min}$ (minor).

$[\alpha]^{25} \mathrm{D}=+94.2\left(c=3.0, \mathrm{CHCl}_{3}\right)$.

\section{(R)-2-(bis(4-bromophenyl)amino)-1-phenylethan-1-ol (6m)}<smiles>OC(CN(c1ccc(Br)cc1)c1ccc(Br)cc1)c1ccccc1</smiles>

Purification by column chromatography (gradient elution with 2-9\% ethyl acetate-hexane) afforded the desired product (102 $\mathrm{mg}, 91 \%)$ as colorless oil.

${ }^{1} \mathbf{H}$ NMR $\left(500 \mathrm{MHz}, \mathrm{CDCl}_{3}\right) \delta 7.33-7.27$ (m, 9H), $6.85-6.82(\mathrm{~m}, 4 \mathrm{H}), 4.85(\mathrm{t}, \mathrm{J}=6.5 \mathrm{~Hz}, 1 \mathrm{H}), 3.80(\mathrm{~d}, \mathrm{~J}=6.5 \mathrm{~Hz}, 2 \mathrm{H}), 2.43$ (br s, 1H)

${ }^{13}$ C NMR $\left(125 \mathrm{MHz}, \mathrm{CDCl}_{3}\right) \delta 146.8,141.6,132.4,128.7,128.2,126.0,123.0,114.6,71.2,60.3$

IR $v_{\max } 3667,2966,2937,2922,2865,2844,1510,1477,1346,1331,1322,1057,1032,1012,762,640 \mathrm{~cm}^{-1}$.

LRMS (ESI): Calcd. for $\mathrm{C}_{20} \mathrm{H}_{17} \mathrm{Br}_{2} \mathrm{NNaO}: \mathrm{m} / \mathrm{z} 469.96$ ([M+Na $\left.]^{+}\right)$, found: $\mathrm{m} / \mathrm{z}(\%)=469.96$ ([M+Na $\left.]^{+}, 100\right), 467.96$ (51), $471.96(48)$

HPLC: $90 \%$ ee, ID, 2-propanol: $n$-Hexane=1:9, flow: $1.0 \mathrm{~mL} / \mathrm{min}, 220 \mathrm{~nm}, T_{\mathrm{R}}=5.66 \mathrm{~min}$ (major) and $T_{\mathrm{R}}=6.32 \mathrm{~min}$ (minor). $[\alpha]^{25}=+78.1\left(c=3.0, \mathrm{CHCl}_{3}\right)$.

(R)-2-(bis(4-bromophenyl)amino)-1-(4-methoxyphenyl)ethan-1-ol (6n) 
<smiles>COc1ccc([C@@H](O)CN(c2ccc(Br)cc2)c2ccc(Br)cc2)cc1</smiles>

Purification by column chromatography (gradient elution with 2-11\% ethyl acetate-hexane) afforded the desired product (112 mg, 94\%) as white solid.

${ }^{1} \mathbf{H}$ NMR $\left(500 \mathrm{MHz}, \mathrm{CDCl}_{3}\right) \delta 7.36-7.31(\mathrm{~m}, 4 \mathrm{H}), 7.26-7.24(\mathrm{~m}, 2 \mathrm{H}), 6.89-6.86(\mathrm{~m}, 6 \mathrm{H}), 4.87(\mathrm{t}, \mathrm{J}=6.4 \mathrm{~Hz}, 1 \mathrm{H}), 3.83(\mathrm{~d}$, $\mathrm{J}=6.4 \mathrm{~Hz}, 2 \mathrm{H}), 3.79$ (s, 3H), 2.26 (br s, 1H)

${ }^{13}$ C NMR $\left(125 \mathrm{MHz}, \mathrm{CDCl}_{3}\right) \delta 159.6,146.9,133.8,132.4,127.3,123.0,114.6,114.1,70.9,60.3,55.4$

IR $v_{\max } 3567,2889,2851,2837,1496,1443,1218,1176,1100,1091,1039,1025,947,904,840,763,624 \mathrm{~cm}^{-1}$.

LRMS (ESI): Calcd. for $\mathrm{C}_{21} \mathrm{H}_{19} \mathrm{Br}_{2} \mathrm{KNO}_{2}: \mathrm{m} / \mathrm{z} 515.94\left([\mathrm{M}+\mathrm{K}]^{+}\right)$, found: $\mathrm{m} / \mathrm{z}(\%)=515.94\left([\mathrm{M}+\mathrm{K}]^{+}, 100\right), 513.95(51)$, $517 . .94(48)$

HPLC: $84 \%$ ee, OZ-H, 2-propanol: $n$-Hexane=1:9, flow: $1.0 \mathrm{~mL} / \mathrm{min}, 220 \mathrm{~nm}, T_{\mathrm{R}}=8.22 \mathrm{~min}$ (minor) and $T_{\mathrm{R}}=13.58 \mathrm{~min}$ (major).

$[\alpha]^{25}=+10.2\left(c=1.0, \mathrm{CHCl}_{3}\right)$.

\section{(R)-2-(bis(4-bromophenyl)amino)-1-(p-tolyl)ethan-1-ol (6o)}<smiles>Cc1ccc([C@@H](O)CN(c2ccc(Br)cc2)c2ccc(Br)cc2)cc1</smiles>

Purification by column chromatography (gradient elution with 2-9\% ethyl acetate-hexane) afforded the desired product (104 mg, 90\%) as white solid.

${ }^{1} \mathbf{H}$ NMR $\left(500 \mathrm{MHz}, \mathrm{CDCl}_{3}\right) \delta 7.33-7.30(\mathrm{~m}, 4 \mathrm{H}), 7.21(\mathrm{~d}, \mathrm{~J}=8.0 \mathrm{~Hz}, 2 \mathrm{H}), 7.14(\mathrm{~d}, \mathrm{~J}=7.8 \mathrm{~Hz}, 2 \mathrm{H}), 6.89-6.86(\mathrm{~m}, 4 \mathrm{H}), 4.86$ (t, J=6.4Hz, 1H), 3.81 (d, J=6.3Hz, 2H), 2.33 (s, 3H), 2.29 (br s, 1H)

${ }^{13}$ C NMR $\left(125 \mathrm{MHz}, \mathrm{CDCl}_{3}\right) \delta 146.9,138.6,138.0,132.4,129.4,126.0,123.1,114.6,71.2,60.3,21.3$

IR $v_{\max } 3568,2981,2973,2939,1488,1455,1346,1321,1216,1055,1032,1012,847,764,680 \mathrm{~cm}^{-1}$.

LRMS (ESI): Calcd. for $\mathrm{C}_{21} \mathrm{H}_{19} \mathrm{Br}_{2} \mathrm{NNaO}: \mathrm{m} / \mathrm{z} 483.97\left([\mathrm{M}+\mathrm{Na}]^{+}\right)$, found: $\mathrm{m} / \mathrm{z}(\%)=483.97\left([\mathrm{M}+\mathrm{Na}]^{+}, 100\right), 481.98(51)$, $485.97(48)$

HPLC: $90 \%$ ee, OZ-H, 2-propanol: $n$-Hexane=1:9, flow: $1.0 \mathrm{~mL} / \mathrm{min}, 220 \mathrm{~nm}, T_{\mathrm{R}}=5.06 \mathrm{~min}$ (minor) and $T_{\mathrm{R}}=6.06 \mathrm{~min}$ (major).

$[\alpha]^{25}=+77.9\left(c=2.0, \mathrm{CHCl}_{3}\right)$.

\section{(R)-2-(9H-carbazol-9-yl)-1-phenylethanol (7a)}


<smiles>O[C@H](Cn1c2ccccc2c2ccccc21)c1ccccc1</smiles>

Purification by column chromatography (gradient elution with 2-9\% ethyl acetate-hexane) afforded the desired product (59 $\mathrm{mg}, 82 \%)$ as white solid.

${ }^{1}$ H NMR $\left(500 \mathrm{MHz}, \mathrm{CDCl}_{3}\right) \delta 8.03(\mathrm{~d}, \mathrm{~J}=7.8 \mathrm{~Hz}, 2 \mathrm{H}), 7.41-7.27(\mathrm{~m}, 9 \mathrm{H}), 7.21-7.18(\mathrm{~m}, 2 \mathrm{H}), 5.03(\mathrm{dd}, \mathrm{J}=8.4,3.7 \mathrm{~Hz}, 1 \mathrm{H})$, $4.42(\mathrm{dd}, \mathrm{J}=15.1,8.5 \mathrm{~Hz}, 1 \mathrm{H}), 4.32(\mathrm{dd}, \mathrm{J}=15.1,3.8 \mathrm{~Hz}, 1 \mathrm{H}), 2.07$ (br s, 1H)

${ }^{13}$ C NMR $\left(125 \mathrm{MHz}, \mathrm{CDCl}_{3}\right) \delta 141.3,140.8,128.8,128.3,125.9,125.9,123.1,120.4,119.3,109.1,73.4,51.4$

IR $v_{\max } 3546,3057,3025,1627,1598,1484,1458,1348,1326,1216,1025,916,848,749 \mathrm{~cm}^{-1}$.

HRMS (ESI, positive mode): Calcd. for $\mathrm{C}_{20} \mathrm{H}_{17} \mathrm{NONa}: \mathrm{m} / \mathrm{z} 310.1202\left([\mathrm{M}+\mathrm{Na}]^{+}\right)$, found: $\mathrm{m} / \mathrm{z} 310.1203\left([\mathrm{M}+\mathrm{Na}]^{+}\right)$

HPLC: $90 \%$ ee, IA, 2-propanol: $n$-Hexane=1:9, flow: $1.0 \mathrm{~mL} / \mathrm{min}, 220 \mathrm{~nm}, T_{\mathrm{R}}=7.85 \mathrm{~min}$ (minor) and $T_{\mathrm{R}}=8.84$ min (major). $[\alpha]^{25} \mathrm{D}=+5.0\left(c=1.6, \mathrm{CHCl}_{3}\right)$.

\section{(R)-2-(9H-carbazol-9-yl)-1-p-tolylethanol (7b)}<smiles>Cc1ccc(C(O)Cn2c3ccccc3c3ccccc32)cc1</smiles>

Purification by column chromatography (gradient elution with 2-9\% ethyl acetate-hexane) afforded the desired product (75 $\mathrm{mg}, 99 \%)$ as white solid.

${ }^{1} \mathbf{H}$ NMR $\left(500 \mathrm{MHz}, \mathrm{CDCl}_{3}\right) \delta 8.02(\mathrm{~d}, \mathrm{~J}=7.6 \mathrm{~Hz}, 2 \mathrm{H}), 7.42-7.39(\mathrm{~m}, 2 \mathrm{H}), 7.37(\mathrm{~d}, \mathrm{~J}=7.6 \mathrm{~Hz}, 2 \mathrm{H}), 7.25(\mathrm{~d}, \mathrm{~J}=7.9 \mathrm{~Hz}, 2 \mathrm{H})$, $7.21-7.18(\mathrm{~m}, 2 \mathrm{H}), 7.12(\mathrm{~d}, \mathrm{~J}=7.9 \mathrm{~Hz}, 2 \mathrm{H}), 5.00(\mathrm{dd}, \mathrm{J}=8.6,3.3 \mathrm{~Hz}, 1 \mathrm{H}), 4.40(\mathrm{dd}, \mathrm{J}=15.0,8.7 \mathrm{~Hz}, 1 \mathrm{H}), 4.29(\mathrm{dd}, \mathrm{J}=15.1$, $3.4 \mathrm{~Hz}, 1 \mathrm{H}), 2.31(\mathrm{~s}, 3 \mathrm{H}), 2.02(\mathrm{br} \mathrm{s}, 1 \mathrm{H})$

${ }^{13}$ C NMR $\left(125 \mathrm{MHz}, \mathrm{CDCl}_{3}\right) \delta 140.8,138.4,138.0,129.5,125.8(2 \mathrm{C}), 123.1,120.4,119.3,109.2,73.3,51.5,21.3$

IR $v_{\max } 3552,3051,3021,2929,2885,1627,1597,1514,1484,1459,1325,1192,816,788 \mathrm{~cm}^{-1}$.

LRMS (ESI): Calcd. for $\mathrm{C}_{21} \mathrm{H}_{19} \mathrm{KNO}: \mathrm{m} / \mathrm{z} 340.11\left([\mathrm{M}+\mathrm{K}]^{+}\right)$, found: $\mathrm{m} / \mathrm{z}(\%)=340.11\left([\mathrm{M}+\mathrm{K}]^{+}\right)$

HPLC: $88 \%$ ee, IA, 2-propanol: $n$-Hexane $=1: 9$, flow: $1.0 \mathrm{~mL} / \mathrm{min}, 220 \mathrm{~nm}, T_{\mathrm{R}}=8.33 \mathrm{~min}(\operatorname{minor})$ and $T_{\mathrm{R}}=10.04 \mathrm{~min}$ (major).

$[\alpha]^{25} \mathrm{D}=+3.8\left(c=2.0, \mathrm{CHCl}_{3}\right)$.

\section{(R)-1-(4-bromophenyl)-2-(9H-carbazol-9-yl)ethanol (7c)}<smiles>O[C@H](Cn1c2ccccc2c2ccccc21)c1ccc(Br)cc1</smiles>

Purification by column chromatography (gradient elution with 2-9\% ethyl acetate-hexane) afforded the desired product (82 $\mathrm{mg}, 90 \%)$ as white solid. 
${ }^{1} \mathbf{H}$ NMR $\left(500 \mathrm{MHz}, \mathrm{CDCl}_{3}\right) \delta 8.08(\mathrm{~d}, \mathrm{~J}=7.8 \mathrm{~Hz}, 2 \mathrm{H}), 7.49-7.42(\mathrm{~m}, 4 \mathrm{H}), 7.39(\mathrm{~d}, \mathrm{~J}=8.1 \mathrm{~Hz}, 2 \mathrm{H}), 7.30-7.29(\mathrm{~m}, 2 \mathrm{H}), 7.25-$ $7.22(\mathrm{~m}, 2 \mathrm{H}), 5.14(\mathrm{dd}, \mathrm{J}=8.4,3.9 \mathrm{~Hz}, 1 \mathrm{H}), 4.46(\mathrm{dd}, \mathrm{J}=15.0,8.5 \mathrm{~Hz}, 1 \mathrm{H}), 4.37$ (dd, J=15.0, 4.0Hz, 1H), 2.11 (br s, $1 \mathrm{H})$ ${ }^{13}$ C NMR $\left(125 \mathrm{MHz}, \mathrm{CDCl}_{3}\right) \delta 140.7,140.2,131.9,127.6,125.9,123.1,122.1,120.4,119.4,108.9,72.8,51.2$

IR $v_{\max } 3526,3052,3021,2965,1626,1595,1485,1458,1379,1348,1216,1191,1072,927,853,644 \mathrm{~cm}^{-1}$.

HRMS (ESI, positive mode): Calcd. for $\mathrm{C}_{20} \mathrm{H}_{16} \mathrm{BrNONa}$ : m/z $388.0307\left([\mathrm{M}+\mathrm{Na}]^{+}\right)$, found: $\mathrm{m} / \mathrm{z} 388.0308\left([\mathrm{M}+\mathrm{Na}]^{+}\right)$ HPLC: $87 \%$ ee, IA, 2-propanol: $n$-Hexane $=1: 9$, flow: $1.0 \mathrm{~mL} / \mathrm{min}, 220 \mathrm{~nm}, T_{\mathrm{R}}=8.66 \mathrm{~min}(\operatorname{minor})$ and $T_{\mathrm{R}}=10.34 \mathrm{~min}$ (major).

$[\alpha]^{25}=+16.8\left(c=2.0, \mathrm{CHCl}_{3}\right)$.

\section{(R)-2-(9H-carbazol-9-yl)-1-(4-chlorophenyl)ethan-1-ol (7d)}<smiles>O[C@H](Cn1c2ccccc2c2ccccc21)c1ccc(Cl)cc1</smiles>

Purification by column chromatography (gradient elution with 2-11\% ethyl acetate-hexane) afforded the desired product (71 $\mathrm{mg}, 88 \%)$ as white solid.

${ }^{1} \mathbf{H}$ NMR $\left(500 \mathrm{MHz}, \mathrm{CDCl}_{3}\right) \delta 8.05(\mathrm{~d}, \mathrm{~J}=6.9 \mathrm{~Hz}, 2 \mathrm{H}), 7.43-7.40(\mathrm{~m}, 2 \mathrm{H}), 7.34(\mathrm{~d}, \mathrm{~J}=8.2 \mathrm{~Hz}, 2 \mathrm{H}), 7.29(\mathrm{~s}, 4 \mathrm{H}), 7.23-7.20$ $(\mathrm{m}, 2 \mathrm{H}), 5.06(\mathrm{dd}, \mathrm{J}=8.4,4.0 \mathrm{~Hz}, 1 \mathrm{H}), 4.40(\mathrm{dd}, \mathrm{J}=15.1,8.5 \mathrm{~Hz}, 1 \mathrm{H}), 4.31$ (dd, J=15.0, 4.0Hz, 1H), 2.12 (br s, 1H)

${ }^{13}$ C NMR $\left(125 \mathrm{MHz}, \mathrm{CDCl}_{3}\right) \delta 140.7,139.8,134.1,129.0,127.3,126.0,123.1,120.5,119.5,109.0,72.8,51.3$ IR $v_{\max } 3578,2931,2890,2851,1597,1485,1459,1350,1326,1217,1154,1092,1014,849,827,723,668 \mathrm{~cm}^{-1}$. LRMS (ESI): Calcd. for $\mathrm{C}_{20} \mathrm{H}_{16} \mathrm{ClNNaO}: \mathrm{m} / \mathrm{z} 344.08\left([\mathrm{M}+\mathrm{Na}]^{+}\right)$, found: $\mathrm{m} / \mathrm{z}(\%)=344.08\left([\mathrm{M}+\mathrm{Na}]^{+}, 100\right), 346.08(32)$ HPLC: $90 \%$ ee, ID, 2-propanol: $n$-Hexane=1:9, flow: $1.0 \mathrm{~mL} / \mathrm{min}, 220 \mathrm{~nm}, T_{\mathrm{R}}=6.30 \mathrm{~min}$ (minor) and $T_{\mathrm{R}}=6.62 \mathrm{~min}$ (major). $[\alpha]^{25} \mathrm{D}=+12.5\left(c=1.0, \mathrm{CHCl}_{3}\right)$.

\section{(R)-2-(9H-carbazol-9-yl)-1-o-tolylethanol (7e)}<smiles>Cc1ccccc1[C@@H](O)Cn1c2ccccc2c2ccccc21</smiles>

Purification by column chromatography (gradient elution with 2-9\% ethyl acetate-hexane) afforded the desired product (74 $\mathrm{mg}, 98 \%)$ as white solid.

${ }^{1} \mathbf{H}$ NMR $\left(500 \mathrm{MHz}, \mathrm{CDCl}_{3}\right) \delta 8.03(\mathrm{~d}, \mathrm{~J}=7.7 \mathrm{~Hz}, 2 \mathrm{H}), 7.62(\mathrm{~d}, \mathrm{~J}=7.7 \mathrm{~Hz}, 1 \mathrm{H}), 7.42-7.37(\mathrm{~m}, 4 \mathrm{H}), 7.25-7.23(\mathrm{~m}, 1 \mathrm{H}), 7.21$ - $7.17(\mathrm{~m}, 3 \mathrm{H}), 7.09$ (d, J=7.5Hz, 1H), $5.32(\mathrm{dd}, \mathrm{J}=8.5,3.8 \mathrm{~Hz}, 1 \mathrm{H}), 4.42(\mathrm{dd}, \mathrm{J}=15.1,8.6 \mathrm{~Hz}, 1 \mathrm{H}), 4.31(\mathrm{dd}, \mathrm{J}=15.1,3.9 \mathrm{~Hz}$, $1 \mathrm{H}), 2.27(\mathrm{~s}, 3 \mathrm{H}), 2.00($ br s, $1 \mathrm{H})$

${ }^{13}$ C NMR $\left(125 \mathrm{MHz}, \mathrm{CDCl}_{3}\right) \delta 140.9,139.6,134.6,130.7,128.1,126.8,125.9,125.8,123.1,120.4,119.4,109.1,69.9,50.3$, 19.4

IR $v_{\max } 3545,3011,2967,2924,1627,1597,1484,1347,1325,1215,1154,1052,1033,905,834,749 \mathrm{~cm}^{-1}$.

LRMS (ESI): Calcd. for $\mathrm{C}_{21} \mathrm{H}_{19} \mathrm{NNaO}: \mathrm{m} / \mathrm{z} 324.14\left([\mathrm{M}+\mathrm{Na}]^{+}\right)$, found: $\mathrm{m} / \mathrm{z}(\%)=324.13\left([\mathrm{M}+\mathrm{Na}]^{+}\right)$

HPLC: $87 \%$ ee, IA-3, 2-propanol: $n$-Hexane $=1: 9$, flow: $1.0 \mathrm{~mL} / \mathrm{min}, 220 \mathrm{~nm}, T_{\mathrm{R}}=7.01 \mathrm{~min}(\operatorname{minor})$ and $T_{\mathrm{R}}=9.07 \mathrm{~min}$ (major). 
$[\alpha]^{25} \mathrm{D}=-31.4\left(c=2.0, \mathrm{CHCl}_{3}\right)$.

\section{(S)-2-(9H-carbazol-9-yl)-1-(furan-2-yl)ethan-1-ol (7f)}<smiles>OC(Cn1c2ccccc2c2ccccc21)c1ccco1</smiles>

Purification by column chromatography (gradient elution with 2-9\% ethyl acetate-hexane) afforded the desired product (45 $\mathrm{mg}, 65 \%)$ as white solid.

${ }^{1} \mathrm{H}$ NMR $\left(500 \mathrm{MHz}, \mathrm{CDCl}_{3}\right) \delta 8.05(\mathrm{~d}, \mathrm{~J}=7.6 \mathrm{~Hz}, 2 \mathrm{H}), 7.43-7.37(\mathrm{~m}, 5 \mathrm{H}), 7.23-7.19(\mathrm{~m}, 2 \mathrm{H}), 6.29(\mathrm{dd}, \mathrm{J}=3.2,1.9 \mathrm{~Hz}, 1 \mathrm{H})$, $6.21(\mathrm{~d}, \mathrm{~J}=3.2 \mathrm{~Hz}, 1 \mathrm{H}), 5.14(\mathrm{dd}, \mathrm{J}=7.4,5.0 \mathrm{~Hz}, 1 \mathrm{H}), 4.62(\mathrm{dd}, \mathrm{J}=15.1,7.4 \mathrm{~Hz}, 1 \mathrm{H}), 4.58(\mathrm{dd}, \mathrm{J}=15.1,5.0 \mathrm{~Hz}, 1 \mathrm{H}), 2.20$ (br s,

1H)

${ }^{13}$ C NMR $\left(125 \mathrm{MHz}, \mathrm{CDCl}_{3}\right) \delta 153.5,142.6,140.8,125.9,123.1,120.4,119.4,110.7,109.0,107.5,67.3,48.3$

IR $v_{\max } 3688,2944,2889,2851,2837,1597,1485,1460,1352,1326,1216,1153,1058,884,808 \mathrm{~cm}^{-1}$.

LRMS (ESI): Calcd. for $\mathrm{C}_{18} \mathrm{H}_{16} \mathrm{LiNO}_{2}: \mathrm{m} / \mathrm{z} 285.13\left([\mathrm{M}+\mathrm{H}+\mathrm{Li}]^{+}\right)$, found: $\mathrm{m} / \mathrm{z}(\%)=285.15\left([\mathrm{M}+\mathrm{H}+\mathrm{Li}]^{+}\right)$

HPLC: $88 \%$ ee, ID, 2-propanol: $n$-Hexane=1:9, flow: $1.0 \mathrm{~mL} / \mathrm{min}, 220 \mathrm{~nm}, T_{\mathrm{R}}=7.58 \mathrm{~min}$ (major) and $T_{\mathrm{R}}=8.48 \mathrm{~min}$ (minor). $[\alpha]^{25}=+10.0\left(c=1.0, \mathrm{CHCl}_{3}\right)$.

\section{(S)-2-(9H-carbazol-9-yl)-1-(thiophen-2-yl)ethan-1-ol (7g)}<smiles>O[C@@H](Cn1c2ccccc2c2ccccc21)c1cccs1</smiles>

Purification by column chromatography (gradient elution with 2-9\% ethyl acetate-hexane) afforded the desired product (69 mg, 94\%) as white solid.

${ }^{1} \mathbf{H}$ NMR $\left(500 \mathrm{MHz}, \mathrm{CDCl}_{3}\right) \delta 8.03(\mathrm{~d}, \mathrm{~J}=7.8 \mathrm{~Hz}, 2 \mathrm{H}), 7.41-7.38(\mathrm{~m}, 2 \mathrm{H}), 7.35(\mathrm{~d}, \mathrm{~J}=8.1 \mathrm{~Hz}, 2 \mathrm{H}), 7.23-7.17(\mathrm{~m}, 3 \mathrm{H}), 6.91$ - 6.90 (m, 2H), 5.31 (dd, J=8.4, 4.0Hz, 1H), 4.51 (dd, J=15.0, 8.5Hz, 1H), 4.41 (dd, J=15.0, 3.9Hz, 1H), 2.28 (br s, 1H)

${ }^{13}$ C NMR (125MHz, $\left.\mathrm{CDCl}_{3}\right) \delta$ 144.7, 140.8, 127.1, 125.9, 125.2, 124.3, 123.1, 120.4, 119.4, 109.1, 69.7, 51.4 IR $v_{\max } 3521,3055,2885,1627,1598,1485,1459,1351,1326,1252,1217,1082,1038,1027,928,855 \mathrm{~cm}^{-1}$.

LRMS (ESI): Calcd. for $\mathrm{C}_{18} \mathrm{H}_{15} \mathrm{KNOS}: \mathrm{m} / \mathrm{z} 332.05\left([\mathrm{M}+\mathrm{K}]^{+}\right)$, found: $\mathrm{m} / \mathrm{z}(\%)=332.05\left([\mathrm{M}+\mathrm{K}]^{+}\right)$

HPLC: $89 \%$ ee, IA-3, 2-propanol: $n$-Hexane $=1: 9$, flow: $1.0 \mathrm{~mL} / \mathrm{min}, 220 \mathrm{~nm}, T_{\mathrm{R}}=9.55 \mathrm{~min}$ (minor) and $T_{\mathrm{R}}=11.18 \mathrm{~min}$ (major).

$[\alpha]^{25}=+18.9\left(c=1.0, \mathrm{CHCl}_{3}\right)$.

(R)-2-(9H-carbazol-9-yl)-1-(naphthalen-1-yl)ethan-1-ol (7h)<smiles>OC(Cn1c2ccccc2c2ccccc21)c1cccc2ccccc12</smiles> 
Purification by column chromatography (gradient elution with 2-9\% ethyl acetate-hexane) afforded the desired product (74 $\mathrm{mg}, 88 \%)$ as white solid.

${ }^{1} \mathbf{H}$ NMR $\left(500 \mathrm{MHz}, \mathrm{CDCl}_{3}\right) \delta 8.23(\mathrm{~d}, \mathrm{~J}=8.4 \mathrm{~Hz}, 1 \mathrm{H}), 8.06(\mathrm{~d}, \mathrm{~J}=7.7 \mathrm{~Hz}, 2 \mathrm{H}), 7.89(\mathrm{~d}, \mathrm{~J}=8.2 \mathrm{~Hz}, 1 \mathrm{H}), 7.80(\mathrm{~d}, \mathrm{~J}=8.2 \mathrm{~Hz}, 1 \mathrm{H})$, $7.74(\mathrm{~d}, \mathrm{~J}=7.1 \mathrm{~Hz}, 1 \mathrm{H}), 7.60-7.57(\mathrm{~m}, 1 \mathrm{H}), 7.53-7.50(\mathrm{~m}, 1 \mathrm{H}), 7.47-7.41(\mathrm{~m}, 5 \mathrm{H}), 7.22(\mathrm{t}, \mathrm{J}=7.1 \mathrm{~Hz}, 2 \mathrm{H}), 5.88(\mathrm{dd}, \mathrm{J}=8.1$, $4.0 \mathrm{~Hz}, 1 \mathrm{H}), 4.62(\mathrm{dd}, \mathrm{J}=15.5,8.2 \mathrm{~Hz}, 1 \mathrm{H}), 4.58(\mathrm{dd}, \mathrm{J}=15.6,4.1 \mathrm{~Hz}, 1 \mathrm{H}), 2.19$ (br s, 1H)

${ }^{13}$ C NMR (125MHz, $\left.\mathrm{CDCl}_{3}\right)$ 141.0, 137.1, 134.0, 130.5, 129.3, 128.8, 126.6, 125.9, 125.9, 125.7, 124.0, 123.2, 122.7, 120.5, $119.4,109.2,71.1,50.6$

IR $v_{\max } 3527,3017,2960,2932,2865,1627,1596,1484,1460,1394,1241,1216,1096,1031,907,833,793 \mathrm{~cm}^{-1}$.

LRMS (ESI): Calcd. for $\mathrm{C}_{24} \mathrm{H}_{19} \mathrm{NNaO}: \mathrm{m} / \mathrm{z} 360.14\left([\mathrm{M}+\mathrm{Na}]^{+}\right)$, found: $\mathrm{m} / \mathrm{z}(\%)=360.14\left([\mathrm{M}+\mathrm{Na}]^{+}\right)$

HPLC: $92 \%$ ee, ID, 2-propanol: $n$-Hexane $=1: 9$, flow: $1.0 \mathrm{~mL} / \mathrm{min}, 220 \mathrm{~nm}, T_{\mathrm{R}}=8.30 \mathrm{~min}$ (major) and $T_{\mathrm{R}}=10.29 \mathrm{~min}$ (minor).

$[\alpha]^{25} \mathrm{D}=-43.2\left(c=1.0, \mathrm{CHCl}_{3}\right)$. 


\section{Radical Trapping Experiment}

\subsection{General Procedure for Radical Trapping Experiment}<smiles>O=CC1=CCCCC1</smiles>

$1 \mathrm{a}$<smiles>CS(=O)(=O)CN(c1ccccc1)c1ccccc1</smiles>

$2 a$

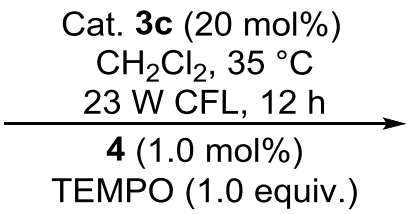

TEMPO (1.0 equiv.)<smiles>CS(=O)(=O)NC[C@@H](O[N+]#N)C1=CCCCC1</smiles>

Not Detected<smiles>CC1(C)CCCC(C)(C)N1OCN(c1ccccc1)c1ccccc1</smiles>

Detected via ESI-MS

To a catalyst 3 solution prepared as described above in dichloromethane was successively added $\left[\operatorname{Ir}(\mathrm{dtbbpy})(\mathrm{ppy})_{2}\right]\left[\mathrm{BF} \mathrm{F}_{4}\right]$ (0.0025 mmol, 0.010 equiv.), 1a (0.38 mmol, 1.5 equiv.), 2a ( $0.25 \mathrm{mmol}, 1.0$ equiv.) and TEMPO (0.25 mmol, 1.0 equiv.) at room temperature. The resulting mixture was irradiated with a $23 \mathrm{~W}$ compact fluorescent light bulb for $12 \mathrm{~h}$ at $35^{\circ} \mathrm{C}$. After completion of the reaction, reaction was quenched with distilled water $(5.0 \mathrm{~mL})$ and extracted with ethyl acetate $(3 \mathrm{x} 5.0$ $\mathrm{mL}$ ). The combined extract was dried over anhydrous $\mathrm{Na}_{2} \mathrm{SO}_{4}$, filtered and concentrated in vacuo. The crude mixture was analyzed by ${ }^{1} \mathrm{H}$ NMR spectroscopy and ESI-MS analysis. The LRMS spectrum shows a peak corresponding to coupled product between $\alpha$-aminoalkyl radical and TEMPO radical.

LRMS (ESI): Calcd. for $\mathrm{C}_{22} \mathrm{H}_{31} \mathrm{~N}_{2} \mathrm{O}: \mathrm{m} / \mathrm{z} 339.24\left([\mathrm{M}+\mathrm{H}]^{+}\right)$, found: $\mathrm{m} / \mathrm{z}(\%)=339.25\left([\mathrm{M}+\mathrm{H}]^{+}\right)$

\subsection{Procedure for Asymmetric Roskamp Reaction in presence of TEMPO}

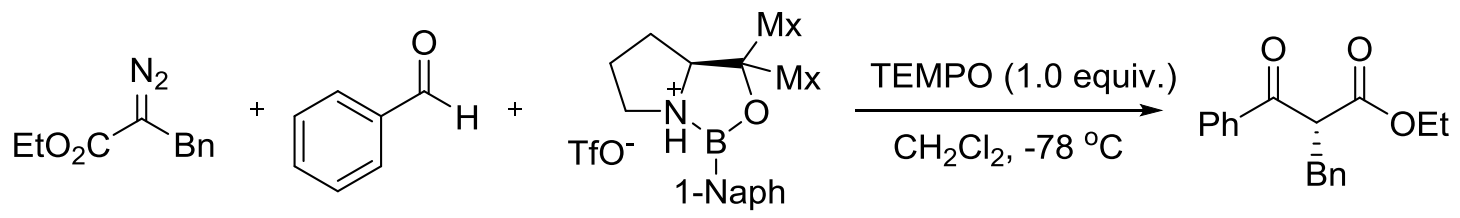

\section{Reported : $\mathbf{8 2} \%$ yield, $\mathbf{8 7 \%}$ ee \\ Result : $\mathbf{8 2} \%$ yield, $\mathbf{9 1 \%}$ ee}

To a catalyst 3 solution prepared as described above in $1.0 \mathrm{~mL}$ of dichloromethane was successively added benzaldehyde (0.38 mmol, 1.5 equiv.), diazoester compound ( $0.25 \mathrm{mmol}, 1.0$ equiv.) and TEMPO ( $0.25 \mathrm{mmol}, 1.0$ equiv.) at $-78{ }^{\circ} \mathrm{C}$. The resulting mixture was stirred at $-78^{\circ} \mathrm{C}$ until complete consumption of diazoester, and then transferred onto silica gel $\left(-78{ }^{\circ} \mathrm{C}\right)$ with a pre-cold pipette. Elution with $9 \%$ ethyl acetate in $n$-hexane $\left(-78^{\circ} \mathrm{C}\right)$ yielded the desired $\alpha$-benzyl- $\beta$-keto esters $(58 \mathrm{mg}$, $82 \%$ ). Desired product was obtained without loss of yield, ${ }^{6}$ COBI was not interrupted by TEMPO. 


\section{Radical Clock Experiment}

To find out the generation of ketyl radicals in reaction pathway, a radical clock experiment was performed using cyclopropyl-substituted aldehyde compounds under the general reaction conditions.

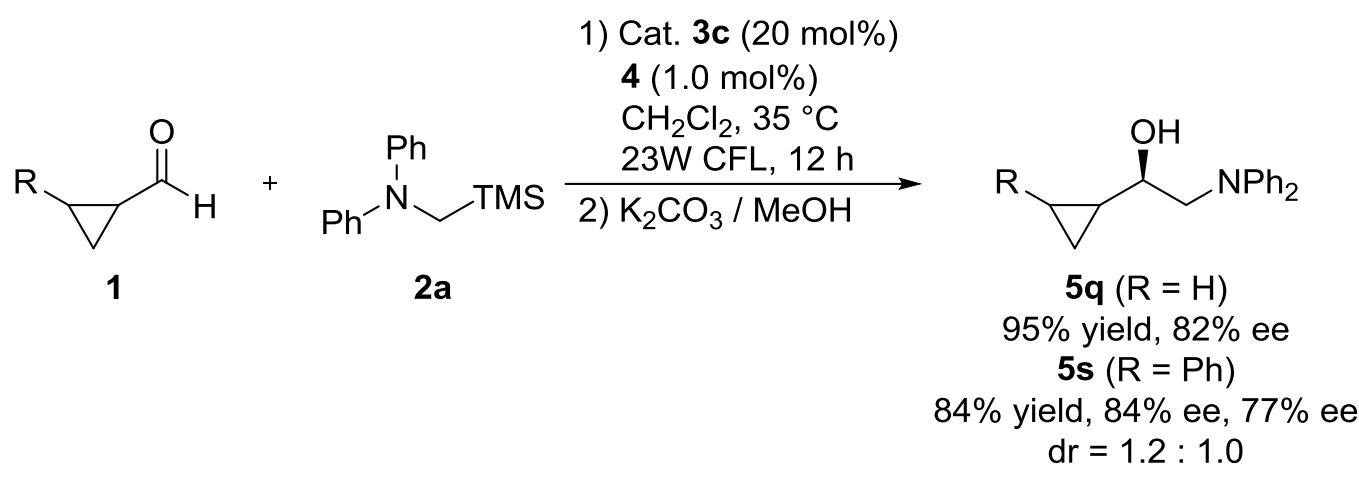

To a catalyst 3 solution prepared as described above in $1.0 \mathrm{~mL}$ of dichloromethane was successively added [Ir(dtbbpy)(ppy) $\left.)_{2}\right]\left[\mathrm{BF}_{4}\right] 4$ (0.0025 mmol, 0.010 equiv.), cyclopropyl-substituted aldehyde compounds (0.38 mmol, 1.5 equiv.) and $\alpha$-silyl amine $2 \mathrm{a}(0.25 \mathrm{mmol}, 1.0$ equiv.) at room temperature. The resulting mixture was irradiated with a $23 \mathrm{~W}$ compact fluorescent light bulb for $12 \mathrm{~h}$ at $35^{\circ} \mathrm{C}$. After completion of the reaction, the reaction was quenched with $3.0 \mathrm{~mL}$ $\mathrm{K}_{2} \mathrm{CO}_{3} / \mathrm{MeOH}$ (saturated). After stirring for $2 \mathrm{~h}$ at room temperature, volatile materials were evaporated in vacuo and distilled water $(5.0 \mathrm{~mL})$ and ethyl acetate $(5.0 \mathrm{~mL})$ were added. The aqueous layer was extracted with ethyl acetate $(3 \times 5.0$ $\mathrm{mL}$ ). The combined extract was dried over anhydrous $\mathrm{Na}_{2} \mathrm{SO}_{4}$, filtered and concentrated in vacuo. The residue was purified by column chromatography (gradient elution with $2-6 \%$ ethyl acetate-hexane) to afford desired $\beta$-amino alcohol. Since ringopened side products were not produced, it is assumed that the ketyl radicals are not involved.

\section{(R)-1-cyclopropyl-2-(diphenylamino)ethan-1-ol (5q)}<smiles>OC(CNc1ccccc1)C1CC1</smiles>

Purification by column chromatography (gradient elution with 2-6\% ethyl acetate-hexane) afforded the desired product (60 mg, $95 \%)$ as white solid.

${ }^{1} \mathrm{H}$ NMR $\left(500 \mathrm{MHz}, \mathrm{CDCl}_{3}\right) \delta 7.27-7.23(\mathrm{~m}, 4 \mathrm{H}), 7.06(\mathrm{~d}, \mathrm{~J}=7.5 \mathrm{~Hz}, 4 \mathrm{H}), 6.97-6.94(\mathrm{~m}, 2 \mathrm{H}), 3.99(\mathrm{dd}, \mathrm{J}=14.9,3.8 \mathrm{~Hz}$, $1 \mathrm{H}), 3.80$ (dd, J=14.9, 8.6Hz, 1H), 3.22 (ddd, J=8.5, 8.5, 3.7Hz, 1H), 2.08 (br s, 1H), $0.92-0.87$ (m, 1H), $0.54-0.50$ (m, $2 \mathrm{H}), 0.34-0.32(\mathrm{~m}, 1 \mathrm{H}), 0.26-0.23(\mathrm{~m}, 1 \mathrm{H})$

${ }^{13} \mathbf{C}$ NMR $\left(125 \mathrm{MHz}, \mathrm{CDCl}_{3}\right) \delta 148.6,129.4,121.8,121.5,73.6,58.8,15.2,2.6,2.4$

IR $v_{\max } 3666,2967,2940,2923,2866,1516,1477,1455,1346,1322,1216,1055,1032,1012 \mathrm{~cm}^{-1}$

HRMS (ESI, positive mode): Calcd. for $\mathrm{C}_{17} \mathrm{H}_{19} \mathrm{NONa}$ m/z $276.1361\left([\mathrm{M}+\mathrm{Na}]^{+}\right)$, found: $\mathrm{m} / \mathrm{z} 276.1359\left([\mathrm{M}+\mathrm{Na}]^{+}\right)$ HPLC: $82 \%$ ee, ID, 2-propanol: $n$-Hexane=5:95, flow: $1.0 \mathrm{~mL} / \mathrm{min}, 220 \mathrm{~nm}, T_{\mathrm{R}}=8.12 \mathrm{~min}$ (major) and $T_{\mathrm{R}}=8.68 \mathrm{~min}$ (minor).

$[\alpha]^{25}=+34.9\left(c=1.0, \mathrm{CHCl}_{3}\right)$.

\section{(1R)-2-(diphenylamino)-1-(2-phenylcyclopropyl)ethan-1-ol (5s)}<smiles>OC(CNc1ccccc1)C1CC1c1ccccc1</smiles> 
Purification by column chromatography (gradient elution with 2-6\% ethyl acetate-hexane) afforded the desired product (69 $\mathrm{mg}, 84 \%$ ) as brown oil. The diastereomeric ratio was determined to be $1.2: 1.0$ by NMR analysis of the crude reaction mixture.

\section{Major diastereomer:}

${ }^{1} \mathbf{H}$ NMR $\left(500 \mathrm{MHz}, \mathrm{CDCl}_{3}\right) \delta 7.28-7.23(\mathrm{~m}, 7 \mathrm{H}), 7.16-7.13(\mathrm{~m}, 1 \mathrm{H}), 7.07(\mathrm{~d}, \mathrm{~J}=7.2 \mathrm{~Hz}, 5 \mathrm{H}), 6.98-6.95(\mathrm{~m}, 2 \mathrm{H}), 4.02$ $(\mathrm{dd}, \mathrm{J}=14.9,3.8 \mathrm{~Hz}, 1 \mathrm{H}), 3.84(\mathrm{dd}, \mathrm{J}=14.9,8.6 \mathrm{~Hz}, 1 \mathrm{H}), 3.59-3.54(\mathrm{~m}, 1 \mathrm{H}), 2.04$ (d, J=2.8Hz, 1H), 1.98 (dt, J=8.7, 4.7Hz, $1 \mathrm{H}), 1.27-1.22(\mathrm{~m}, 1 \mathrm{H}), 1.00-0.92(\mathrm{~m}, 2 \mathrm{H})$

${ }^{13}$ C NMR $\left(125 \mathrm{MHz}, \mathrm{CDCl}_{3}\right) \delta 148.6,142.5,129.5,128.5,126.1,125.8,122.0,121.5,72.2,58.7,26.7,20.6,13.0$

IR $v_{\max } 3557,3008,2953,2924,2857,1602,1589,1497,1462,1364,1249,1218,1068,1033 \mathrm{~cm}^{-1}$.

HRMS (ESI, positive mode): Calcd. for $\mathrm{C}_{23} \mathrm{H}_{23} \mathrm{NONa}: \mathrm{m} / \mathrm{z} 352.1674\left([\mathrm{M}+\mathrm{Na}]^{+}\right)$, found: $\mathrm{m} / \mathrm{z} 352.1672\left([\mathrm{M}+\mathrm{Na}]^{+}\right)$

HPLC: $84 \%$ ee, IA-3, 2-propanol: $n$-Hexane=1:9, flow: $1.0 \mathrm{~mL} / \mathrm{min}, 220 \mathrm{~nm}, T_{\mathrm{R}}=9.02 \mathrm{~min}(\operatorname{minor})$ and $T_{\mathrm{R}}=9.41 \mathrm{~min}$ (major).

$[\alpha]^{25} \mathrm{D}=-11.2\left(c=0.50, \mathrm{CHCl}_{3}\right)$.

\section{Minor diastereomer:}

${ }^{1}$ H NMR $\left(500 \mathrm{MHz}, \mathrm{CDCl}_{3}\right) \delta 7.26-7.22(\mathrm{~m}, 6 \mathrm{H}), 7.17-7.13(\mathrm{~m}, 1 \mathrm{H}), 7.05-7.02(\mathrm{~m}, 6 \mathrm{H}), 6.98-6.94(\mathrm{~m}, 2 \mathrm{H}), 4.02(\mathrm{dd}$, $\mathrm{J}=14.9,3.5 \mathrm{~Hz}, 1 \mathrm{H}), 3.82(\mathrm{dd}, \mathrm{J}=14.9,8.9 \mathrm{~Hz}, 1 \mathrm{H}), 3.56(\mathrm{ddd}, \mathrm{J}=8.9,7.4,3.6 \mathrm{~Hz}, 1 \mathrm{H}), 2.13$ (br s, 1H), $1.88-1.85$ (m, $1 \mathrm{H}$ ), $1.28-1.24(\mathrm{~m}, 1 \mathrm{H}), 1.10-1.06(\mathrm{~m}, 1 \mathrm{H}), 0.99-0.95(\mathrm{~m}, 1 \mathrm{H})$

${ }^{13} \mathrm{C}$ NMR $\left(125 \mathrm{MHz}, \mathrm{CDCl}_{3}\right) \delta 148.6,142.3,129.5,128.5,126.0,125.9,122.1,121.6,72.0,58.9,26.7,20.5,13.2$ IR $v_{\max } 3427,3024,2956,2926,2855,1602,1589,1496,1462,1365,1248,1218,1070,1031 \mathrm{~cm}^{-1}$.

HRMS (ESI, positive mode): Calcd. for $\mathrm{C}_{23} \mathrm{H}_{23} \mathrm{NONa}: \mathrm{m} / \mathrm{z} 352.1674\left([\mathrm{M}+\mathrm{Na}]^{+}\right)$, found: $\mathrm{m} / \mathrm{z} 352.1672\left([\mathrm{M}+\mathrm{Na}]^{+}\right)$ HPLC: 77\% ee, IA-3, 2-propanol: $n$-Hexane $=1: 9$, flow: $1.0 \mathrm{~mL} / \mathrm{min}, 220 \mathrm{~nm}, T_{\mathrm{R}}=7.18 \mathrm{~min}(\operatorname{minor})$ and $T_{\mathrm{R}}=8.21 \mathrm{~min}$ (major).

$[\alpha]^{25}=+87.0\left(c=0.50, \mathrm{CHCl}_{3}\right)$. 


\section{Light On/Off Experiment}

To a catalyst $3 \mathbf{c}$ or $3 \mathbf{d}$ solution prepared as described above in $1.0 \mathrm{~mL}$ of $\mathrm{CD}_{2} \mathrm{Cl}_{2}$ was successively added [Ir(dtbbpy)(ppy) $\left.)_{2}\right]\left[\mathrm{BF}_{4}\right] 4$ (0.0025 mmol, 0.010 equiv.), 1 (0.38 mmol, 1.5 equiv.) and $\alpha$-silyl amine 2a (0.25 mmol, 1.0 equiv.) in NMR tube at room temperature. The resulting mixture was irradiated with a $23 \mathrm{~W}$ compact fluorescent light bulb during “on" periods, and placed under dark during "off” periods. At each time of measurement was quickly done by NMR spectrometer. The ${ }^{1} \mathrm{H}$ NMR yield of $5 \mathbf{a}$ and $\mathbf{6 a}$ were based on dichloromethane as an internal standard. The reactions do not proceeded further when irradiation is stopped, which supports a role of visible-light in this catalytic reactions.

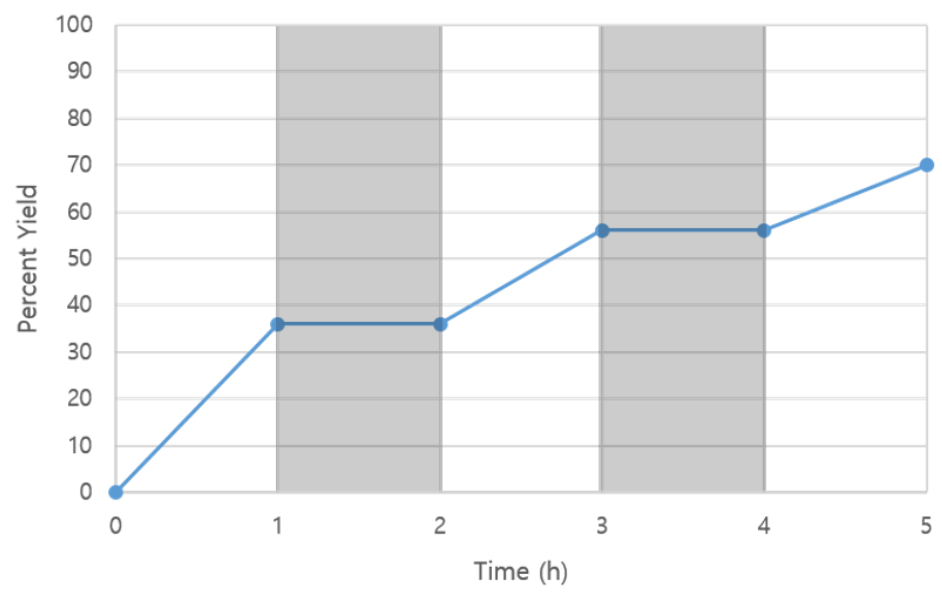

Figure S3 Light on/off experiment of 5a

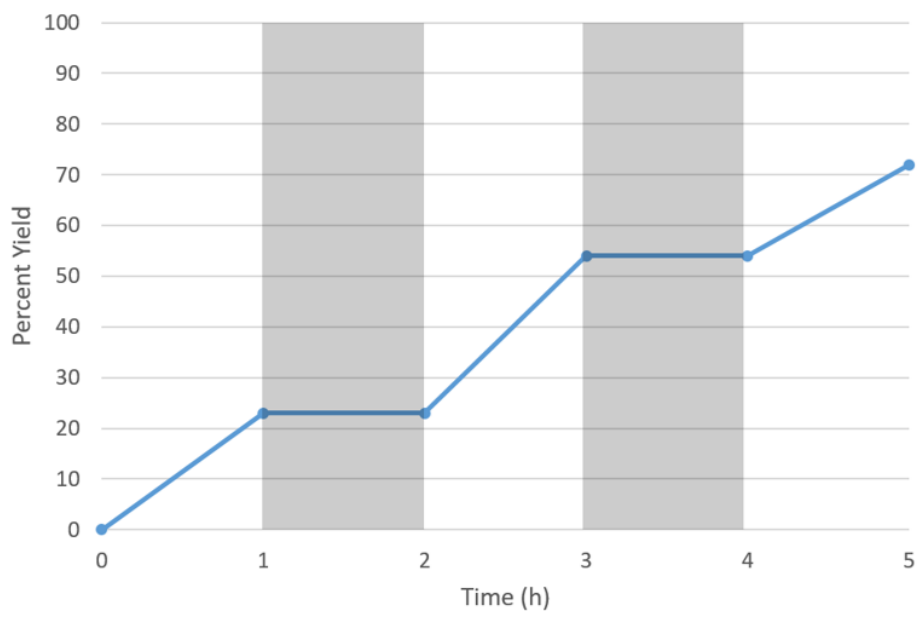

Figure S4 Light on/off experment of 6 a 


\section{Quantum Yield Analysis}

\section{Determination of the light intensity at $455 \mathrm{~nm}$}

Following a literature procedure of Yoon, ${ }^{7}$ the photon flux of the LED $\left(3 \mathrm{~W}, \lambda_{\max }=455 \mathrm{~nm}\right)$ was determined by standard ferrioxalate actinometry. ${ }^{8}$ A $0.15 \mathrm{M}$ ferrioxalate solution was prepared by dissolving $737 \mathrm{mg}$ of potassium ferrioxalate hydrate in $10 \mathrm{~mL}$ of $0.05 \mathrm{M} \mathrm{H}_{2} \mathrm{SO}_{4}$ aqueous solution. A buffered solution of 1,10-phenanthroline was prepared by dissolving $25 \mathrm{mg}$ of phenanthroline and $5.63 \mathrm{~g}$ of sodium acetate in $25 \mathrm{~mL}$ of $0.5 \mathrm{M} \mathrm{H}_{2} \mathrm{SO}_{4}$ aqueous solution. Both solutions were stored in the dark. To determine the photon flux of the LED, $1.0 \mathrm{~mL}$ of the ferrioxalate solution was placed in a $8.0 \mathrm{~mL}$ vial and irradiated for $60 \mathrm{~s}$ at $\lambda_{\max }=455 \mathrm{~nm}$ (see Figure 4 for the emission spectrum of the LED used). After irradiation, the phenanthroline solution $(175 \mu \mathrm{L})$ was added to the vial and the mixture was stirred for $1 \mathrm{~h}$ in the dark to allow the ferrous ions to completely coordinate to the phenanthroline. The solution was transferred to a quartz cuvette and the absorption of the solution was measured at $510 \mathrm{~nm}$. A non-irradiated sample was also prepared and the absorption at $510 \mathrm{~nm}$ was measured. Each sample preparation and measurement was repeated two more times. The average of the absorption of the irradiated and non-irradiated samples was determined and used to calculate the conversion applying eq. 1.

$$
\operatorname{mol~Fe} e^{2+}=\frac{V \cdot \Delta \mathrm{A}(510 \mathrm{~nm})}{\mathrm{l} \cdot \varepsilon}
$$

where $\mathrm{V}$ is the total volume $(0.001175 \mathrm{~L})$ of the solution after addition of phenanthroline, $\Delta \mathrm{A}$ is the difference in absorption at $510 \mathrm{~nm}$ between the irradiated and non-irradiated solutions, 1 is the path length $(0.10 \mathrm{~cm})$, and $\varepsilon$ is the molar absorptivity at $510 \mathrm{~nm}\left(11,100 \mathrm{~L} \mathrm{~mol}^{-1} \mathrm{~cm}^{-1}\right){ }^{8 \mathrm{a}}$ The photon flux can be calculated using eq. 2 .

$$
\text { Photon flux }=\frac{\mathrm{mol} \mathrm{Fe}^{2+}}{\Phi \cdot t \cdot f}
$$

Where $\Phi$ is the quantum yield for the ferrioxalate actinometer $(0.93$ for a $0.15 \mathrm{M}$ solution at $\lambda=455 \mathrm{~nm}){ }^{8 \mathrm{~d}} \mathrm{t}$ is the irradiation time $(60 \mathrm{~s})$, and $\mathrm{f}$ is the fraction of light absorbed at $\lambda=455 \mathrm{~nm}(0.96) .{ }^{8 \mathrm{~d}}$ The photon flux was calculated (average of three experiments) to be $4.54 \cdot 10^{-9}$ einsteins $\mathrm{s}^{-1}$.

\section{Determination of the reaction quantum yield}

To a catalyst $3 \mathbf{c}$ solution prepared as described above in $1.0 \mathrm{~mL}$ of dichloromethane was successively added [ $\left.\operatorname{Ir}(\mathrm{dtbbpy})(\mathrm{ppy})_{2}\right]\left[\mathrm{BF}_{4}\right] 4$ (0.0025 mmol, 0.010 equiv.), aldehyde compound $\mathbf{1}$ (0.38 mmol, 1.5 equiv.) and $\alpha$-silyl amine compound 2 (0.25 mmol, 1.0 equiv.) at room temperature. The solution was stirred and irradiated at $\lambda_{\max }=455 \mathrm{~nm}(\mathrm{see}$ Figure 4 for an emission spectrum of the LED used) for $20 \mathrm{~min}$. After irradiation, the reaction mixture was quenched with $175 \mu \mathrm{L}$ of distilled water and stirred for $1 \mathrm{~min}$. The combined extract was dried over anhydrous $\mathrm{Na}_{2} \mathrm{SO}_{4}$, filtered and concentrated in vacuo. The yield was determined by NMR analysis using dimethyl terephthalate as an internal standard and the value was determined to be $58 \%\left(1.47 \cdot 10^{-4} \mathrm{~mol}\right)$.

$$
\Phi=\frac{\mathrm{n} \text { (product) }}{\text { Photon flux } \cdot \mathrm{t} \cdot \mathrm{f}_{\mathrm{R}}}
$$

The reaction quantum yield $(\Phi)$ was calculated using eq. 3 where the photon flux is $4.54 \cdot 10^{-9}$ einsteins s${ }^{-1}$ (determined by actinometry as described above), $t$ is the reaction time $(1200 \mathrm{~s})$ and $f_{R}$ is the fraction of incident light absorbed by the reaction mixture, determined using eq. 4 where $\mathrm{A}(455 \mathrm{~nm})$ is the absorption of the reaction mixture at $455 \mathrm{~nm}$.

$$
\mathrm{f}_{\mathrm{R}}=1-10^{-\mathrm{A}(455 \mathrm{~nm})}
$$

An absorption spectrum of the reaction mixture gave an absorbance value of 1.0 at $455 \mathrm{~nm}$, indicating that the fraction of absorbed light $\left(\mathrm{f}_{\mathrm{R}}\right)$ is 0.90

The reaction quantum yield $(\Phi)$ was thus determined to be $\Phi=30$. 


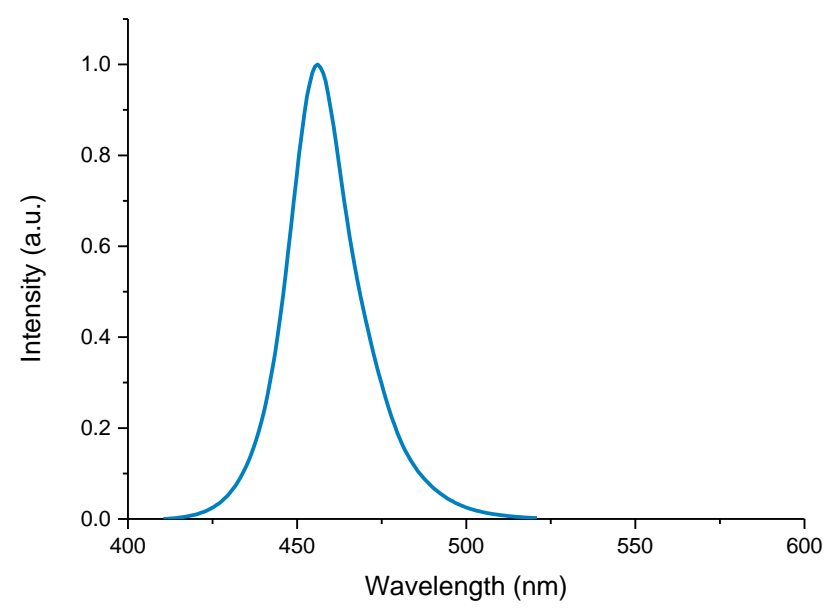

Figure S5 Emmision spectrum of 3W LED 


\section{Chemical Transformations of $\beta$-Amino Alcohols}

\subsection{Chemical Transformation of 5 a to 8}<smiles>NC[C@H](O)C1=CCCCC1</smiles>

$5 a(92 \%$ ee $)$

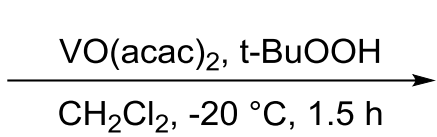<smiles>NC[C@H](O)[C@]12CCCC[C@H]1O2</smiles>

$71 \%$ yield, $92 \%$ ee (single diastereomer)

(S)-1-((1S,6S)-7-oxabicyclo[4.1.0]heptan-1-yl)-2-(diphenylamino)ethanol (8)<smiles>O[C@H]1O[C@H]1CNP</smiles>

To stirred solution of $5 \mathbf{a}(60 \mathrm{mg}, 0.20 \mathrm{mmol}, 1.0$ equiv.) in dichloromethane $(1.0 \mathrm{~mL})$ was added vanadyl acetylacetonate (1 $\mathrm{mg}, 0.0041 \mathrm{mmol}, 0.020$ equiv.) and $5.0 \mathrm{M}$ tert-butyl hydroperoxide solution in decane (57 $\mu \mathrm{L}, 0.29 \mathrm{mmol}, 1.4$ equiv.) at -20 ${ }^{\circ} \mathrm{C}$. The reaction mixture was stirred at $-20^{\circ} \mathrm{C}$ for $1.5 \mathrm{~h}$. After completion of the reaction, the reaction mixture was filtered through a pad of alumina. The filtrate was concentrated in vacuo. The residue was purified by column chromatography (gradient elution with 3-12\% ethyl acetate-hexane) to give (S)-1-((1S,6S)-7-oxabicyclo[4.1.0]heptan-1-yl)-2(diphenylamino)ethanol as white solid (45 mg, $71 \%$ ).

${ }^{1} \mathrm{H}$ NMR $\left(500 \mathrm{MHz}, \mathrm{CDCl}_{3}\right) \delta 7.27-7.24(\mathrm{~m}, 4 \mathrm{H}), 7.08(\mathrm{~d}, \mathrm{~J}=7.8 \mathrm{~Hz}, 4 \mathrm{H}), 6.97-6.94(\mathrm{~m}, 2 \mathrm{H}), 4.03(\mathrm{dd}, \mathrm{J}=14.5,2.6 \mathrm{~Hz}$, $1 \mathrm{H}), 3.80(\mathrm{dd}, \mathrm{J}=14.6,8.2 \mathrm{~Hz}, 1 \mathrm{H}), 3.75(\mathrm{dd}, \mathrm{J}=8.2,2.5 \mathrm{~Hz}, 1 \mathrm{H}), 3.19(\mathrm{~d}, \mathrm{~J}=2.4 \mathrm{~Hz}, 1 \mathrm{H}), 2.40(\mathrm{br} \mathrm{s}, 1 \mathrm{H}), 1.97-1.91$ (m, 2H), $1.77-1.68(\mathrm{~m}, 2 \mathrm{H}), 1.50-1.35(\mathrm{~m}, 2 \mathrm{H}), 1.26-1.16(\mathrm{~m}, 2 \mathrm{H})$

${ }^{13} \mathbf{C}$ NMR $\left(125 \mathrm{MHz}, \mathrm{CDCl}_{3}\right) \delta 148.5,129.4,121.9,121.6,71.2,60.7,56.7,55.1,24.6,24.5,20.2,19.4$ IR $v_{\max } 3447,3087,3057,3021,2933,1588,1496,1362,1298,1246,1215,1094,1029,989,872,845 \mathrm{~cm}^{-1}$.

LRMS (ESI): Calcd. for $\mathrm{C}_{20} \mathrm{H}_{23} \mathrm{NNaO}_{2}: \mathrm{m} / \mathrm{z} 332.16\left([\mathrm{M}+\mathrm{Na}]^{+}\right)$, found: $\mathrm{m} / \mathrm{z}(\%)=332.16\left([\mathrm{M}+\mathrm{Na}]^{+}\right)$

HPLC: $92 \%$ ee, IC, 2-propanol: $n$-Hexane=1:9, flow: $1.0 \mathrm{~mL} / \mathrm{min}, 220 \mathrm{~nm}, T_{\mathrm{R}}=6.42 \mathrm{~min}$ (minor) and $T_{\mathrm{R}}=7.30 \mathrm{~min}$ (major). $[\alpha]^{25}=+7.6\left(c=1.0, \mathrm{CHCl}_{3}\right)$. 


\subsection{Chemical Transformation of $5 b$ to 10}<smiles>COc1ccc(N(C[C@H](O)C2=CCCCC2)c2ccc(OC)cc2)cc1</smiles>

5b (93\% ee)

$$
\begin{aligned}
& \text { 1) } \mathrm{Bz}_{2} \mathrm{O}, \mathrm{Et}_{3} \mathrm{~N}, \mathrm{DMAP} \\
& \mathrm{CH}_{2} \mathrm{Cl}_{2}, \mathrm{RT}, 2 \mathrm{~h} \\
& \hline \text { 2) }\left(\mathrm{NH}_{4}\right)_{2} \mathrm{Ce}\left(\mathrm{NO}_{3}\right)_{6} \\
& \mathrm{H}_{2} \mathrm{O} / \mathrm{CH}_{3} \mathrm{CN}, 0^{\circ} \mathrm{C}, 2 \mathrm{~h}
\end{aligned}
$$

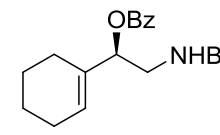

9

$50 \%$ yield, $93 \%$ ee

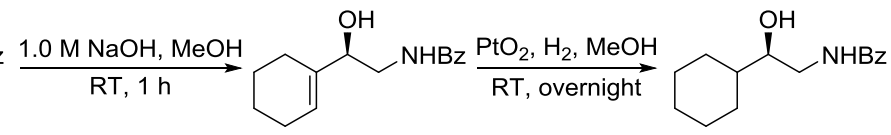

$9^{\prime}$

$99 \%$ yield, $93 \%$ ee
10 $89 \%$ yield, $93 \%$ ee

\section{(R)-2-benzamido-1-cyclohexenylethyl benzoate (9)}<smiles>O=C(NCC(OC(=O)c1ccccc1)C1=CCCCC1)c1ccccc1</smiles>

To a stirred solution of $\mathbf{5 b}(190 \mathrm{mg}, 0.54 \mathrm{mmol}, 1.0$ equiv.) in dichloromethane (3.0 mL) was added benzoic anhydride (290 $\mathrm{mg}, 1.3 \mathrm{mmol}, 2.5$ equiv.), 4-dimethylaminopyridine ( $13 \mathrm{mg}, 0.11 \mathrm{mmol}, 0.20$ equiv.), and triethylamine ( $0.24 \mathrm{~mL}, 1.7$ mmol, 3.1 equiv.). The reaction mixture was stirred at room temperature for $2 \mathrm{~h}$. After completion of the reaction, the reaction mixture was quenched with distilled water $(5.0 \mathrm{~mL})$ and extracted with dichloromethane $(3 \times 5.0 \mathrm{~mL})$. The combined extract was dried over $\mathrm{Na}_{2} \mathrm{SO}_{4}$, filtered and concentrated in vacuo. The residue was dissolved in acetonitrile (3.0 $\mathrm{mL})$ and ceric ammonium nitrate $(1.5 \mathrm{~g}, 2.7 \mathrm{mmol}, 5.0$ equiv.) in distilled water $(3.0 \mathrm{~mL})$ was added to the reaction mixture at $0{ }^{\circ} \mathrm{C}$ slowly. After $2 \mathrm{~h}$, the reaction mixture was quenched with saturated $\mathrm{NaHCO}_{3}$ and filtered through a pad of Celite. After removing the solvent in vacuo, distilled water $(5.0 \mathrm{~mL})$ and ethyl acetate $(5.0 \mathrm{~mL})$ were added. The aqueous layer was extracted with ethyl acetate $(3 \times 5.0 \mathrm{~mL})$. The combined extract was dried over $\mathrm{Na}_{2} \mathrm{SO}_{4}$, filtered and concentrated in vacuo. The residue was purified by column chromatography (gradient elution with 5-20\% ethyl acetate-hexane) to give $(R)-2$ benzamido-1-cyclohexenylethyl benzoate as colorless liquid (94 mg, 50\%).

${ }^{1} \mathbf{H}$ NMR $\left(500 \mathrm{MHz}, \mathrm{CDCl}_{3}\right) \delta 8.06(\mathrm{~d}, \mathrm{~J}=7.3 \mathrm{~Hz}, 2 \mathrm{H}), 7.72(\mathrm{~d}, \mathrm{~J}=7.2 \mathrm{~Hz}, 2 \mathrm{H}), 7.59-7.56(\mathrm{~m}, 1 \mathrm{H}), 7.49-7.39(\mathrm{~m}, 5 \mathrm{H}), 6.53$ (br s, 1H), 5.90 (br s, 1H), $5.56(\mathrm{dd}, \mathrm{J}=7.7,3.8 \mathrm{~Hz}, 1 \mathrm{H}), 3.89-3.77$ (m, 2H), 2.15 (br s, 2H), 2.06 (br s, 2H), $1.72-1.56(\mathrm{~m}$, $4 \mathrm{H})$

${ }^{13}$ C NMR (125MHz, $\left.\mathrm{CDCl}_{3}\right) \delta 167.7,166.6,134.6,134.0,133.4,131.6,130.2,129.9,128.7,128.6,127.0,126.3,42.9,29.8$, $25.1,24.8,22.5,22.3$

IR $v_{\max } 3416,3319,3083,3060,2928,1719,1650,1603,1580,1488,1315,1269,1115,1026,924,843 \mathrm{~cm}^{-1}$.

HRMS (ESI, positive mode): Calcd. for $\mathrm{C}_{22} \mathrm{H}_{23} \mathrm{NO}_{3} \mathrm{Na}: \mathrm{m} / \mathrm{z} 372.1570\left([\mathrm{M}+\mathrm{Na}]^{+}\right)$, found: $\mathrm{m} / \mathrm{z} 372.1572\left([\mathrm{M}+\mathrm{Na}]^{+}\right)$

HPLC: $93 \%$ ee, OD-H, 2-propanol: $n$-Hexane=1:9, flow: $1.0 \mathrm{~mL} / \mathrm{min}, 220 \mathrm{~nm}, T_{\mathrm{R}}=9.18 \mathrm{~min}$ (minor) and $T_{\mathrm{R}}=10.59 \mathrm{~min}$ (major).

$[\alpha]^{25} \mathrm{D}=-6.0\left(c=0.60, \mathrm{CHCl}_{3}\right)$.

\section{(R)-N-(2-cyclohexenyl-2-hydroxyethyl)benzamide (9')}<smiles>O=C(NC[C@H](O)C1=CCCCC1)c1ccccc1</smiles> 
To a stirred solution of 9 (94 mg, $0.27 \mathrm{mmol}, 1.0$ equiv.) in methanol (1.0 mL) was added 1.0 $\mathrm{M} \mathrm{NaOH}(0.30 \mathrm{~mL}, 0.30$ mmol, 1.1 equiv.) and the reaction mixture was stirred at room temperature for overnight. After completion of the reaction, the reaction mixture was quenched with saturated $\mathrm{NH}_{4} \mathrm{Cl}(5.0 \mathrm{~mL})$ and extracted with ethyl acetate $(3 \times 5.0 \mathrm{~mL})$. The combined extract was dried over $\mathrm{Na}_{2} \mathrm{SO}_{4}$, filtered and concentrated in vacuo. The residue was purified by column chromatography (gradient elution with $17-50 \%$ ethyl acetate-hexane) to give $(R)-N$-(2-cyclohexenyl-2hydroxyethyl)benzamide as white solid $(66 \mathrm{mg}, 99 \%)$

${ }^{1}$ H NMR $\left(500 \mathrm{MHz}, \mathrm{CDCl}_{3}\right) \delta 7.77-7.76(\mathrm{~m}, 2 \mathrm{H}), 7.50-7.48(\mathrm{~m}, 1 \mathrm{H}), 7.44-7.41(\mathrm{~m}, 2 \mathrm{H}), 6.55(\mathrm{br} \mathrm{s}, 1 \mathrm{H}), 5.79(\mathrm{~d}$, $\mathrm{J}=1.2 \mathrm{~Hz}, 1 \mathrm{H}), 4.18(\mathrm{dd}, \mathrm{J}=7.7,2.6 \mathrm{~Hz}, 1 \mathrm{H}), 3.74(\mathrm{ddd}, \mathrm{J}=13.8,6.6,3.4 \mathrm{~Hz}, 1 \mathrm{H}), 3.42$ (ddd, J=13.7, 8.0, 4.9Hz, 1H), 2.45 (br s, $1 \mathrm{H}), 2.12-2.09(\mathrm{~m}, 1 \mathrm{H}), 2.05-2.03(\mathrm{~m}, 2 \mathrm{H}), 1.97-1.93(\mathrm{~m}, 1 \mathrm{H}), 1.70-1.54(\mathrm{~m}, 4 \mathrm{H})$

${ }^{13}$ C NMR $\left(125 \mathrm{MHz}, \mathrm{CDCl}_{3}\right) \delta 168.4,137.8,134.6,131.7,128.7,127.1,123.8,75.3,44.6,25.0,24.8,22.7,22.6$

IR $v_{\max } 3463,3018,2930,2897,1640,1604,1578,1489,1437,1313,1216,1156,1092,1027,921,844 \mathrm{~cm}^{-1}$.

HRMS (ESI, positive mode): Calcd. for $\mathrm{C}_{15} \mathrm{H}_{19} \mathrm{NO}_{2} \mathrm{Na}: \mathrm{m} / \mathrm{z} 268.1308\left([\mathrm{M}+\mathrm{Na}]^{+}\right)$, found: $\mathrm{m} / \mathrm{z} 268.1309\left([\mathrm{M}+\mathrm{Na}]^{+}\right)$ $[\alpha]^{25}=-21.6\left(c=1.0, \mathrm{CHCl}_{3}\right)$.

\section{(R)-N-(2-cyclohexyl-2-hydroxyethyl)benzamide (10)}<smiles>O=C(NC[C@H](O)C1CCCCC1)c1ccccc1</smiles>

To a stirred solution of 9' (60 mg, $0.24 \mathrm{mmol}, 1.0$ equiv.) in methanol (2.0 mL) was added platinum(IV) oxide (2.0 mg, 0.024 mmol, 0.10 equiv.). The reaction vessel was evacuated and purged with inert gas and subsequently evacuated and purged with hydrogen gas to ensure a complete hydrogen atmosphere. The reaction mixture was stirred at room temperature for overnight. After completion of the reaction, the reaction mixture was filtered through a pad of Celite and washed with methanol. After removing the solvent in vacuo, the residue was dissolved in a 1:1 mixture of ethyl acetate and distilled water. The residue was extracted with ethyl acetate $(3 \times 5.0 \mathrm{~mL})$. The combined extract was dried over $\mathrm{Na}_{2} \mathrm{SO}_{4}$, filtered and concentrated in vaсиo. The residue was purified by column chromatography (gradient elution with 17-50\% ethyl acetatehexane) to give $(R)-N$-(2-cyclohexyl-2-hydroxyethyl)benzamide as white solid (54 mg, 89\%).

${ }^{1}$ H NMR (500MHz, $\left.\mathrm{CDCl}_{3}\right) \delta 7.79-7.77(\mathrm{~m}, 2 \mathrm{H}), 7.51-7.48(\mathrm{~m}, 1 \mathrm{H}), 7.44-7.41$ (m, 2H), 6.65 (br s, $\left.1 \mathrm{H}\right), 3.77$ (ddd, $\mathrm{J}=13.9,6.7,2.8 \mathrm{~Hz}, 1 \mathrm{H}), 3.57-3.53(\mathrm{~m}, 1 \mathrm{H}), 3.36-3.31(\mathrm{~m}, 1 \mathrm{H}), 2.51(\mathrm{br} \mathrm{s}, 1 \mathrm{H}), 1.90-1.88(\mathrm{~m}, 1 \mathrm{H}), 1.80-1.73(\mathrm{~m}, 3 \mathrm{H})$, $1.70-1.65(\mathrm{~m}, 1 \mathrm{H}), 1.45-1.42(\mathrm{~m}, 1 \mathrm{H}), 1.26-1.19(\mathrm{~m}, 2 \mathrm{H}), 1.17-1.14(\mathrm{~m}, 1 \mathrm{H}), 1.11-1.03(\mathrm{~m}, 2 \mathrm{H})$

${ }^{13}$ C NMR $\left(125 \mathrm{MHz}, \mathrm{CDCl}_{3}\right) \delta 168.4,134.5,131.7,128.7,127.1,75.9,44.0,42.3,29.0,28.5,26.5,26.2,26.1$

IR $v_{\max } 3445,3015,2978,2922,2851,1638,1579,1549,1490,1449,1322,1262,1110,1065,967,769 \mathrm{~cm}^{-1}$.

HRMS (ESI, positive mode): Calcd. for $\mathrm{C}_{15} \mathrm{H}_{21} \mathrm{NO}_{2} \mathrm{Na}: \mathrm{m} / \mathrm{z} 270.1465\left([\mathrm{M}+\mathrm{Na}]^{+}\right)$, found: $\mathrm{m} / \mathrm{z} 270.1467\left([\mathrm{M}+\mathrm{Na}]^{+}\right)$ HPLC: $93 \%$ ee, OZ-H, 2-propanol: $n$-Hexane=2:8, flow: $1.0 \mathrm{~mL} / \mathrm{min}, 220 \mathrm{~nm}, T_{\mathrm{R}}=6.19 \min (\operatorname{minor})$ and $T_{\mathrm{R}}=8.10 \mathrm{~min}$ (major).

$[\alpha]^{25} \mathrm{D}=-17.8\left(c=1.0, \mathrm{CHCl}_{3}\right) ; \operatorname{lit}^{5}[\alpha]^{26} \mathrm{D}=-5.7\left(c=0.99, \mathrm{CHCl}_{3}\right)($ for $71 \%$ ee of $(R)-\mathbf{1 0})$. 


\subsection{Chemical Transformation of $6 \mathrm{k}$}

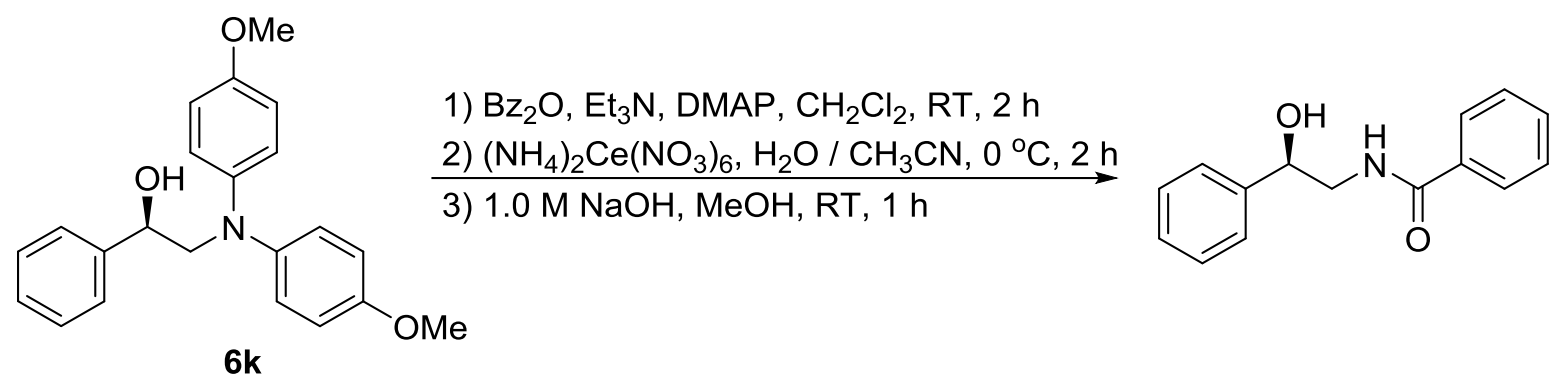

\section{(R)-N-(2-hydroxy-2-phenylethyl)benzamide}<smiles>O=C(NCC(O)c1ccccc1)c1ccccc1</smiles>

To a stirred solution of $6 \mathbf{k}(52 \mathrm{mg}, 0.15 \mathrm{mmol}, 1.0$ equiv.) in dichloromethane $(1.0 \mathrm{~mL})$ was added benzoic anhydride (84 $\mathrm{mg}, 0.37 \mathrm{mmol}, 2.5$ equiv.), 4-dimethylaminopyridine ( $4.0 \mathrm{mg}, 0.030 \mathrm{mmol}, 0.20$ equiv.), and triethylamine ( $0.064 \mathrm{~mL}, 0.46$ mmol, 3.1 equiv.). The reaction mixture was stirred at room temperature for $2 \mathrm{~h}$. After completion of the reaction, the reaction mixture was quenched with distilled water $(5.0 \mathrm{~mL})$ and extracted with dichloromethane $(3 \times 5.0 \mathrm{~mL})$. The combined extract was dried over $\mathrm{Na}_{2} \mathrm{SO}_{4}$, filtered and concentrated in vacuo. The residue was dissolved in acetonitrile $(0.50$ $\mathrm{mL})$ and ceric ammonium nitrate $(0.41 \mathrm{~g}, 0.74 \mathrm{mmol}, 5.0$ equiv. $)$ in distilled water $(0.50 \mathrm{~mL})$ was added to the reaction mixture at $0{ }^{\circ} \mathrm{C}$ slowly. After $2 \mathrm{~h}$, the reaction mixture was quenched with saturated $\mathrm{NaHCO}_{3}$ and filtered through a pad of Celite. After removing the solvent in vасиo, distilled water $(5.0 \mathrm{~mL})$ and ethyl acetate $(5.0 \mathrm{~mL})$ were added. The aqueous layer was extracted with ethyl acetate $(3 \times 5.0 \mathrm{~mL})$. The combined extract was dried over $\mathrm{Na}_{2} \mathrm{SO}_{4}$, filtered and concentrated in vacuo. The residue was diluted with methanol $(1.0 \mathrm{~mL})$ and added $1.0 \mathrm{M} \mathrm{NaOH}(0.16 \mathrm{~mL}, 0.16 \mathrm{mmol}, 1.1$ equiv. $)$ and the reaction mixture was stirred at room temperature for $1 \mathrm{~h}$. After completion of the reaction, the reaction mixture was quenched with saturated $\mathrm{NH}_{4} \mathrm{Cl}(5.0 \mathrm{~mL})$ and extracted with ethyl acetate $(3 \times 5.0 \mathrm{~mL})$. The combined extract was dried over $\mathrm{Na}_{2} \mathrm{SO}_{4}$, filtered and concentrated in vacuo. The residue was purified by column chromatography (gradient elution with 14$33 \%$ ethyl acetate-hexane) to give $(R)-N$-(2-hydroxy-2-phenylethyl)benzamide as white solid (22 $\mathrm{mg}, 61 \%$ ).

${ }^{1} \mathbf{H} \mathbf{N M R}\left(500 \mathrm{MHz}, \mathrm{CDCl}_{3}\right) \delta 7.74(\mathrm{~d}, \mathrm{~J}=7.3 \mathrm{~Hz}, 2 \mathrm{H}), 7.52-7.49(\mathrm{~m}, 1 \mathrm{H}), 7.43-7.40(\mathrm{~m}, 4 \mathrm{H}), 7.38-7.35(\mathrm{~m}, 2 \mathrm{H}), 7.32-$ 7.29 (m, 1H), 6.64 (br s, 1H), 4.96 (dd, J=8.2, 3.3Hz, 1H), 3.91 (ddd, J=13.9, 6.1, 3.4Hz, 1H), 3.52 (ddd, J=13.9, 8.3, 6.0Hz, $1 \mathrm{H}), 3.42$ (br s, 1H)

${ }^{13} \mathbf{C}$ NMR $\left(125 \mathrm{MHz}, \mathrm{CDCl}_{3}\right) \delta 168.8,141.9,134.2,13$ 1.9, 128.8, 128.7, 128.1, 127.1, 126.0, 73.9, 48.0

IR $v_{\max } 3593,3299,2913,2890,2837,1638,1577,1551,1492,1451,1262,1216,1038,1026,840 \mathrm{~cm}^{-1}$.

LRMS (ESI): Calcd. for $\mathrm{C}_{15} \mathrm{H}_{15} \mathrm{NNaO}_{2}: \mathrm{m} / \mathrm{z} 264.10\left([\mathrm{M}+\mathrm{Na}]^{+}\right)$, found: $\mathrm{m} / \mathrm{z}(\%)=264.10\left([\mathrm{M}+\mathrm{Na}]^{+}\right)$

HPLC: $70 \%$ ee, OJ-H, 2-propanol: $n$-Hexane=1:9, flow: $0.5 \mathrm{~mL} / \mathrm{min}, 220 \mathrm{~nm}, T_{\mathrm{R}}=19.46 \mathrm{~min}$ (major) and $T_{\mathrm{R}}=22.35 \mathrm{~min}$ (minor).

$[\alpha]^{25}{ }_{\mathrm{D}}=-55.3\left(c=0.50, \mathrm{CHCl}_{3}\right) ; \mathrm{lit}^{5}[\alpha]^{26}{ }_{\mathrm{D}}=-69.5\left(c=0.79, \mathrm{CHCl}_{3}\right)$ (for $97 \%$ ee of $(R)$-form). 
12. ${ }^{1} \mathrm{H}$ NMR and ${ }^{13} \mathrm{C}$ NMR Spectra

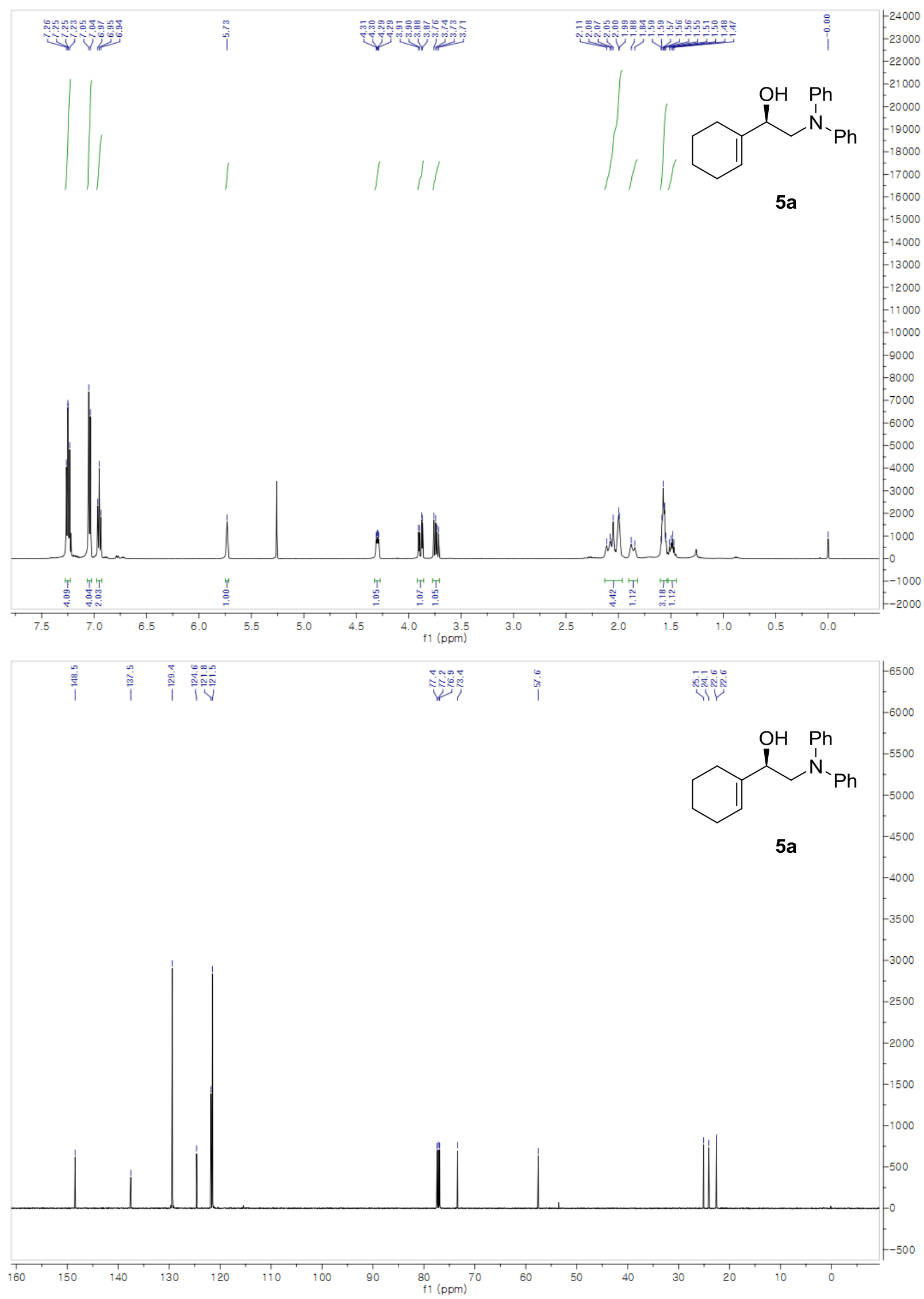




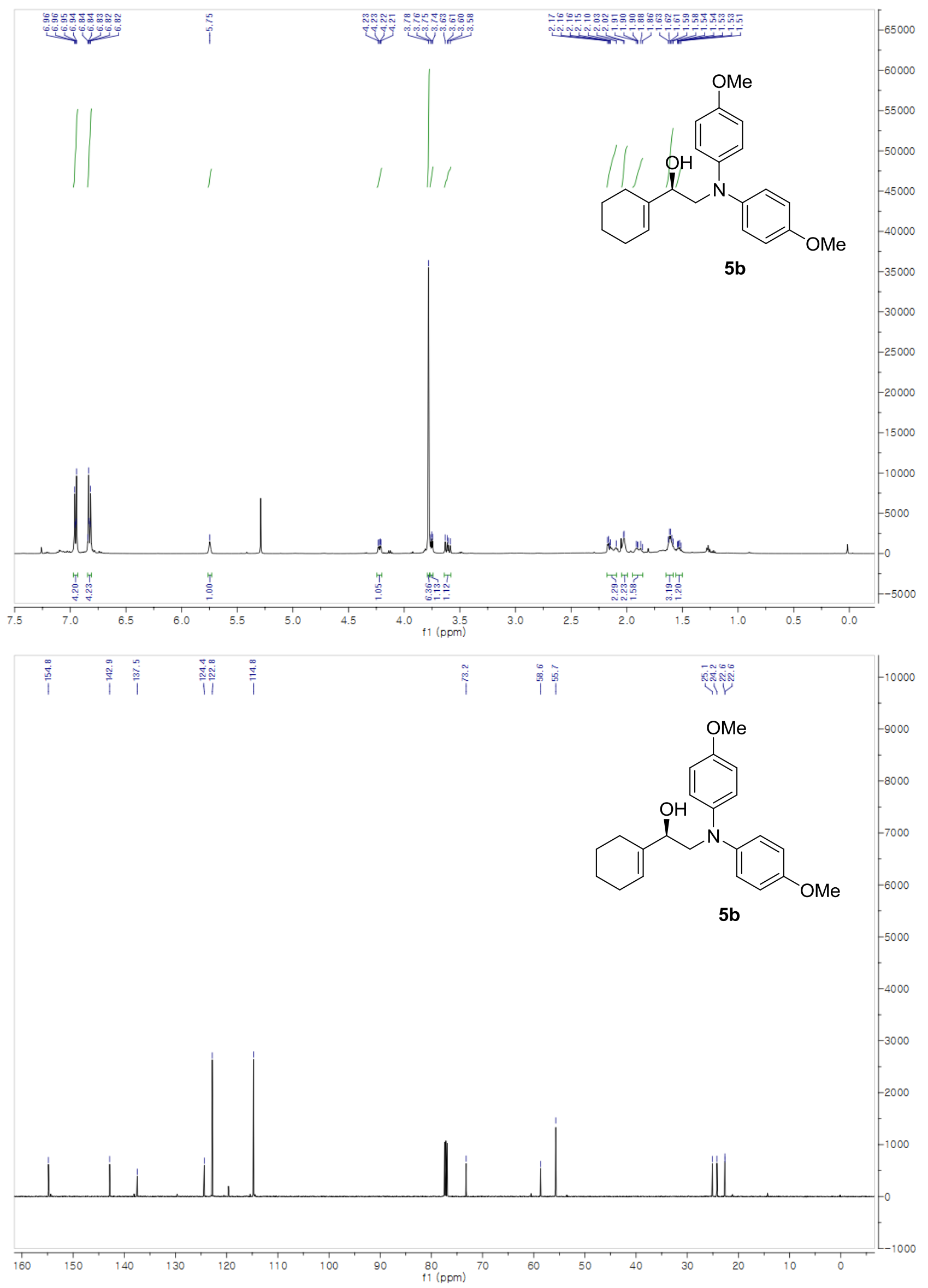



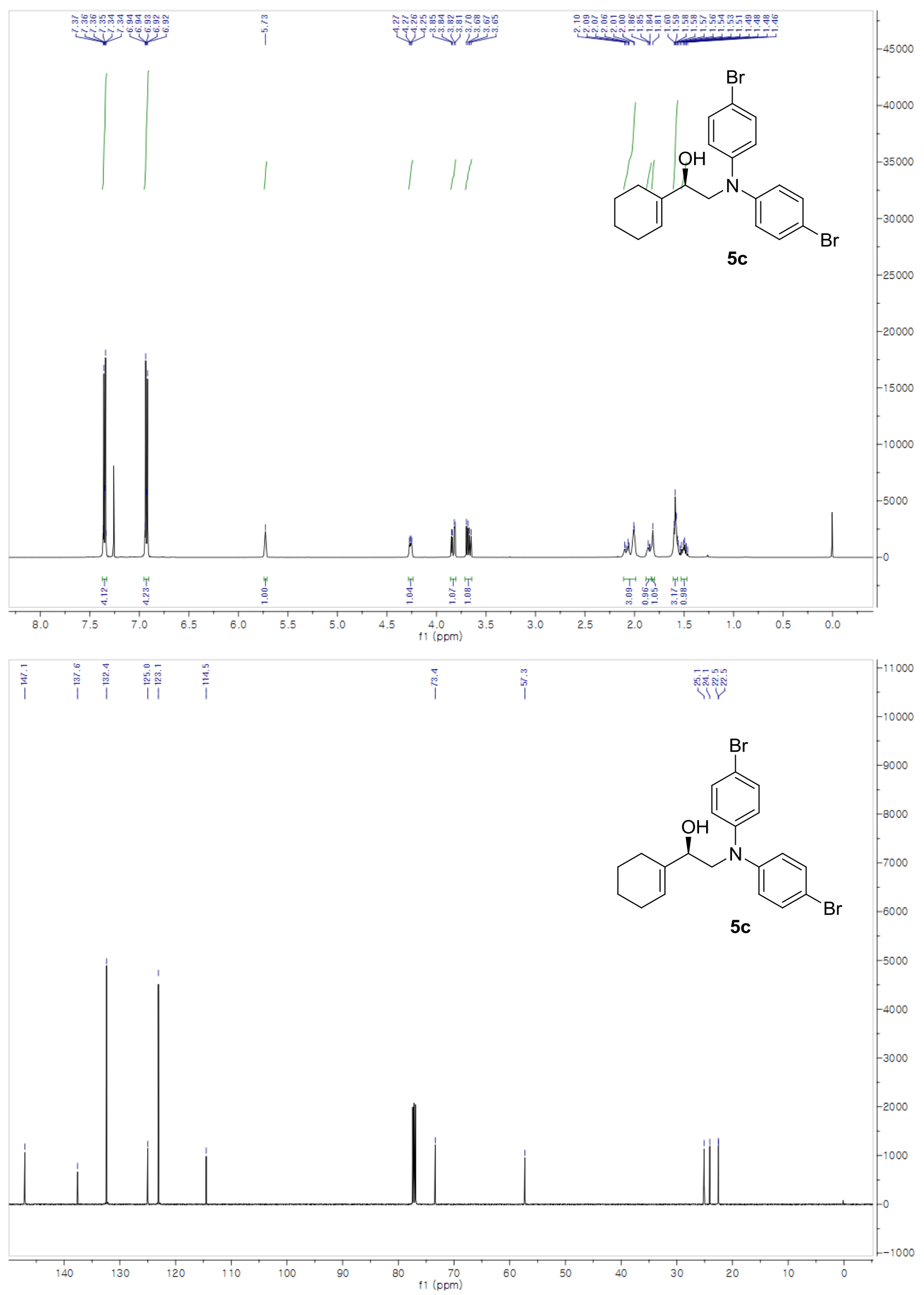

S39 


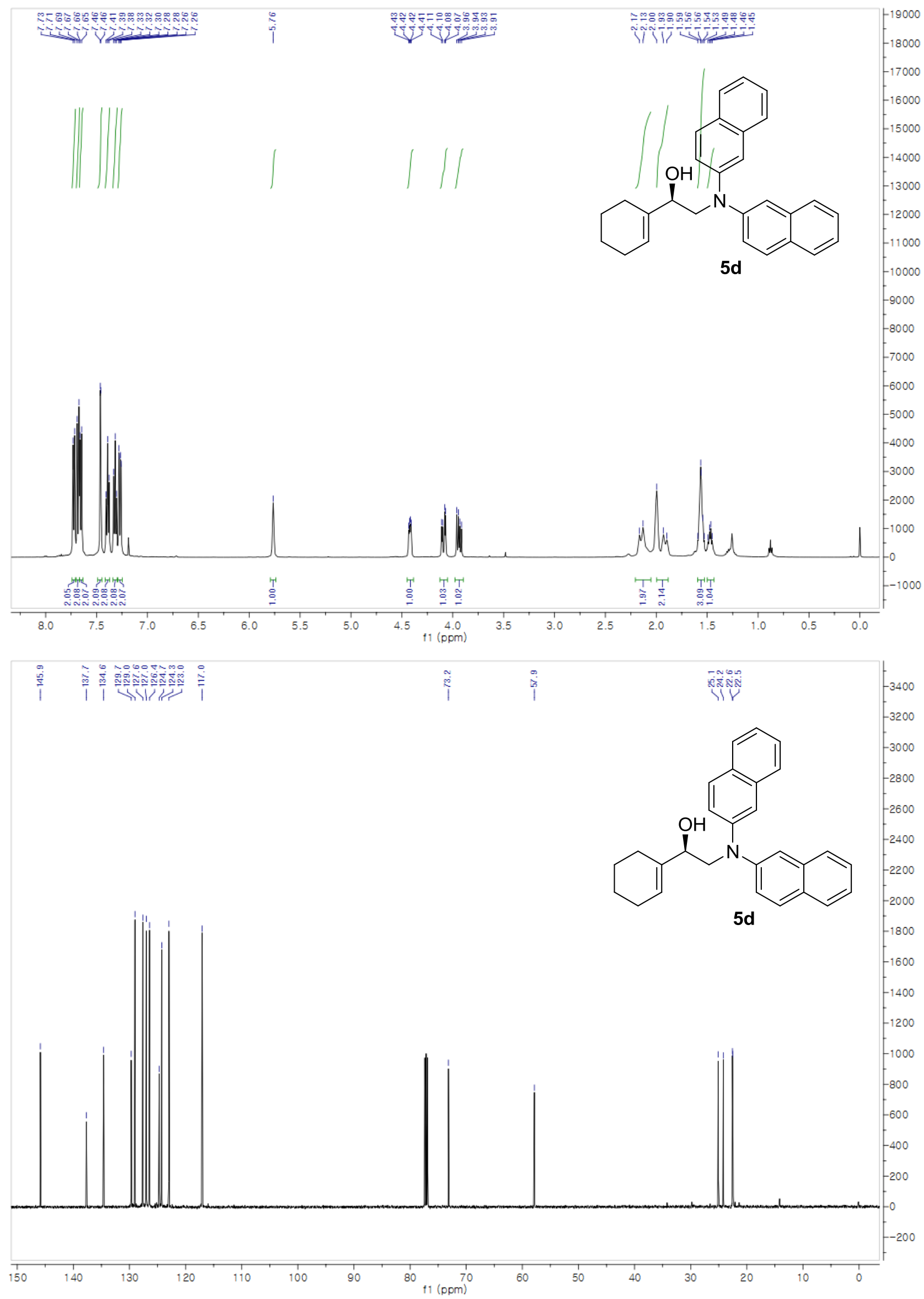




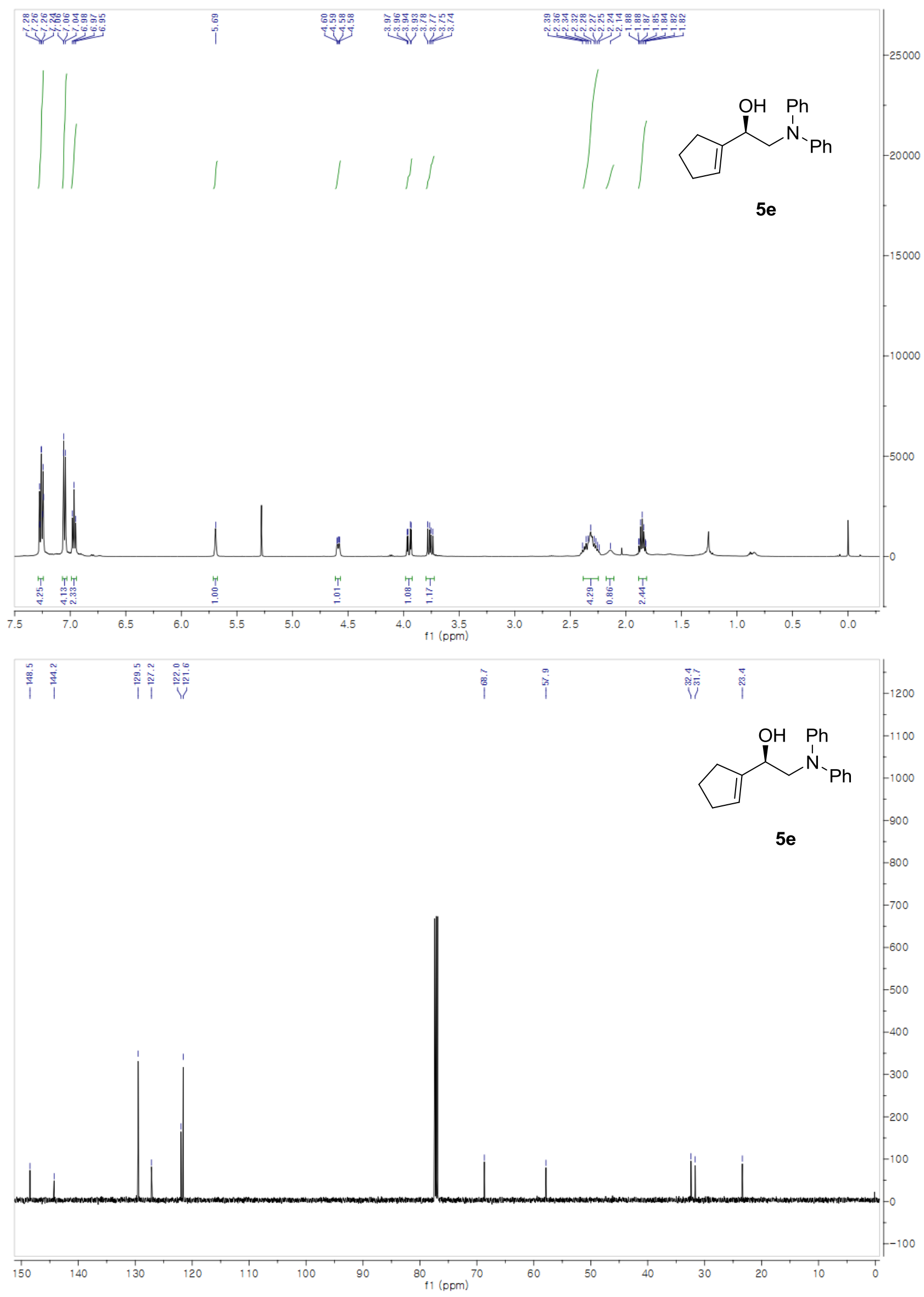




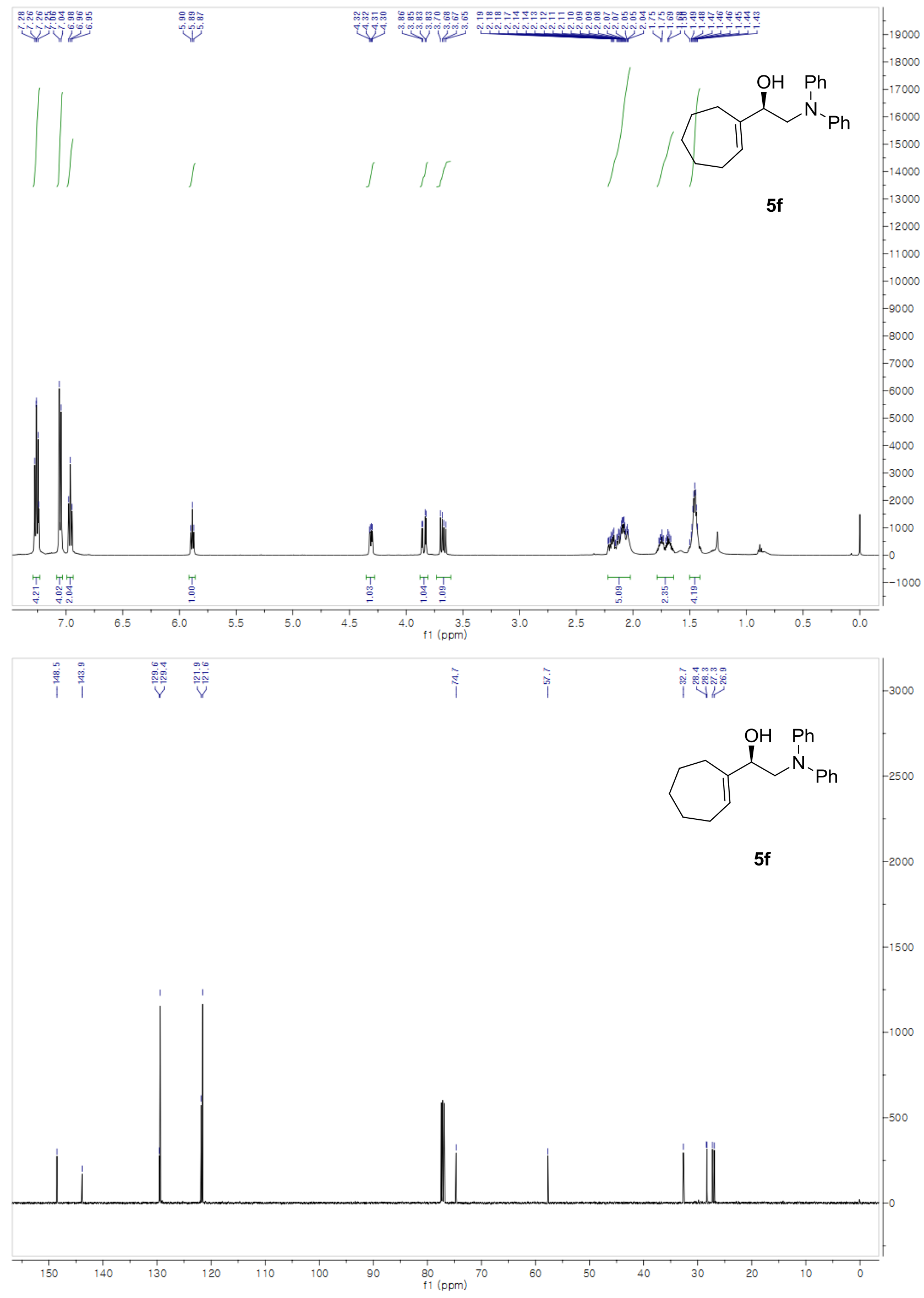




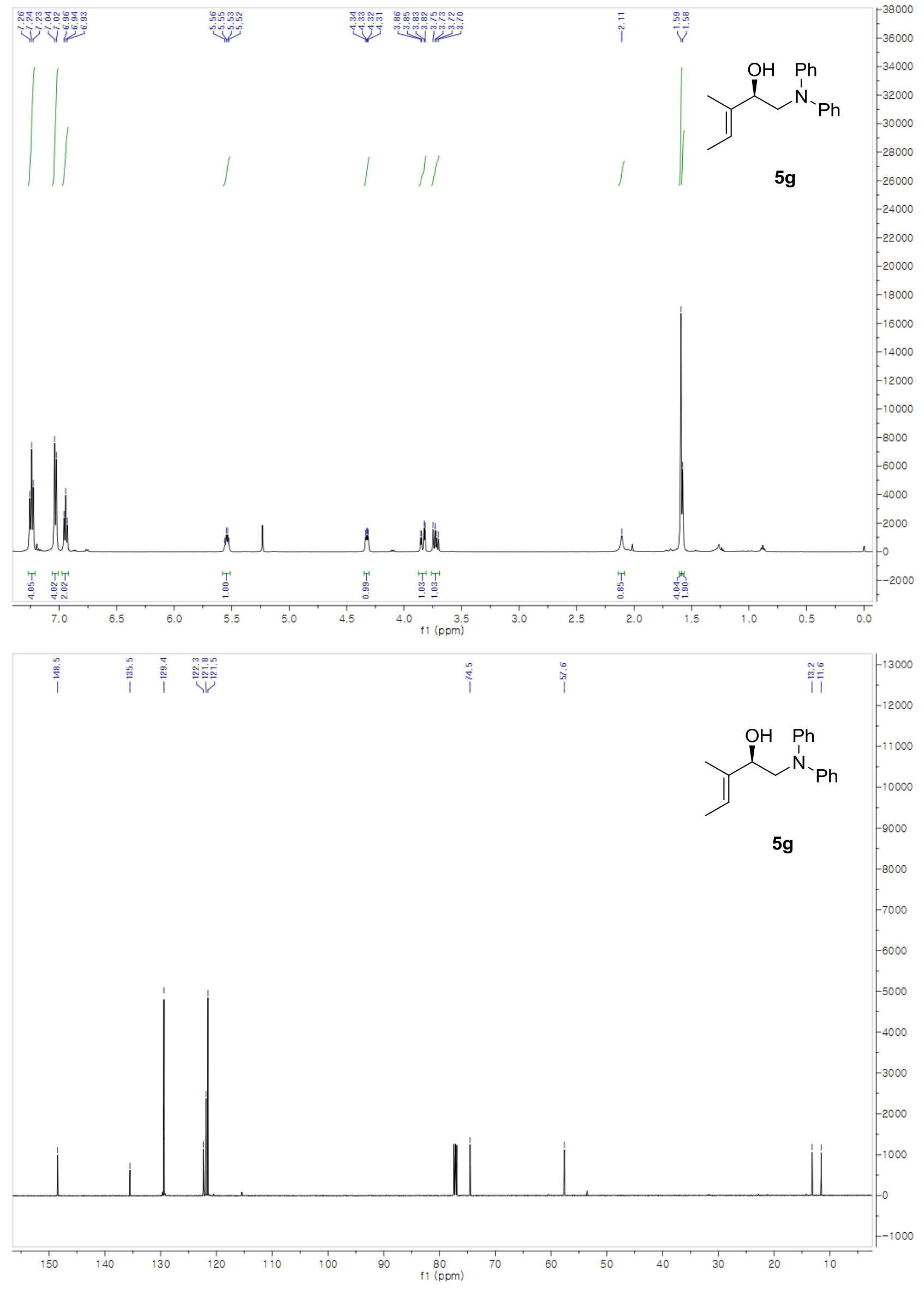




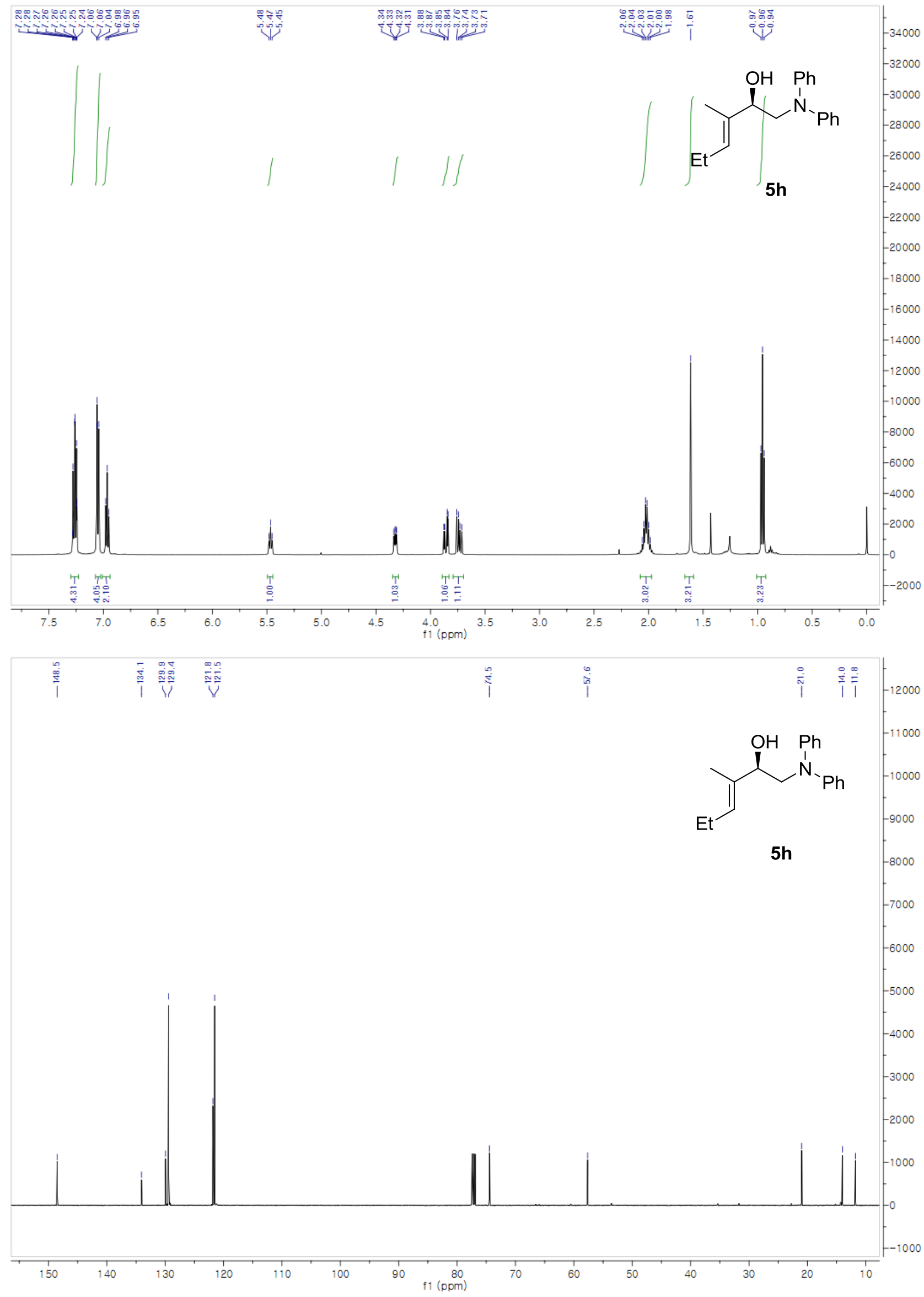




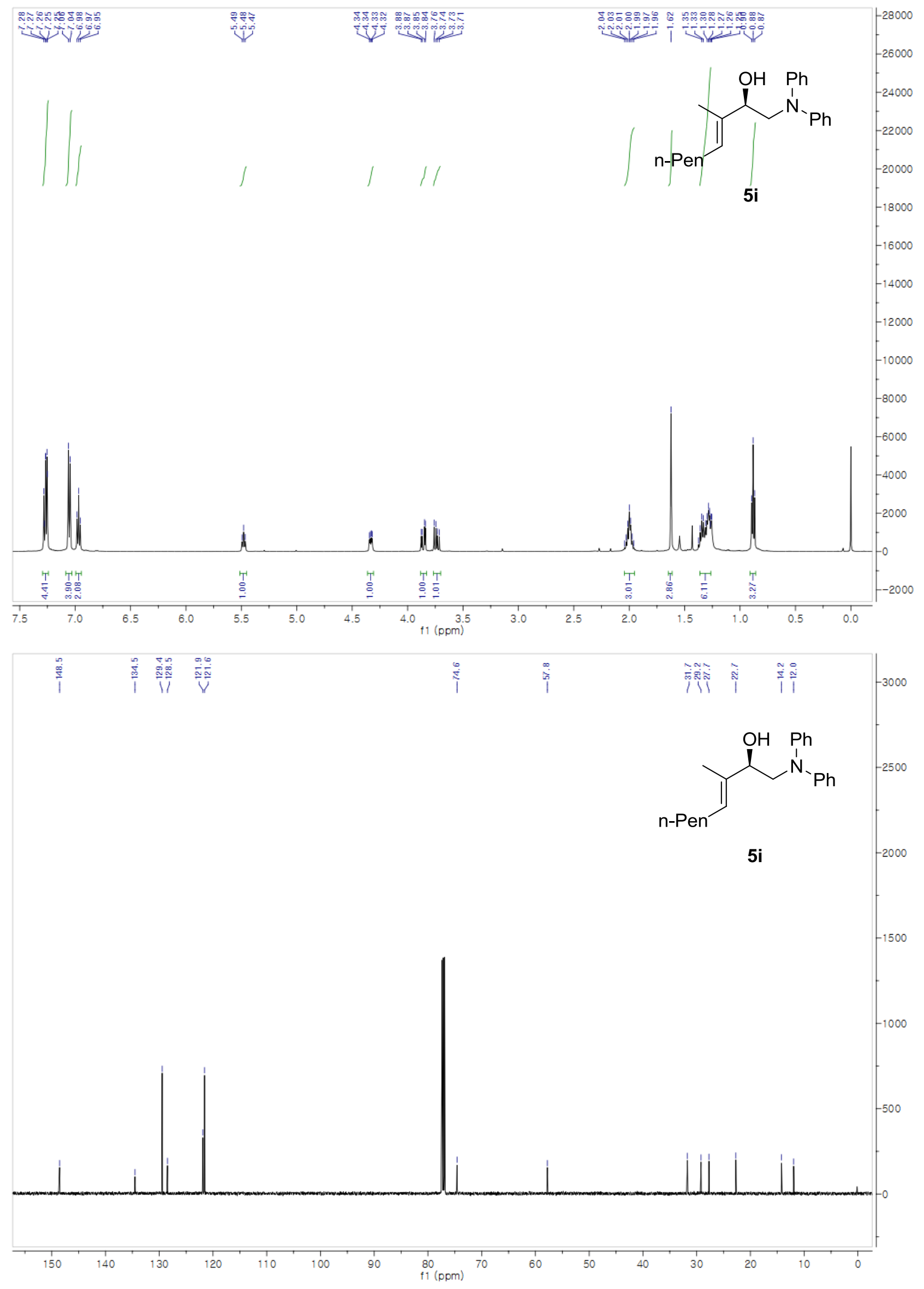




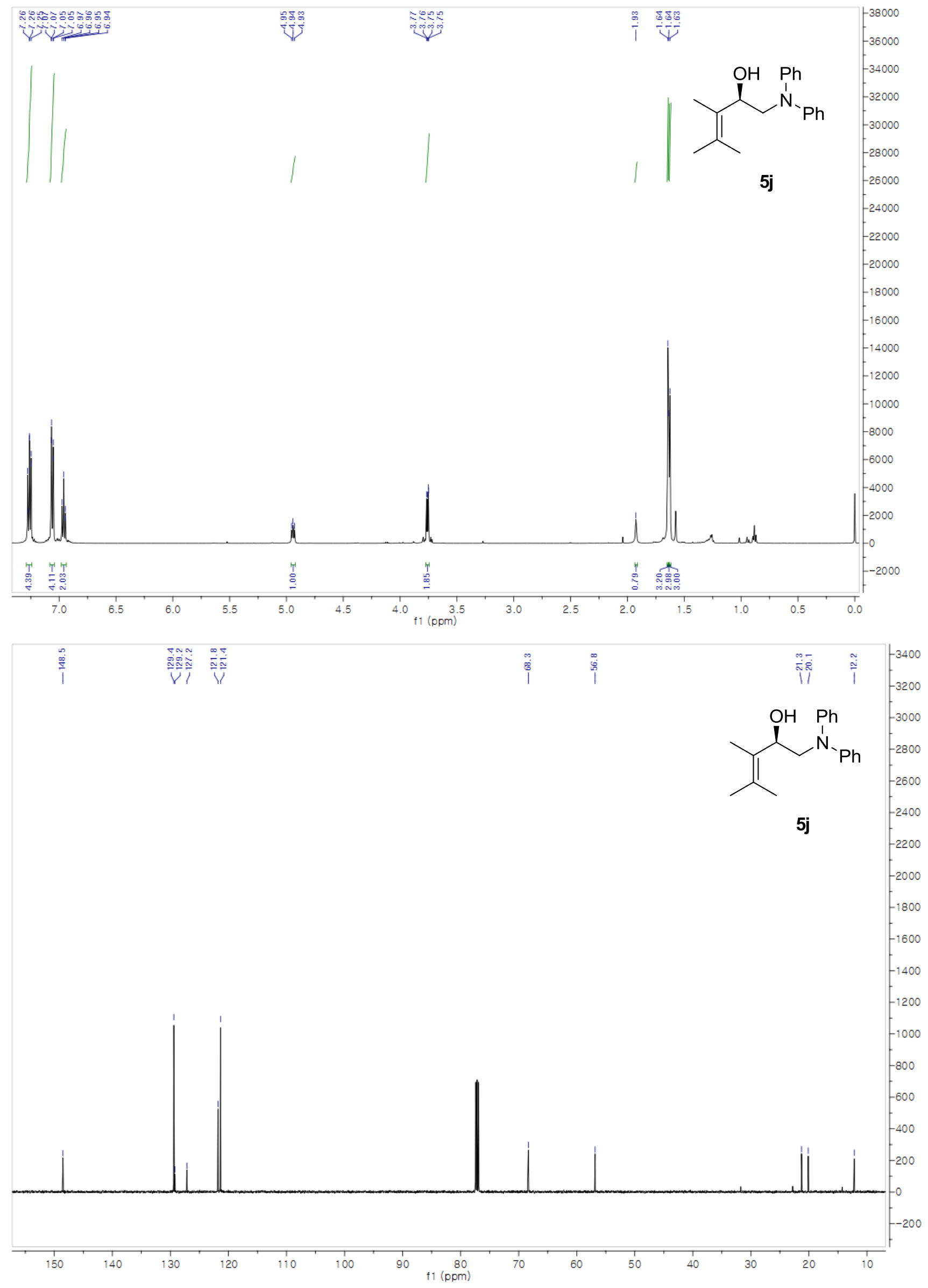




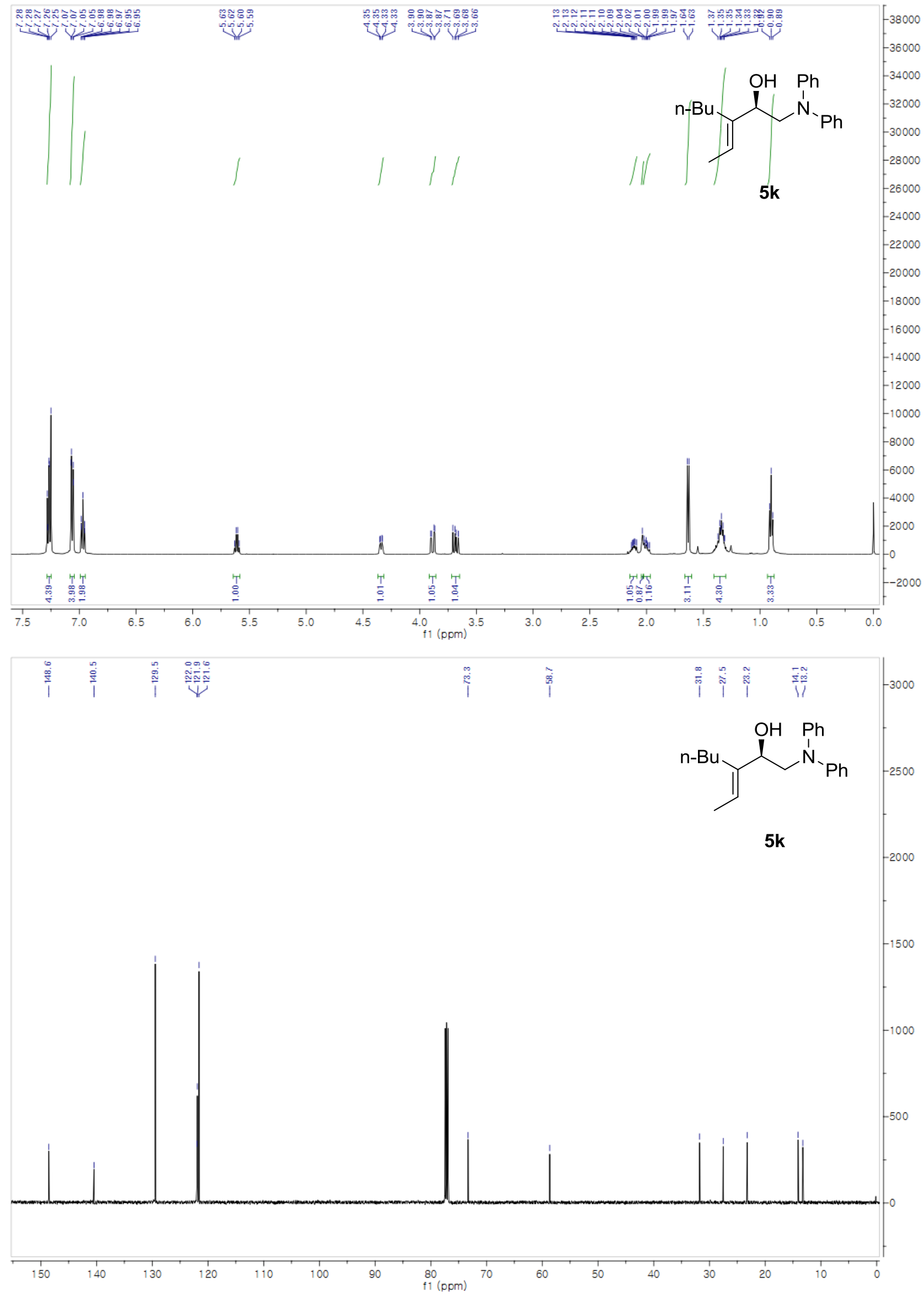




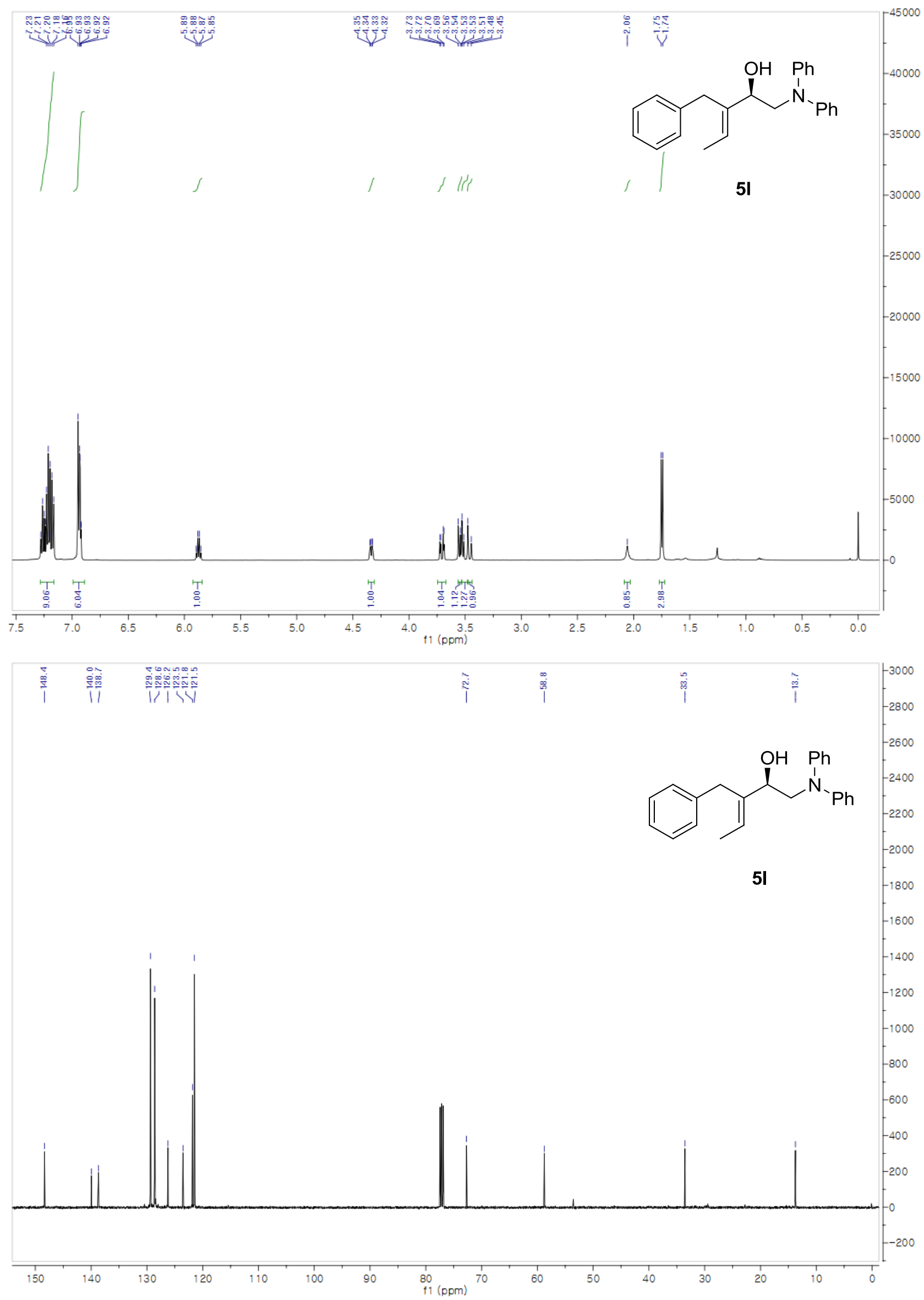




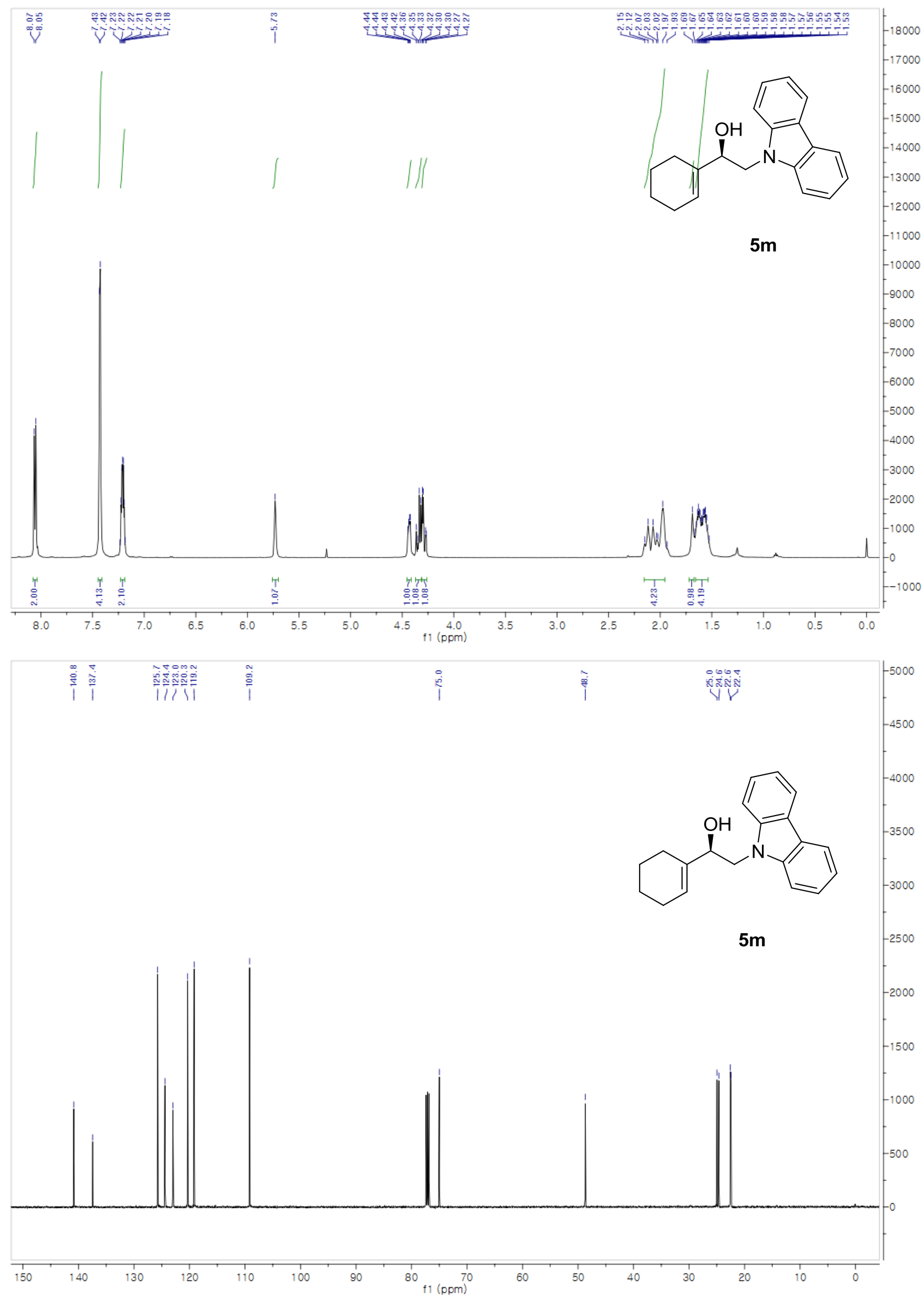




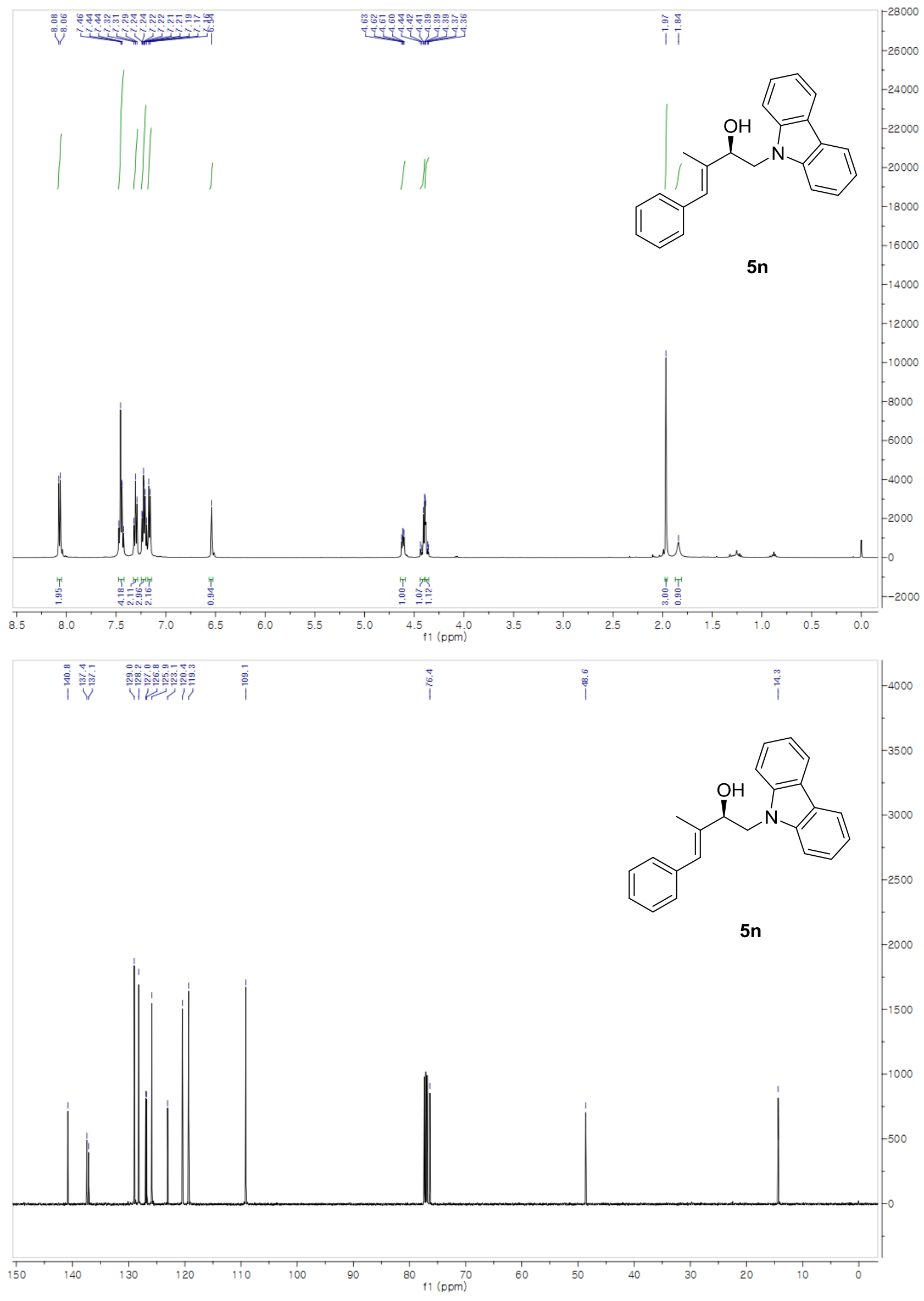




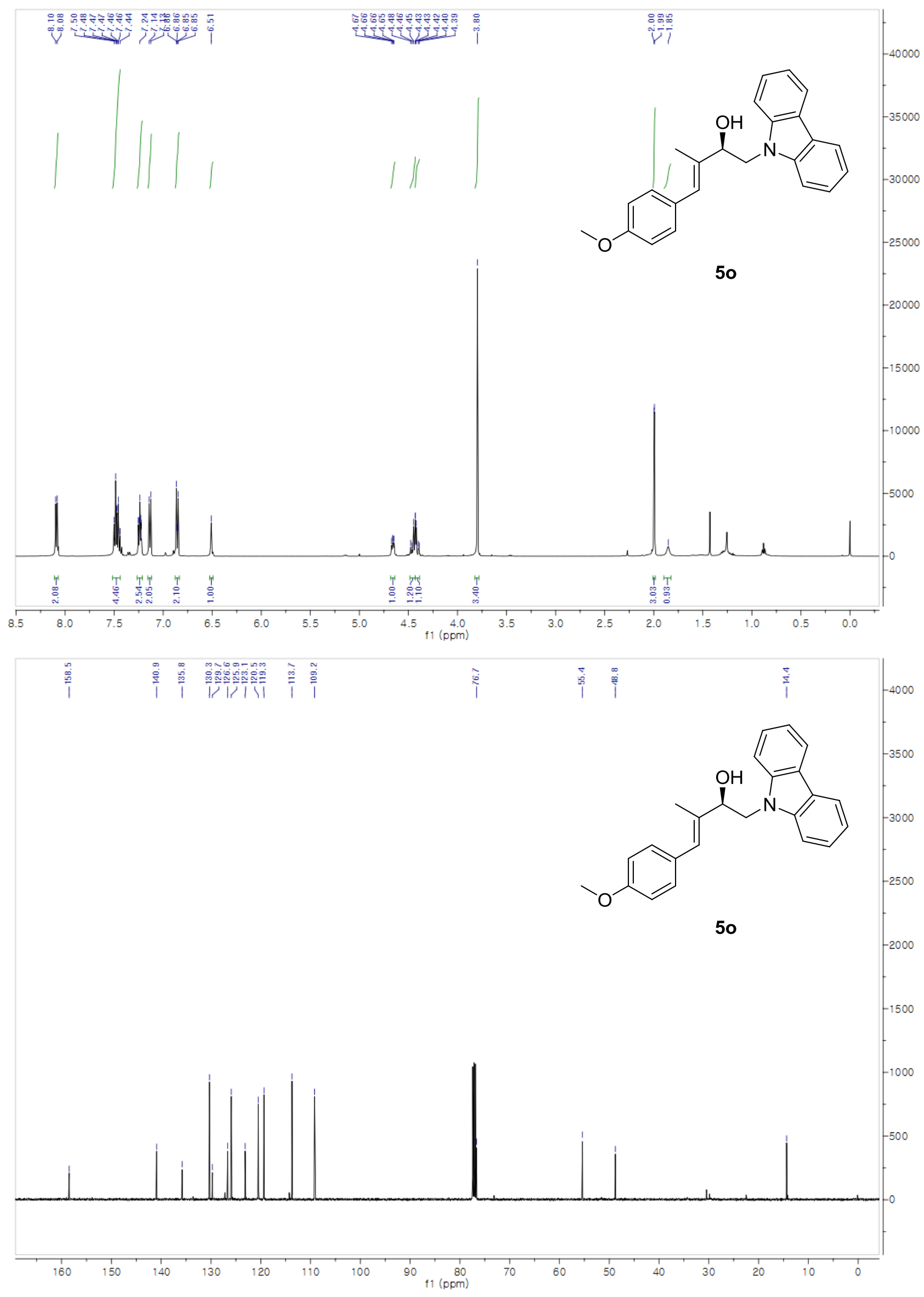




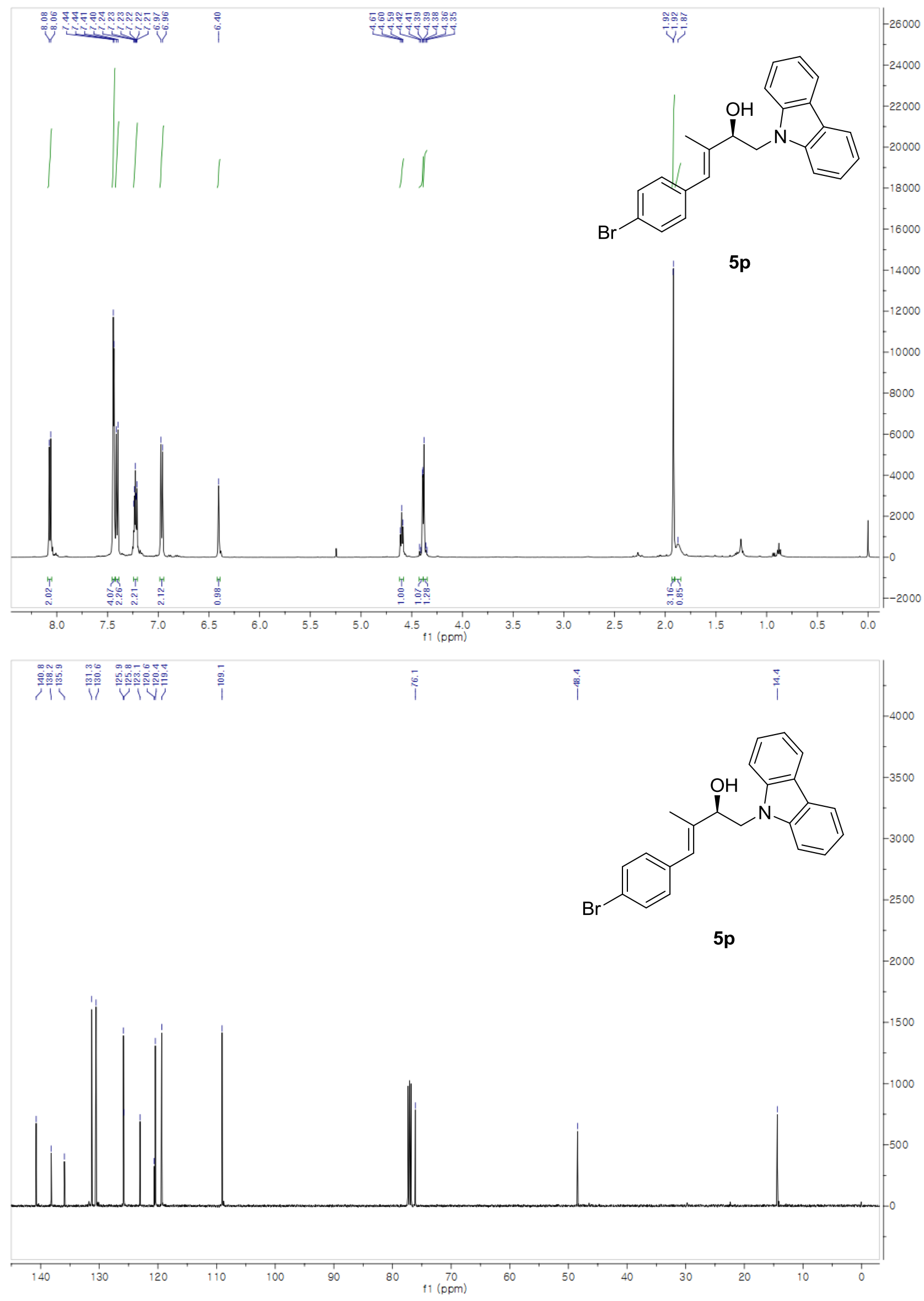




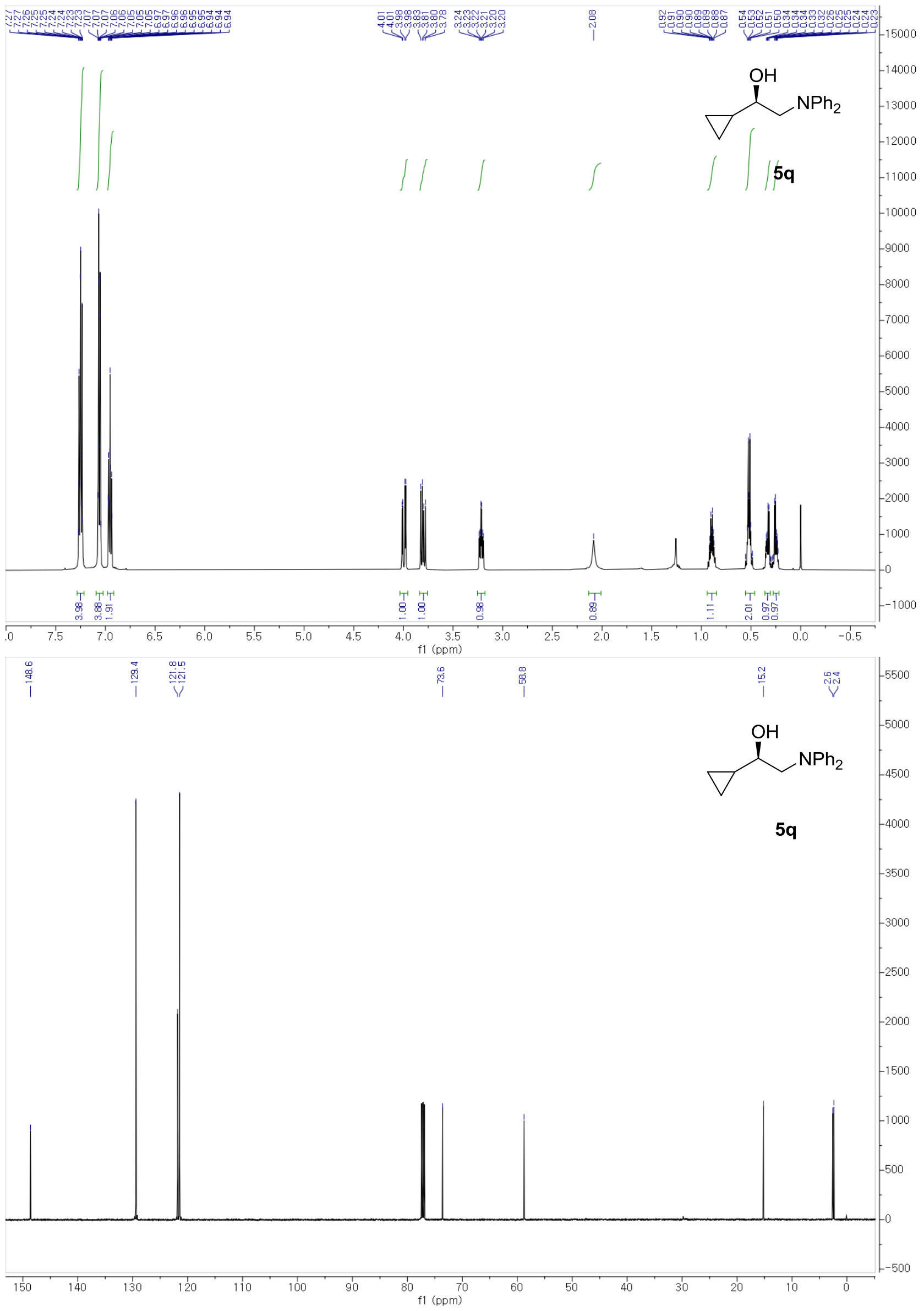




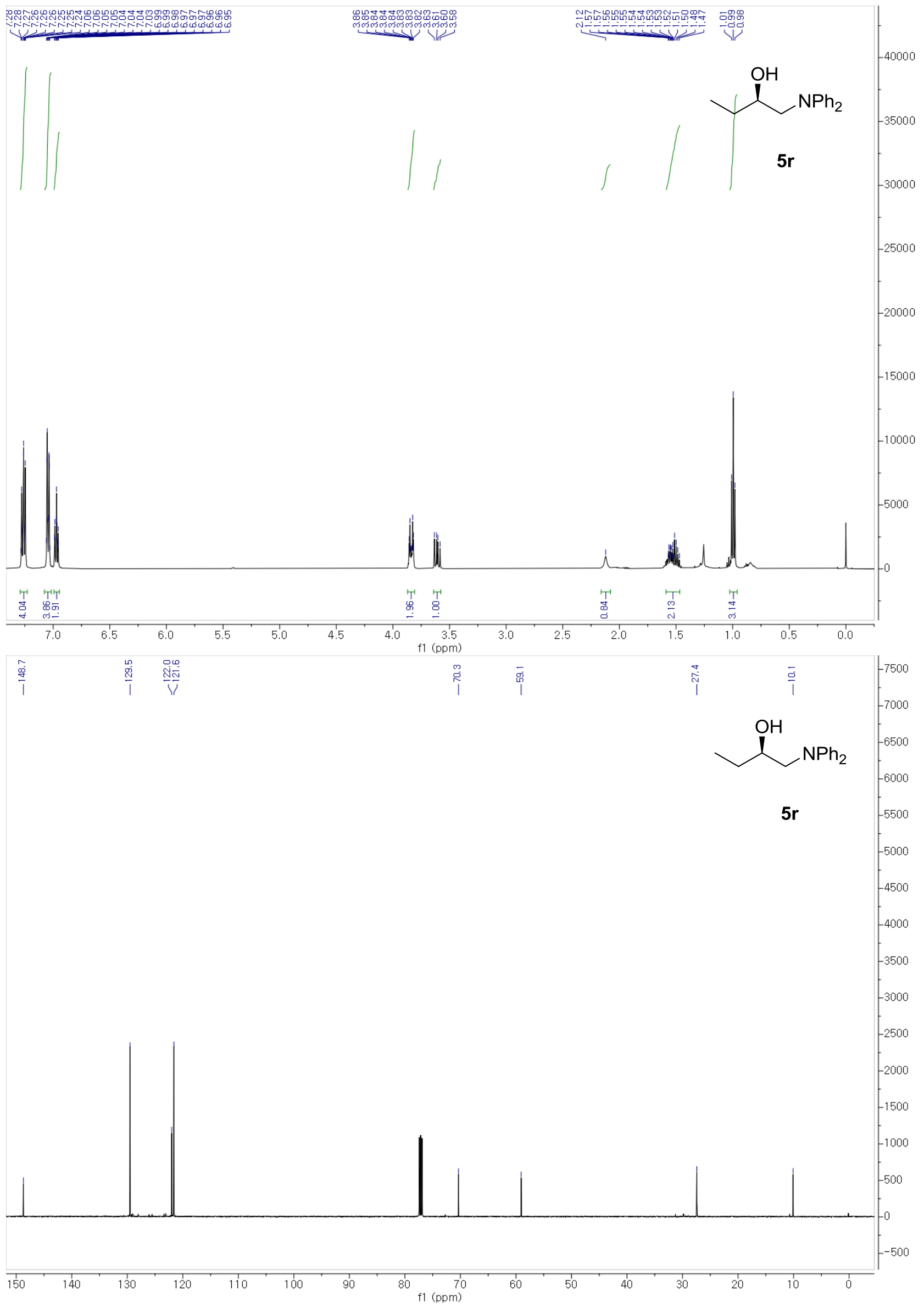




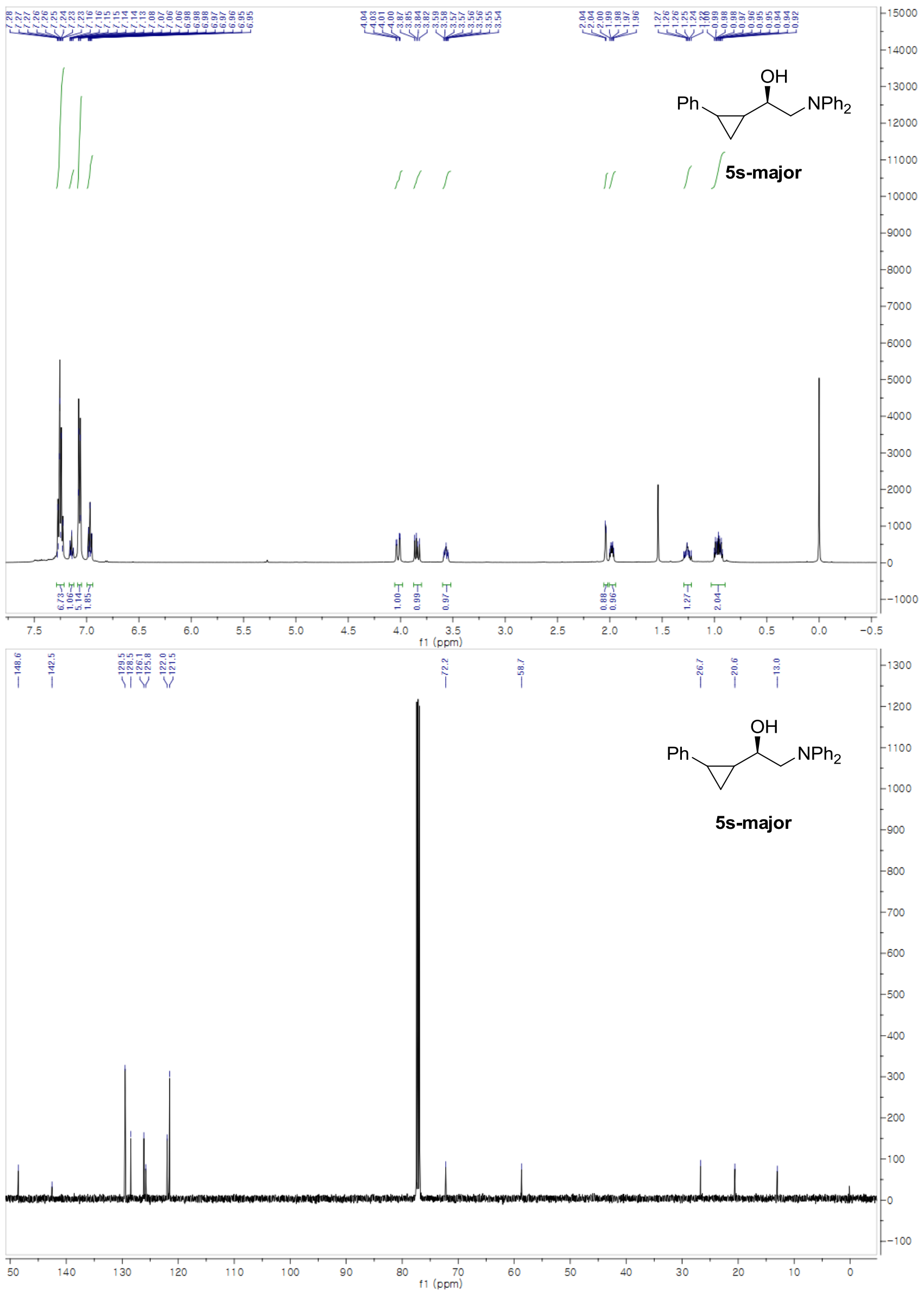




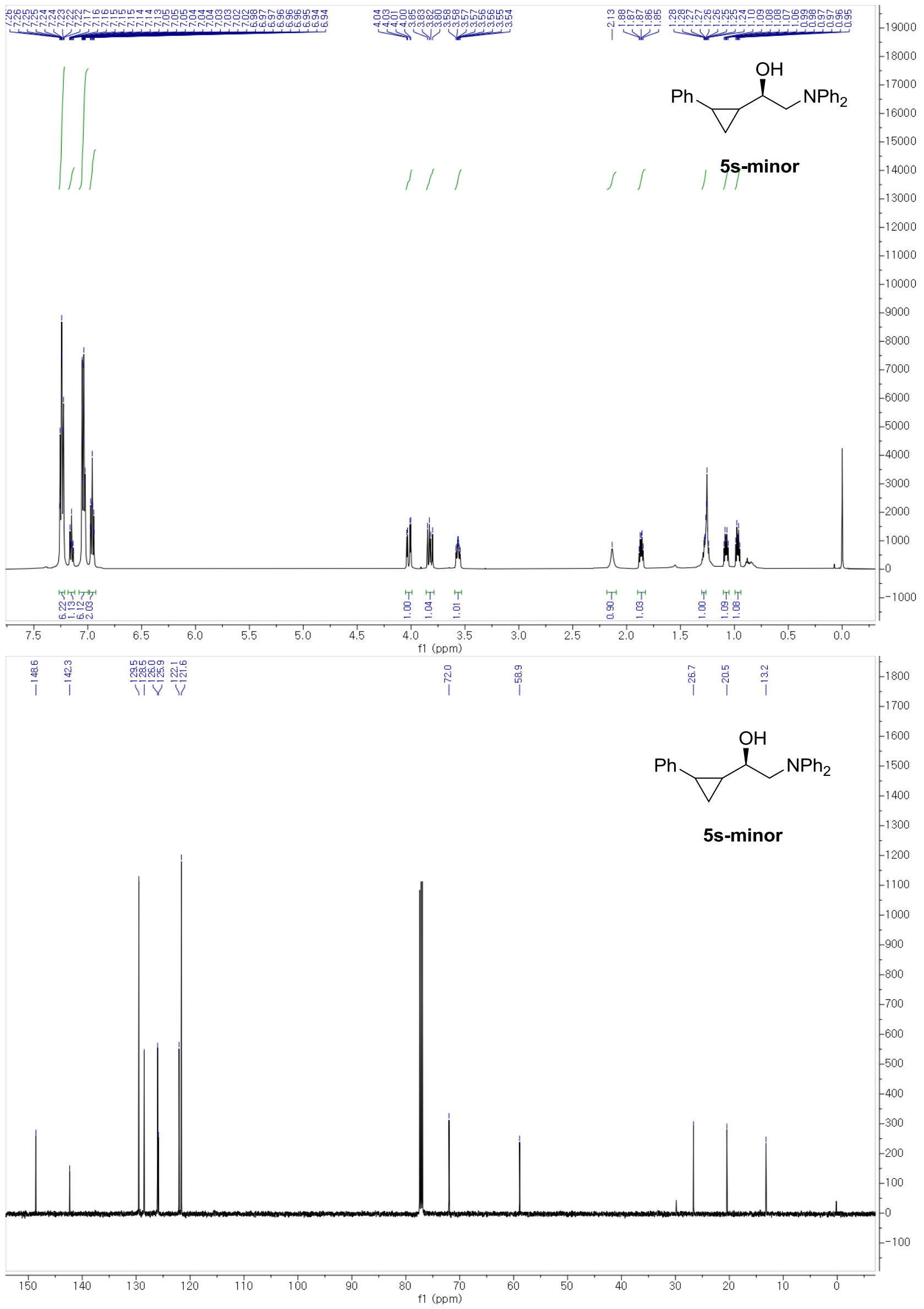




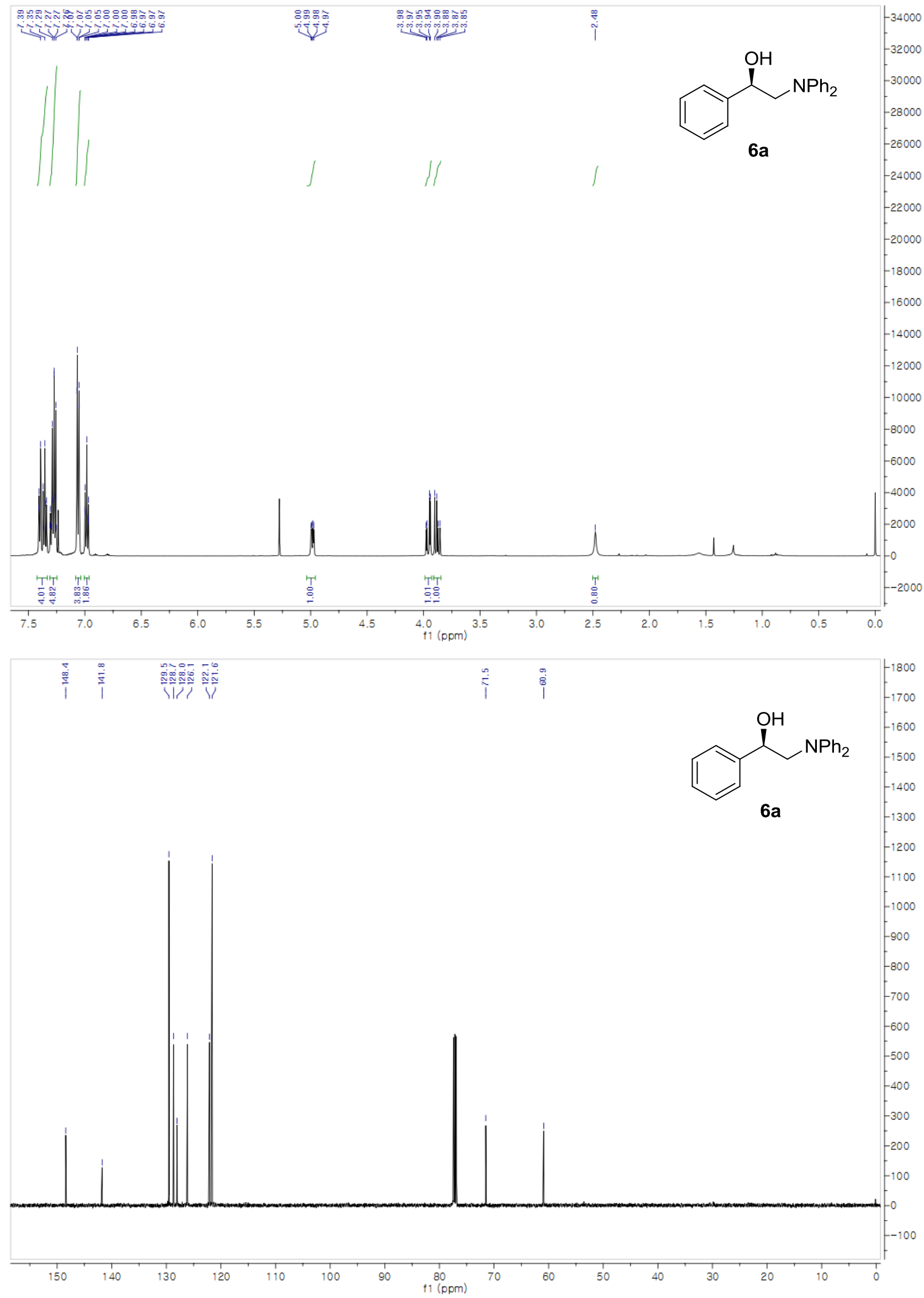




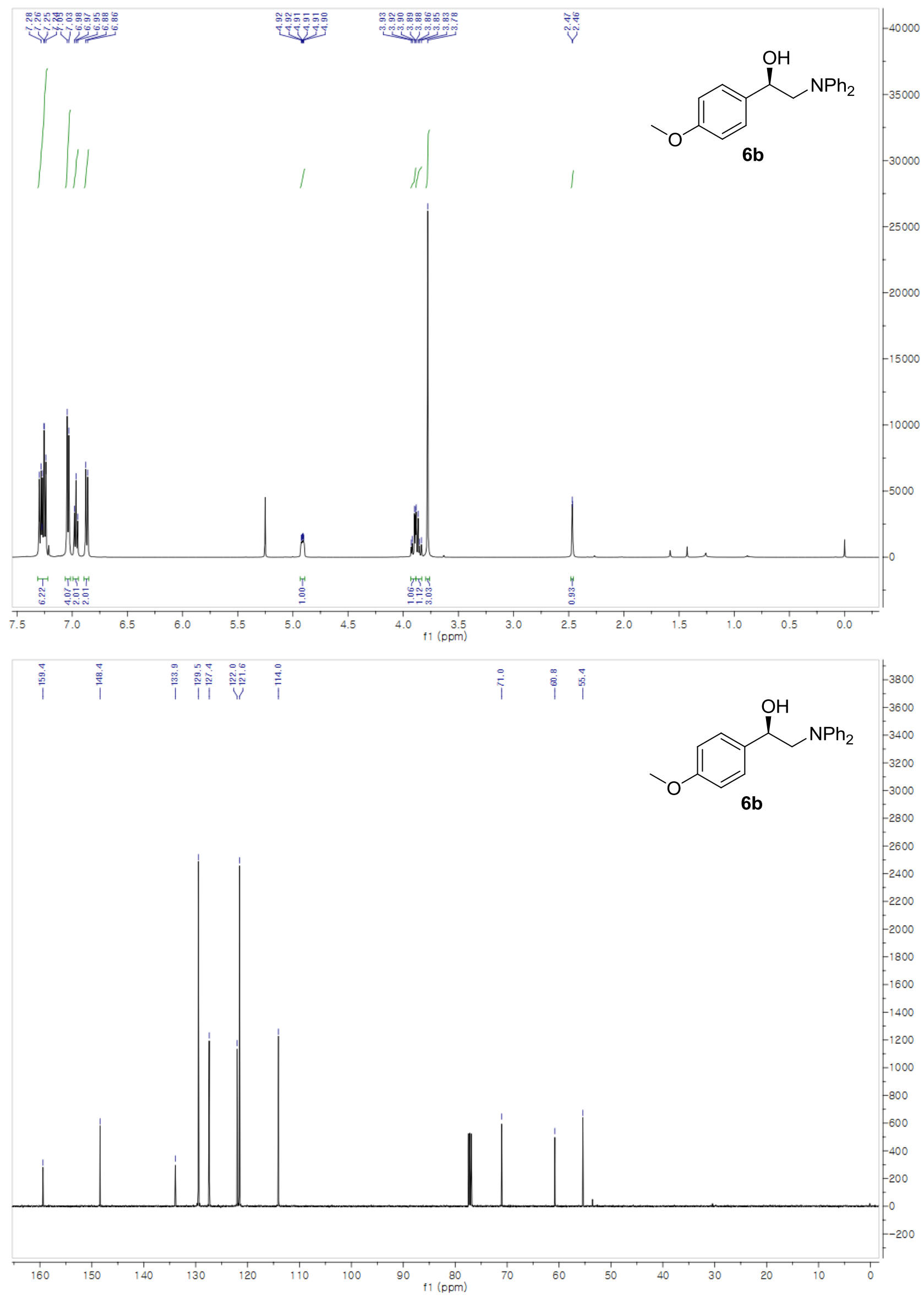




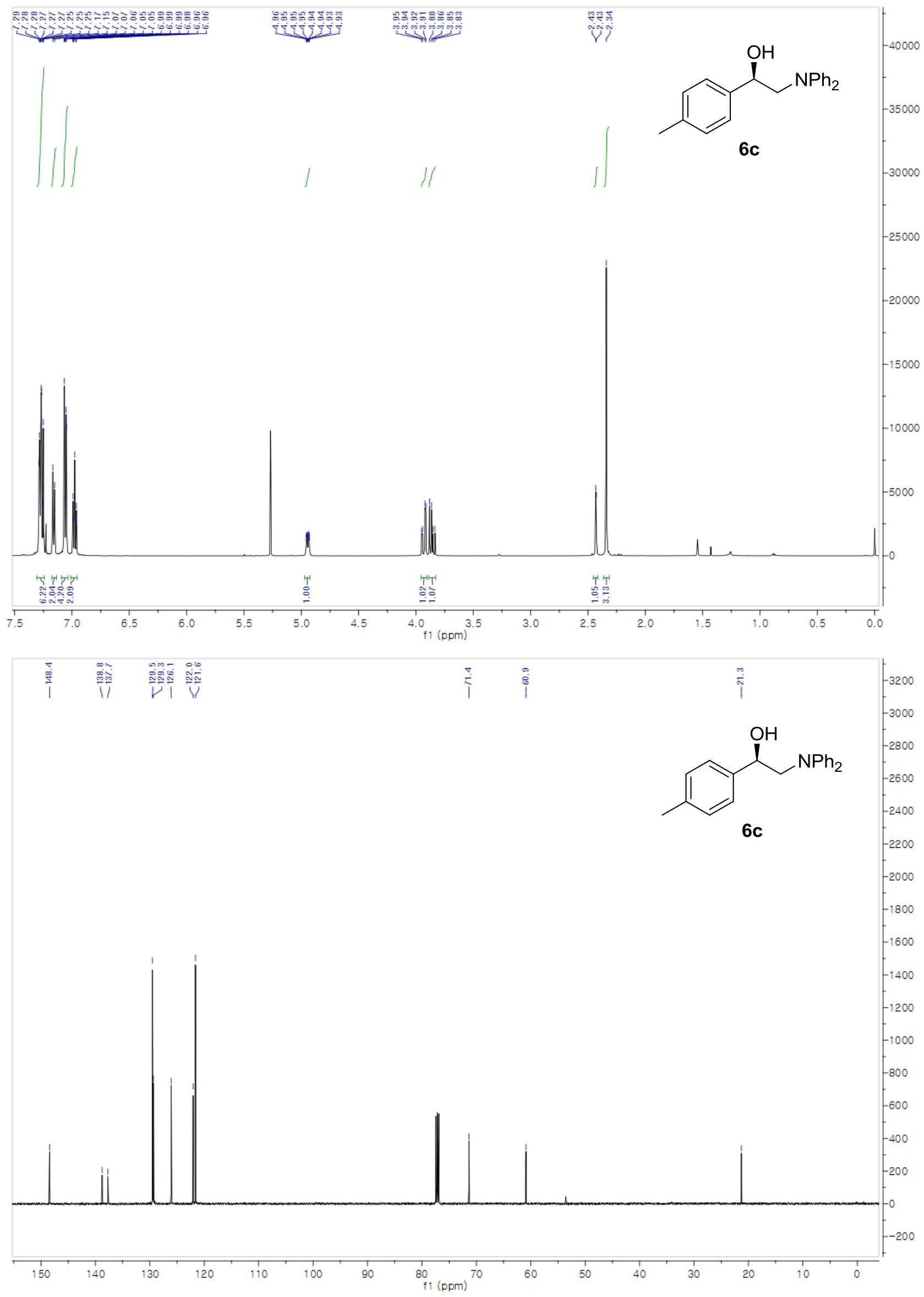




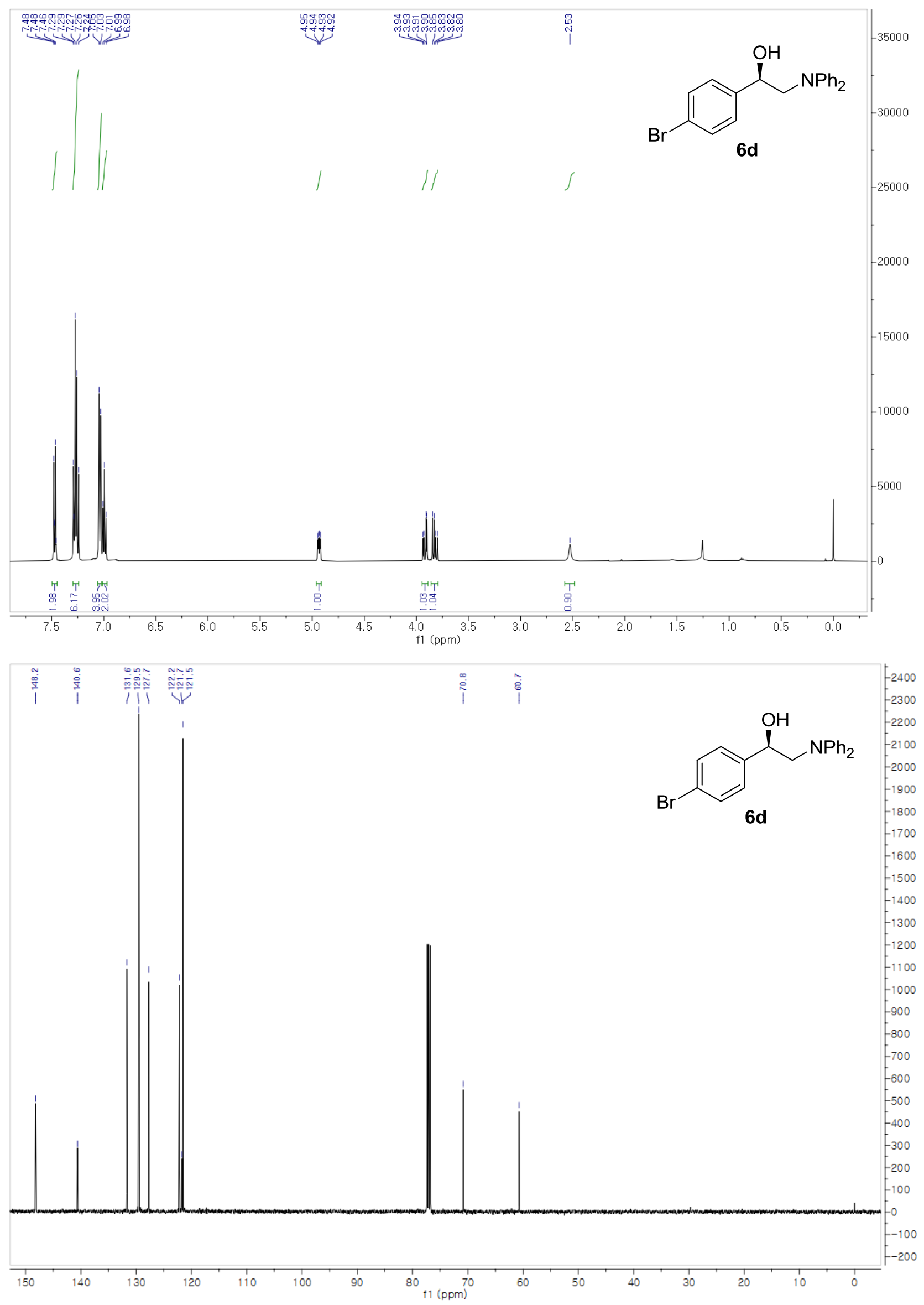




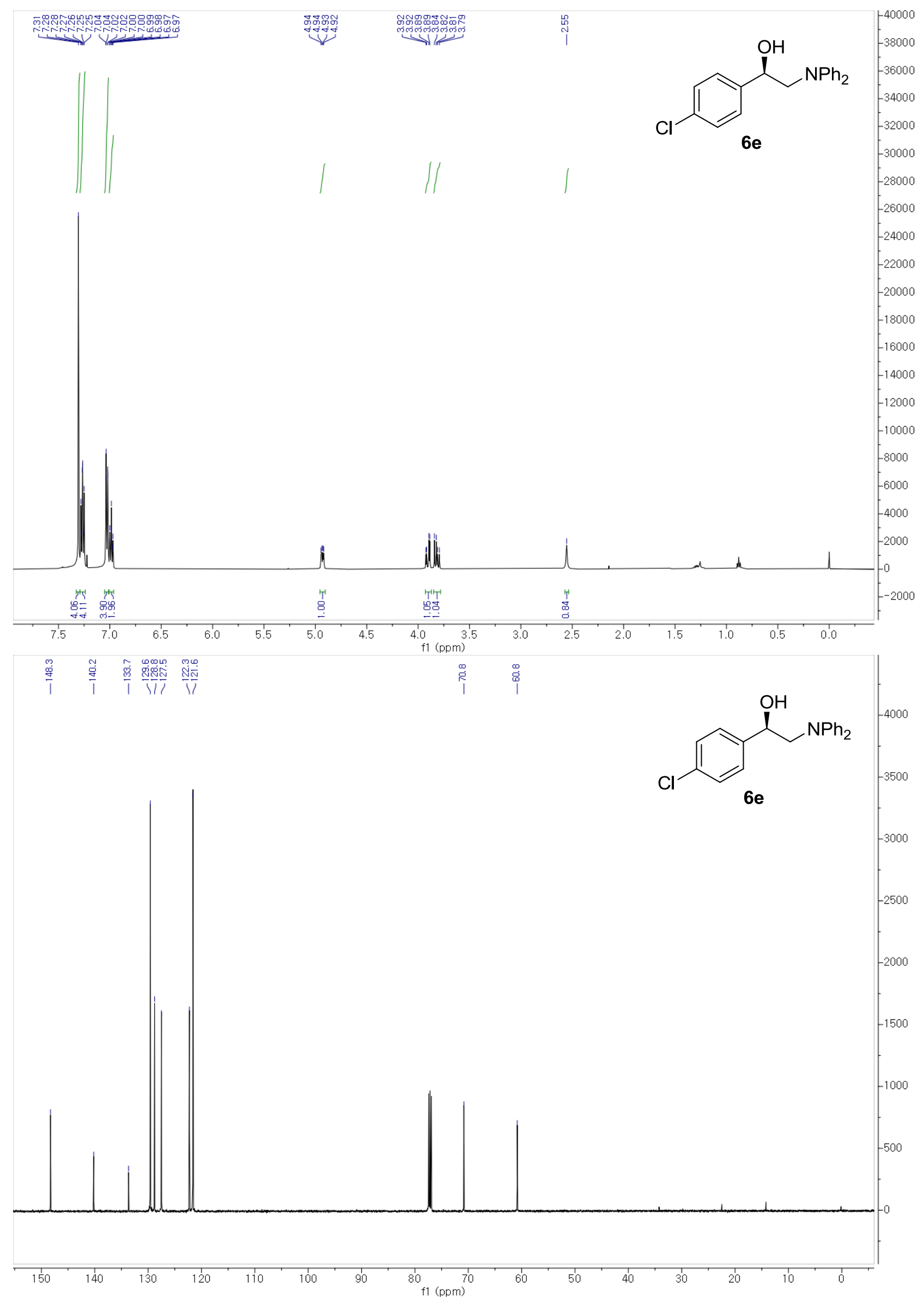




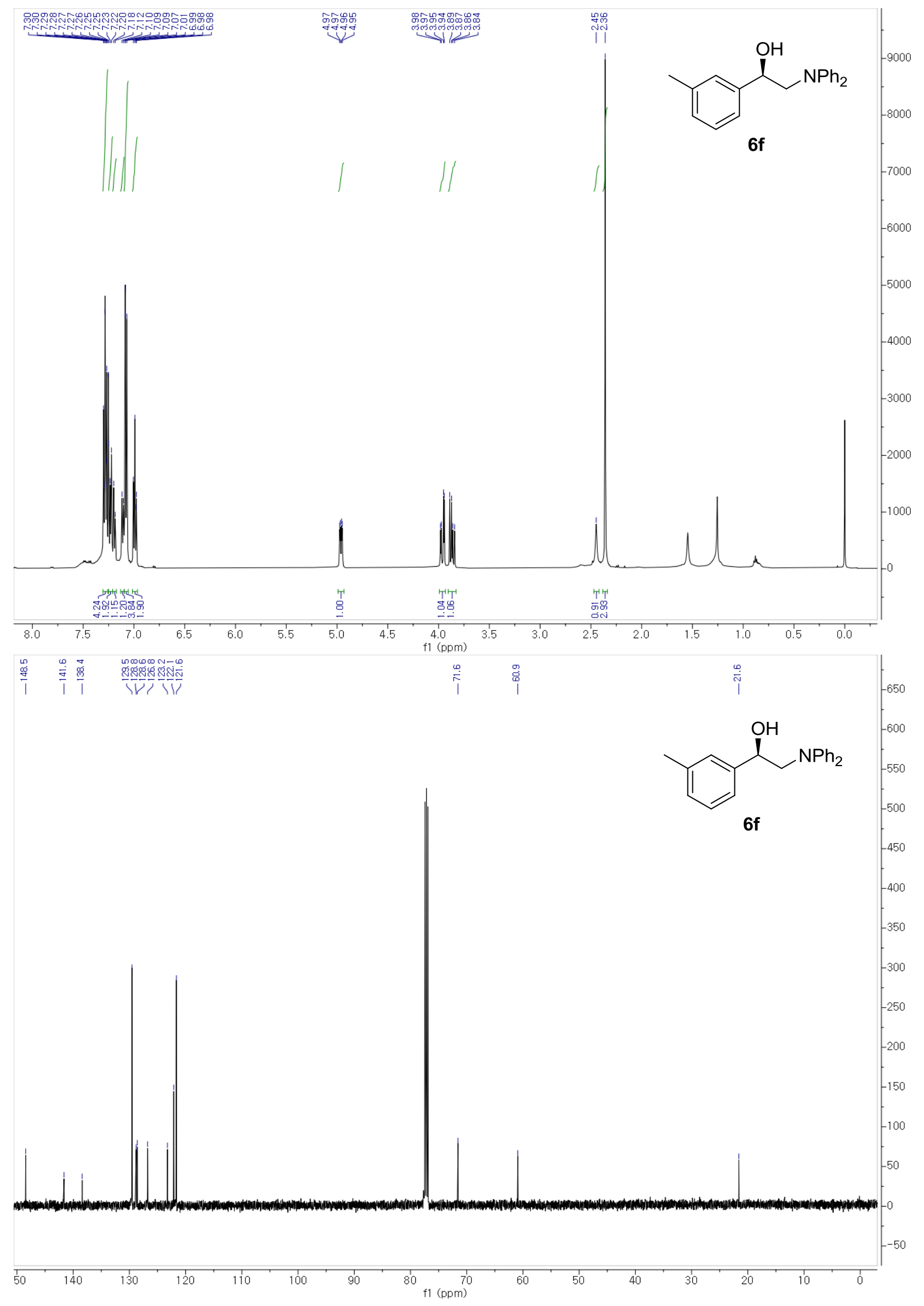




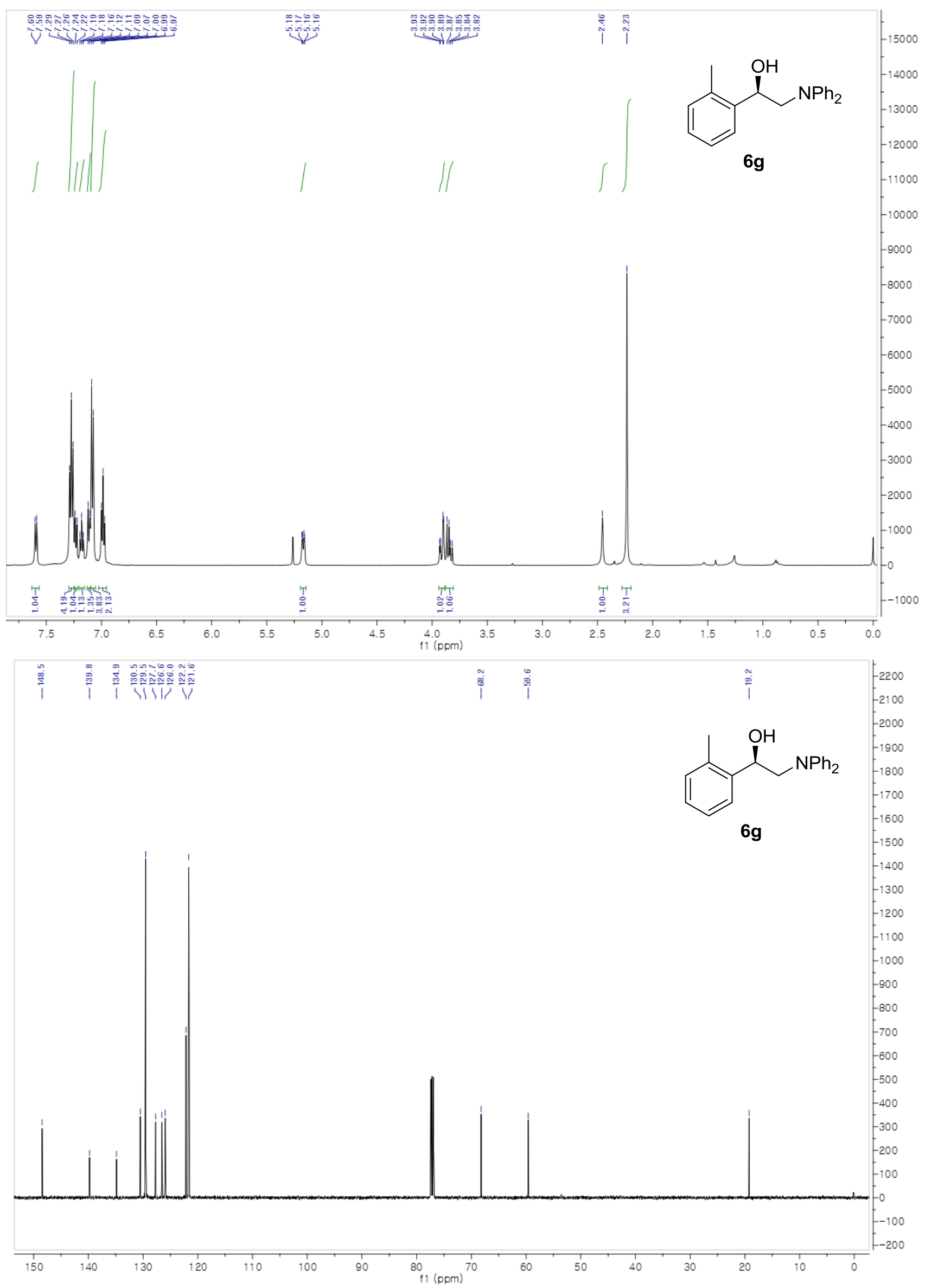




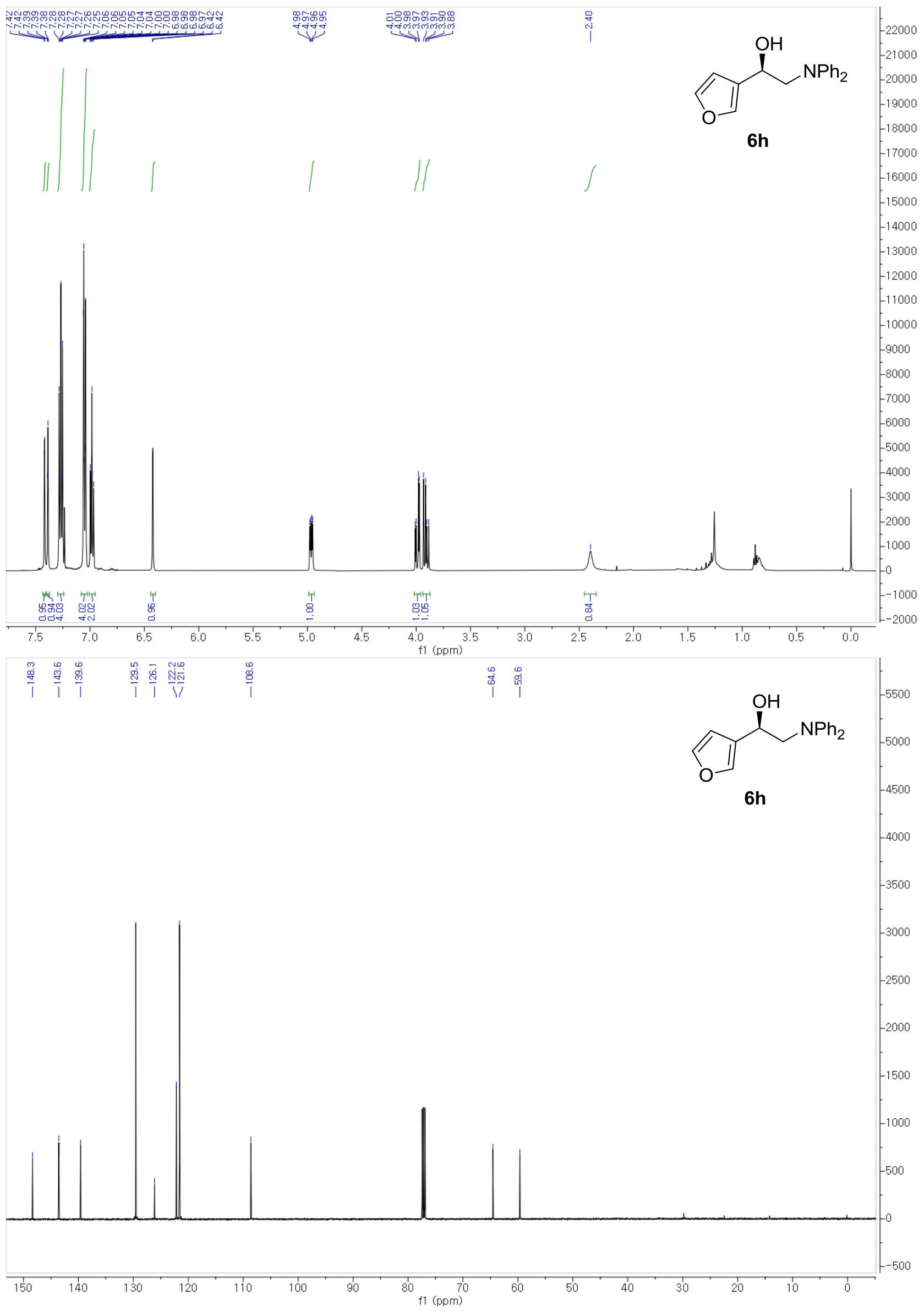




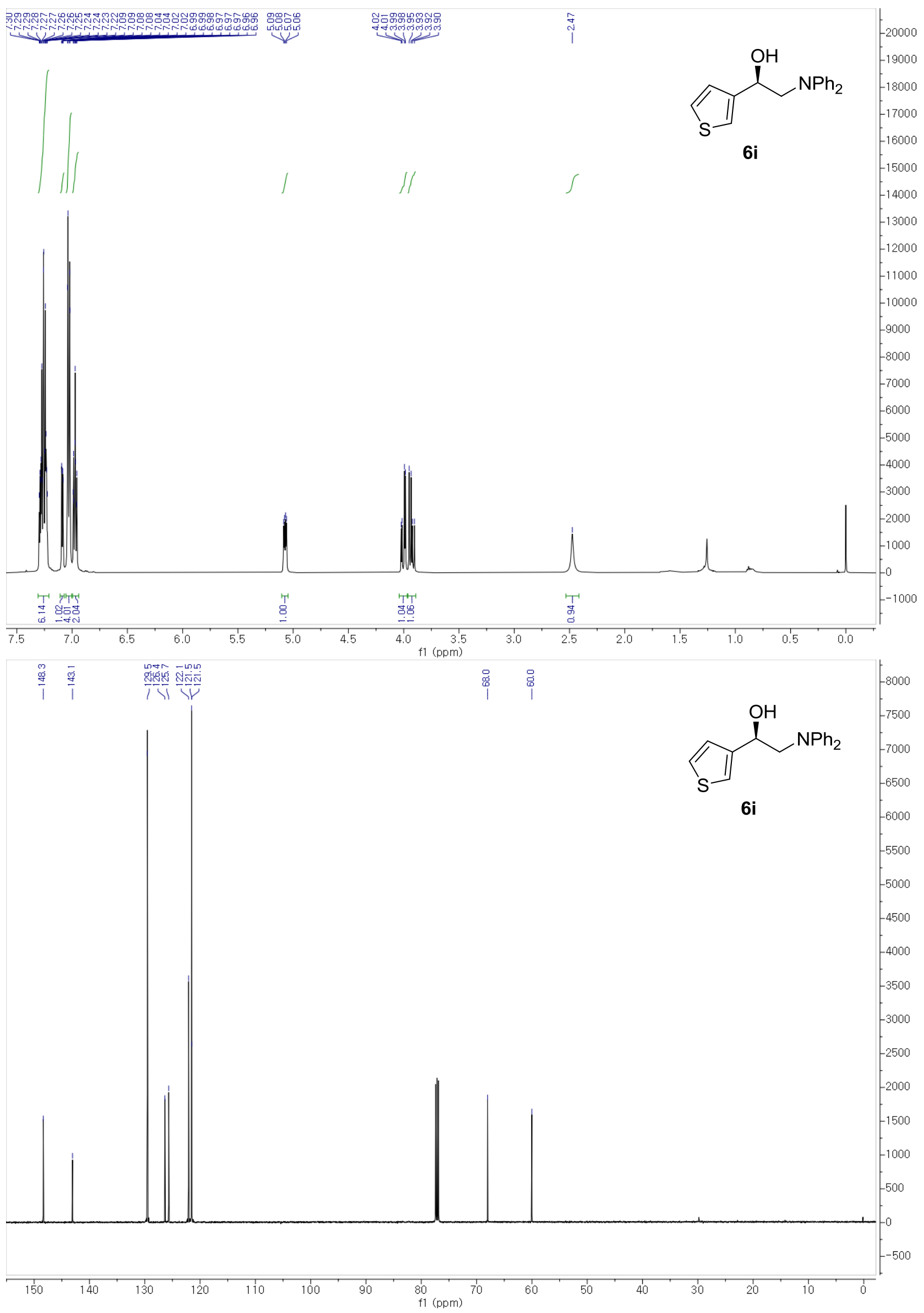




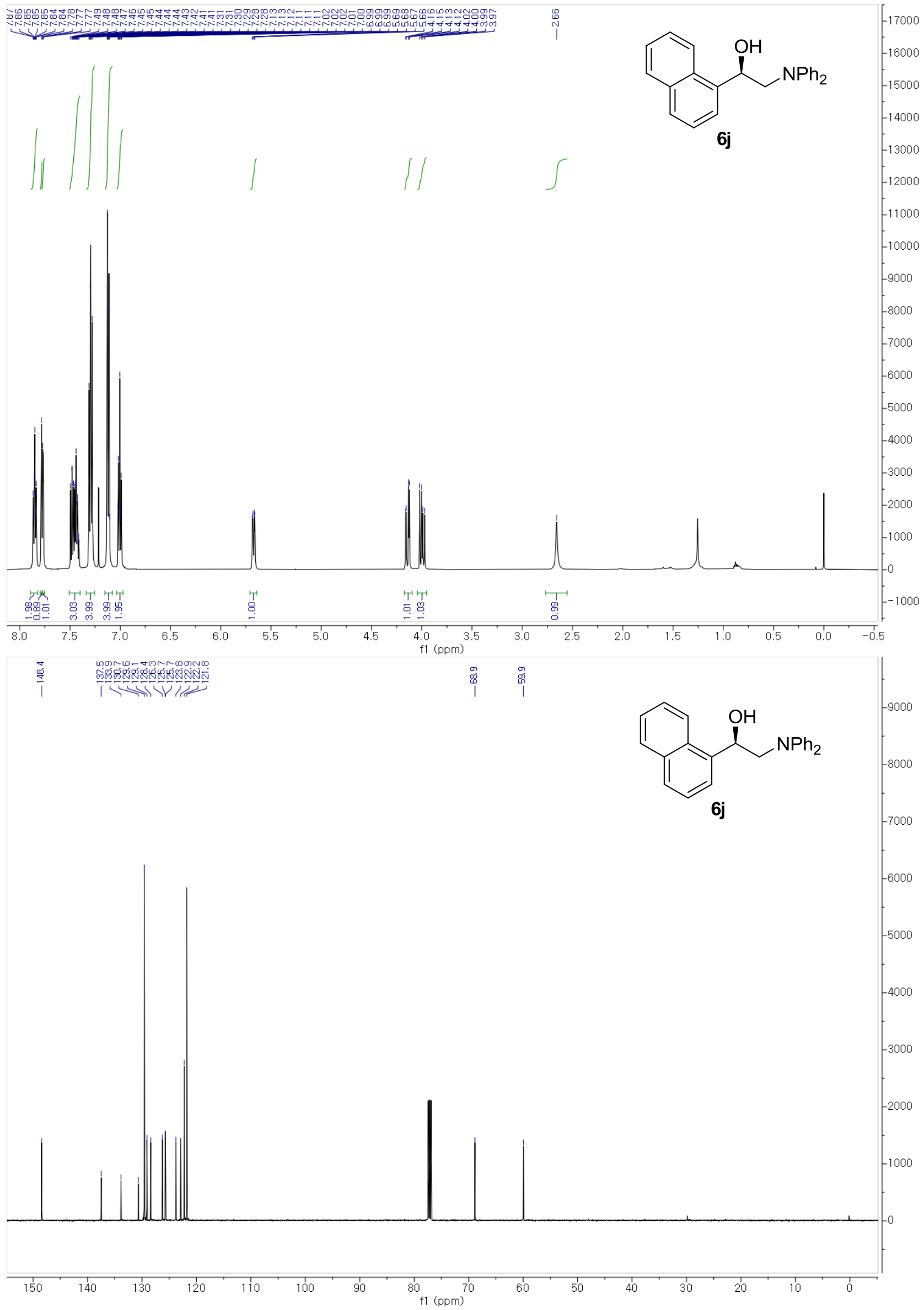




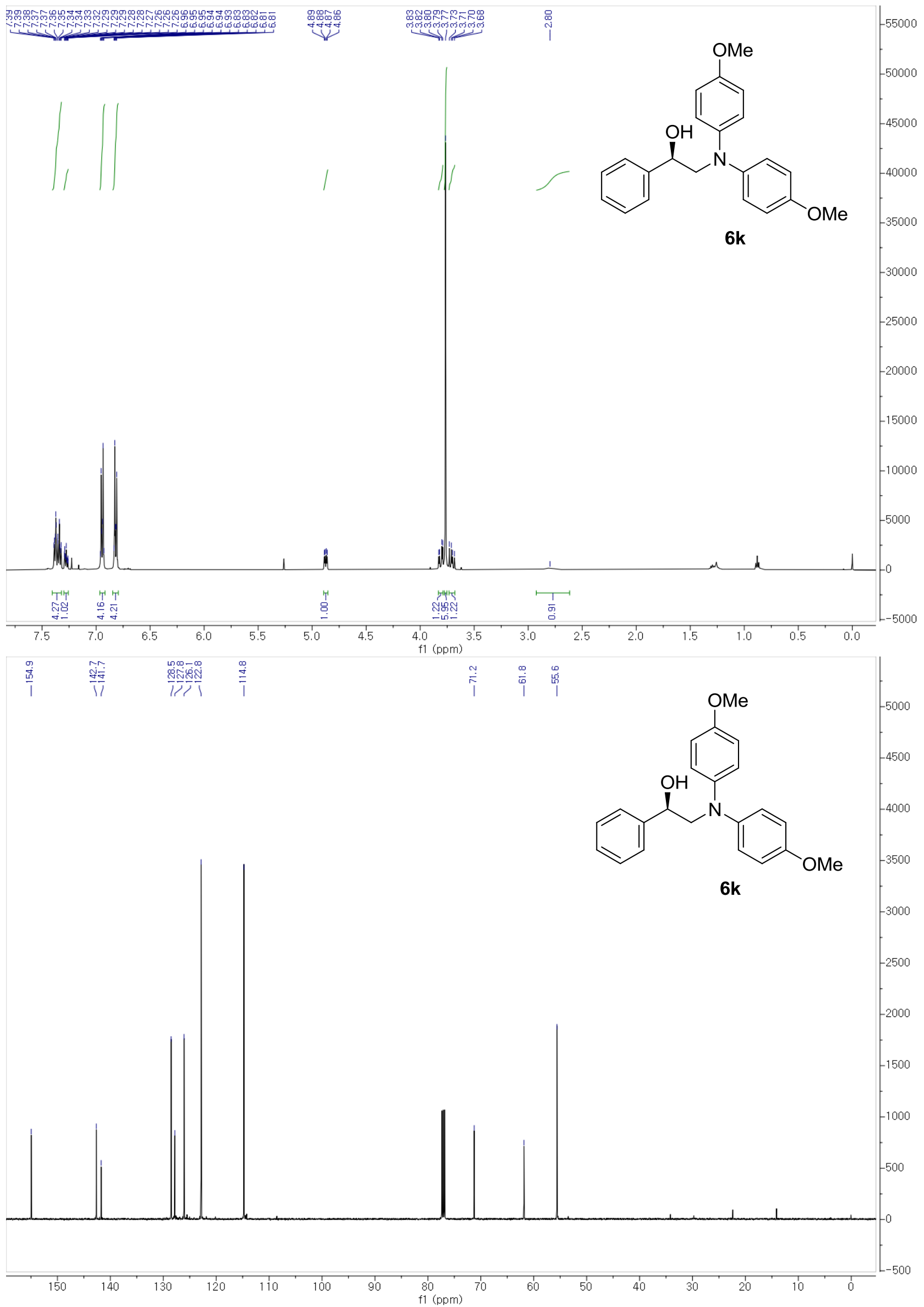




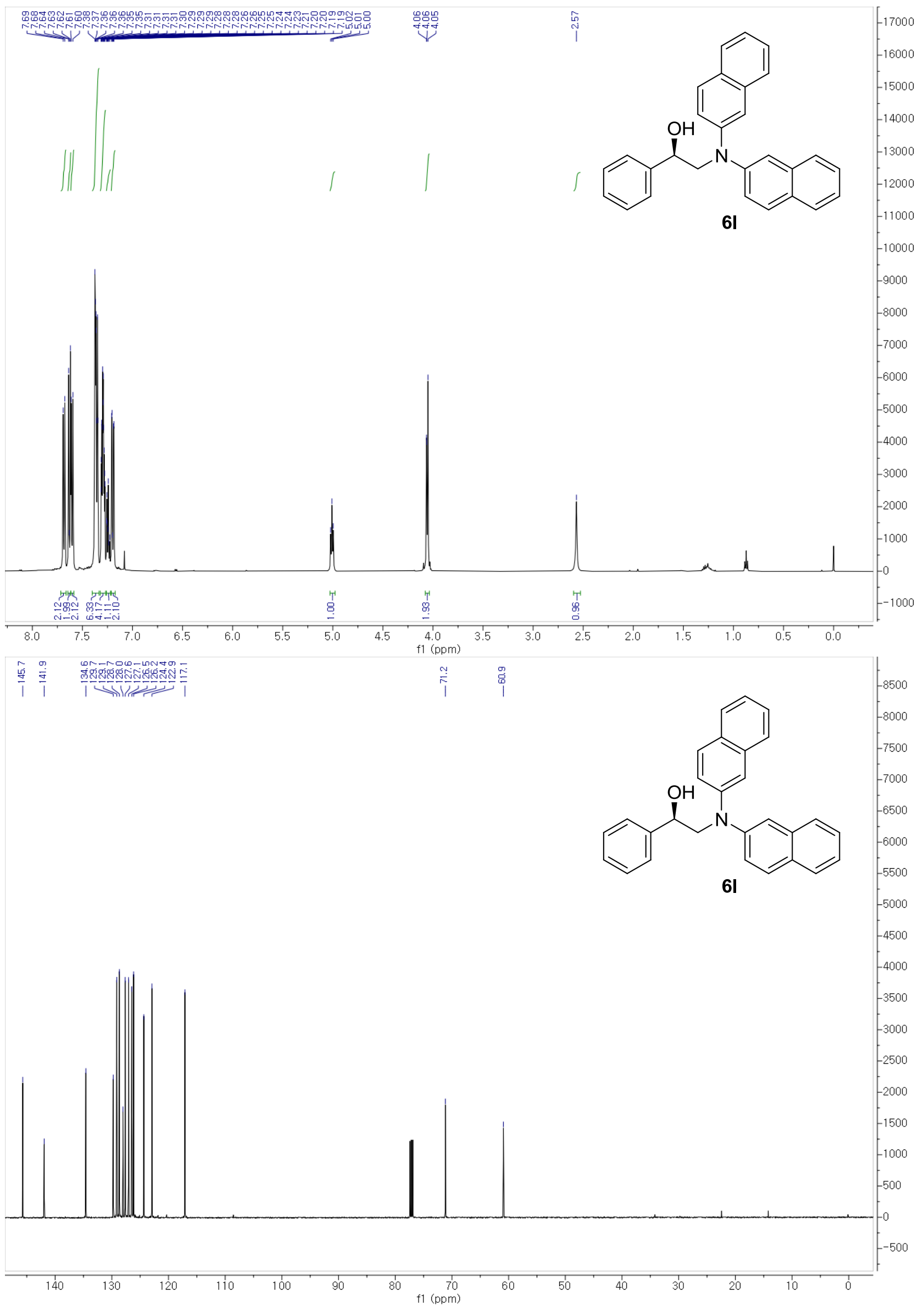




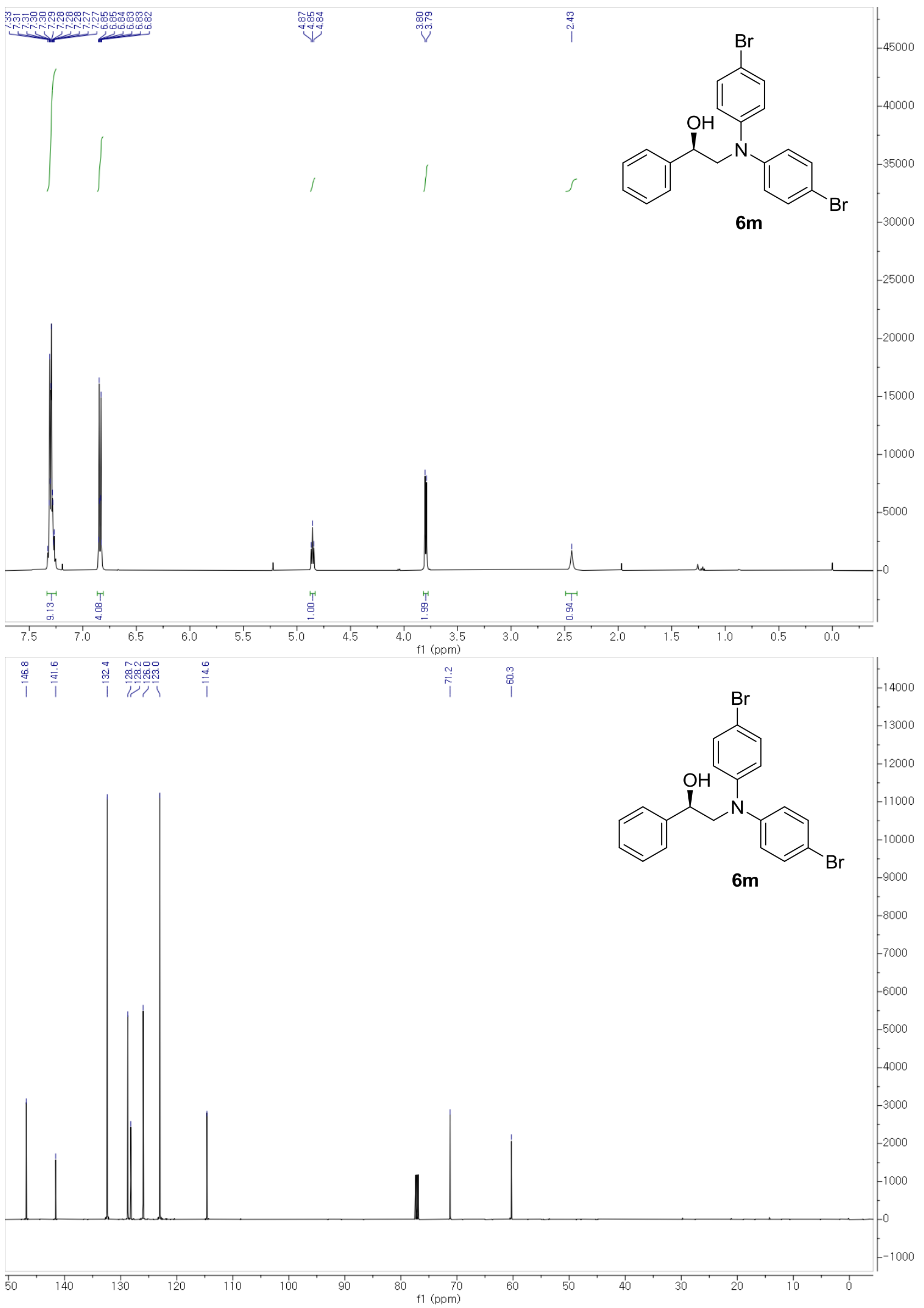




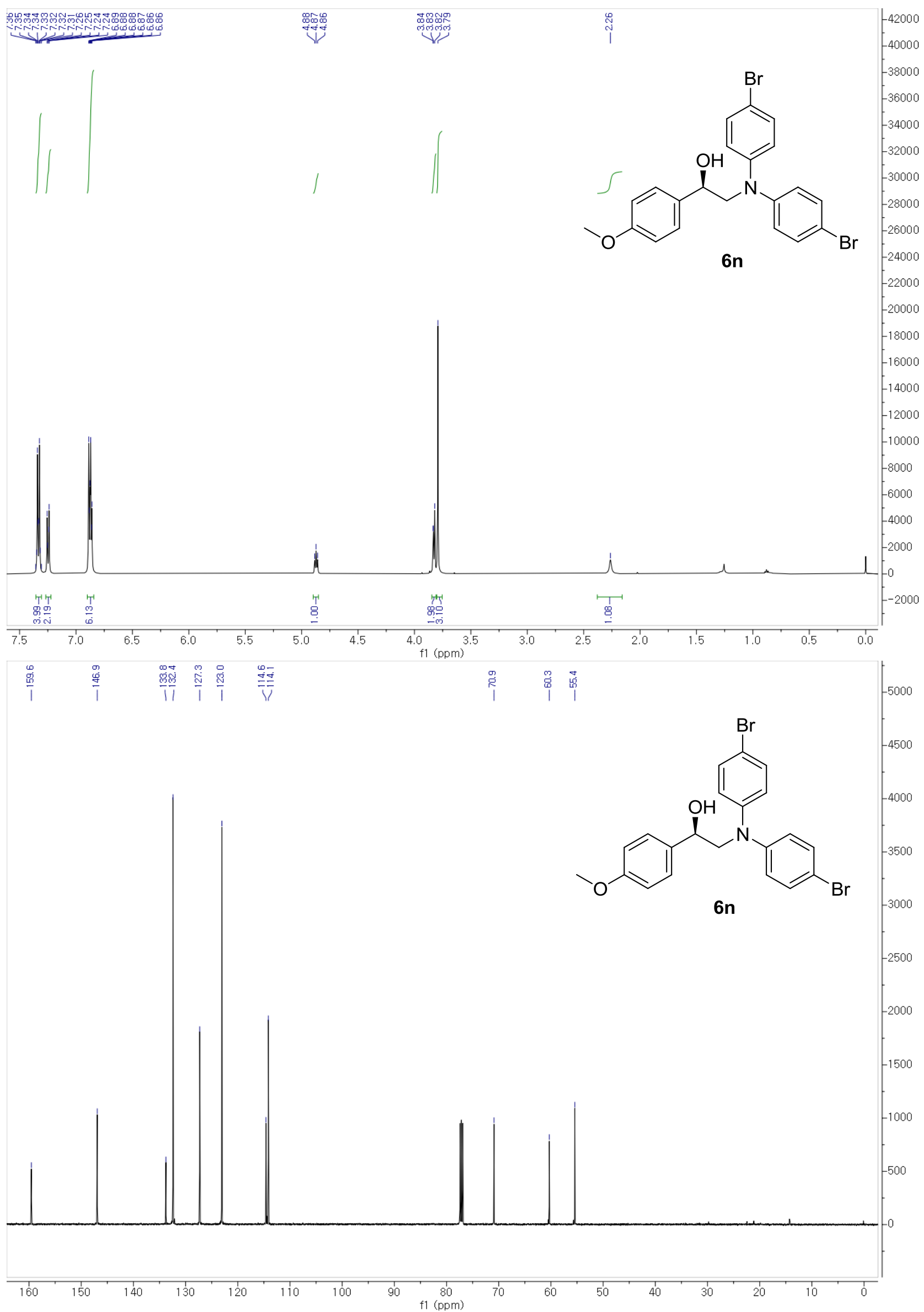




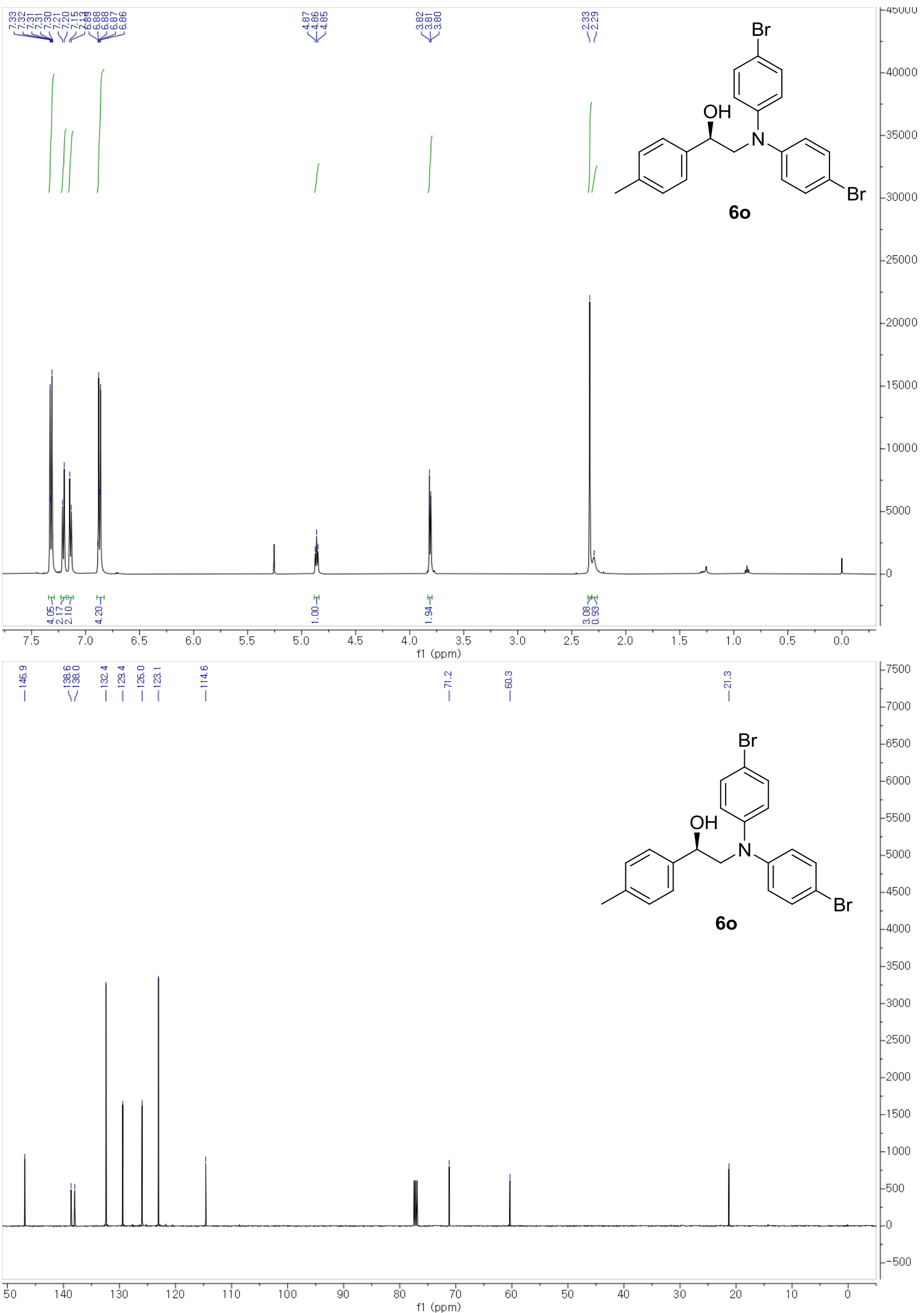




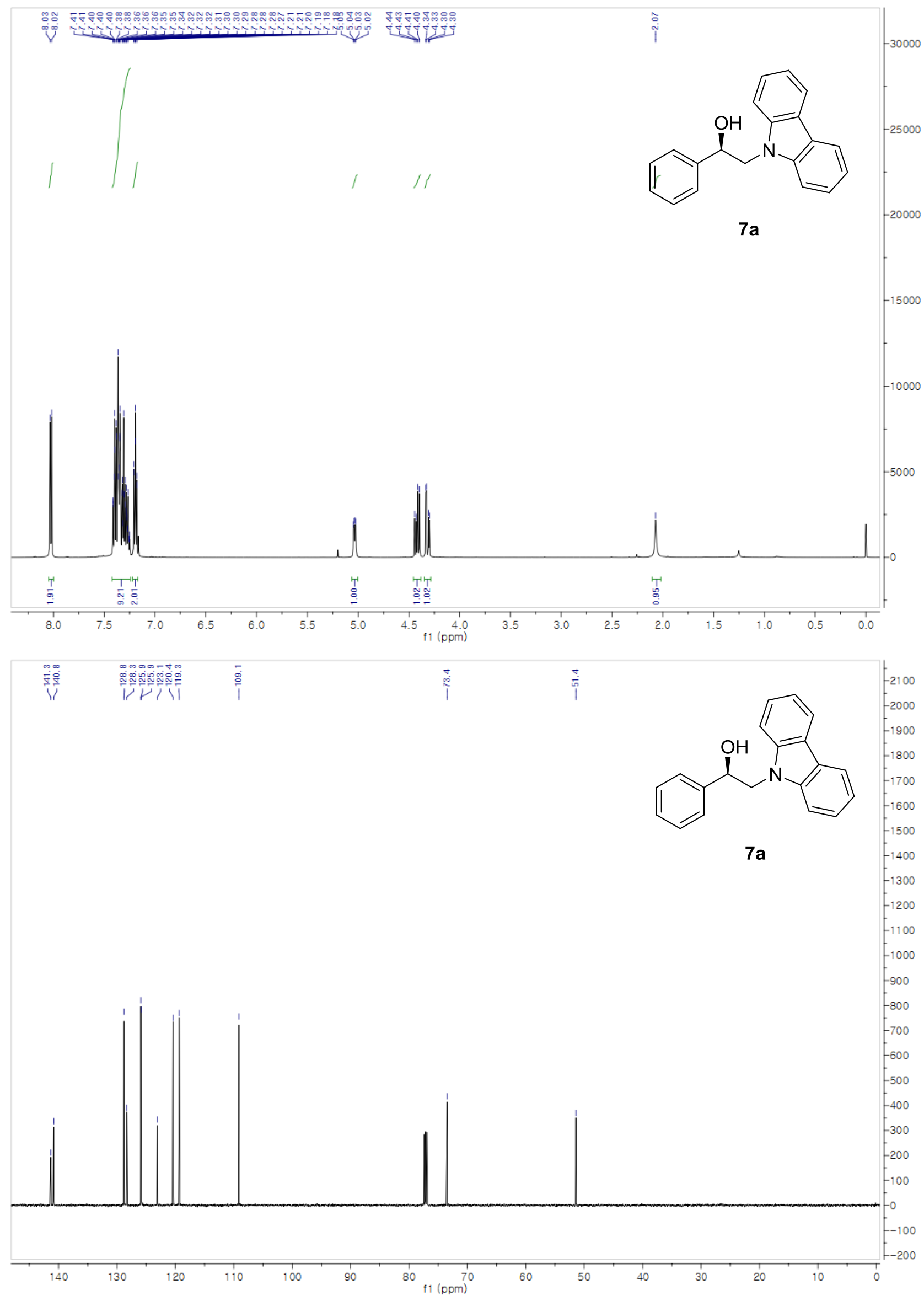




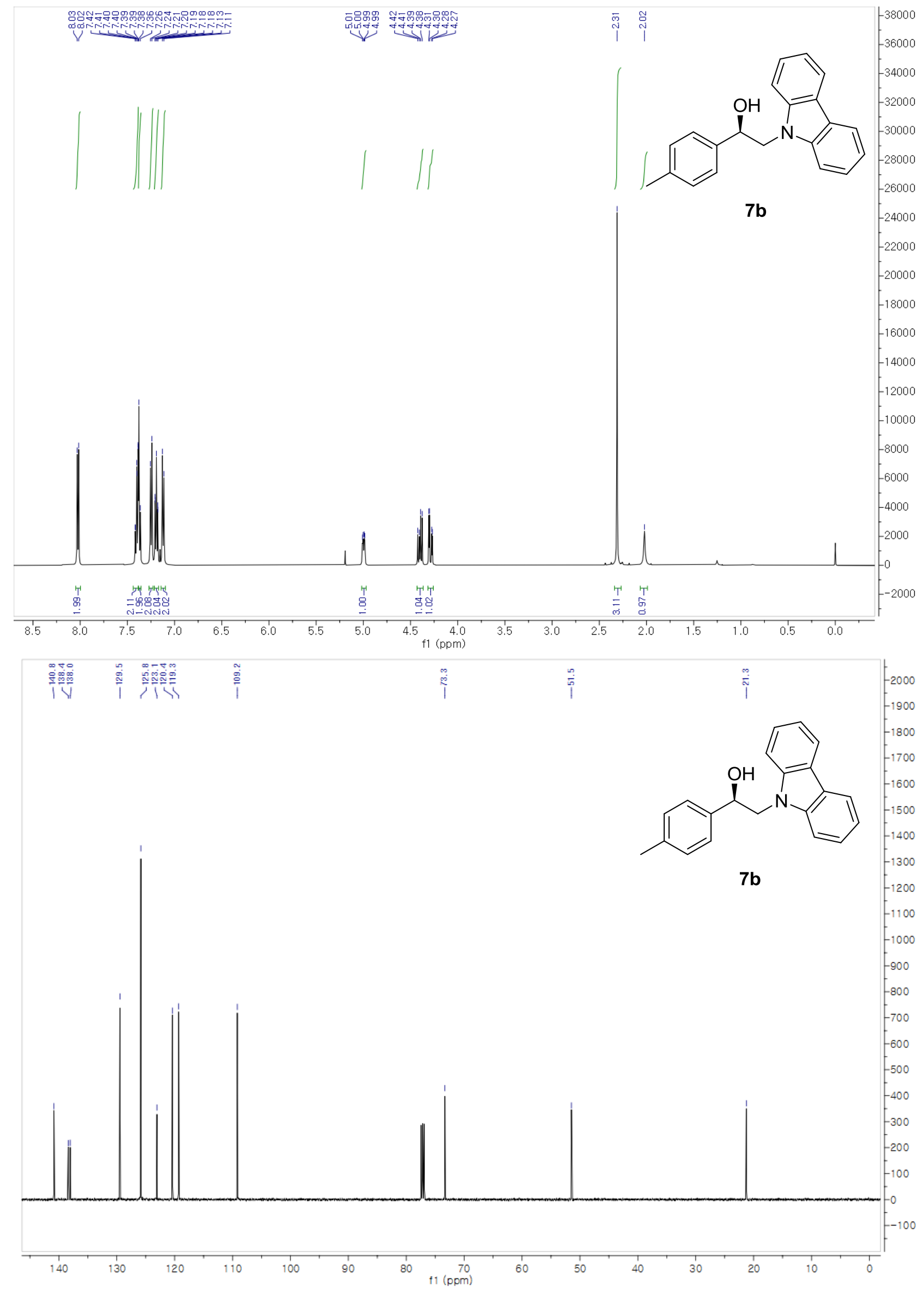




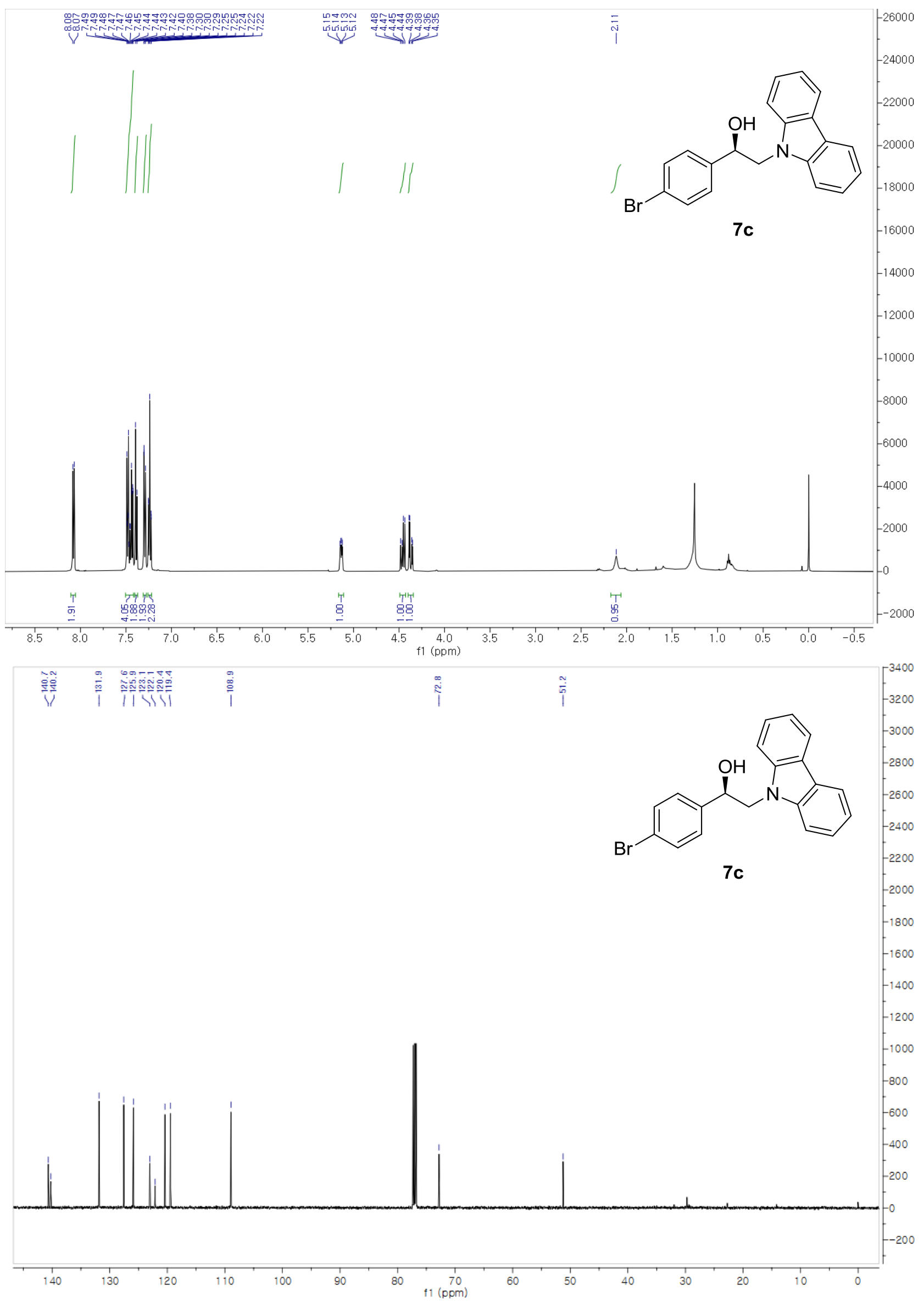




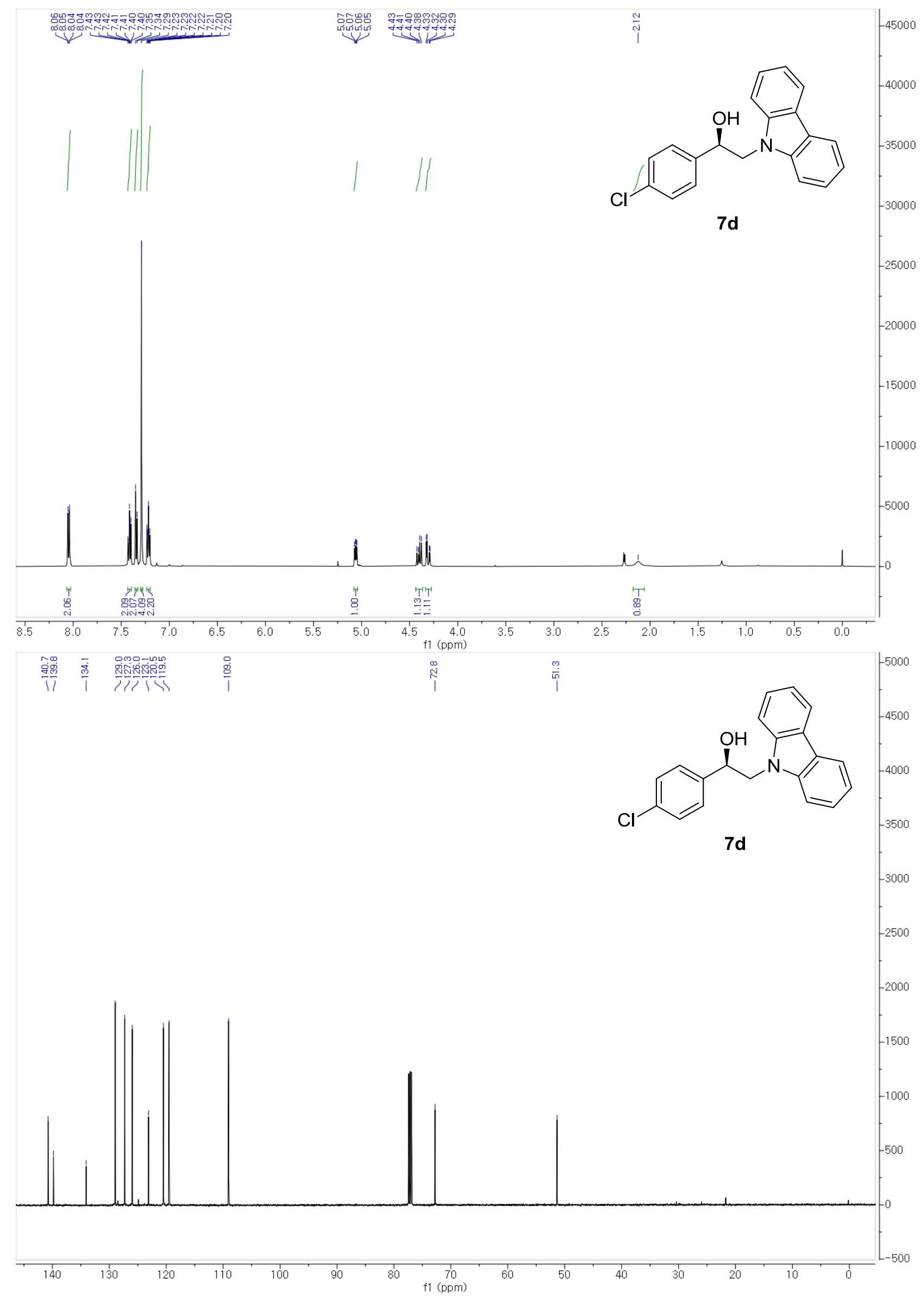




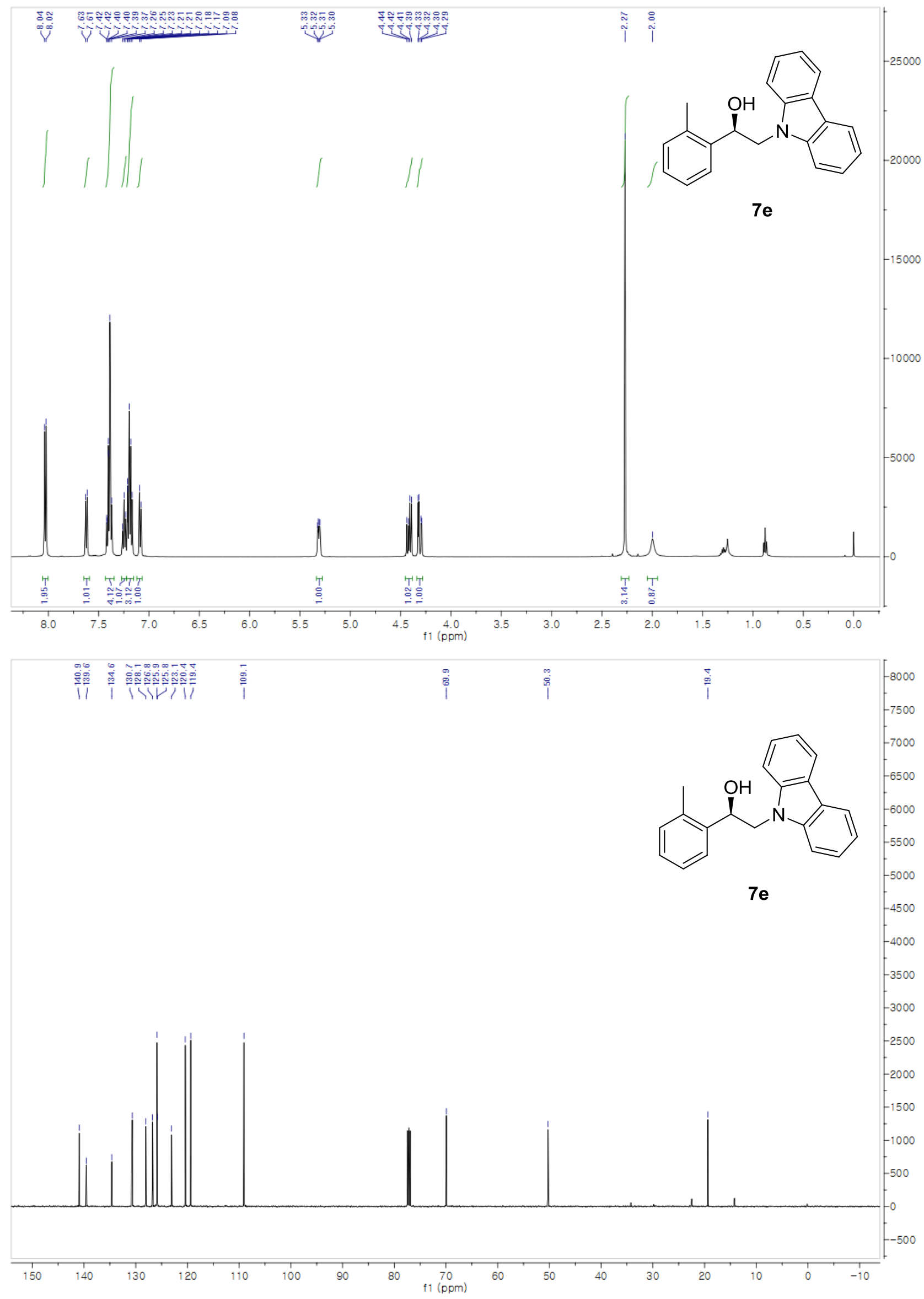




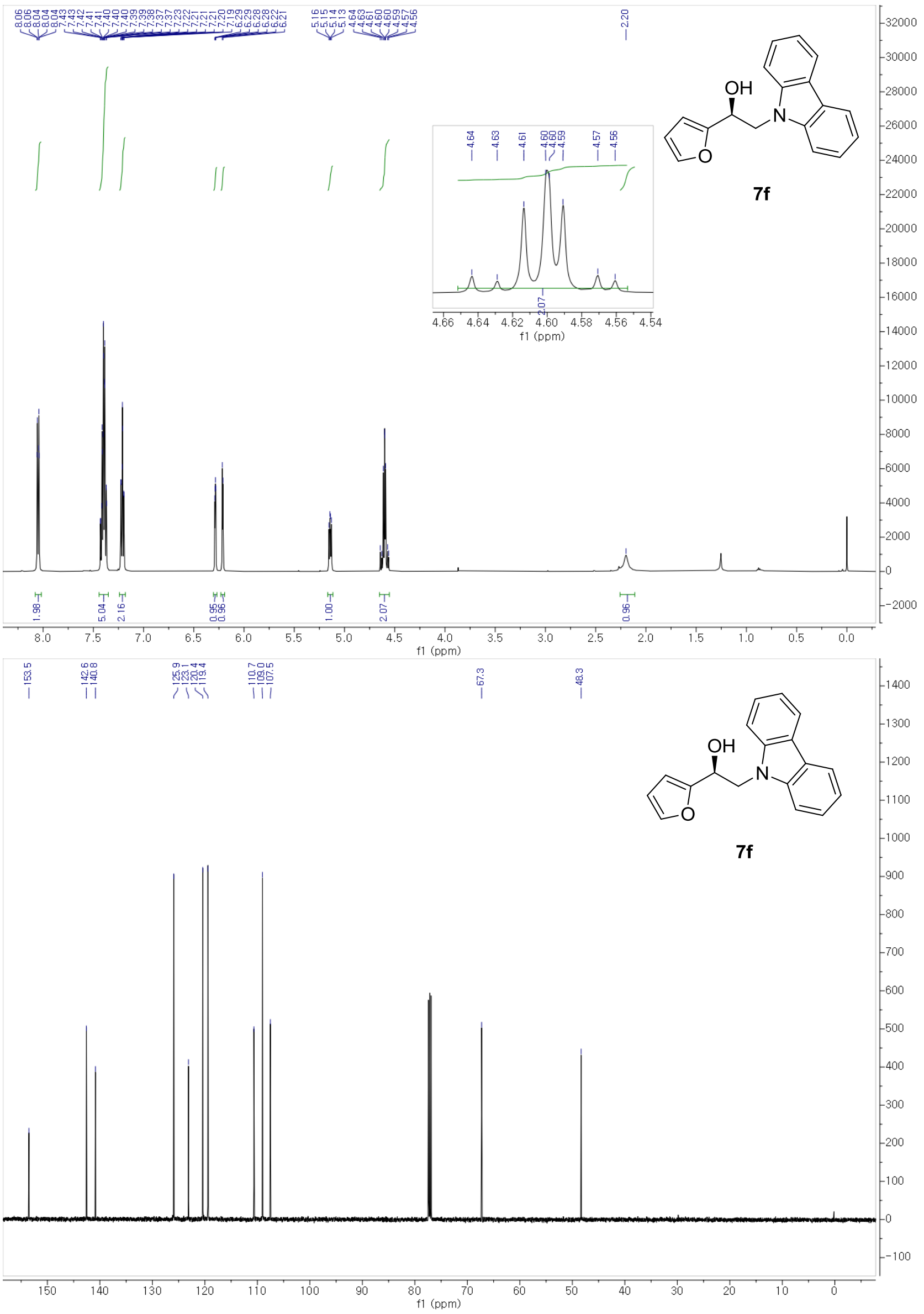




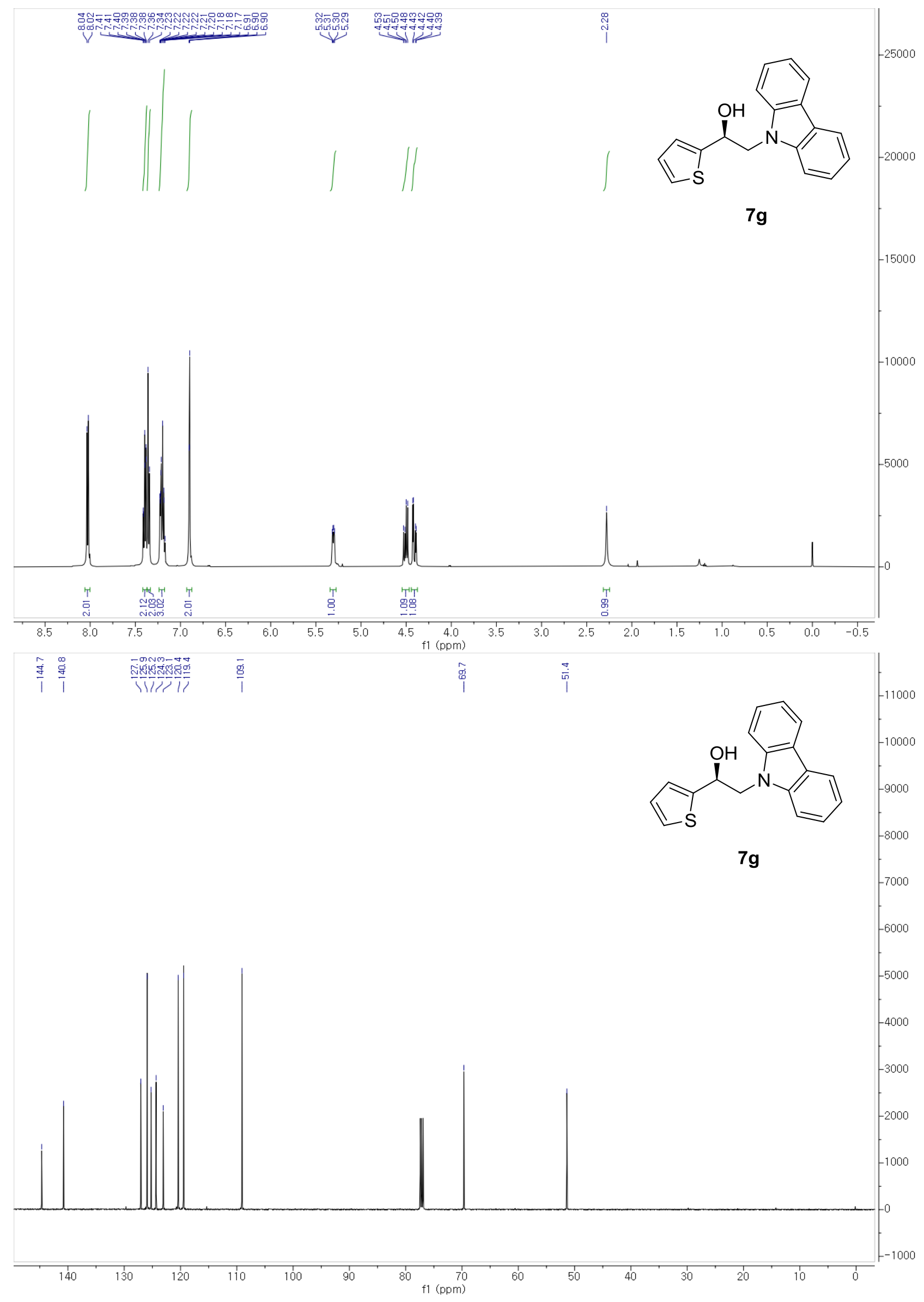




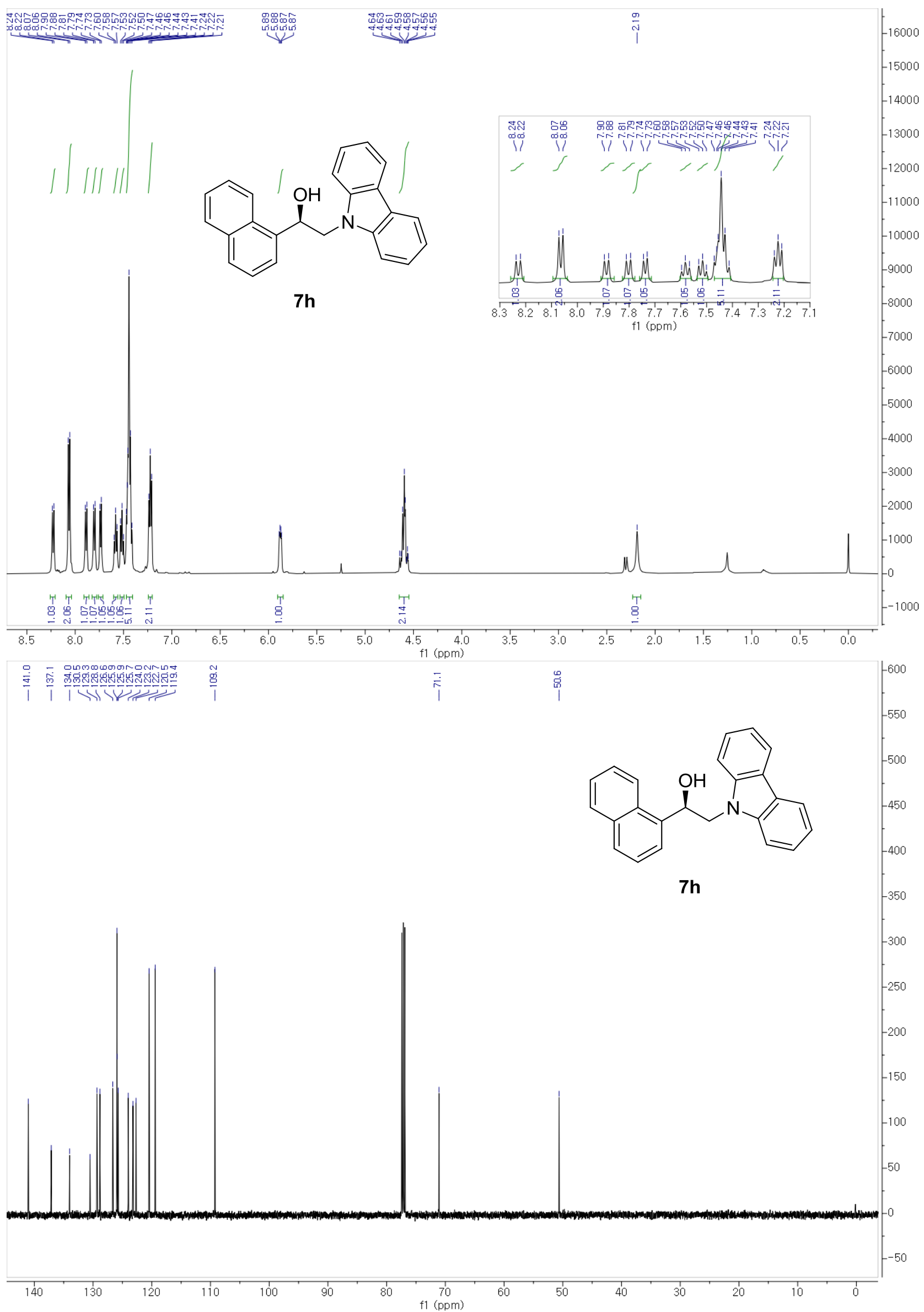




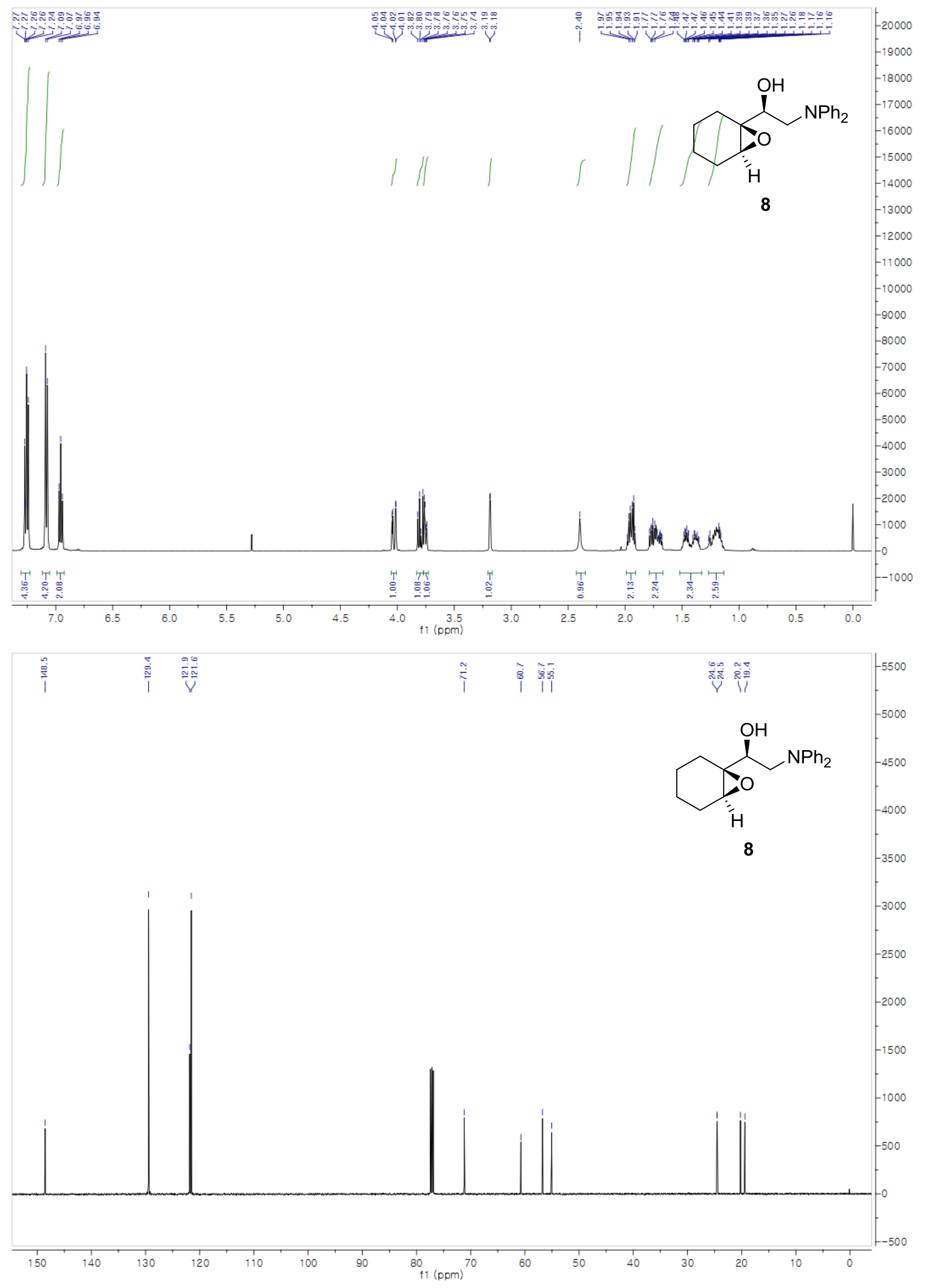




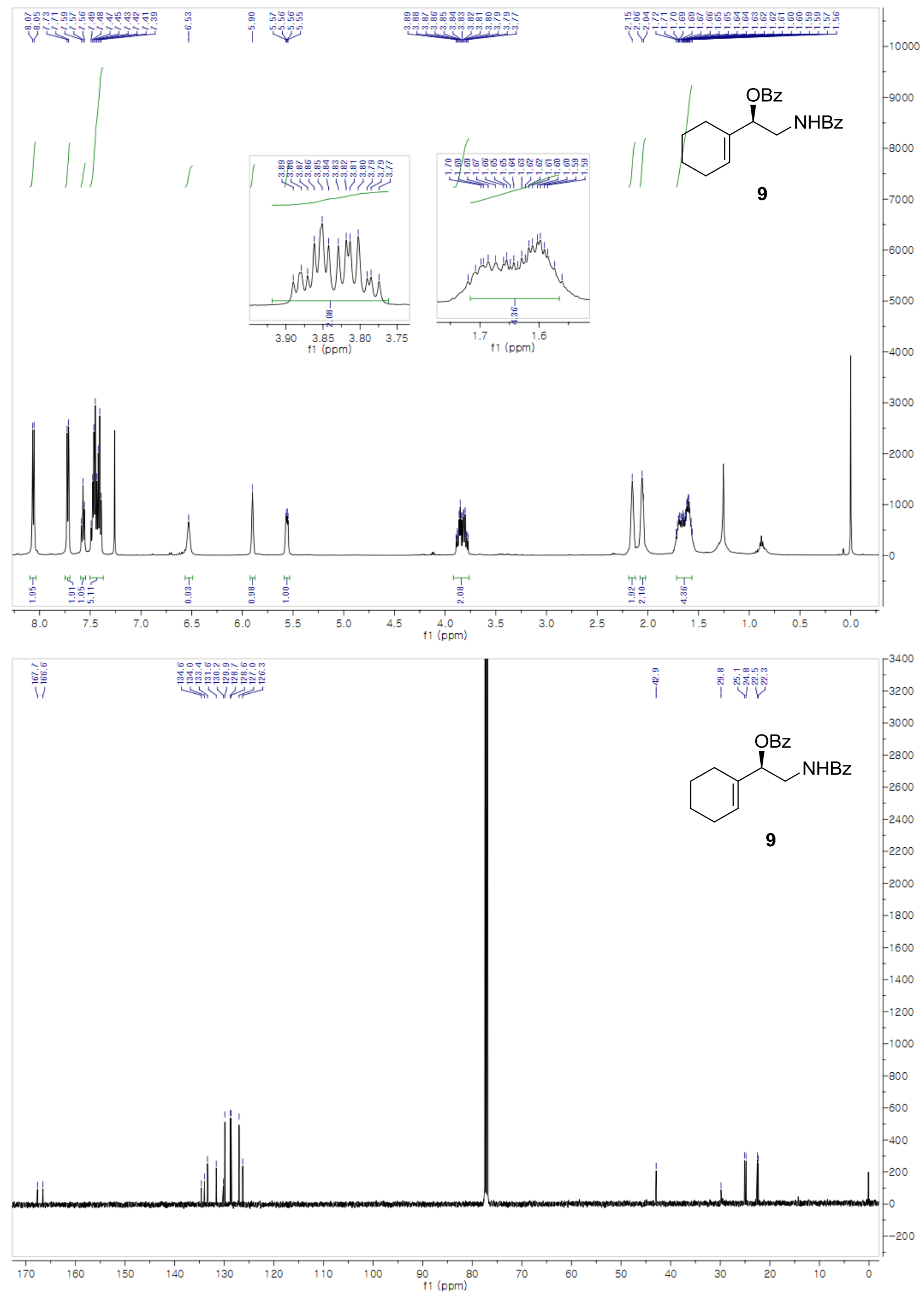




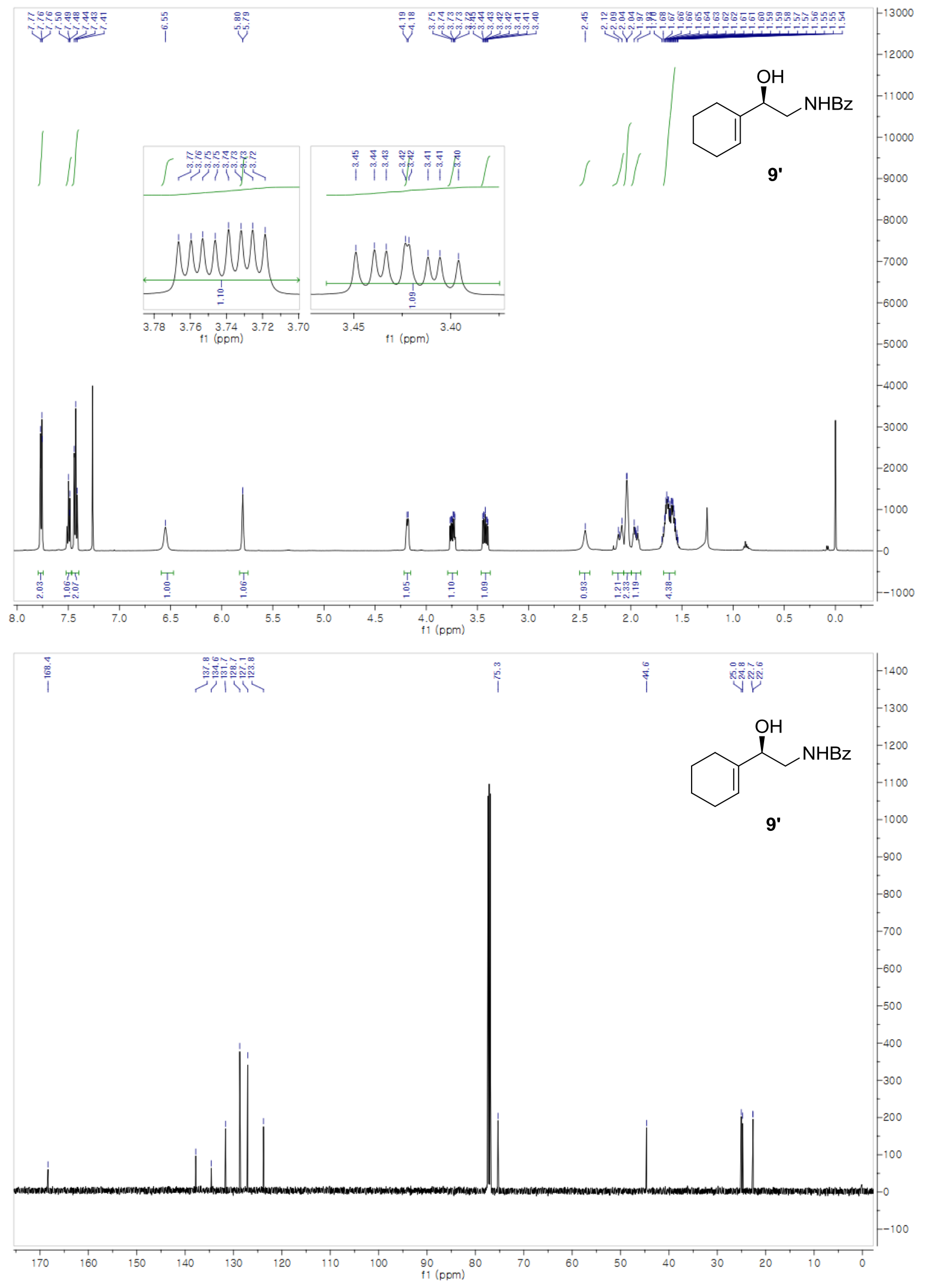




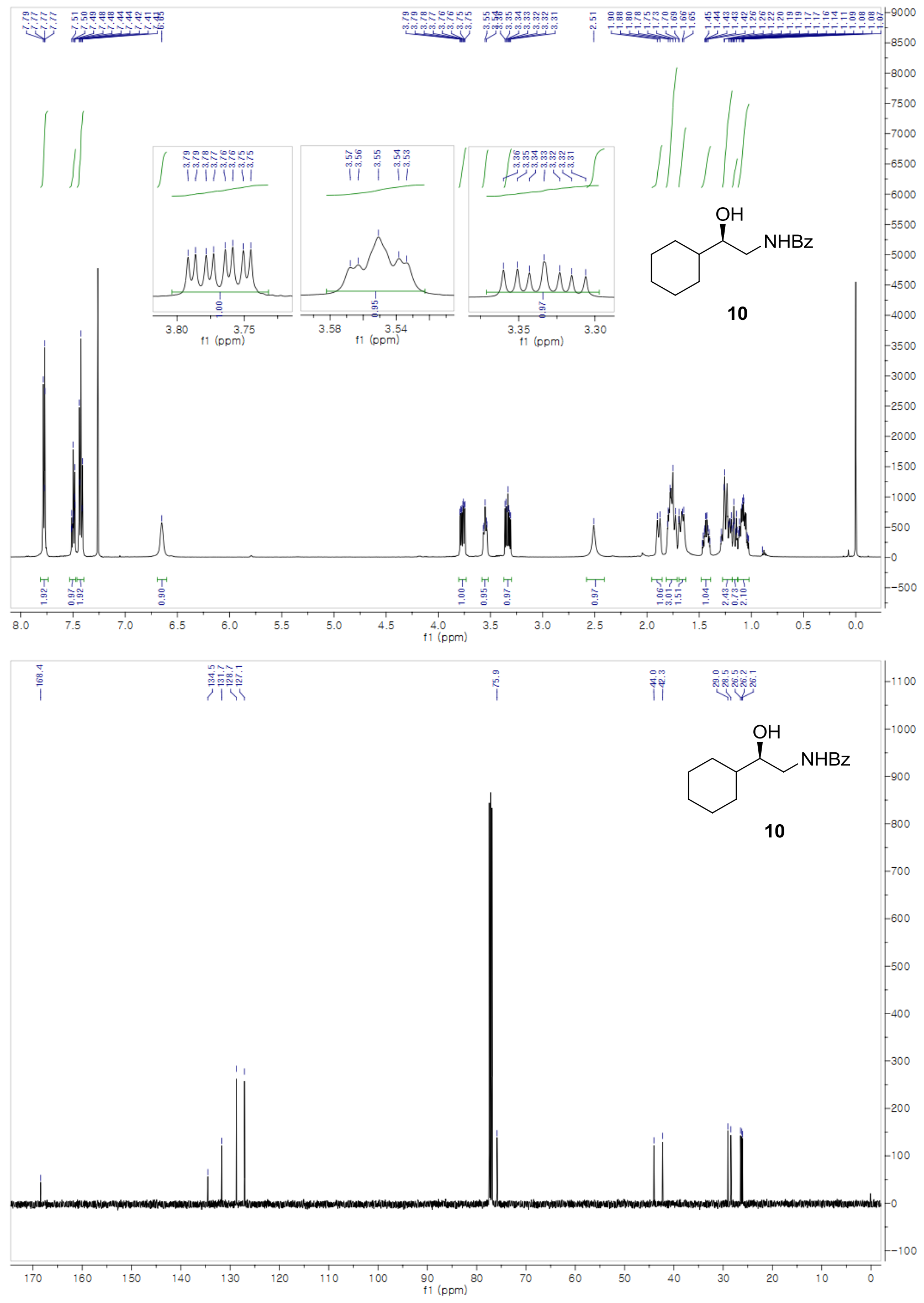




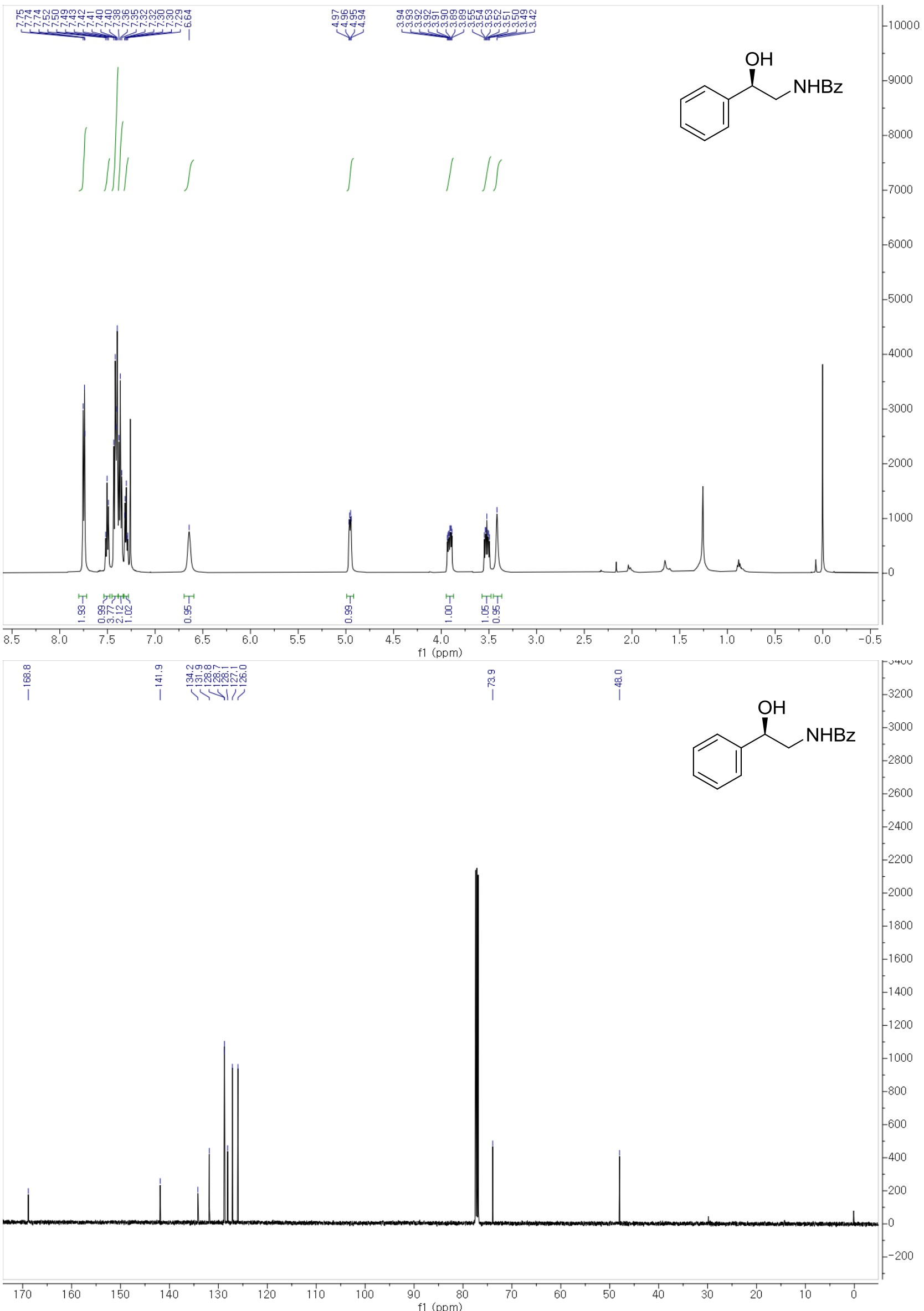




\section{HPLC Spectra}

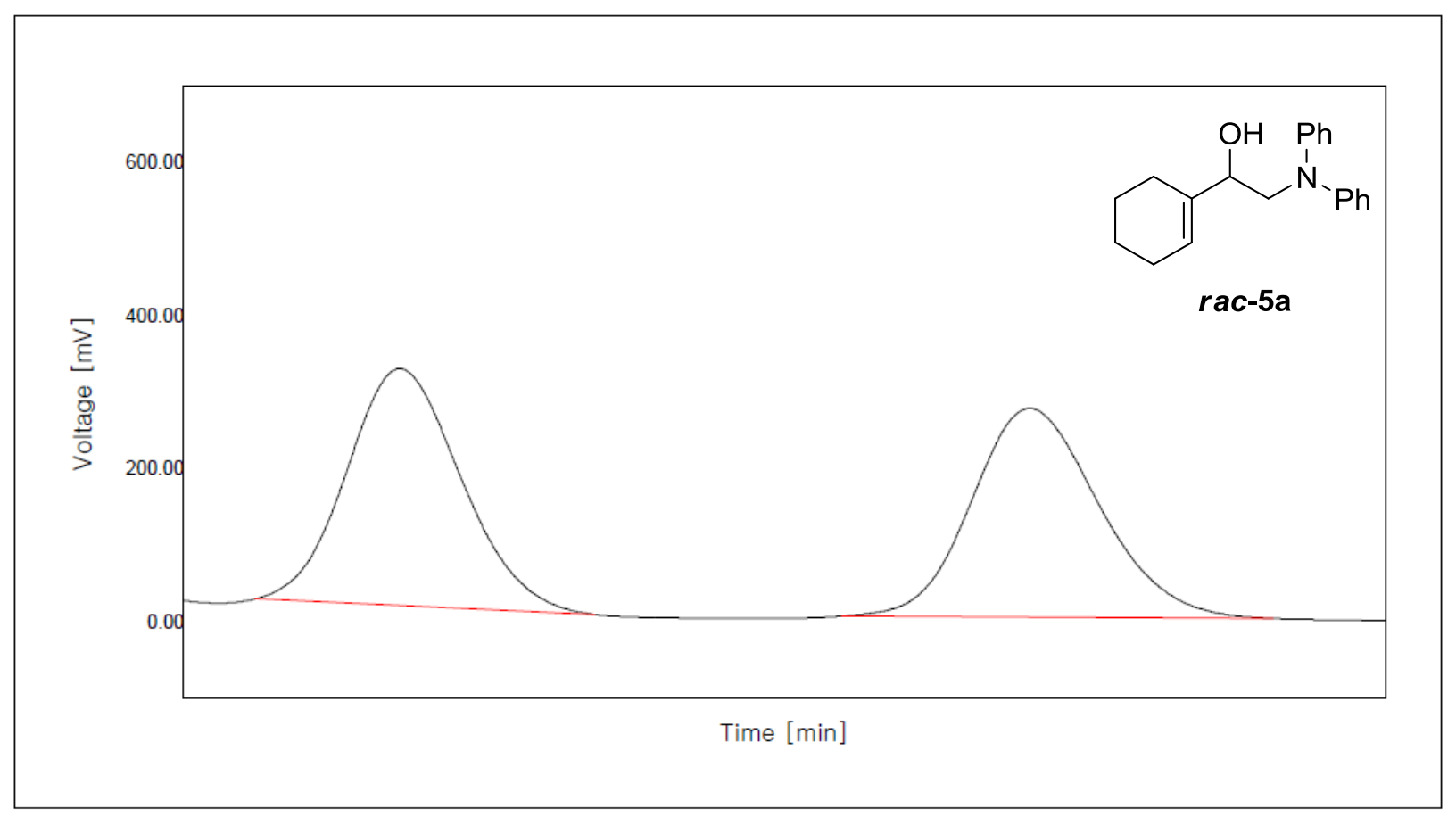

\begin{tabular}{|c|r|r|}
\hline No. & Time(min) & Area ratio (\%) \\
\hline 1 & 8.2067 & 50.47 \\
\hline 2 & 9.0967 & 49.53 \\
\hline
\end{tabular}

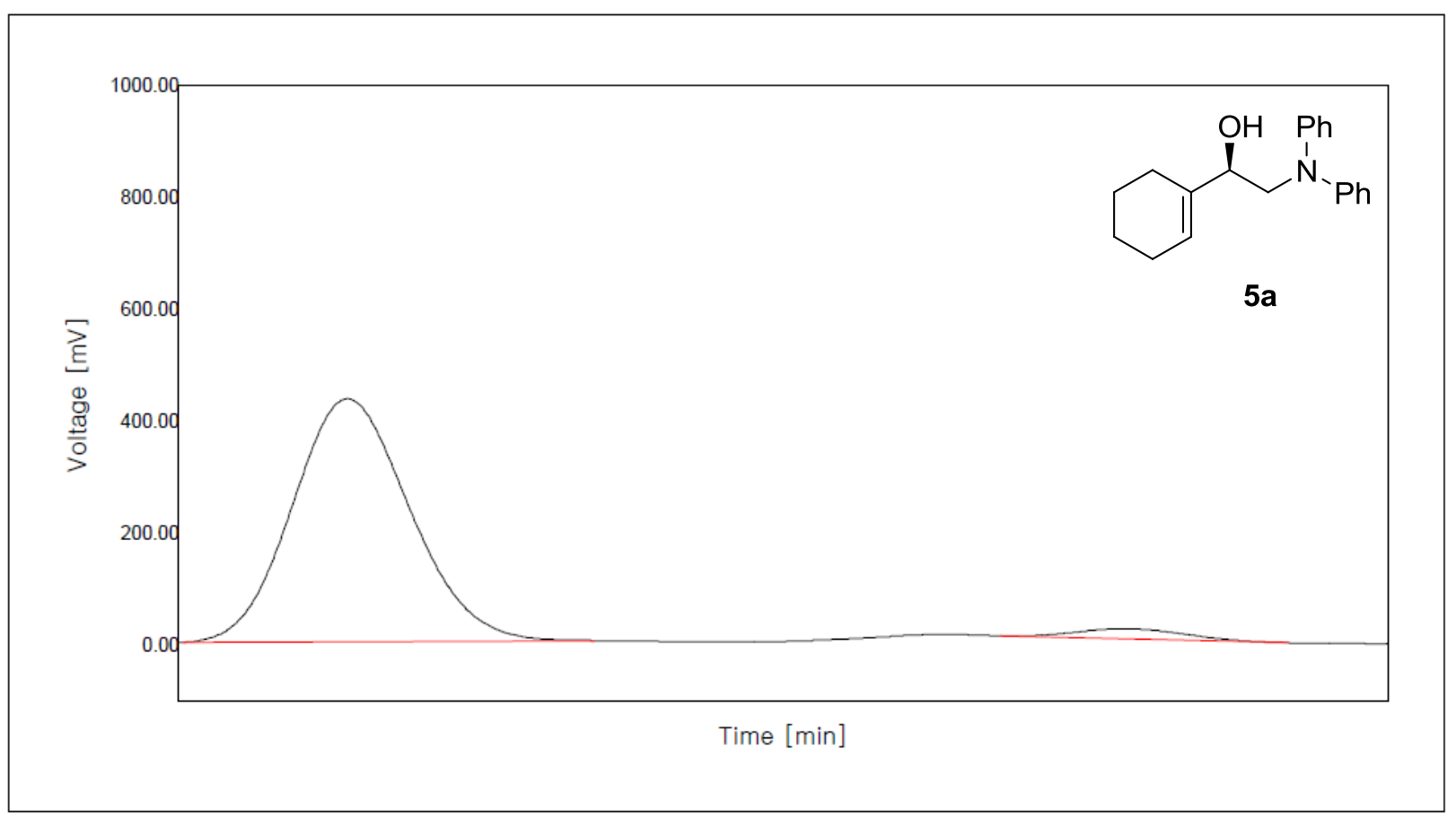

\begin{tabular}{|r|r|r|}
\hline No. & Time(min) & Area ratio (\%) \\
\hline 1 & 8.1950 & 96.24 \\
\hline 2 & 9.0950 & 3.76 \\
\hline
\end{tabular}




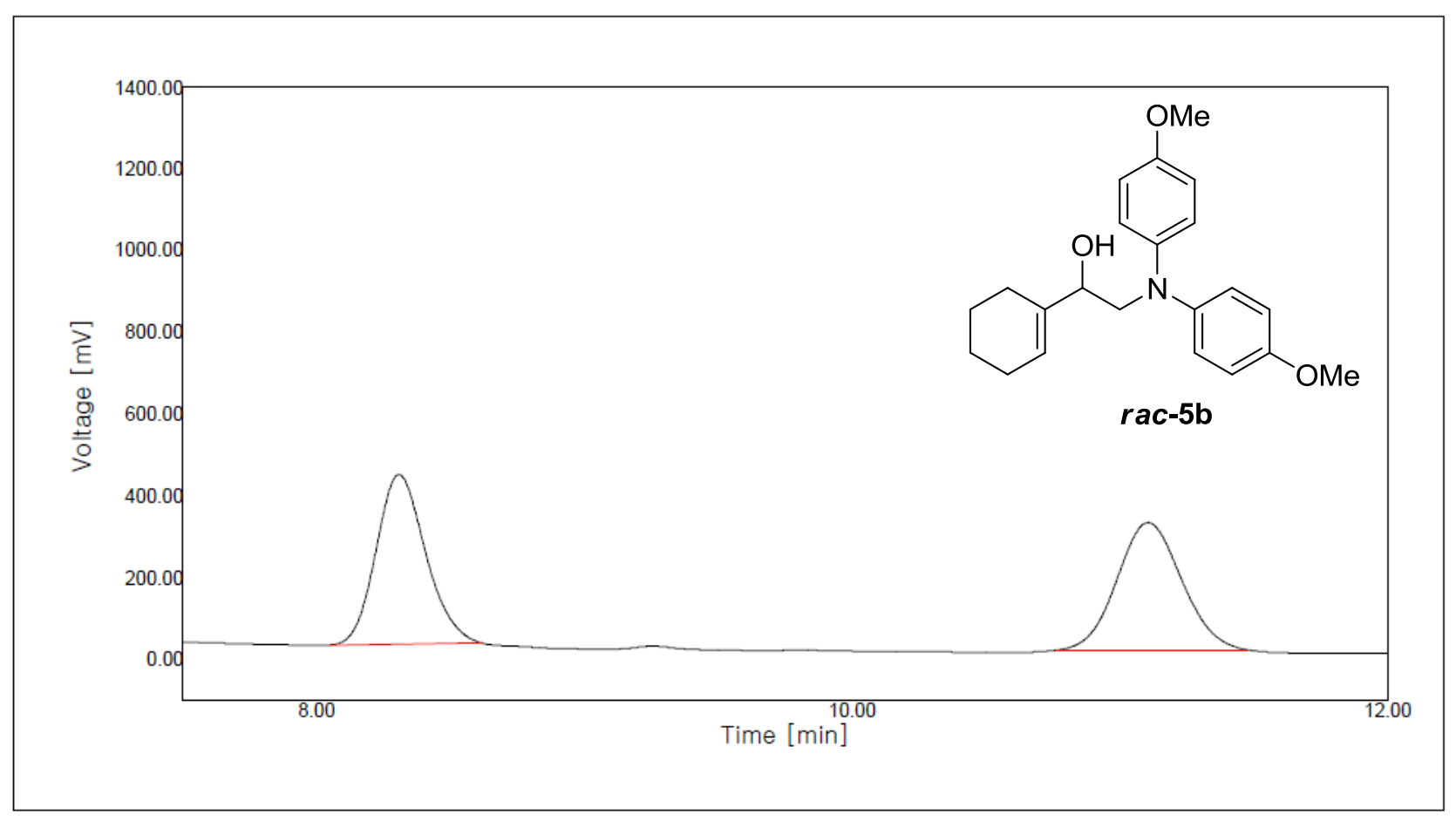

\begin{tabular}{|c|c|c|}
\hline No. & Time(min) & Area ratio (\%) \\
\hline 1 & 8.3067 & 50.46 \\
\hline 2 & 11.1067 & 49.54 \\
\hline
\end{tabular}

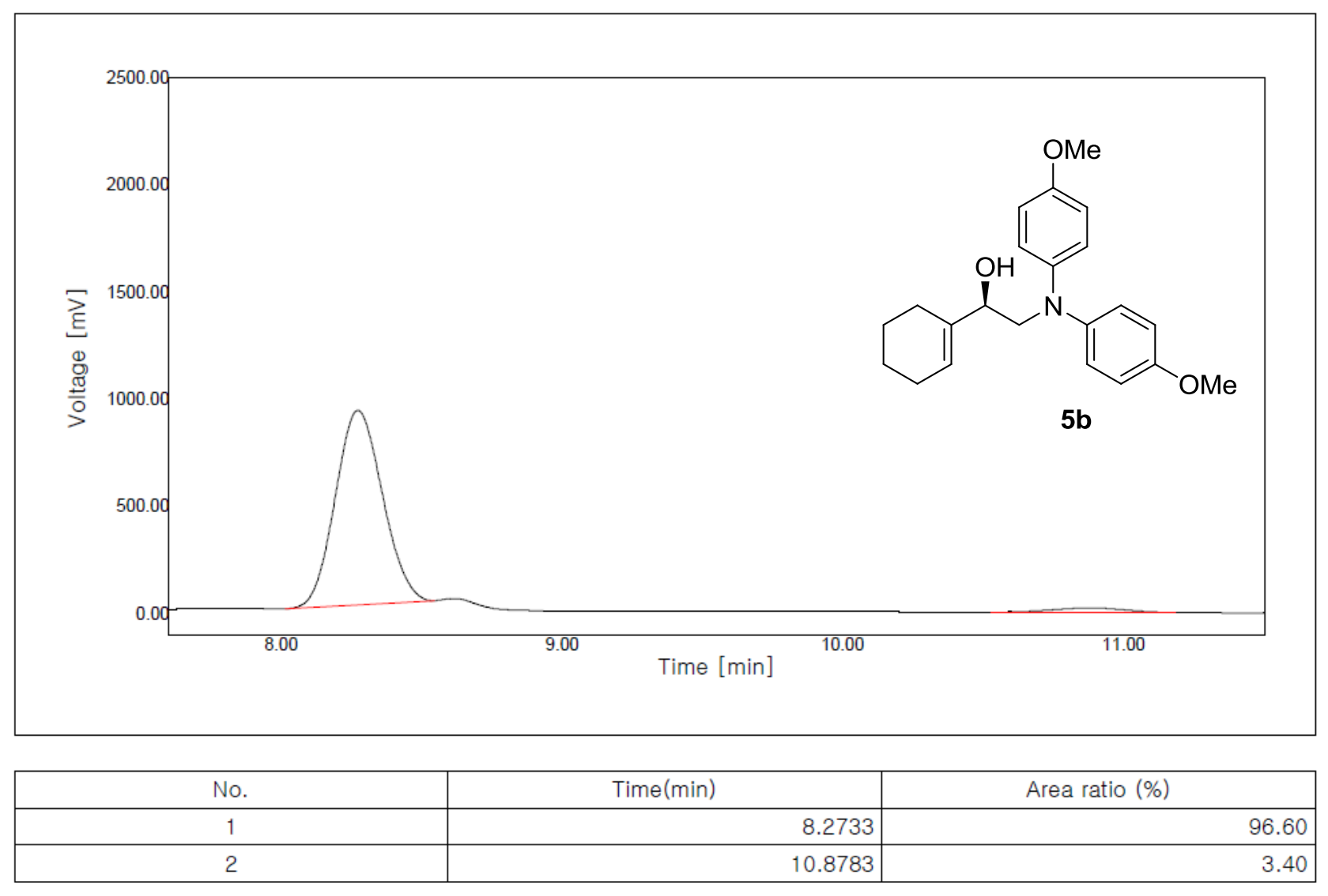




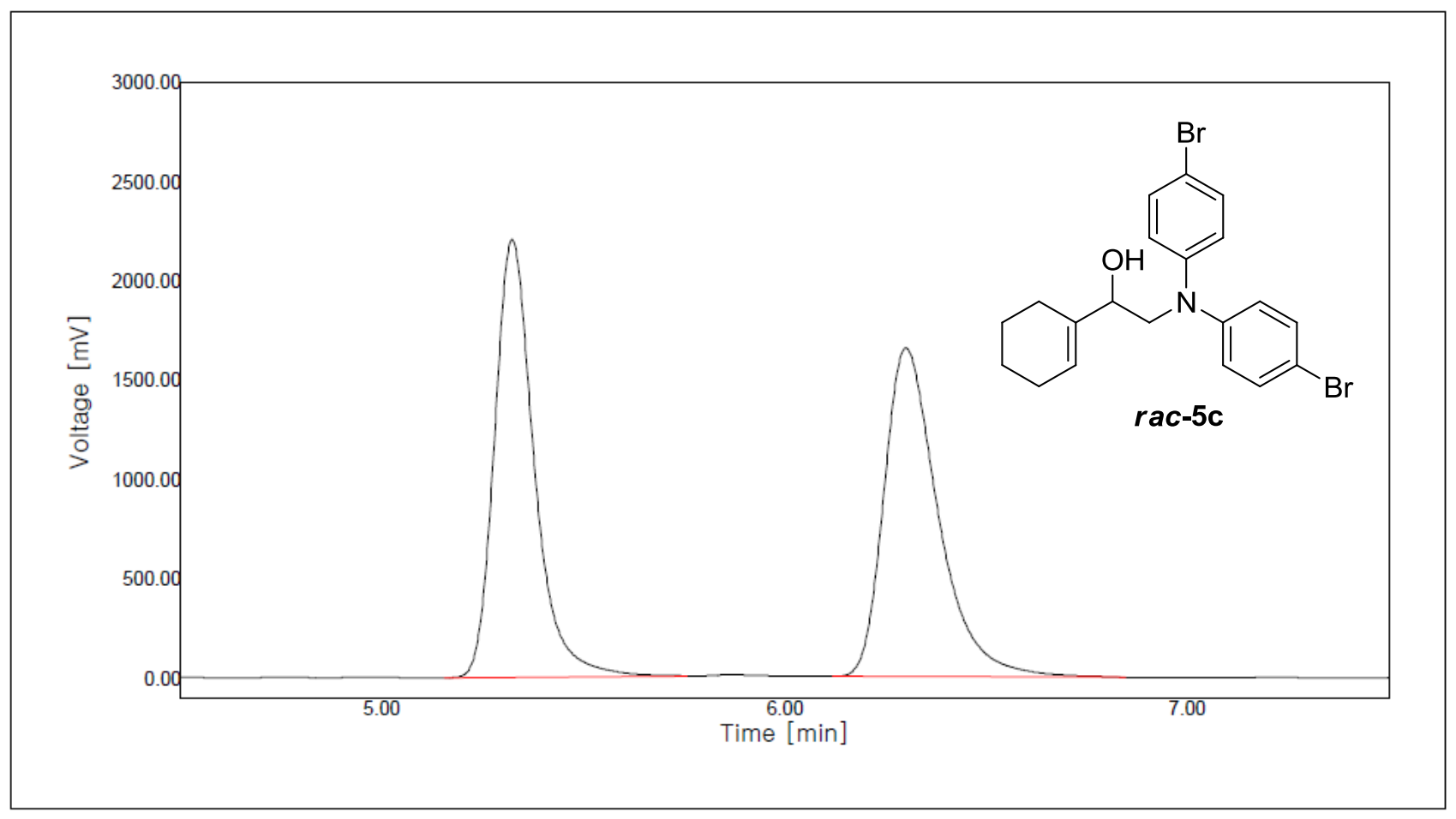

\begin{tabular}{|r|r|r|}
\hline No. & Time(min) & \multicolumn{1}{|c|}{ Area ratio (\%) } \\
\hline 1 & & 5.3233 \\
\hline 2 & 6.3017 & 50.32 \\
\hline
\end{tabular}

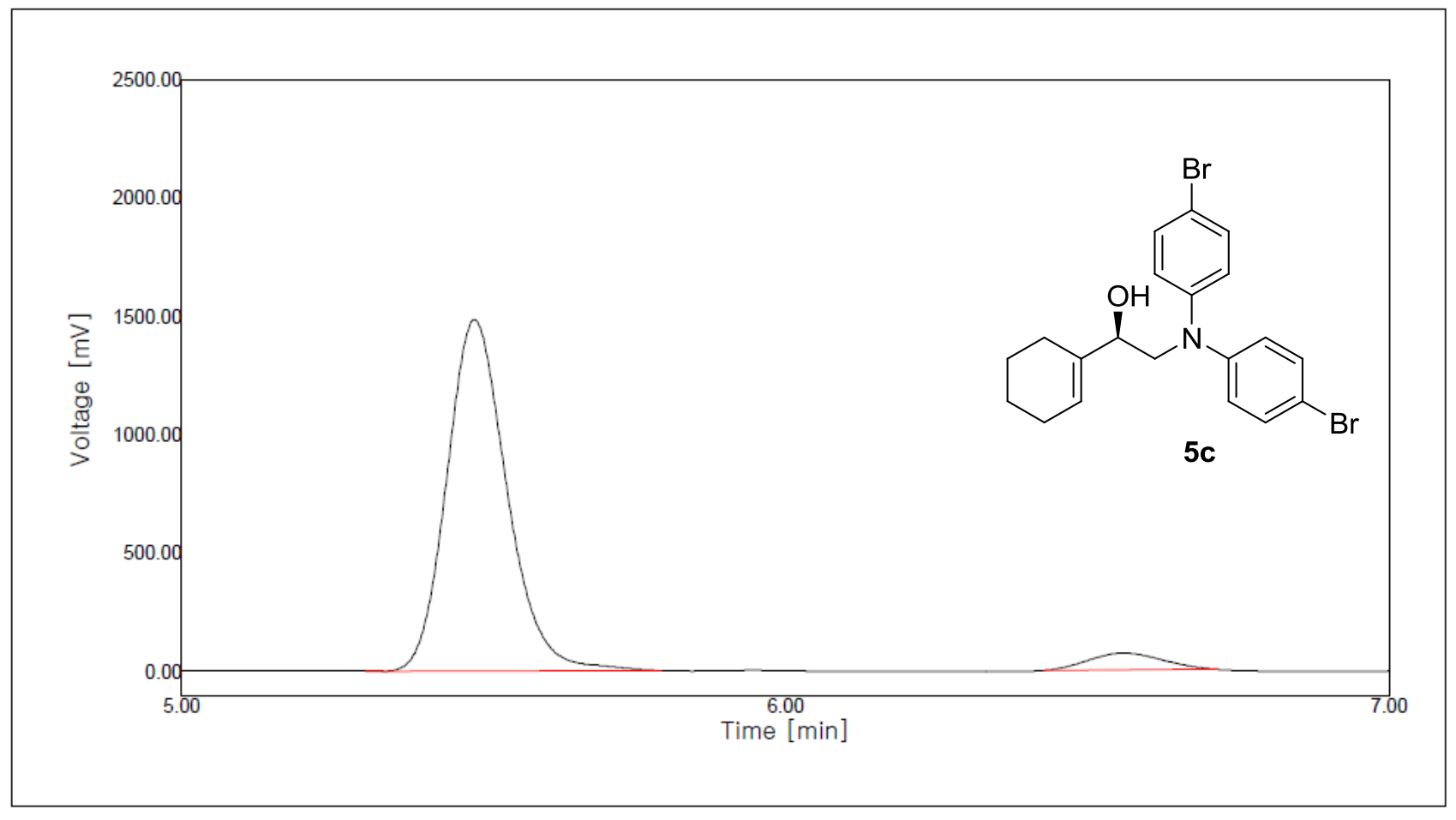

\begin{tabular}{|r|r|r|}
\hline No. & Time $(\mathrm{min})$ & \multicolumn{1}{|c|}{ Area ratio (\%) } \\
\hline 1 & & 94.43 \\
\hline 2 & 5.4850 & 5.57 \\
\hline
\end{tabular}




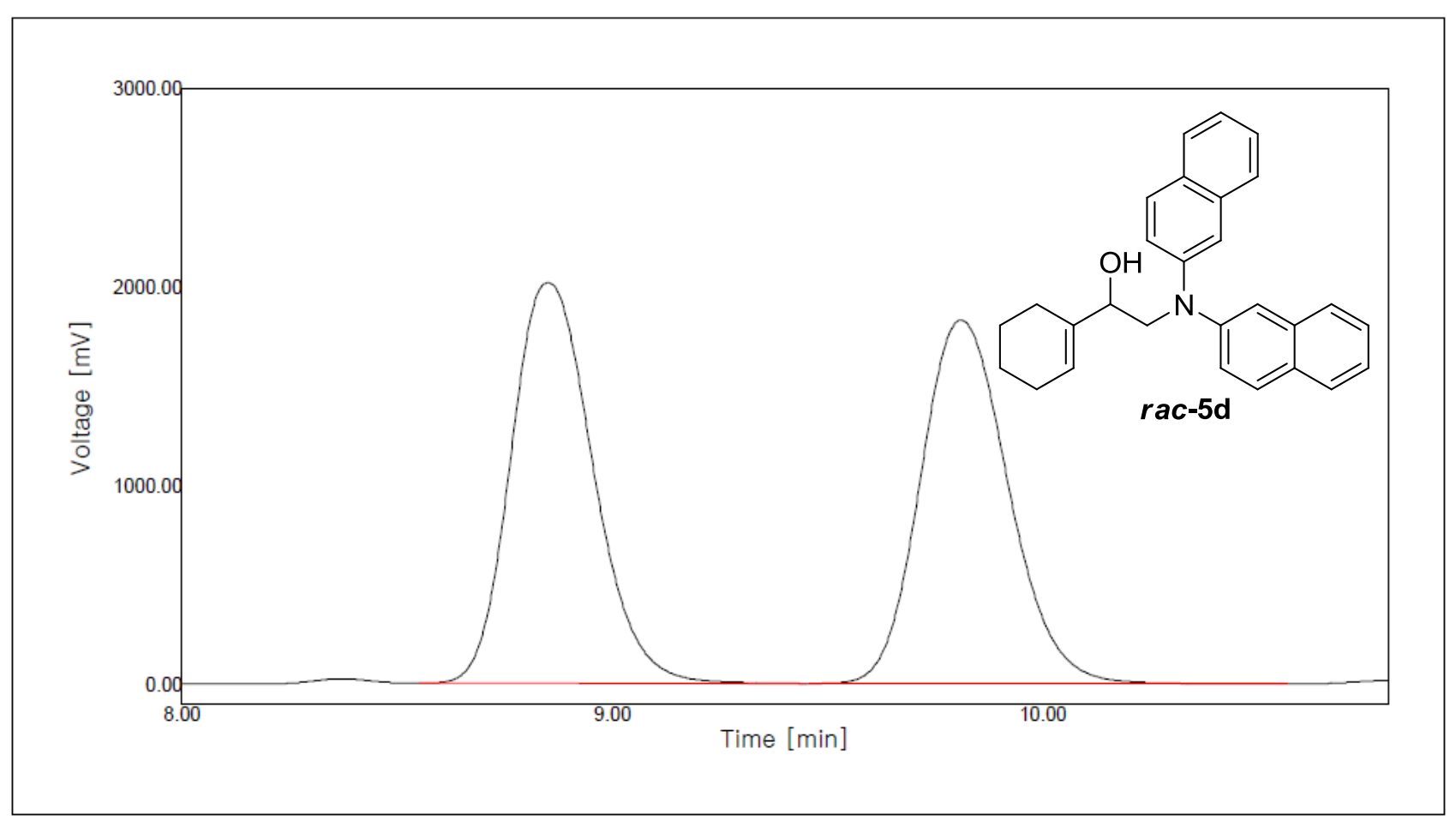

\begin{tabular}{|r|r|r|}
\hline No. & Time $(\mathrm{min})$ & \multicolumn{1}{|c|}{ Area ratio (\%) } \\
\hline 1 & & 8.8500 \\
\hline 2 & 9.8083 & 50.68 \\
\hline
\end{tabular}

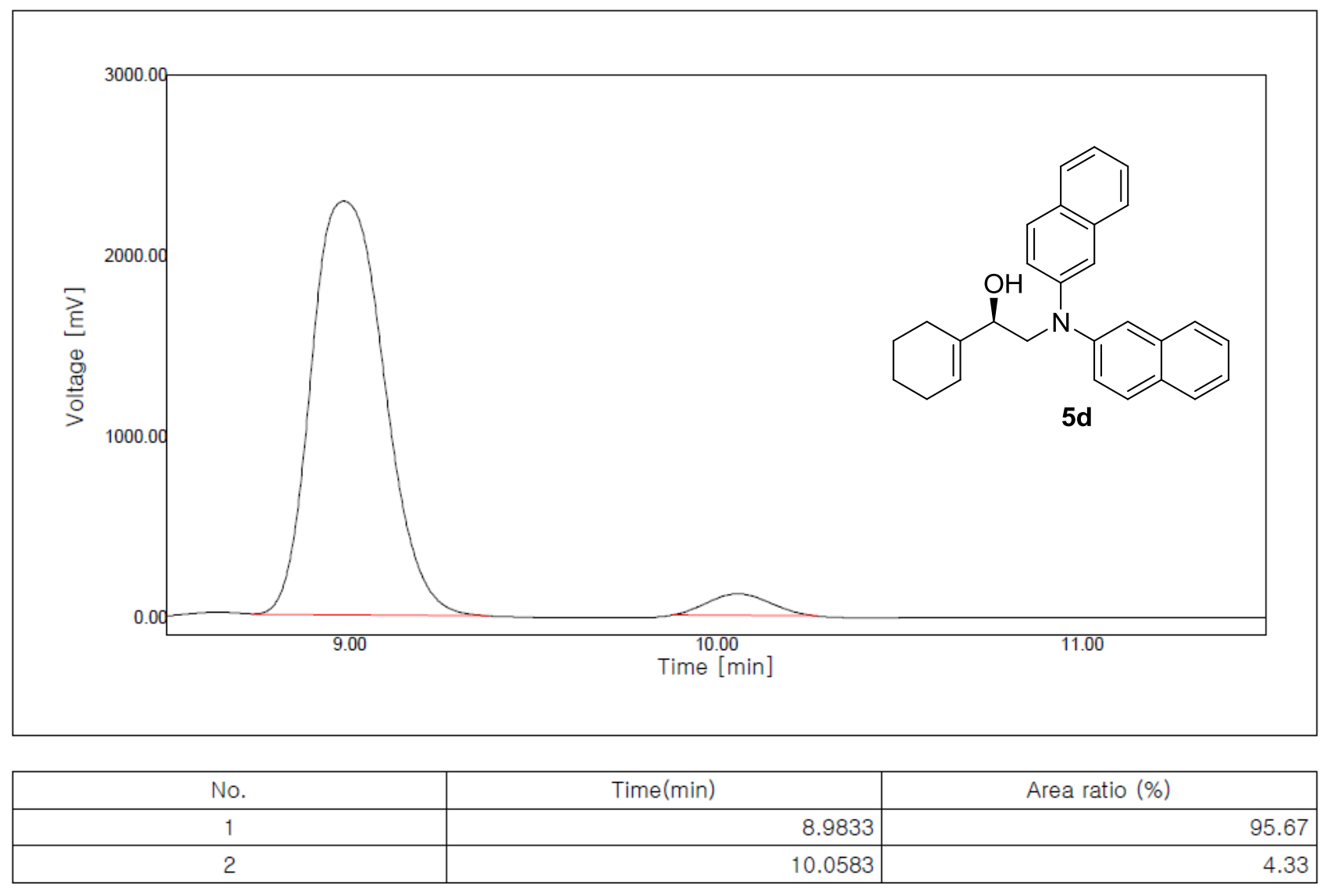




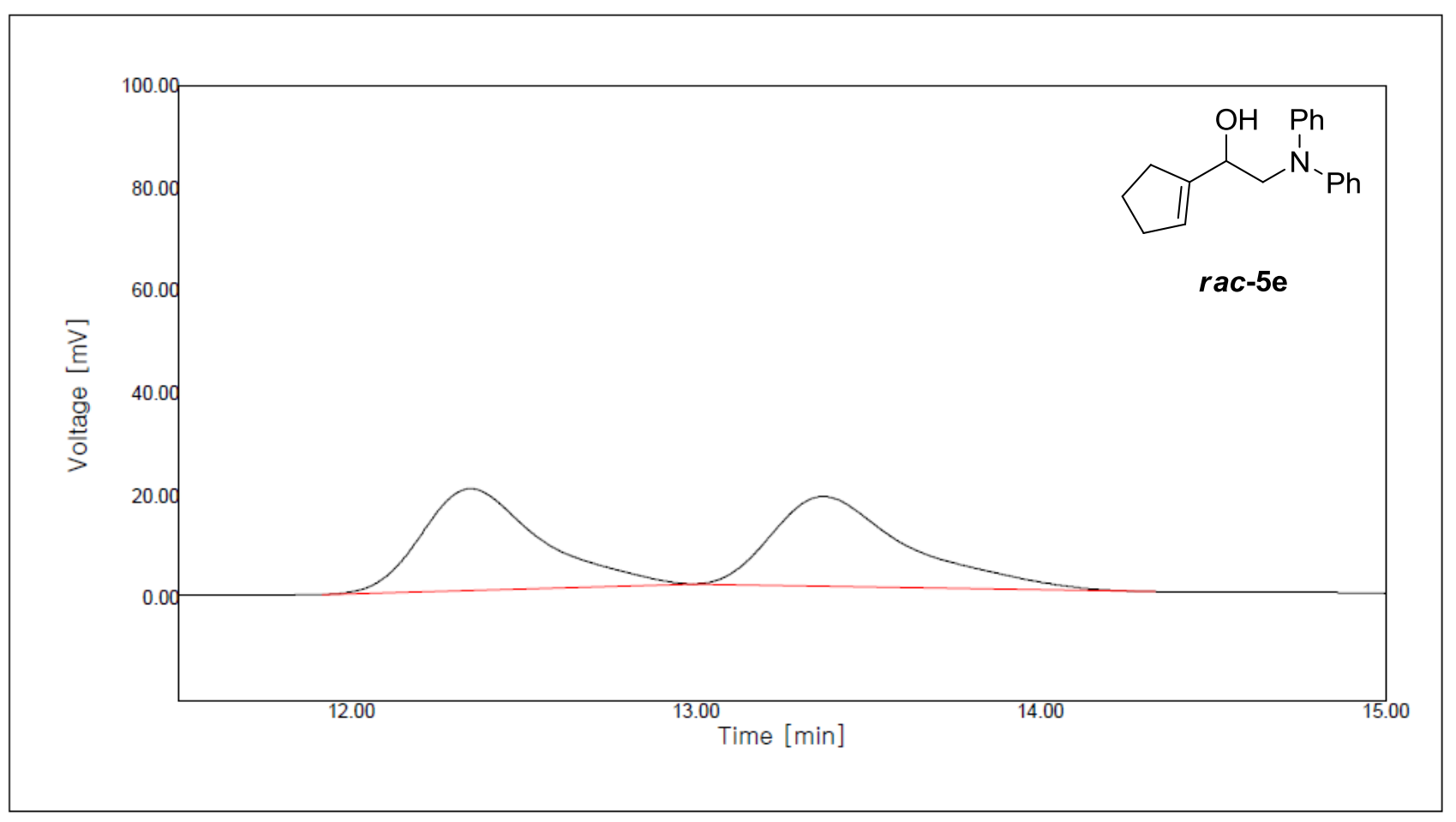

\begin{tabular}{|r|r|r|}
\hline No. & Time $(\mathrm{min})$ & \multicolumn{1}{|c|}{ Area ratio (\%) } \\
\hline 1 & & 12.3450 \\
\hline 2 & 13.3700 & 59.78 \\
\hline
\end{tabular}

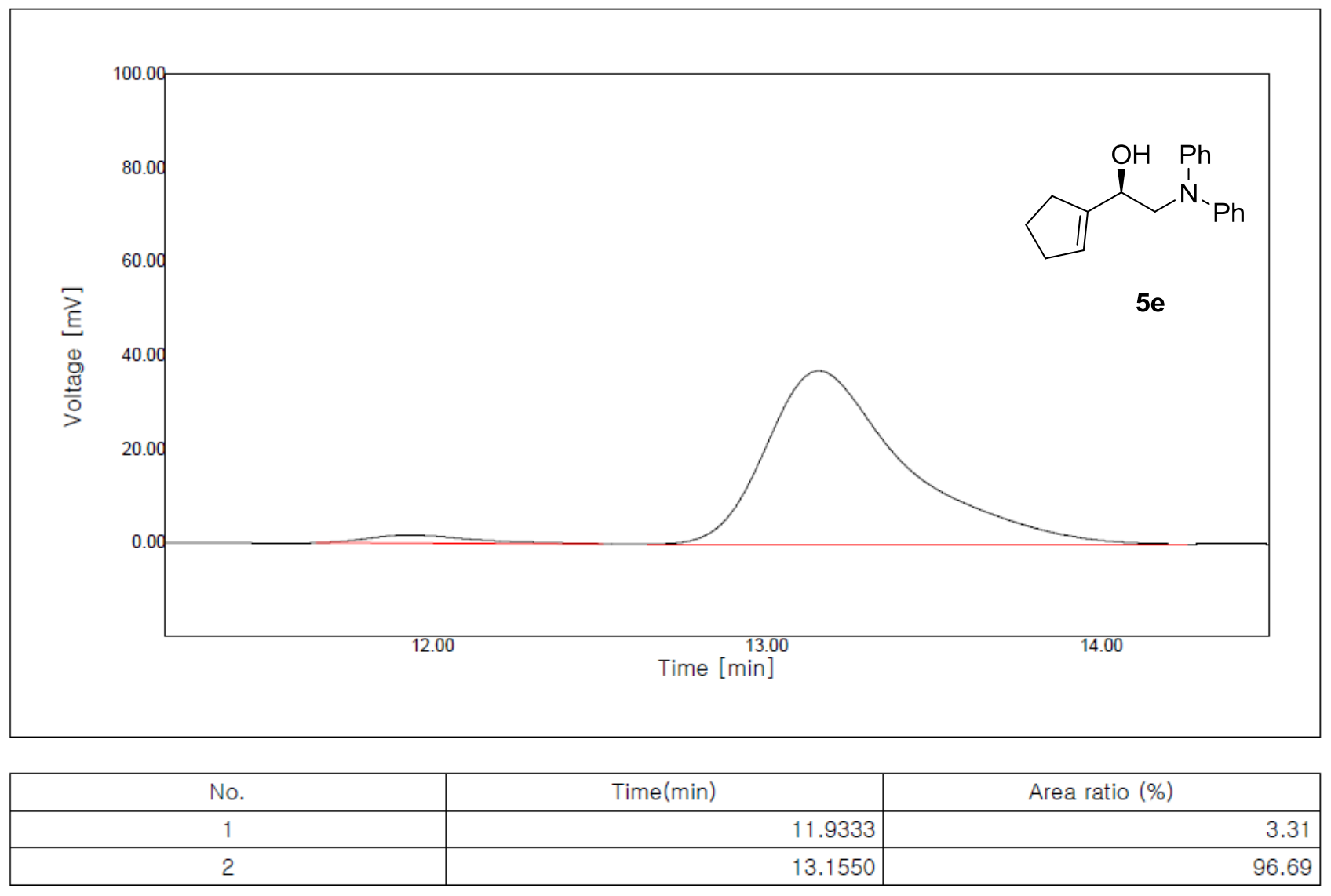




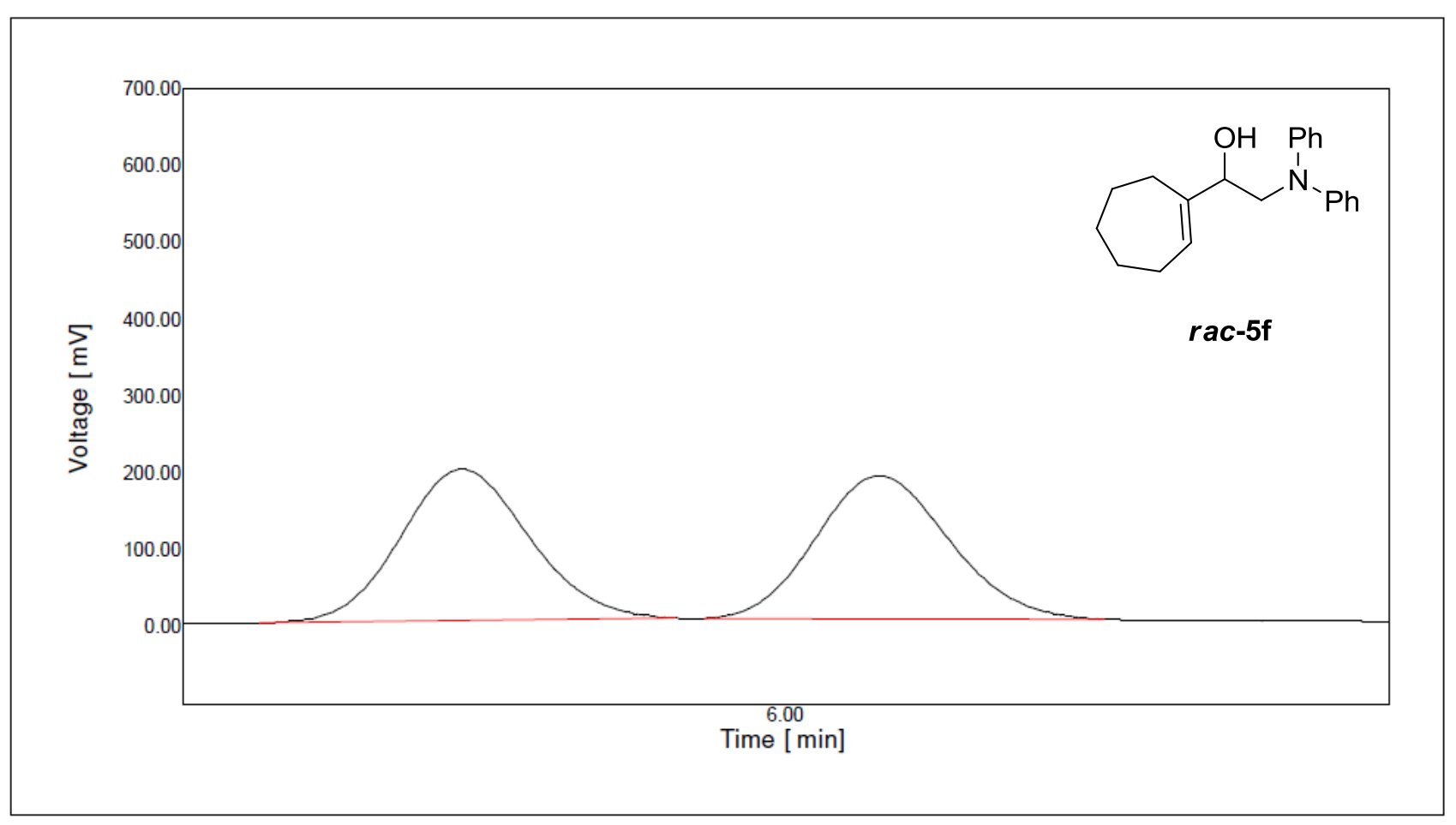

\begin{tabular}{|c|c|c|}
\hline No. & Time $(\min )$ & Area ratio $(\%)$ \\
\hline 1 & 5.7850 & 50.42 \\
\hline 2 & 6.0617 & 49.58 \\
\hline
\end{tabular}

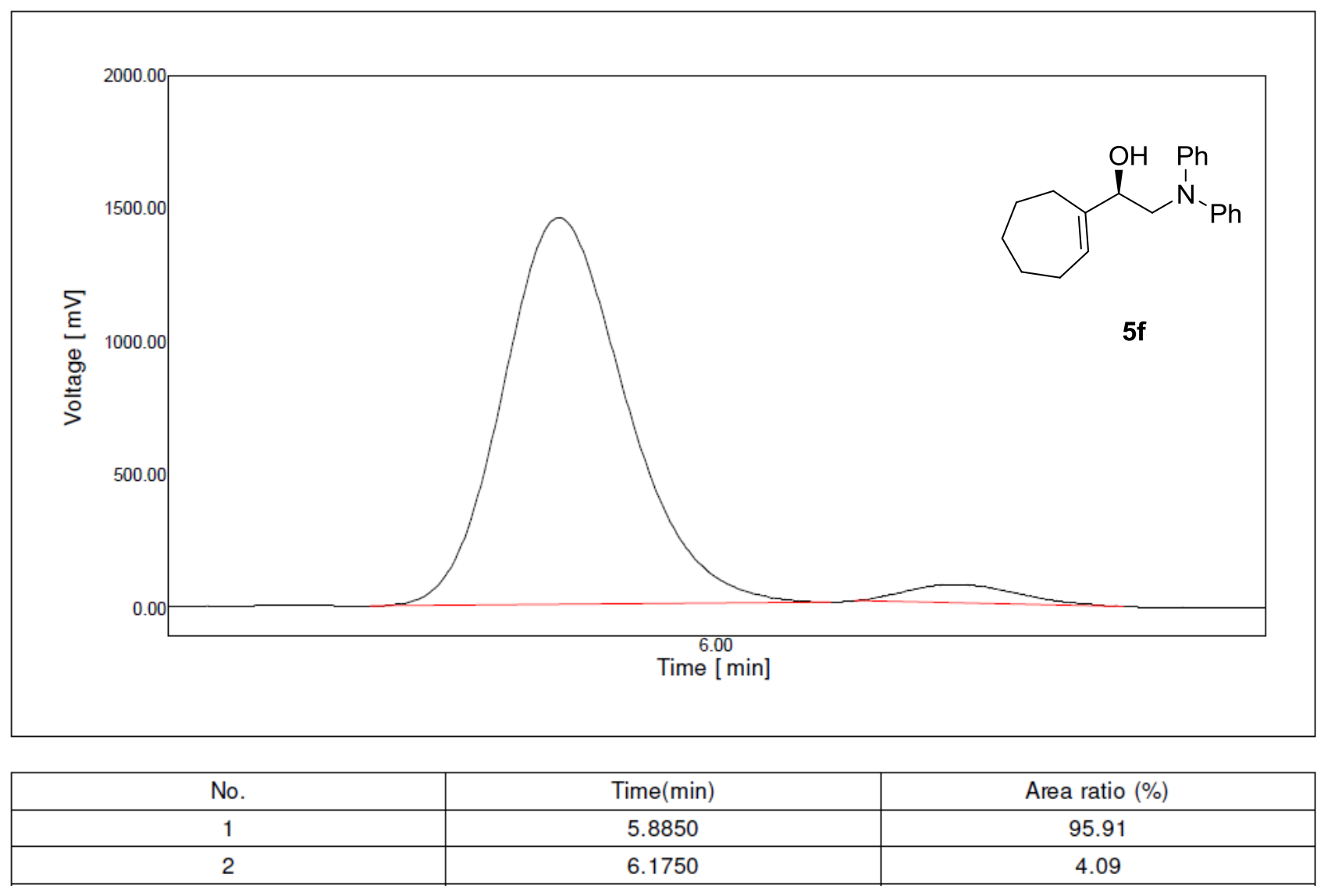




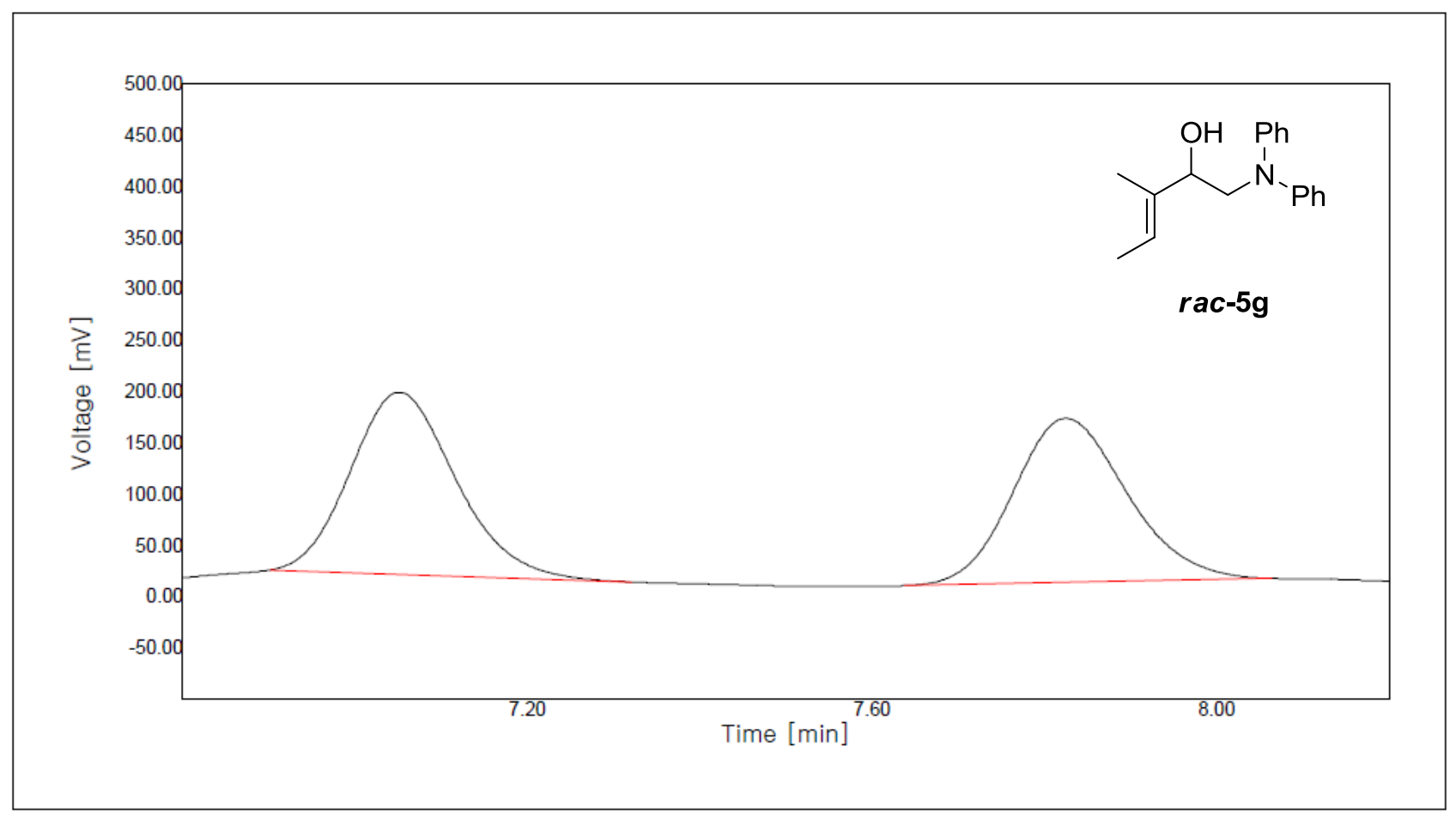

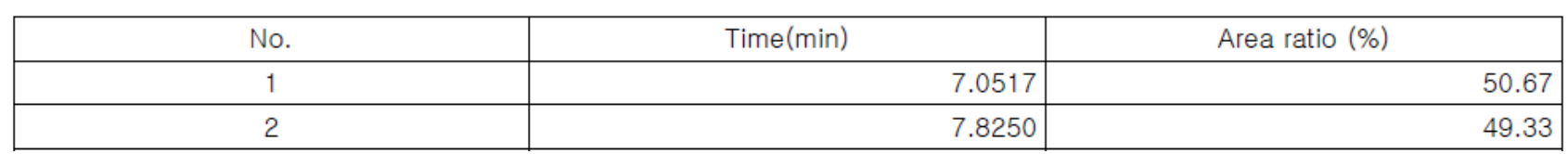

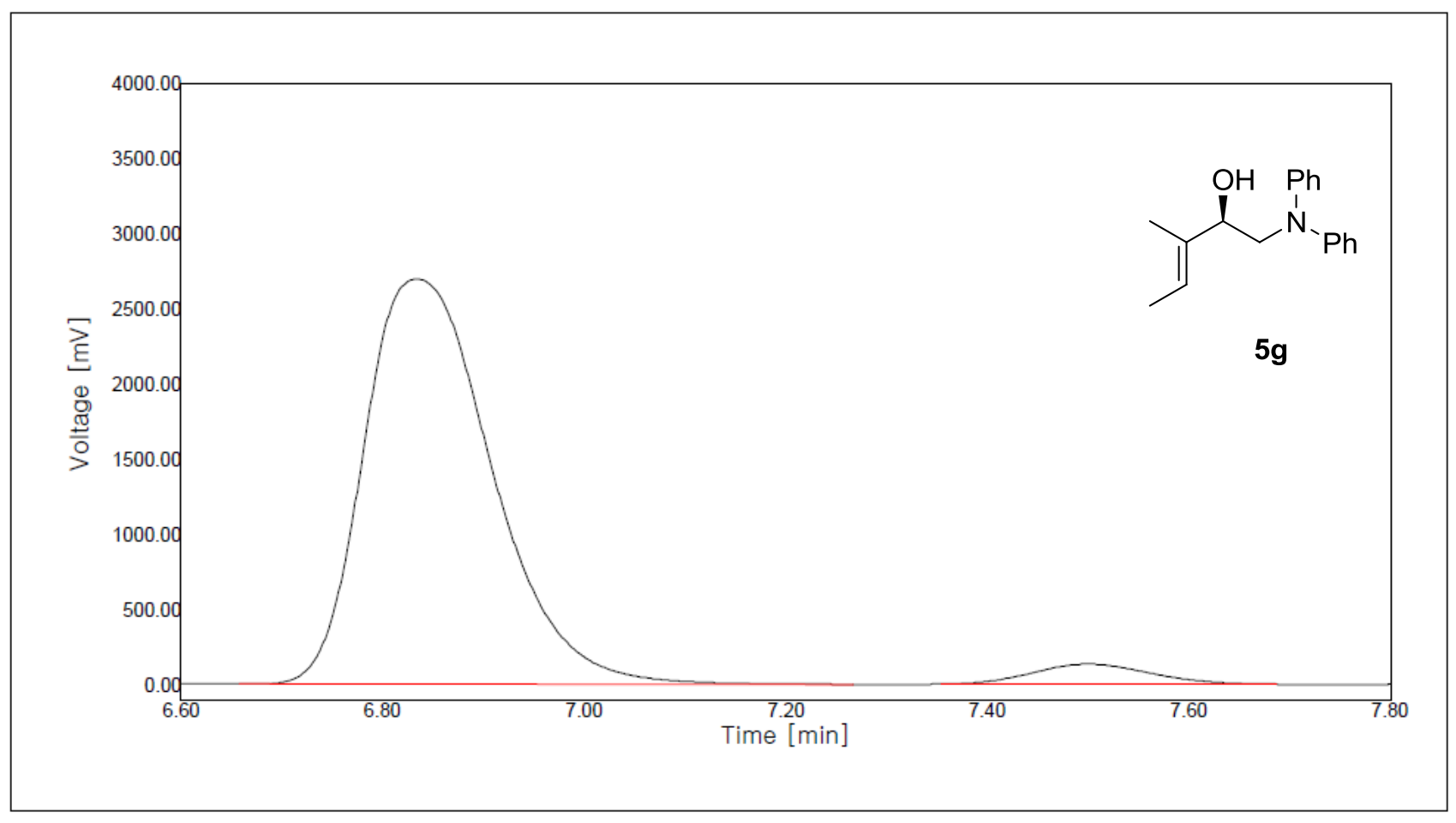

\begin{tabular}{|r|r|r|}
\hline No. & Time $(m i n)$ & \multicolumn{1}{|c|}{ Area ratio (\%) } \\
\hline 1 & & 6.8333 \\
\hline 2 & 7.5000 & 4.36 \\
\hline
\end{tabular}




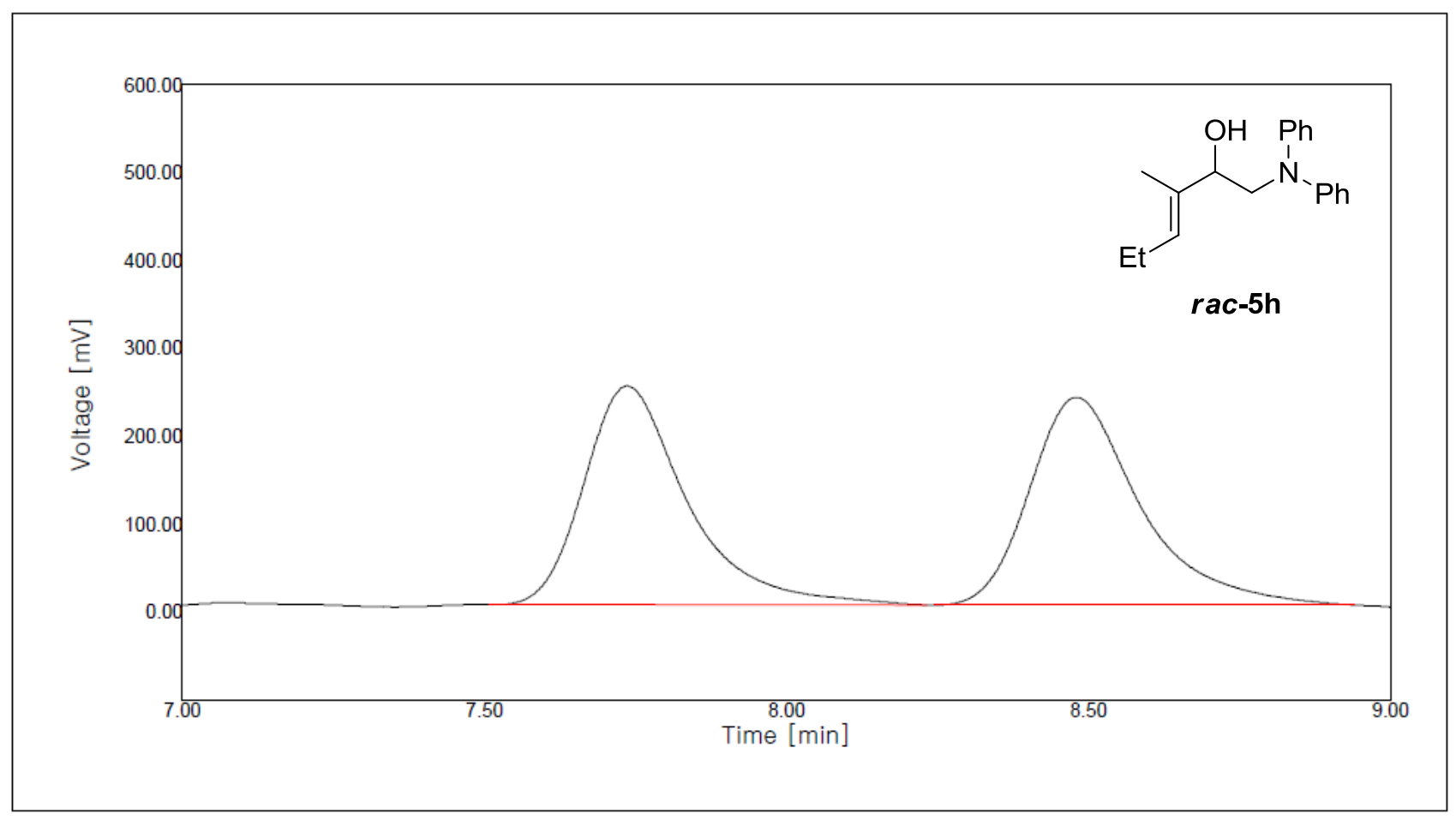

\begin{tabular}{|r|r|r|}
\hline No. & Time $(\min )$ & \multicolumn{1}{|c|}{ Area ratio (\%) } \\
\hline 1 & & 7.7367 \\
\hline 2 & 8.4800 & 59.61 \\
\hline
\end{tabular}

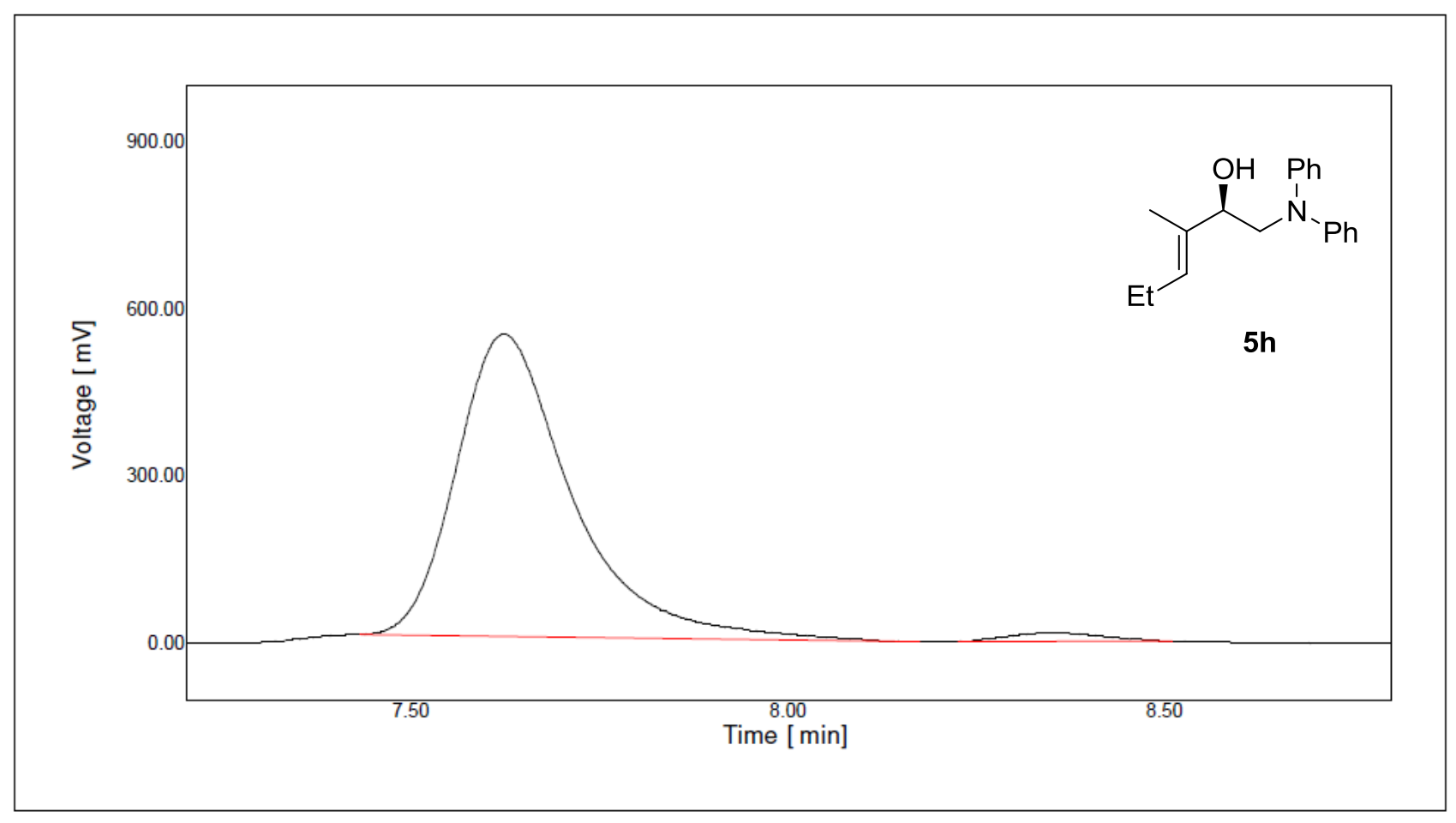

\begin{tabular}{|c|c|c|}
\hline No. & Time $(\mathrm{min})$ & Area ratio (\%) \\
\hline 1 & 7.6233 & 97.83 \\
\hline 2 & 8.3517 & 2.17 \\
\hline
\end{tabular}




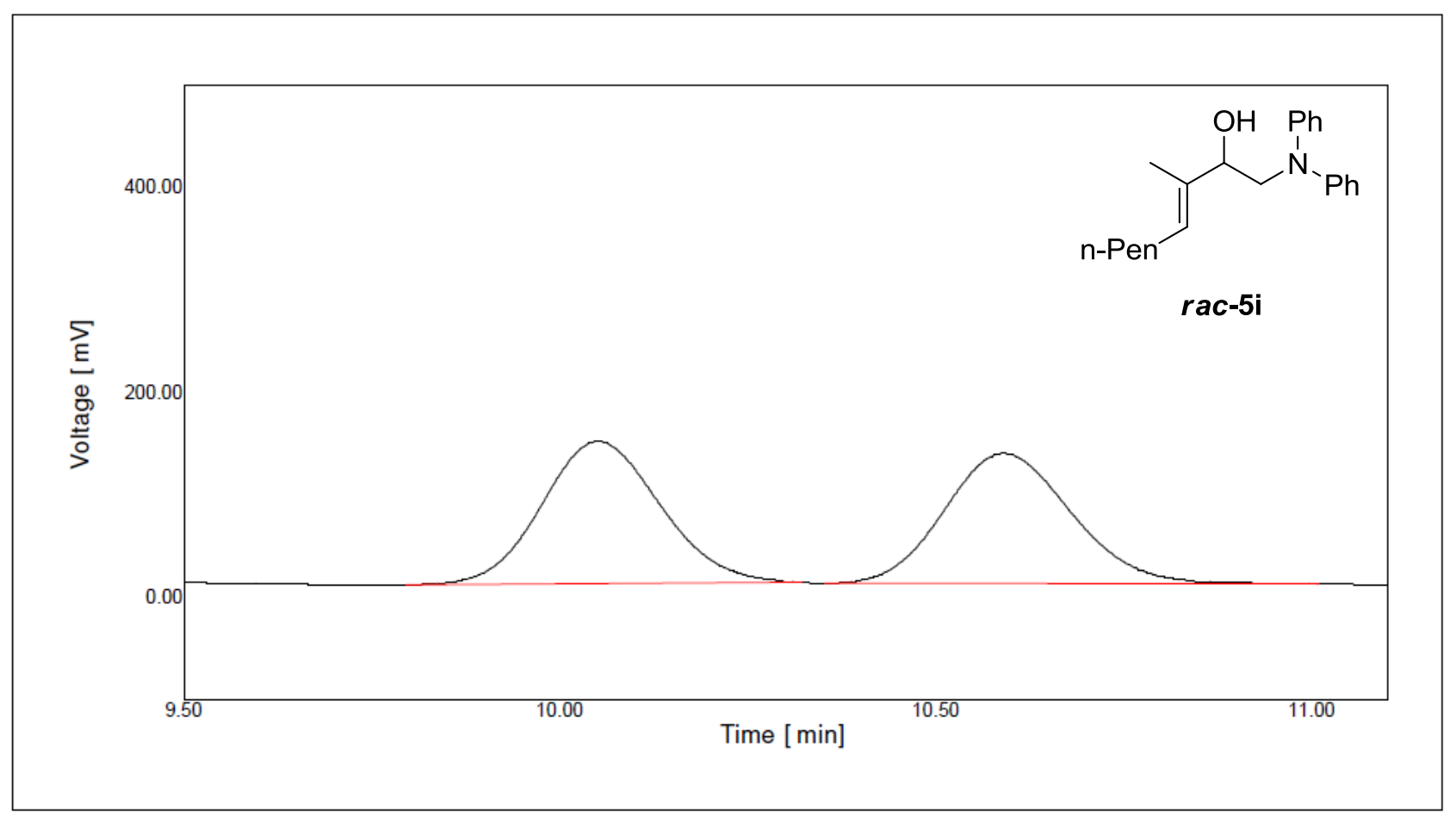

\begin{tabular}{|c|c|c|}
\hline No. & Time $(\mathrm{min})$ & Area ratio $(\%)$ \\
\hline 1 & 10.0517 & 50.62 \\
\hline 2 & 10.5900 & 49.38 \\
\hline
\end{tabular}

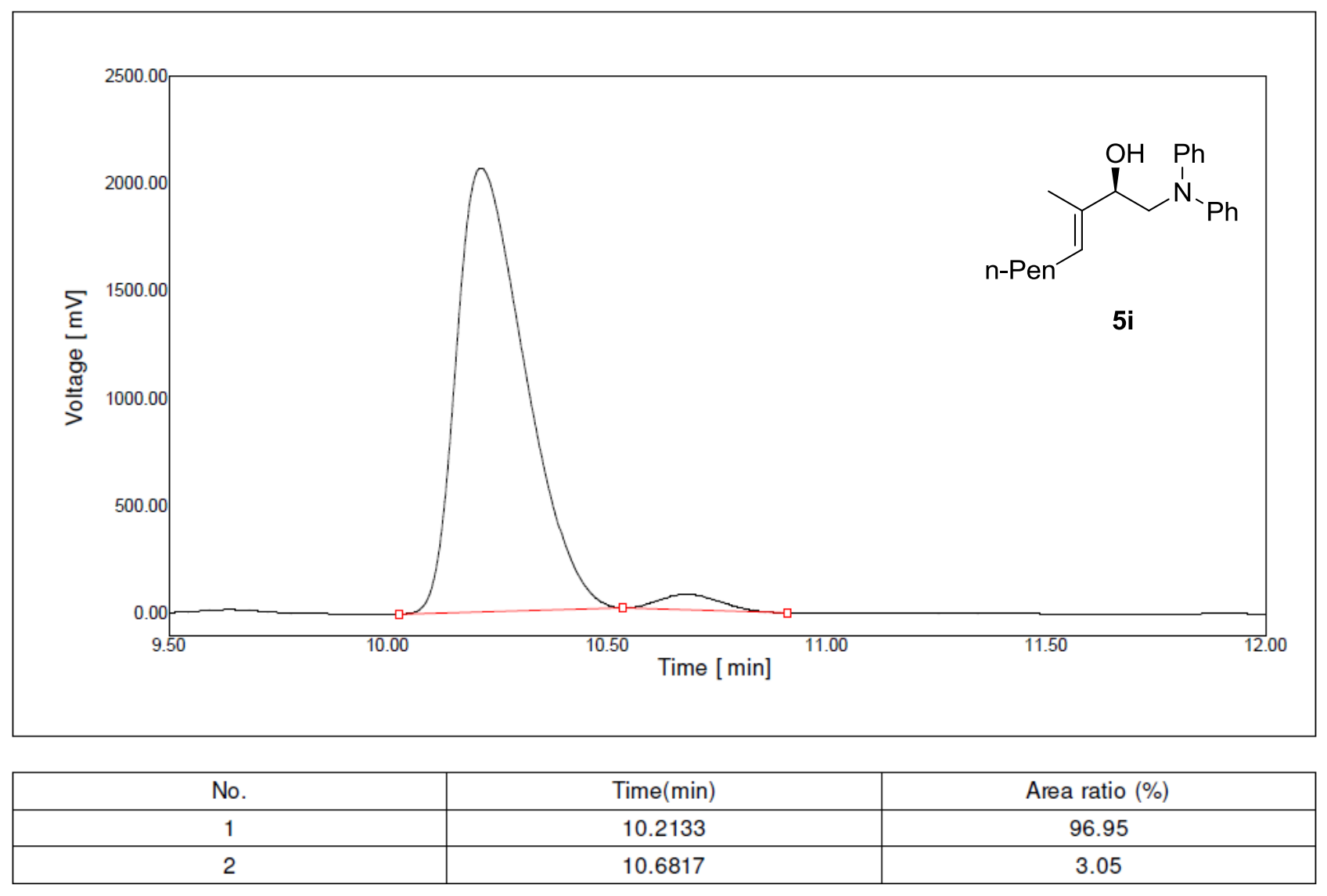




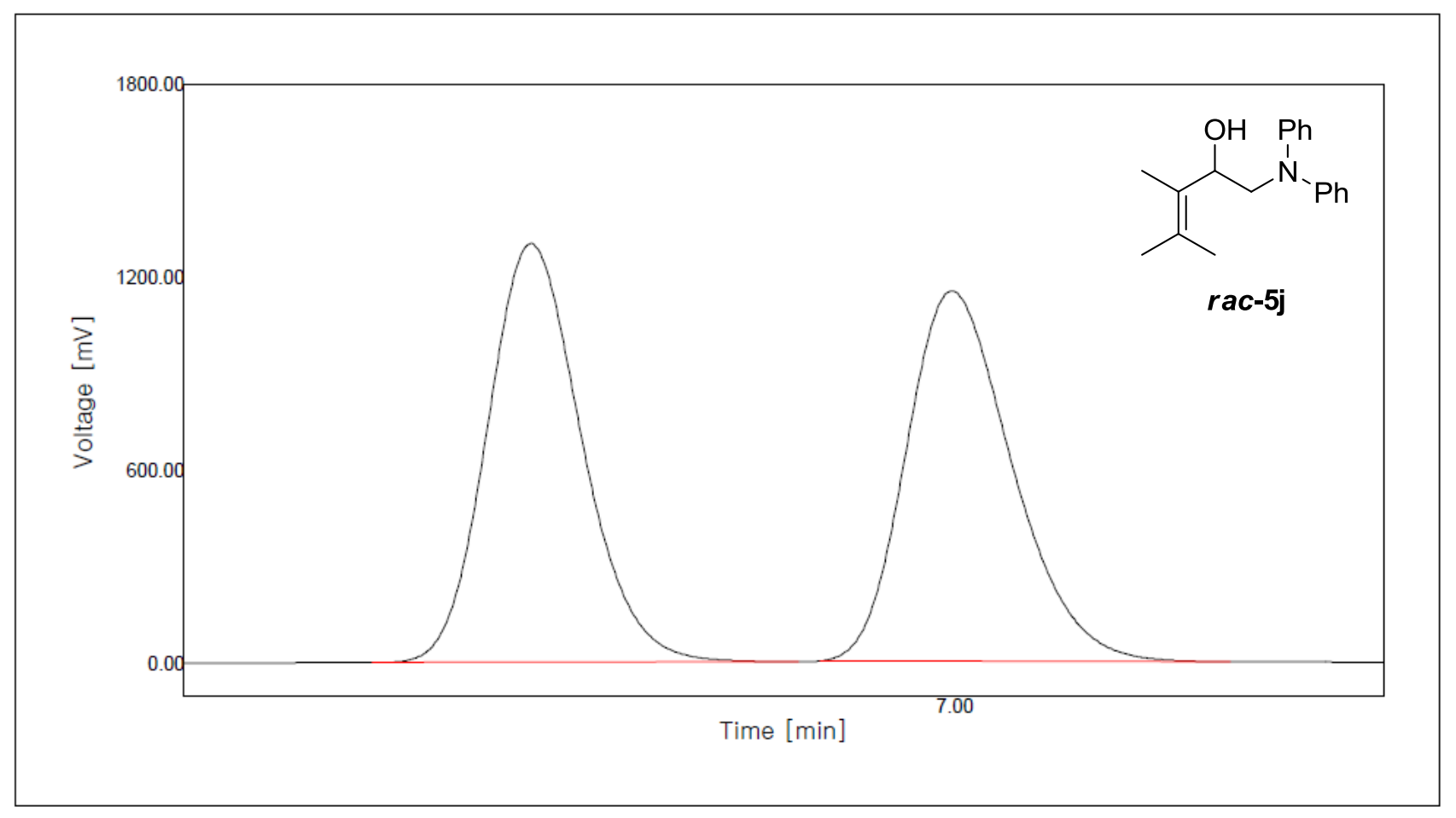

\begin{tabular}{|r|r|r|}
\hline No. & Time $(\mathrm{min})$ & \multicolumn{1}{|c|}{ Area ratio (\%) } \\
\hline 1 & & 6.5050 \\
\hline 2 & 6.9967 & 40.26 \\
\hline
\end{tabular}

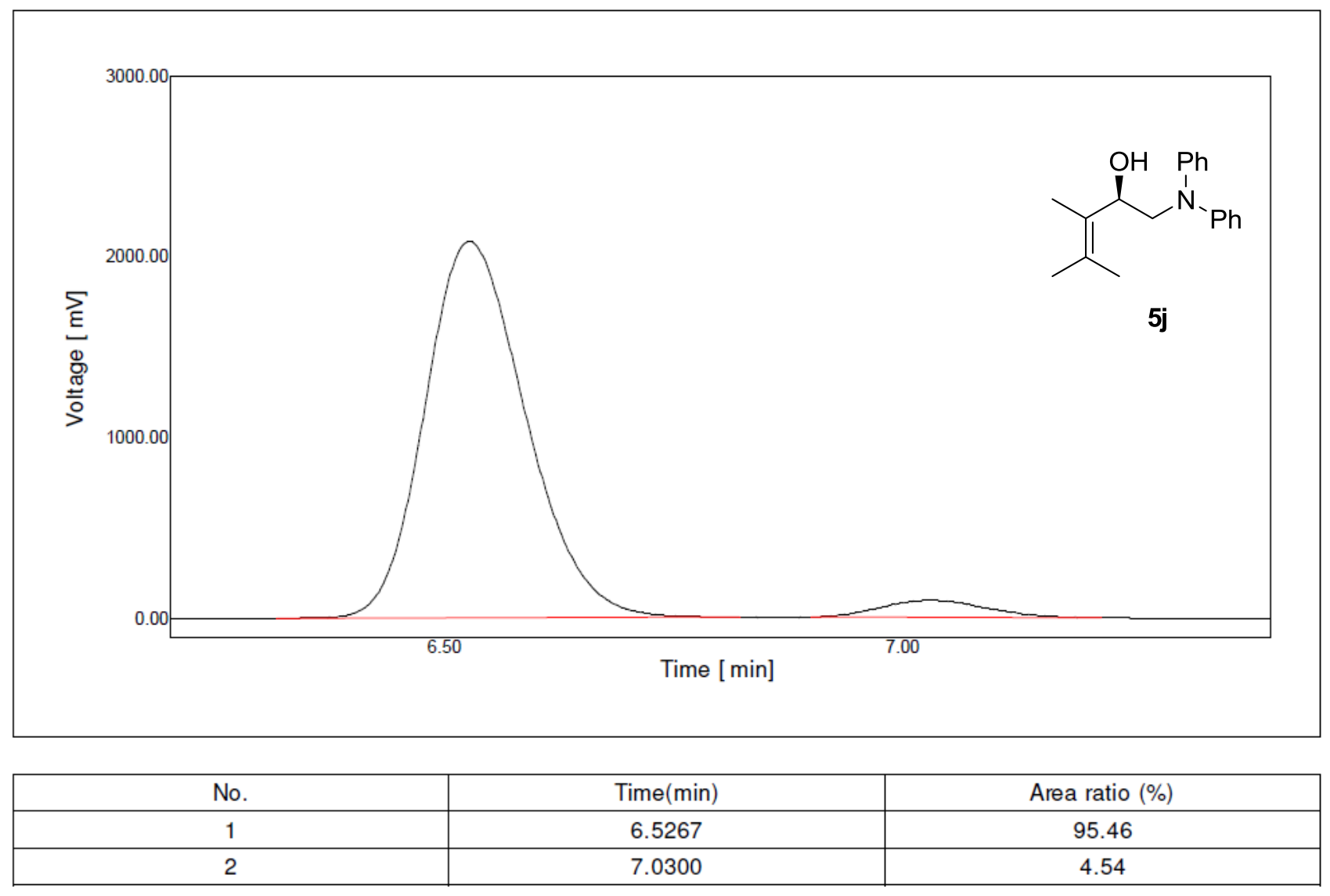




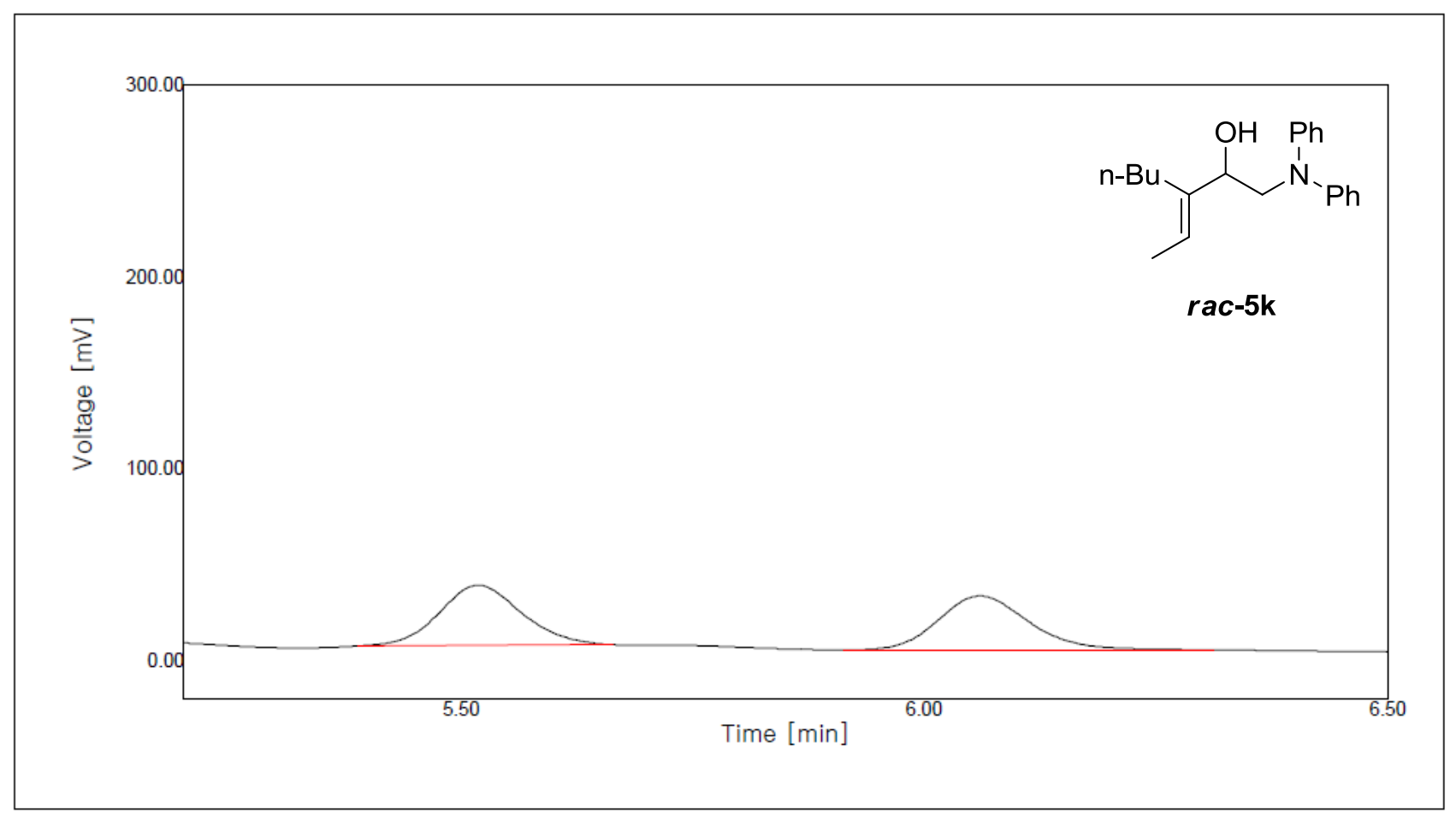

\begin{tabular}{|r|r|r|}
\hline No. & Time $(\mathrm{min})$ & \multicolumn{1}{|c|}{ Area ratio (\%) } \\
\hline 1 & & 5.5183 \\
\hline 2 & 6.0600 & 49.50 \\
\hline
\end{tabular}

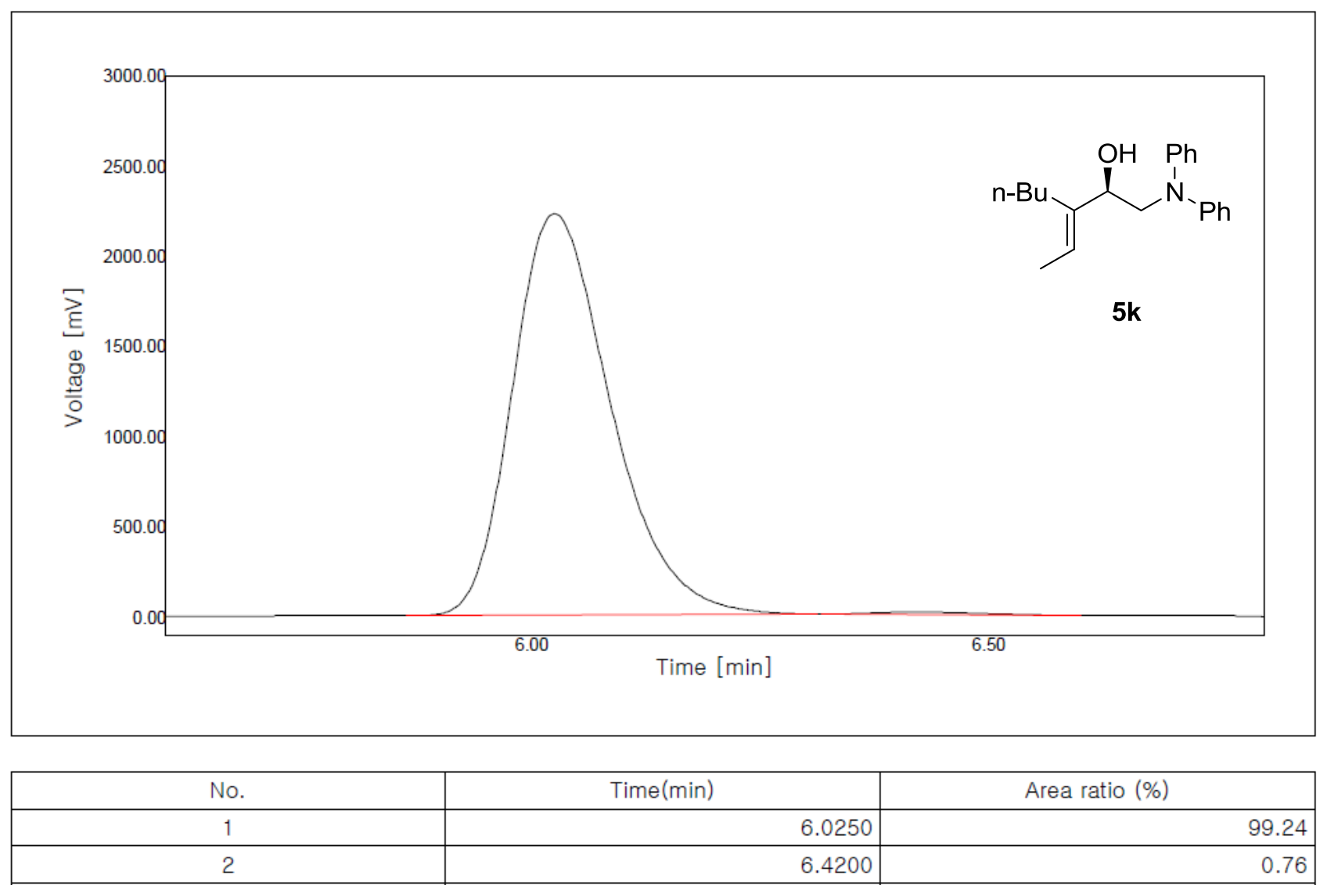




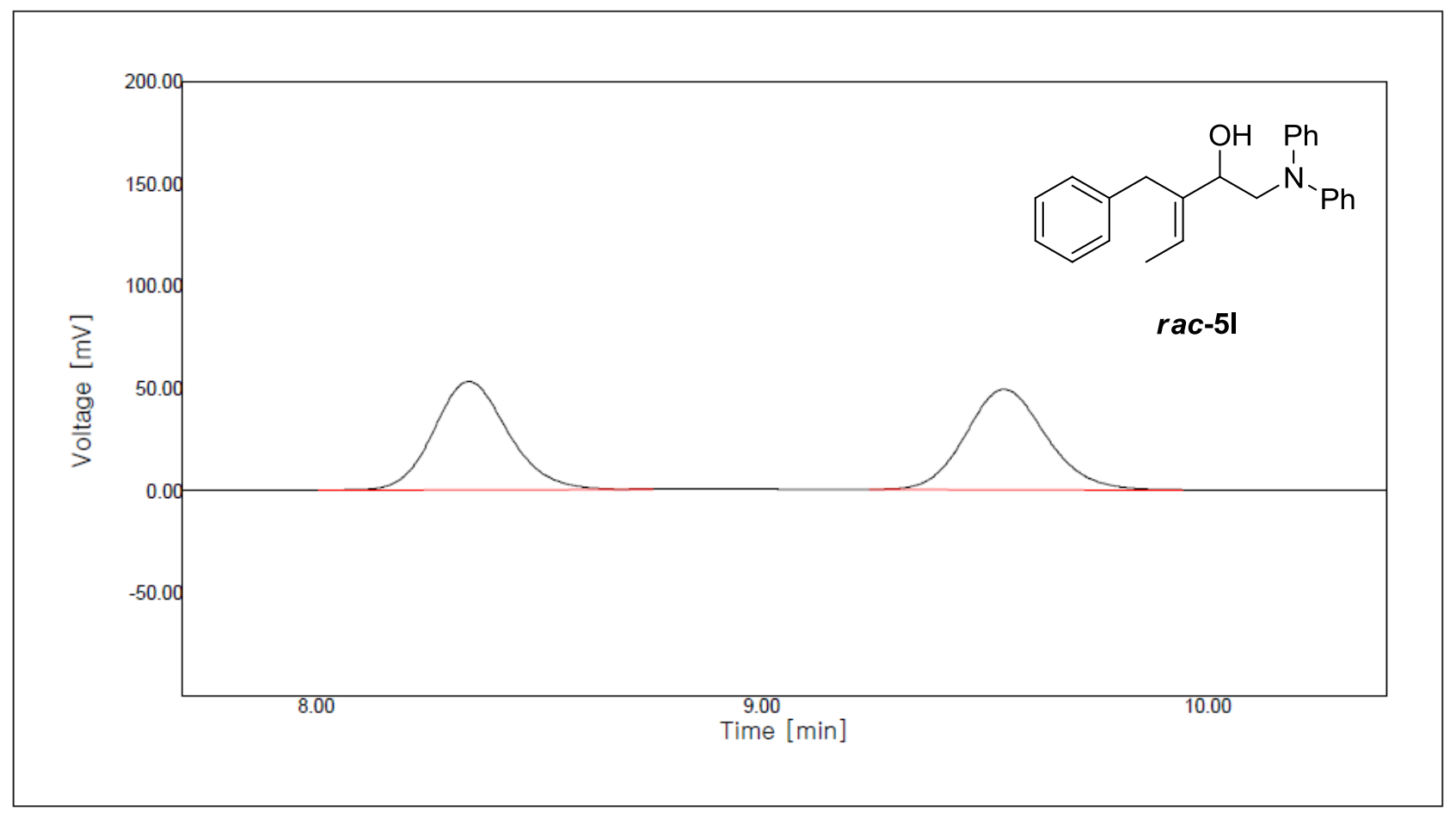

\begin{tabular}{|r|r|r|}
\hline No. & Time $(\mathrm{min})$ & \multicolumn{1}{|c|}{ Area ratio (\%) } \\
\hline 1 & & 8.3433 \\
\hline 2 & 9.5433 & 49.06 \\
\hline
\end{tabular}

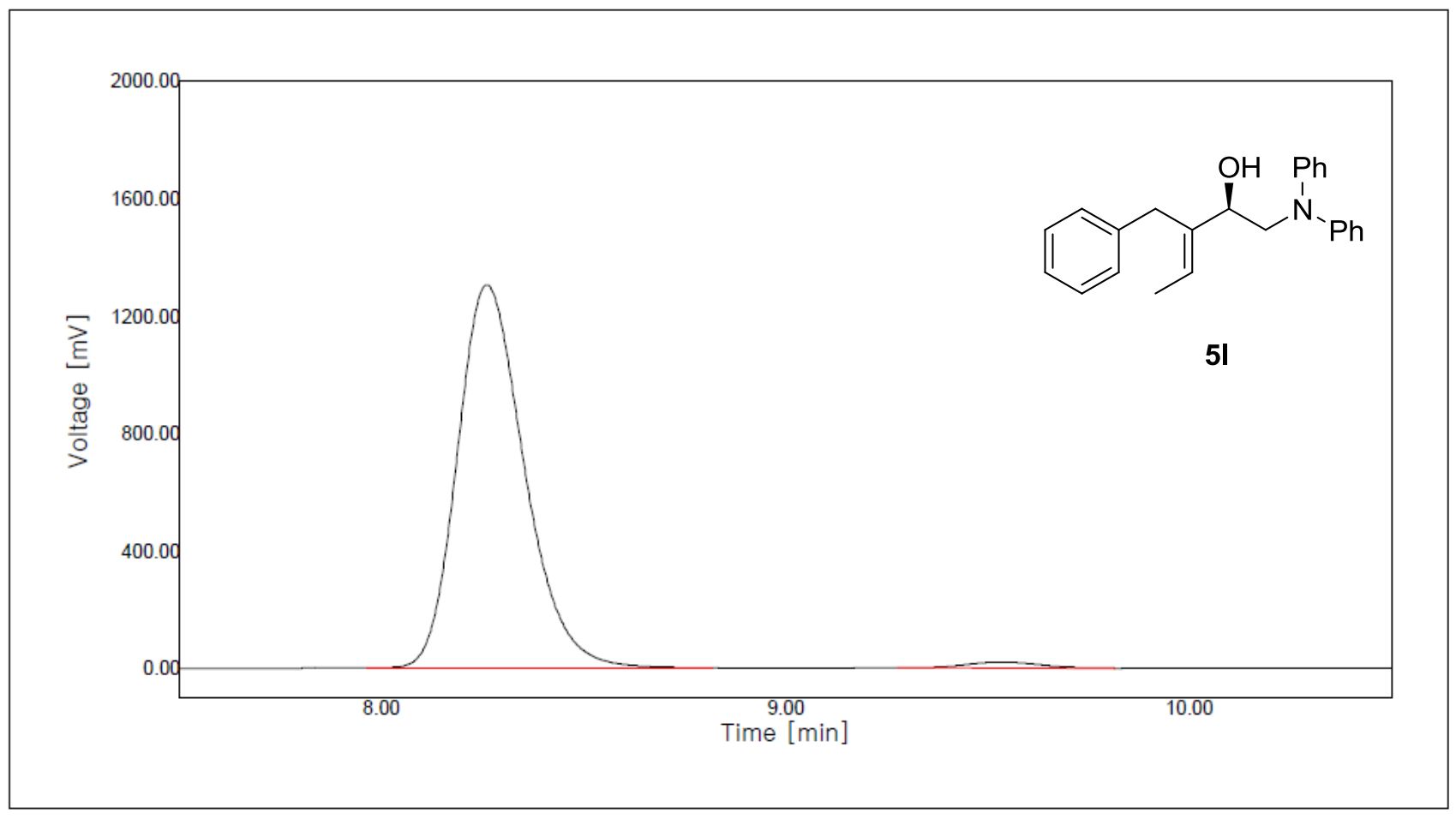

\begin{tabular}{|r|r|r|}
\hline No. & Time $(\mathrm{min})$ & \multicolumn{1}{|c|}{ Area ratio (\%) } \\
\hline 1 & & 8.2600 \\
\hline 2 & 9.5350 & 1.71 \\
\hline
\end{tabular}




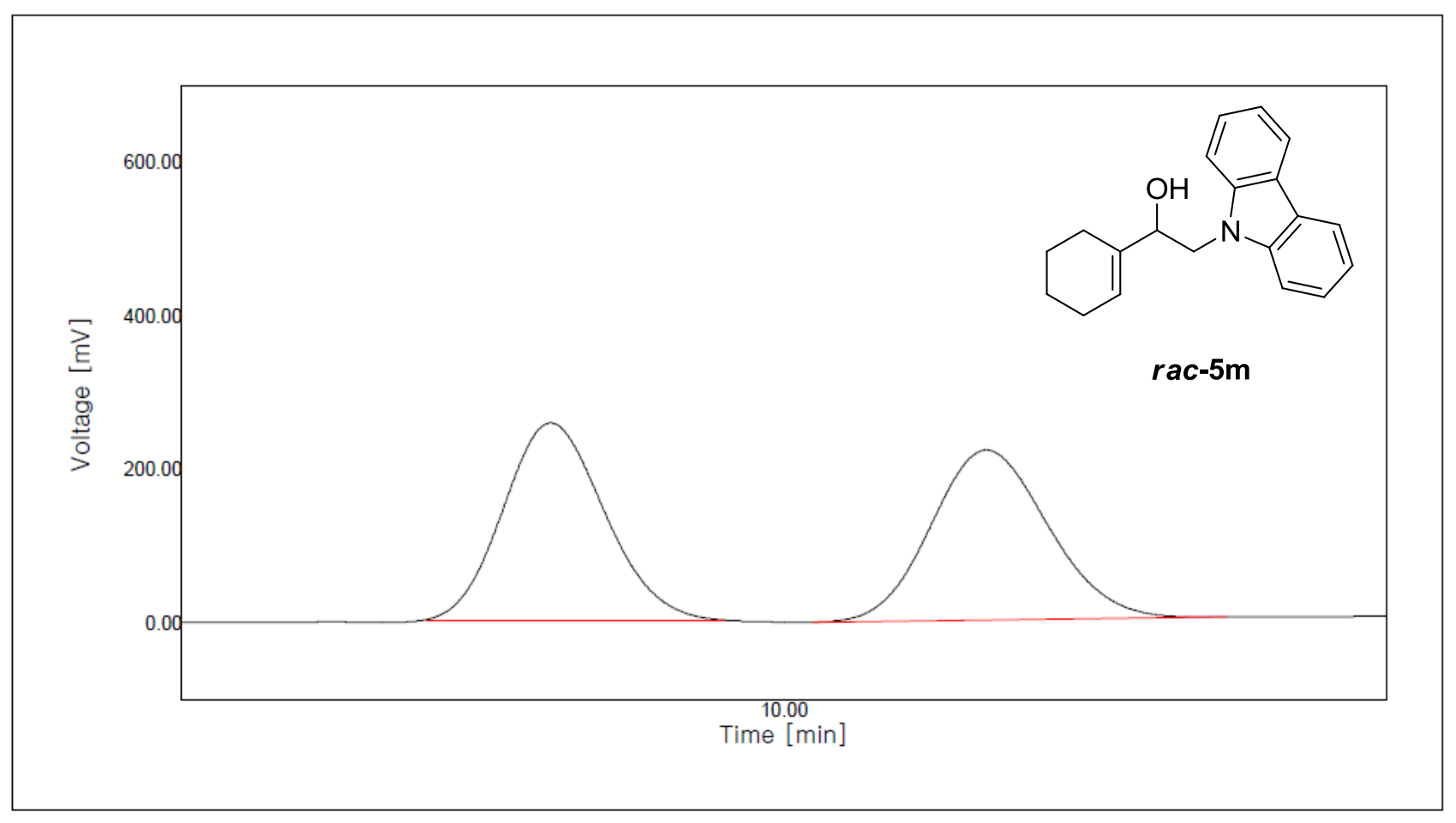

\begin{tabular}{|r|r|r|}
\hline No. & Time $(\mathrm{min})$ & \multicolumn{1}{|c|}{ Area ratio (\%) } \\
\hline 1 & & 9.6133 \\
\hline 2 & 10.3367 & 49.06 \\
\hline
\end{tabular}

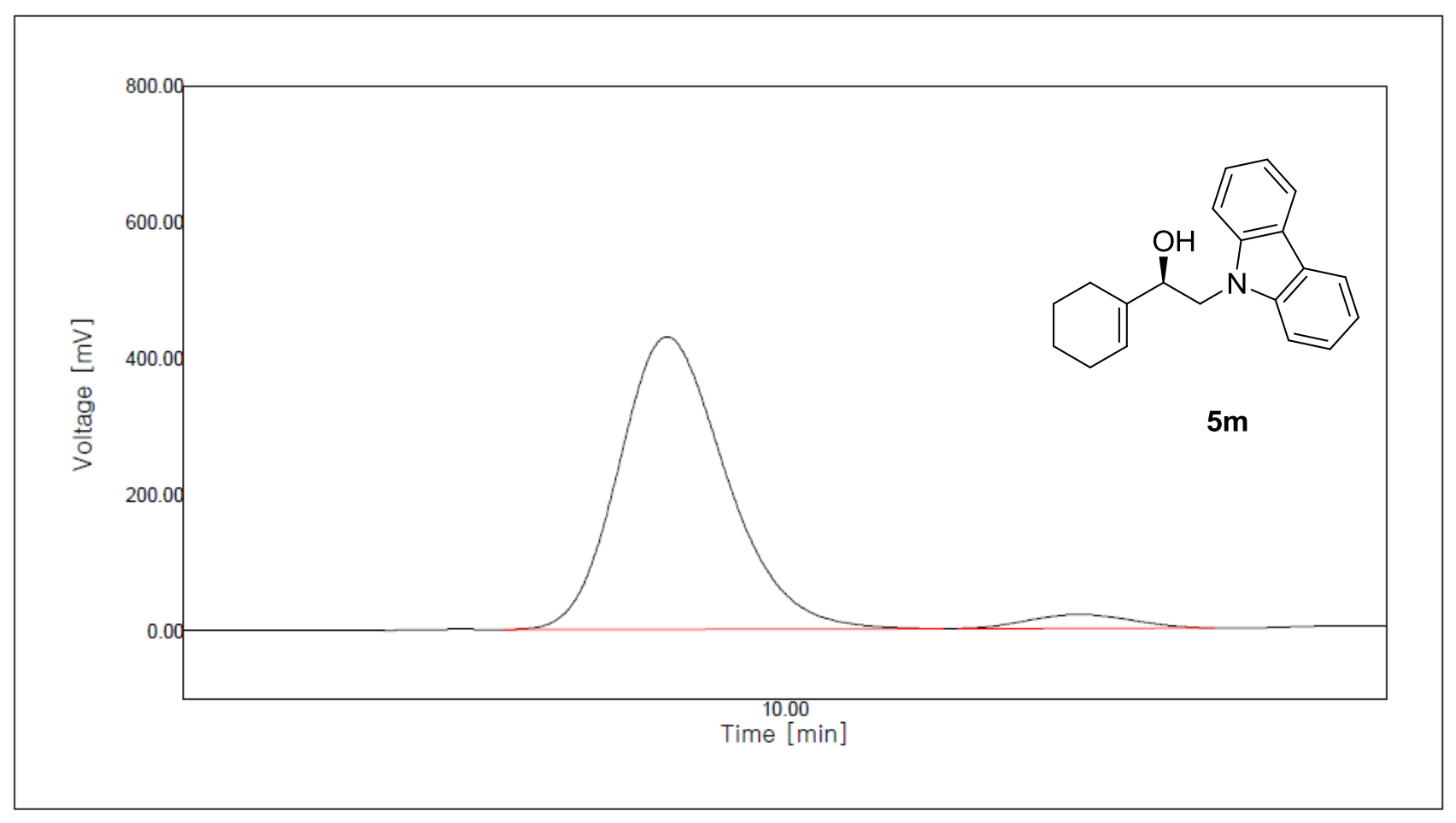

\begin{tabular}{|r|r|r|}
\hline No. & Time $(\mathrm{min})$ & \multicolumn{1}{|c|}{ Area ratio (\%) } \\
\hline 1 & & 9.8033 \\
\hline 2 & 10.4900 & 4.42 \\
\hline
\end{tabular}




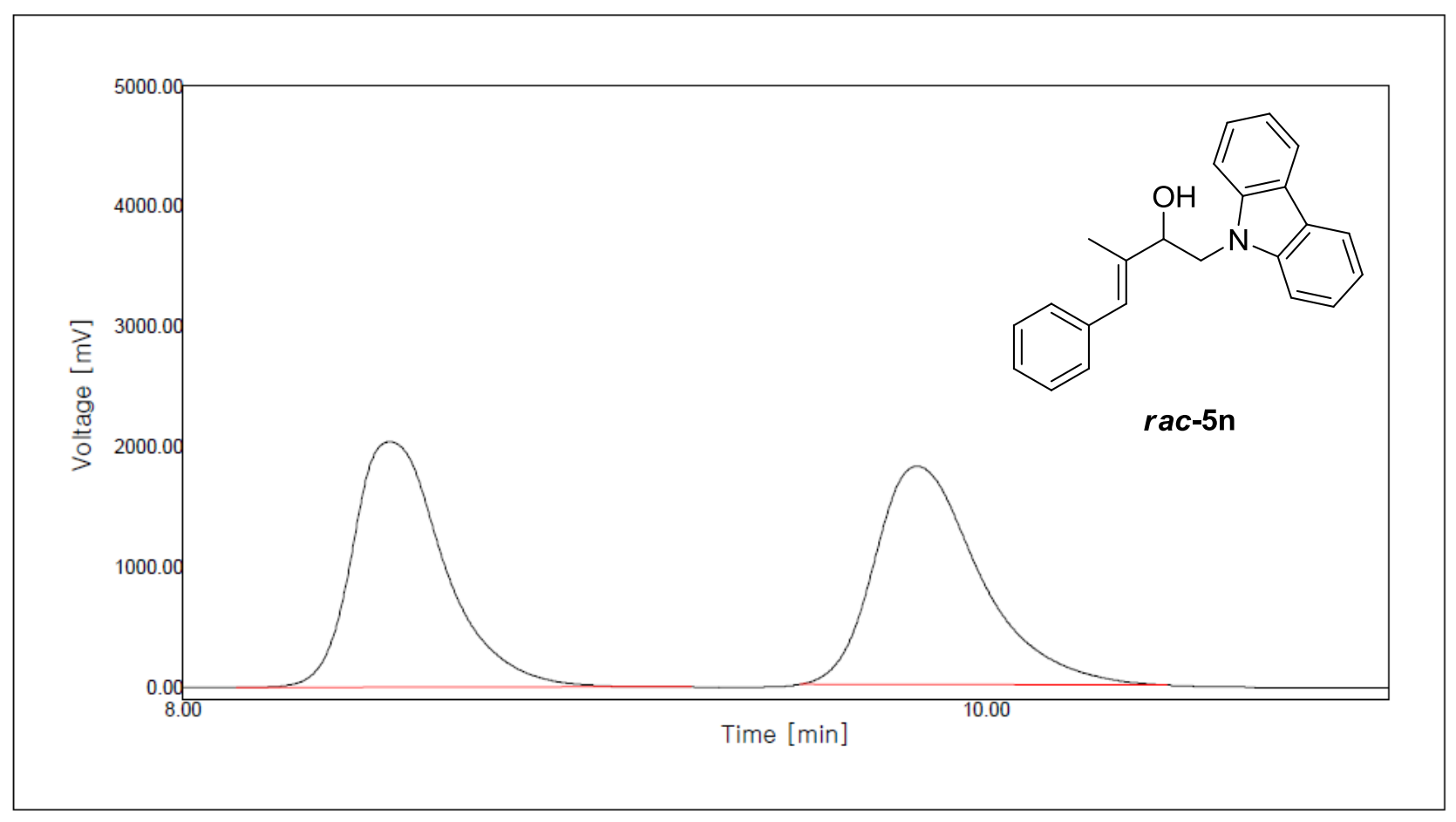

\begin{tabular}{|r|r|r|}
\hline No. & Time $(\mathrm{min})$ & \multicolumn{1}{|c|}{ Area ratio (\%) } \\
\hline 1 & & 8.5150 \\
\hline 2 & 9.8283 & 59.34 \\
\hline
\end{tabular}

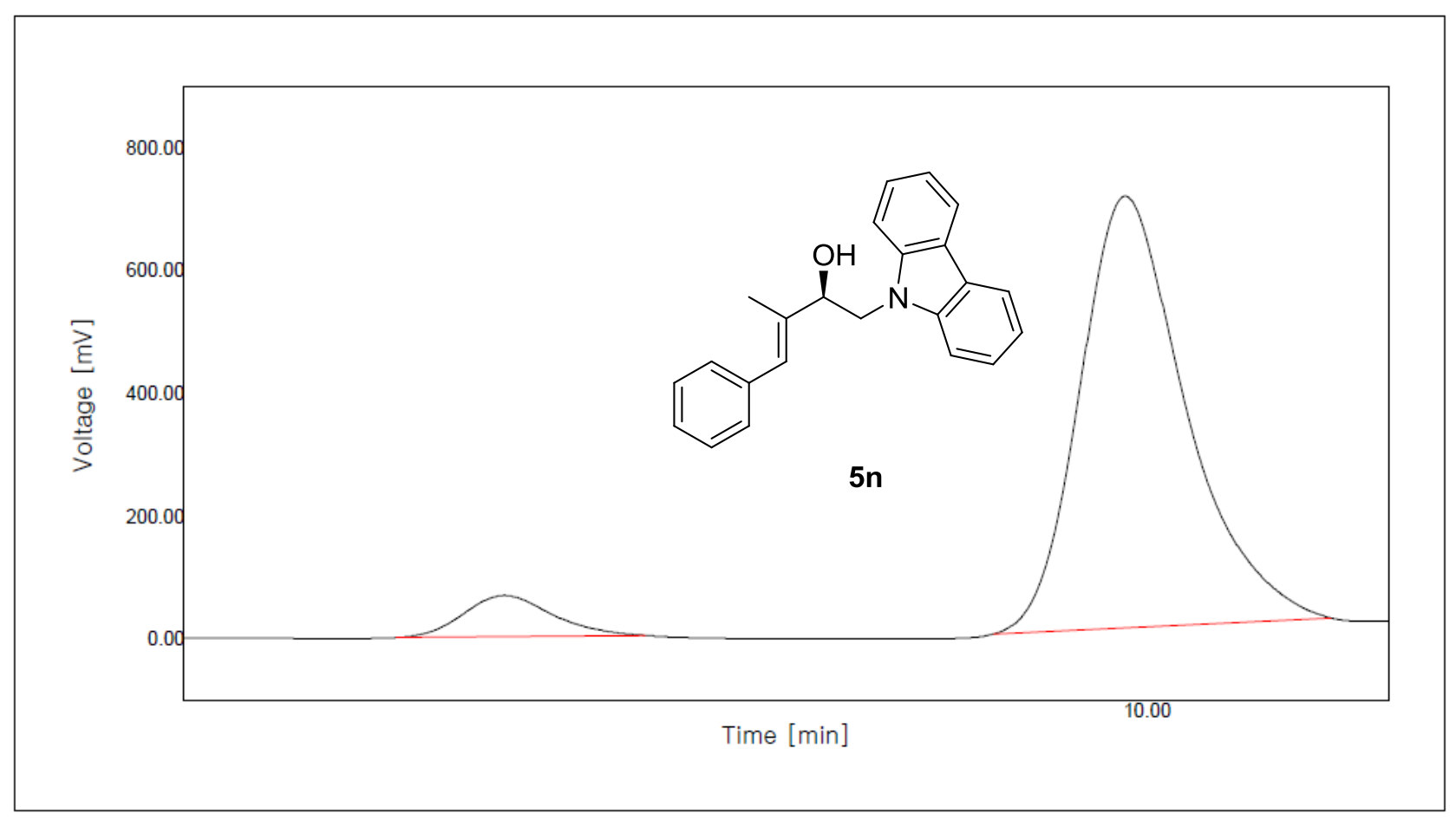

\begin{tabular}{|r|r|r|}
\hline No. & Time $(\mathrm{min})$ & \multicolumn{1}{|c|}{ Area ratio (\%) } \\
\hline 1 & & 8.6650 \\
\hline 2 & 9.9550 & 9.40 \\
\hline
\end{tabular}




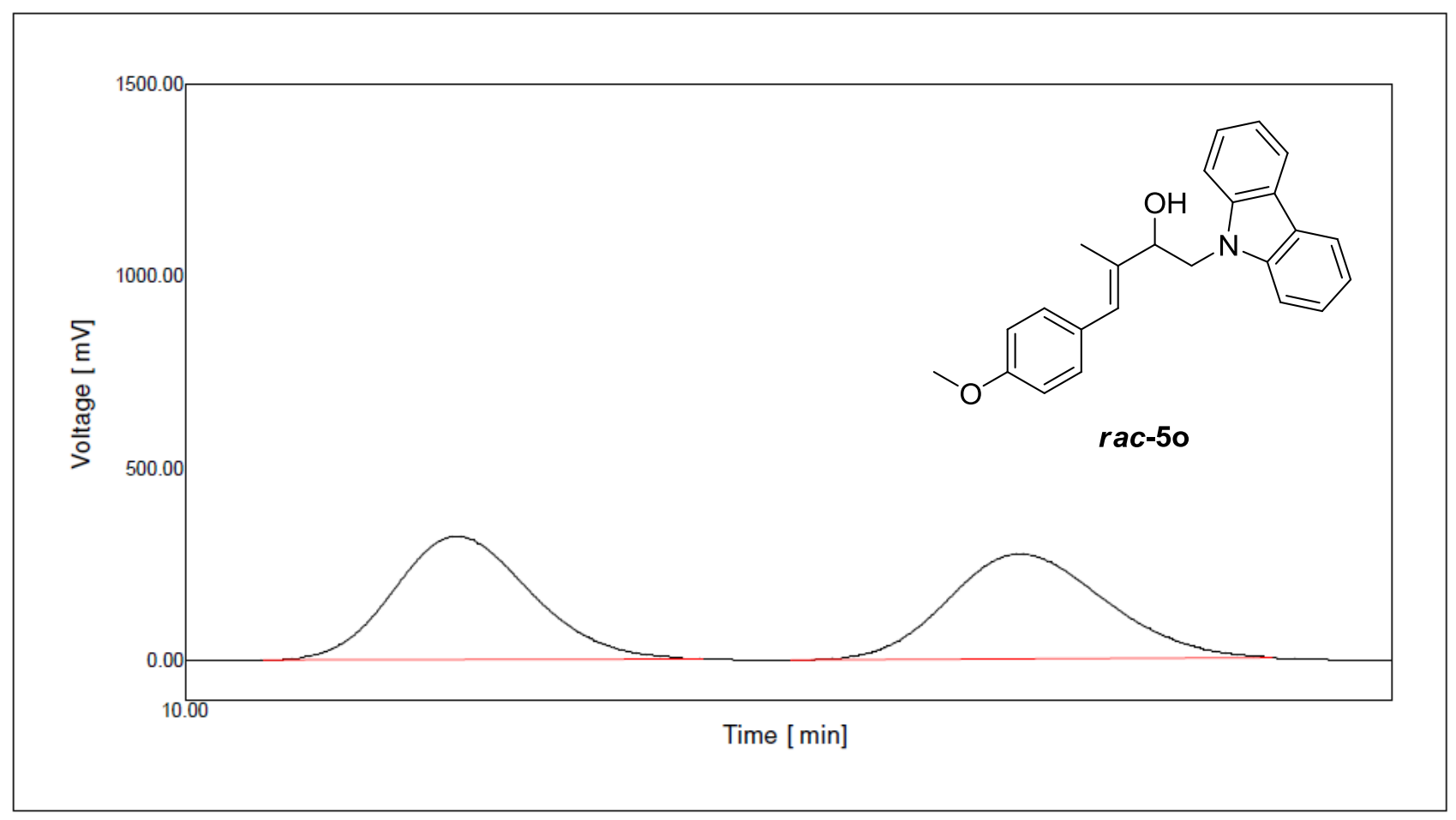

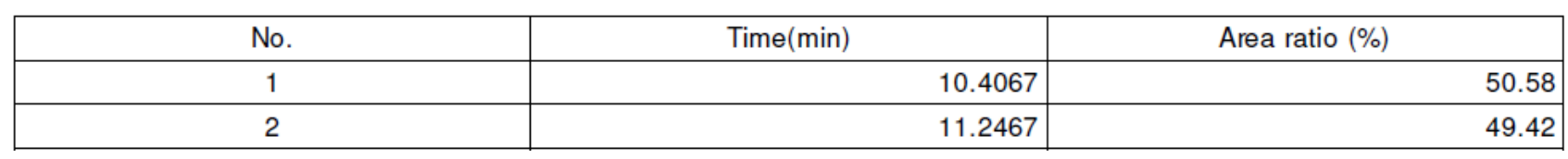

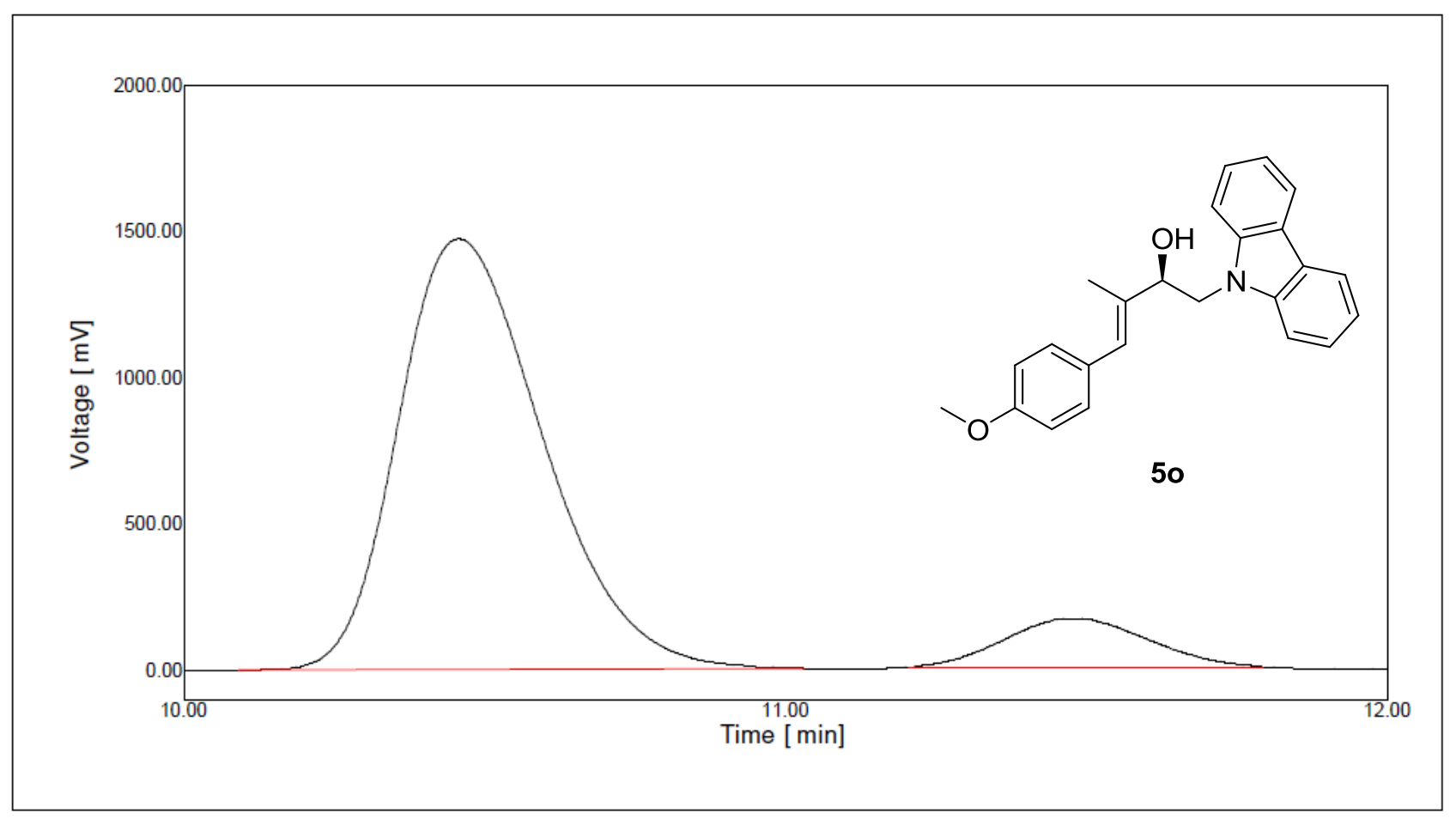

\begin{tabular}{|c|c|c|}
\hline No. & Time $(\mathrm{min})$ & Area ratio (\%) \\
\hline 1 & 10.4583 & 90.08 \\
\hline 2 & 11.4783 & 9.92 \\
\hline
\end{tabular}




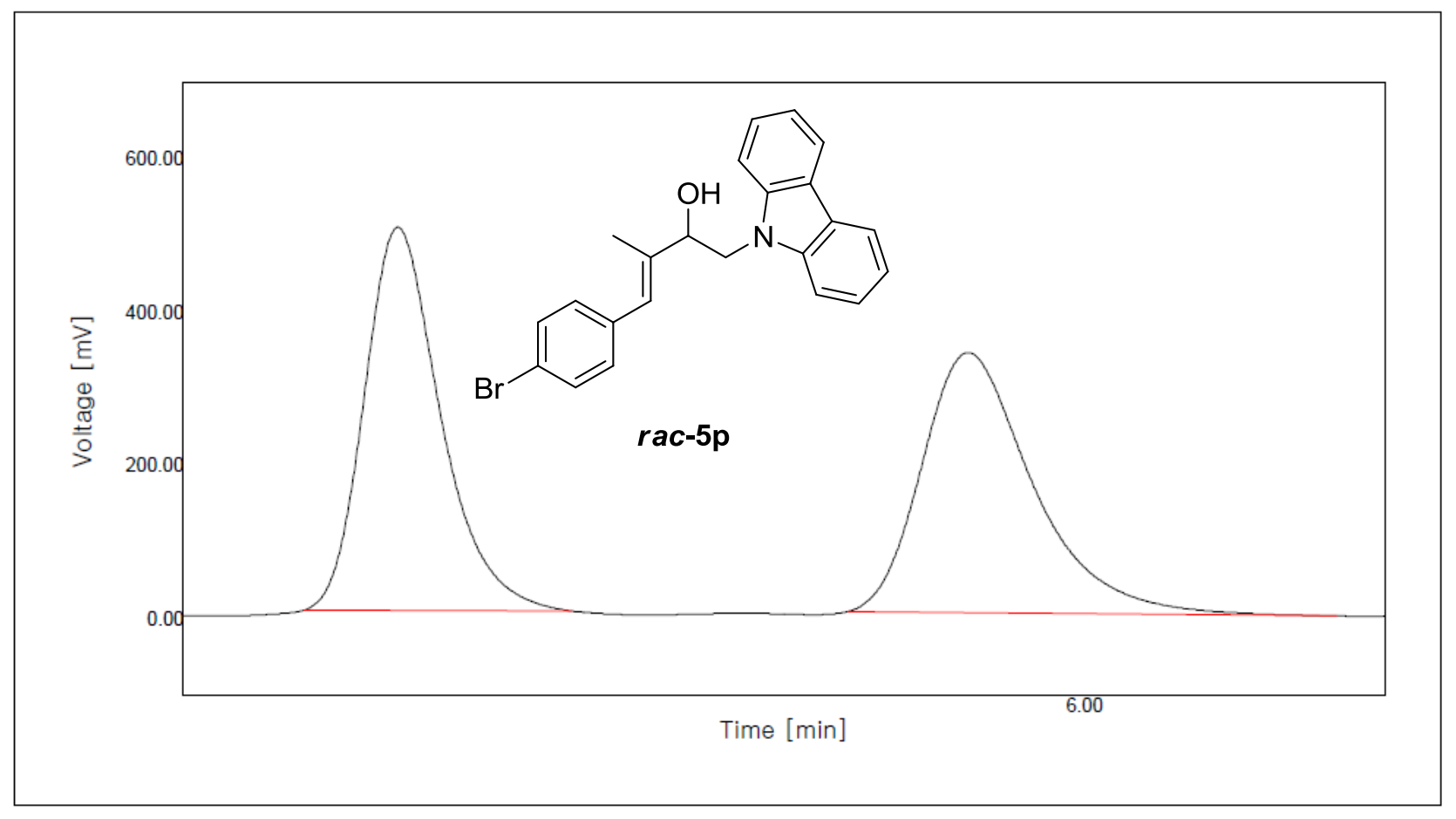

\begin{tabular}{|c|c|c|}
\hline No. & Time(min) & Area ratio (\%) \\
\hline 1 & 4.8567 & 50.28 \\
\hline 2 & 5.8067 & 49.72 \\
\hline
\end{tabular}

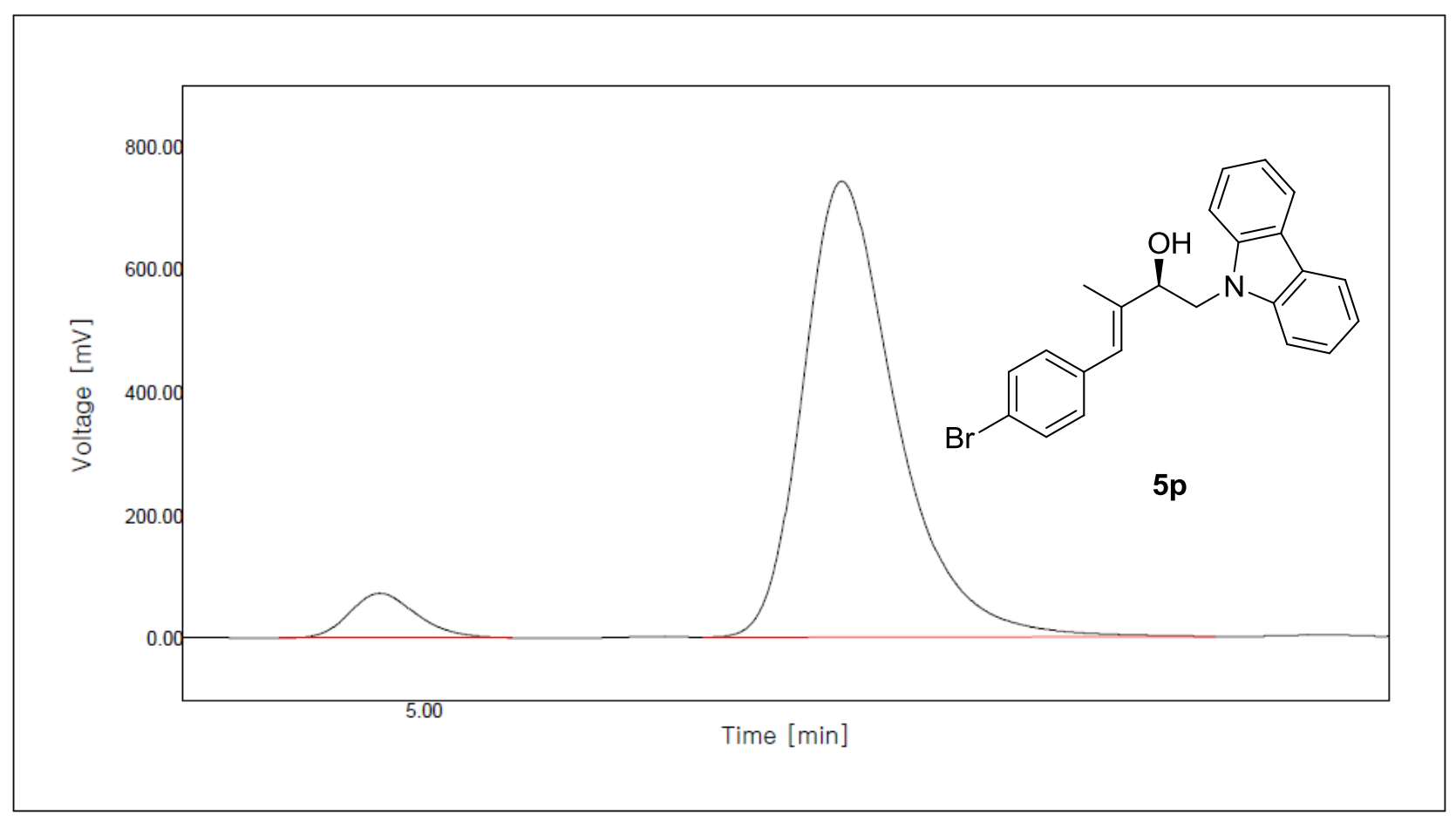

\begin{tabular}{|c|c|c|}
\hline No. & Time(min) & Area ratio (\%) \\
\hline 1 & 4.9083 & 6.62 \\
\hline 2 & 5.8667 & 93.38 \\
\hline
\end{tabular}




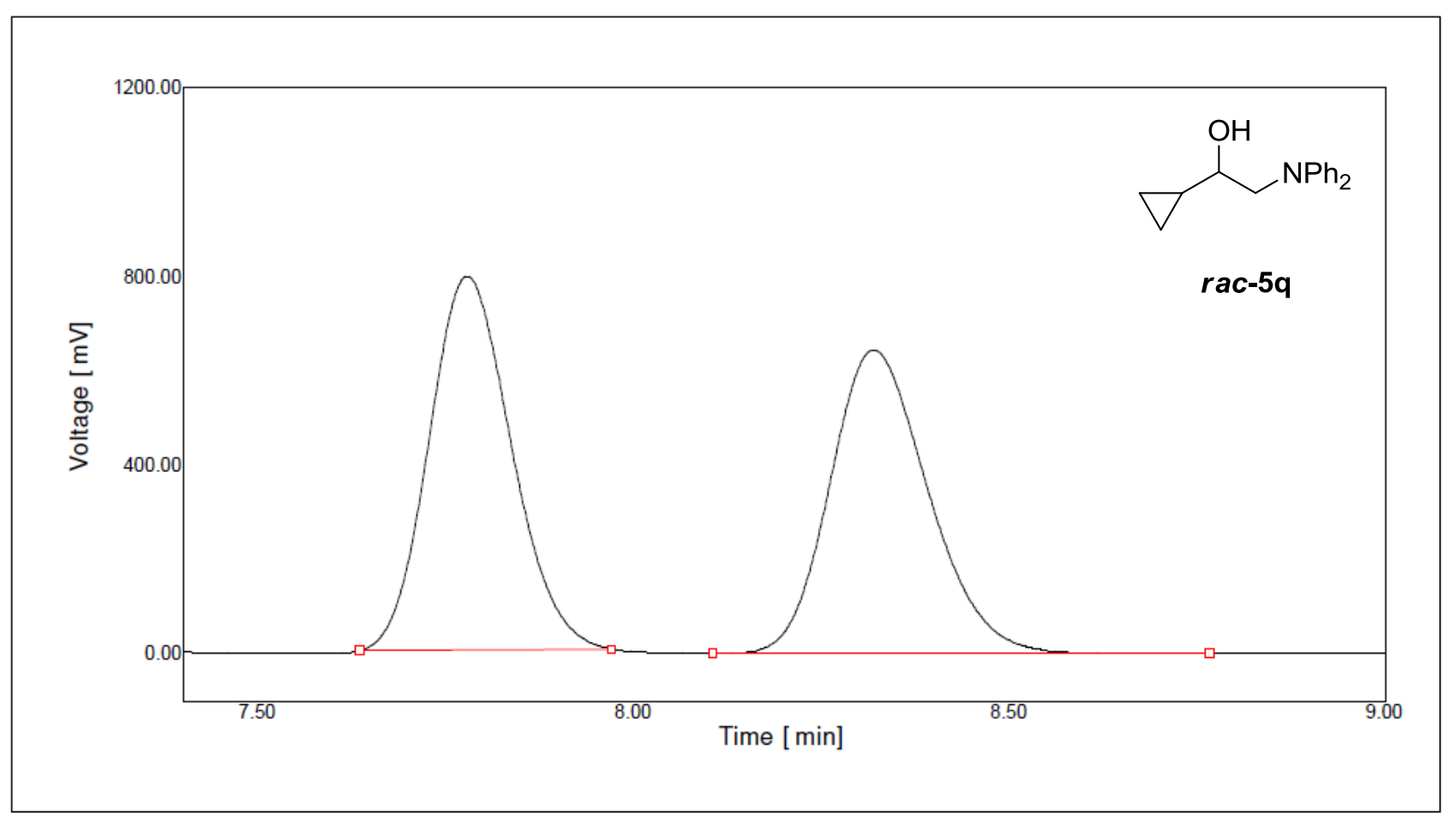

\begin{tabular}{|c|c|c|}
\hline No. & Time $(\mathrm{min})$ & Area ratio (\%) \\
\hline 1 & 7.7783 & 50.84 \\
\hline 2 & 8.3200 & 49.16 \\
\hline
\end{tabular}

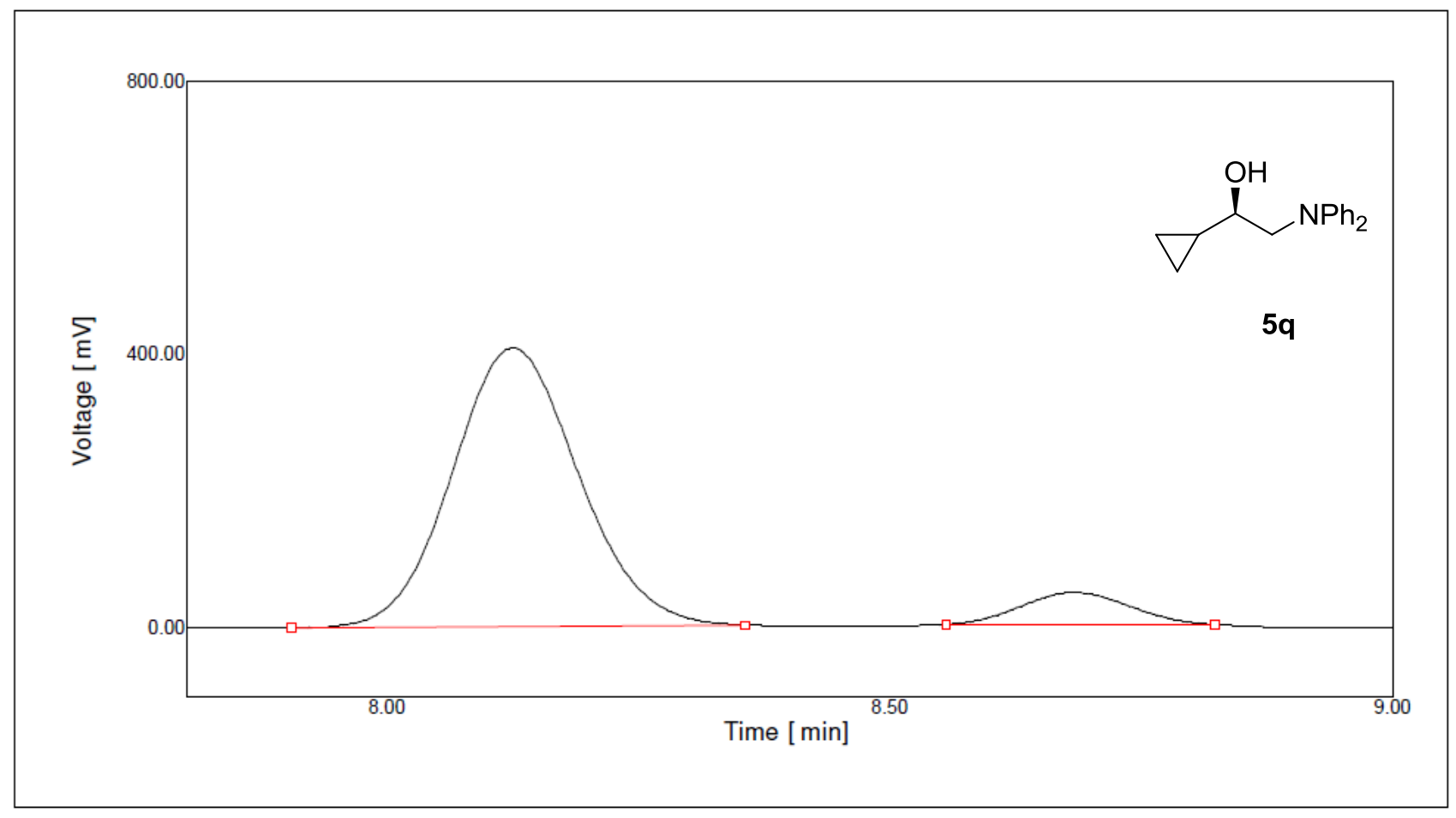

\begin{tabular}{|c|c|c|}
\hline No. & Time $(\mathrm{min})$ & Area ratio (\%) \\
\hline 1 & 8.1250 & 90.94 \\
\hline 2 & 8.6833 & 9.06 \\
\hline
\end{tabular}




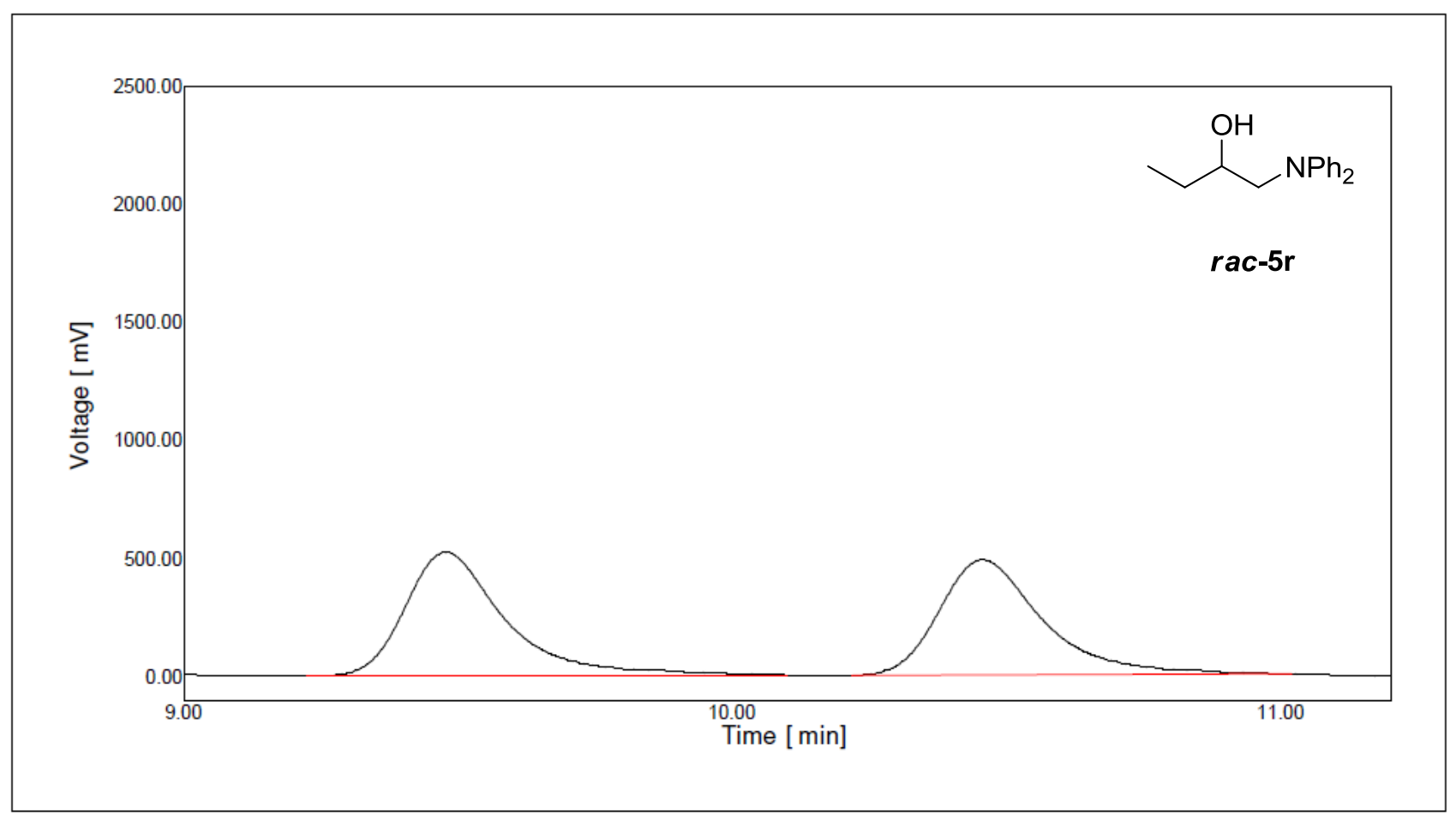

\begin{tabular}{|c|c|c|}
\hline Peak \# & Time (min) & Area (\%) \\
\hline 1 & 9.4783 & 50.61 \\
\hline 2 & 10.4567 & 49.39 \\
\hline
\end{tabular}

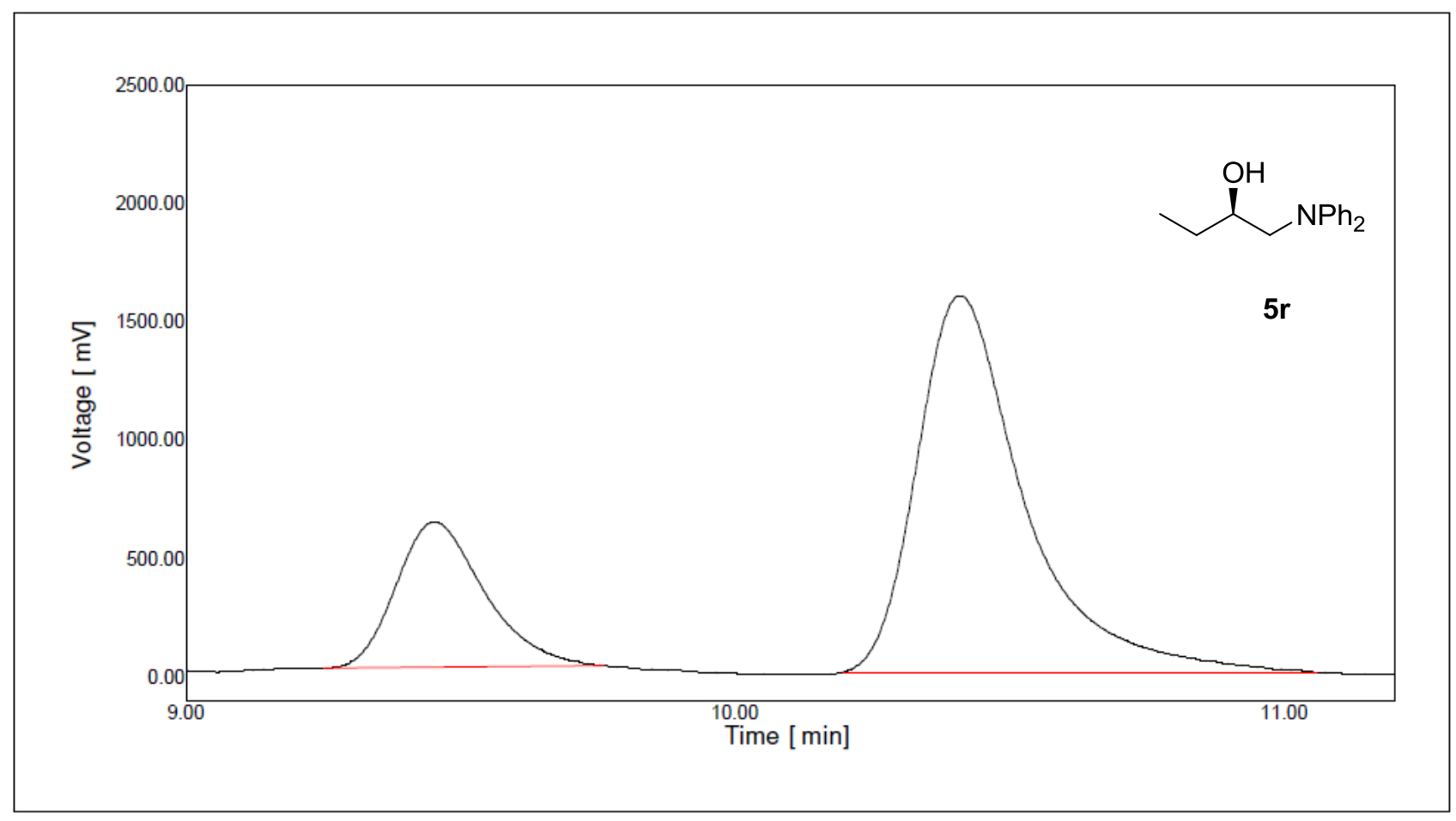

\begin{tabular}{|c|r|r|}
\hline Peak \# & Time $(\mathrm{min})$ & \multicolumn{1}{|c|}{ Area (\%) } \\
\hline 1 & 9.4533 & 24.00 \\
\hline 2 & 10.4100 & 76.00 \\
\hline
\end{tabular}




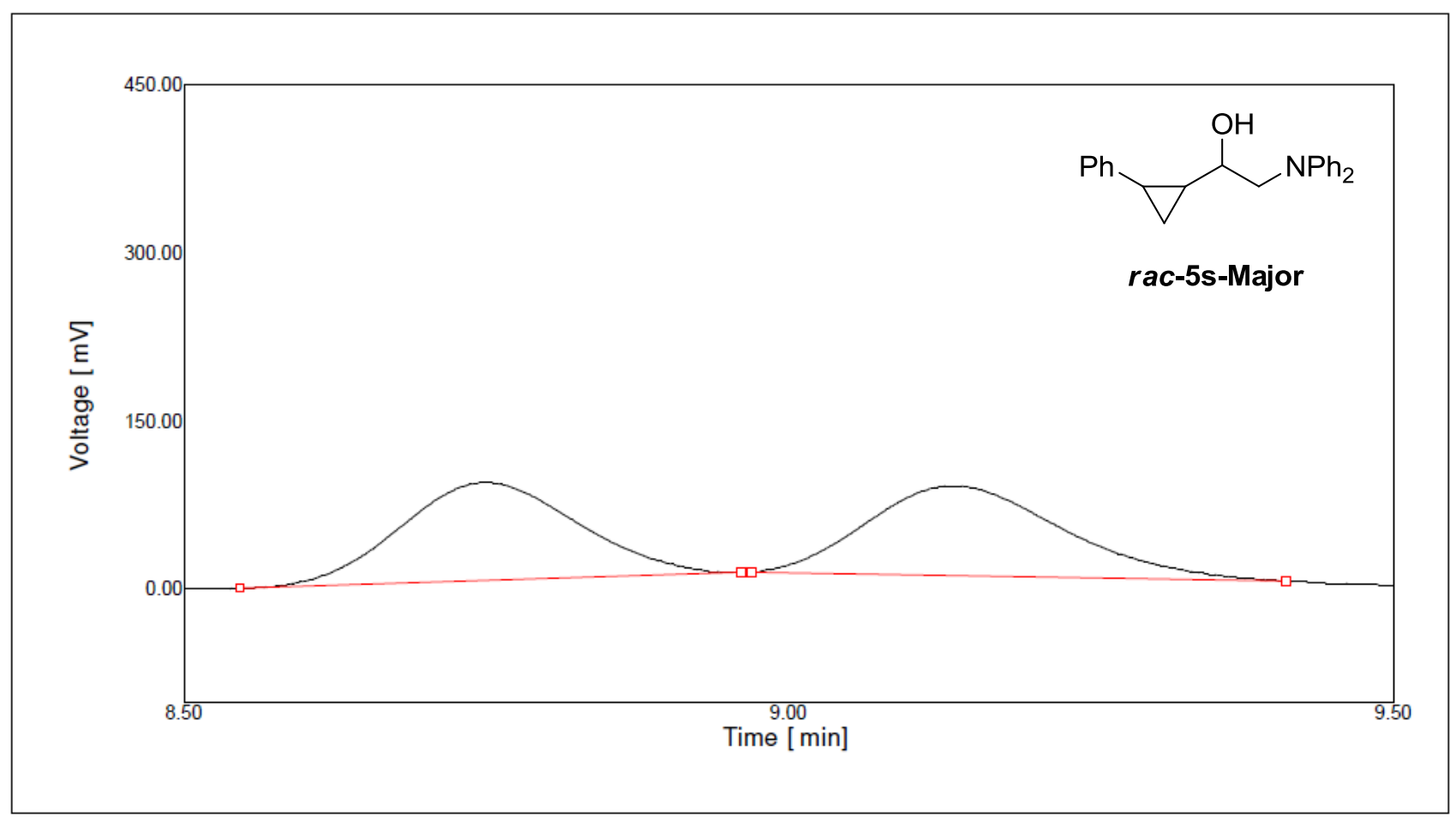

\begin{tabular}{|c|c|c|}
\hline No. & Time $(\mathrm{min})$ & Area ratio (\%) \\
\hline 1 & 8.7500 & 49.88 \\
\hline 2 & 9.1367 & 50.12 \\
\hline
\end{tabular}

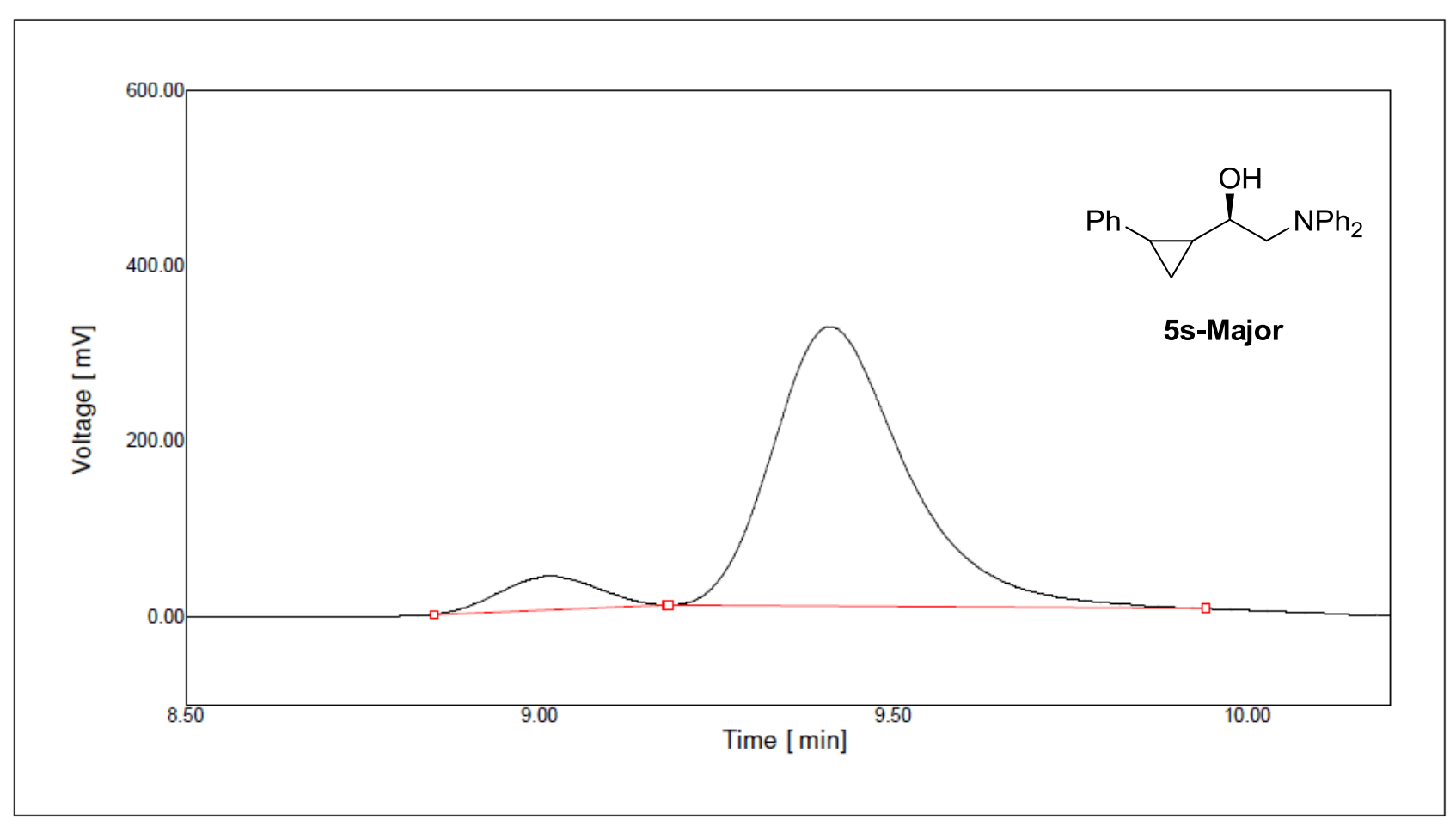

\begin{tabular}{|c|c|c|}
\hline No. & Time $(\min )$ & Area ratio $(\%)$ \\
\hline 1 & 9.0150 & 8.12 \\
\hline 2 & 9.4100 & 91.88 \\
\hline
\end{tabular}




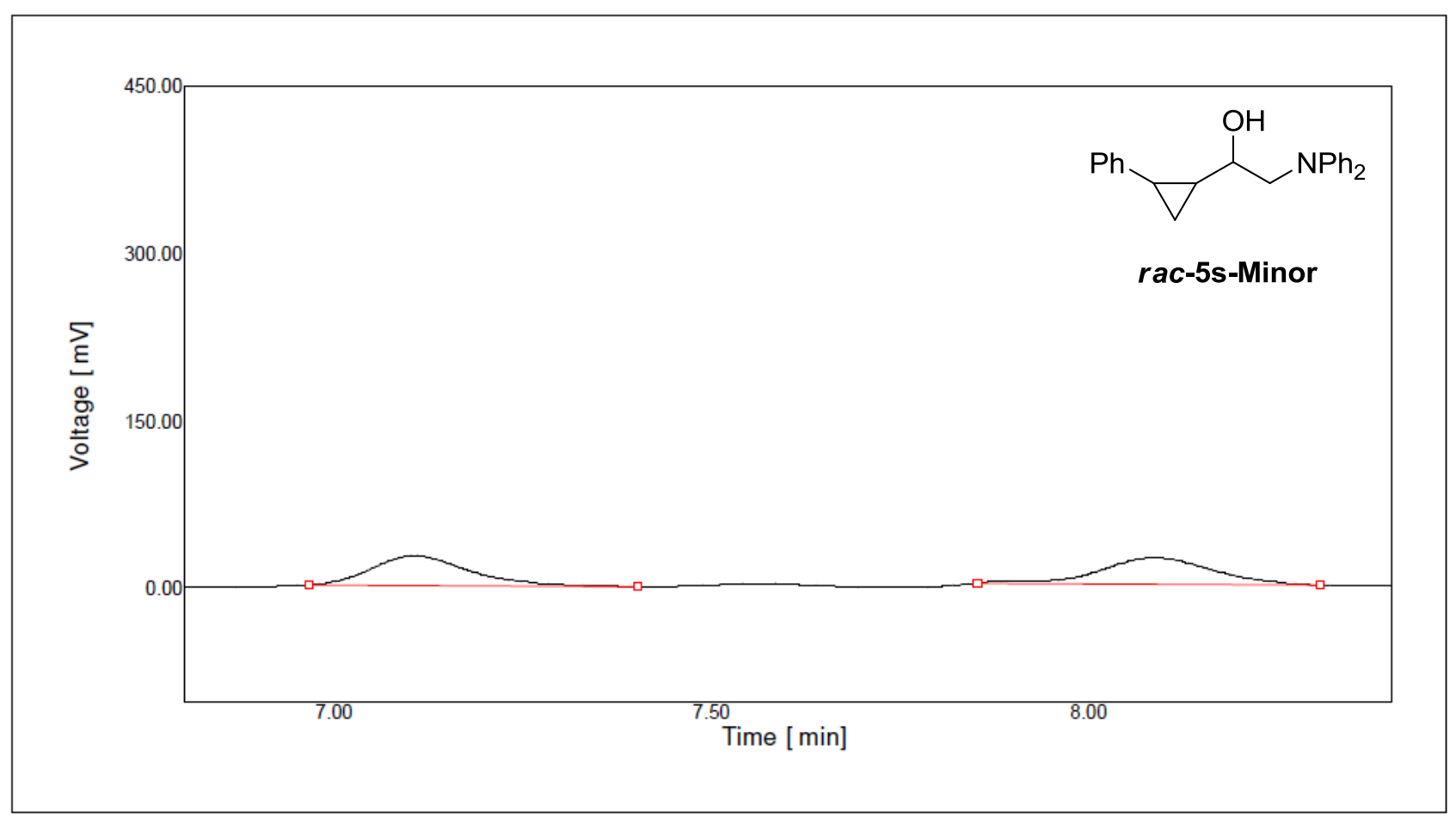

\begin{tabular}{|c|c|c|}
\hline No. & Time $(\mathrm{min})$ & Area ratio (\%) \\
\hline 1 & 7.1067 & 49.15 \\
\hline 2 & 8.0867 & 50.85 \\
\hline
\end{tabular}

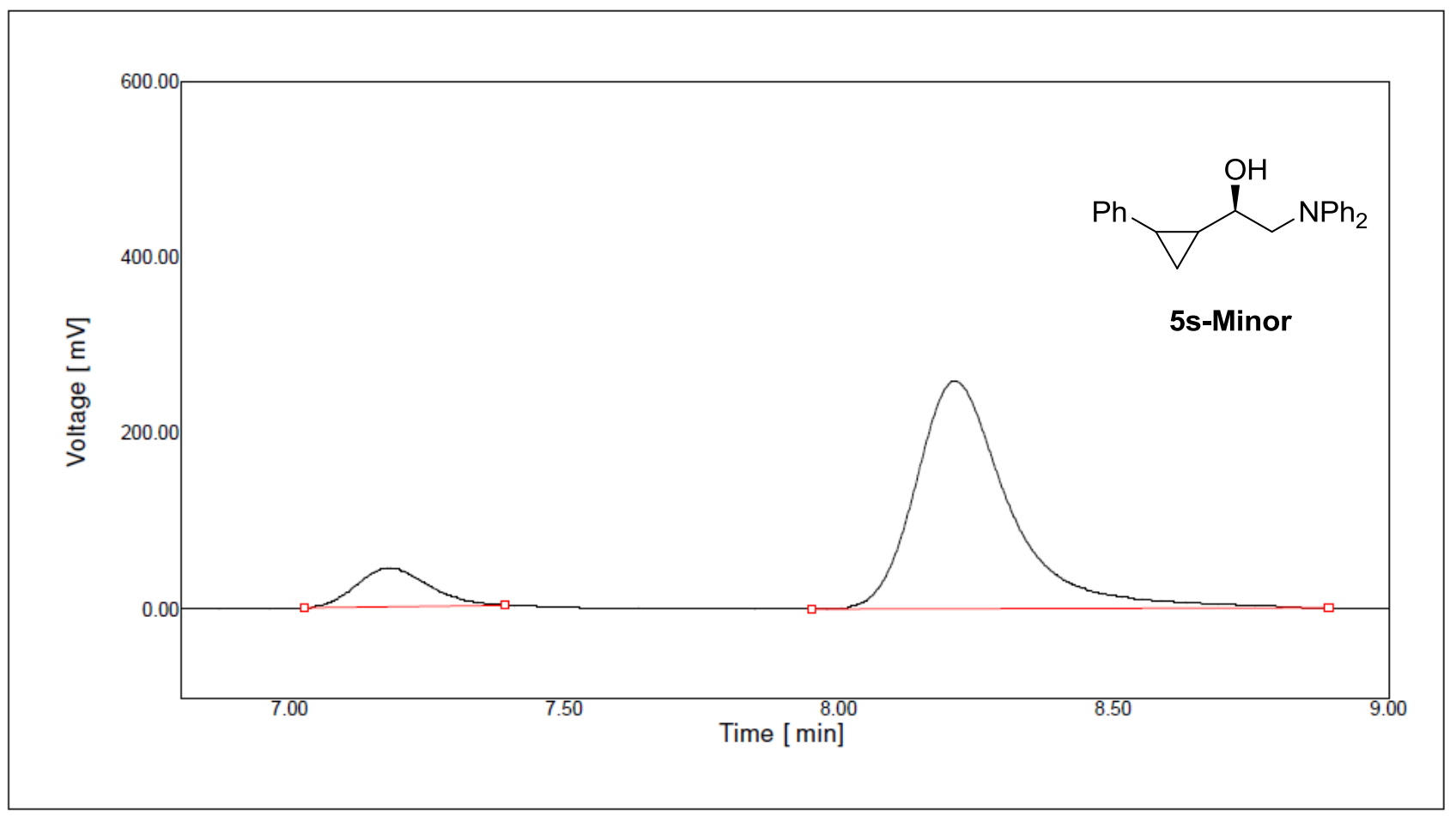

\begin{tabular}{|c|c|c|}
\hline No. & Time $(\mathrm{min})$ & Area ratio (\%) \\
\hline 1 & 7.1817 & 11.60 \\
\hline 2 & 8.2117 & 88.40 \\
\hline
\end{tabular}




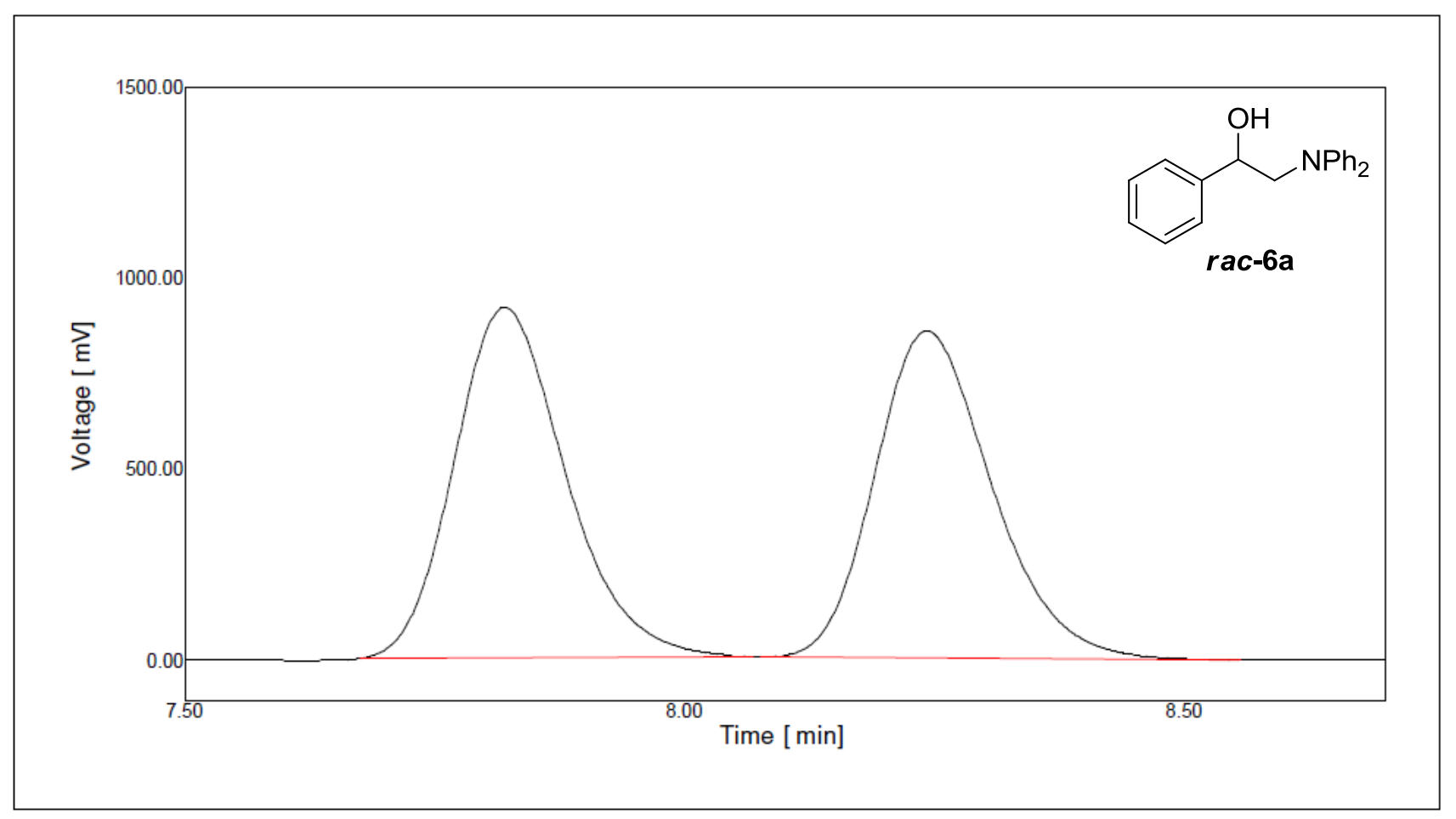

\begin{tabular}{|c|c|c|}
\hline No. & Time $(\mathrm{min})$ & Area ratio (\%) \\
\hline 1 & 7.8200 & 50.53 \\
\hline 2 & 8.2433 & 49.47 \\
\hline
\end{tabular}

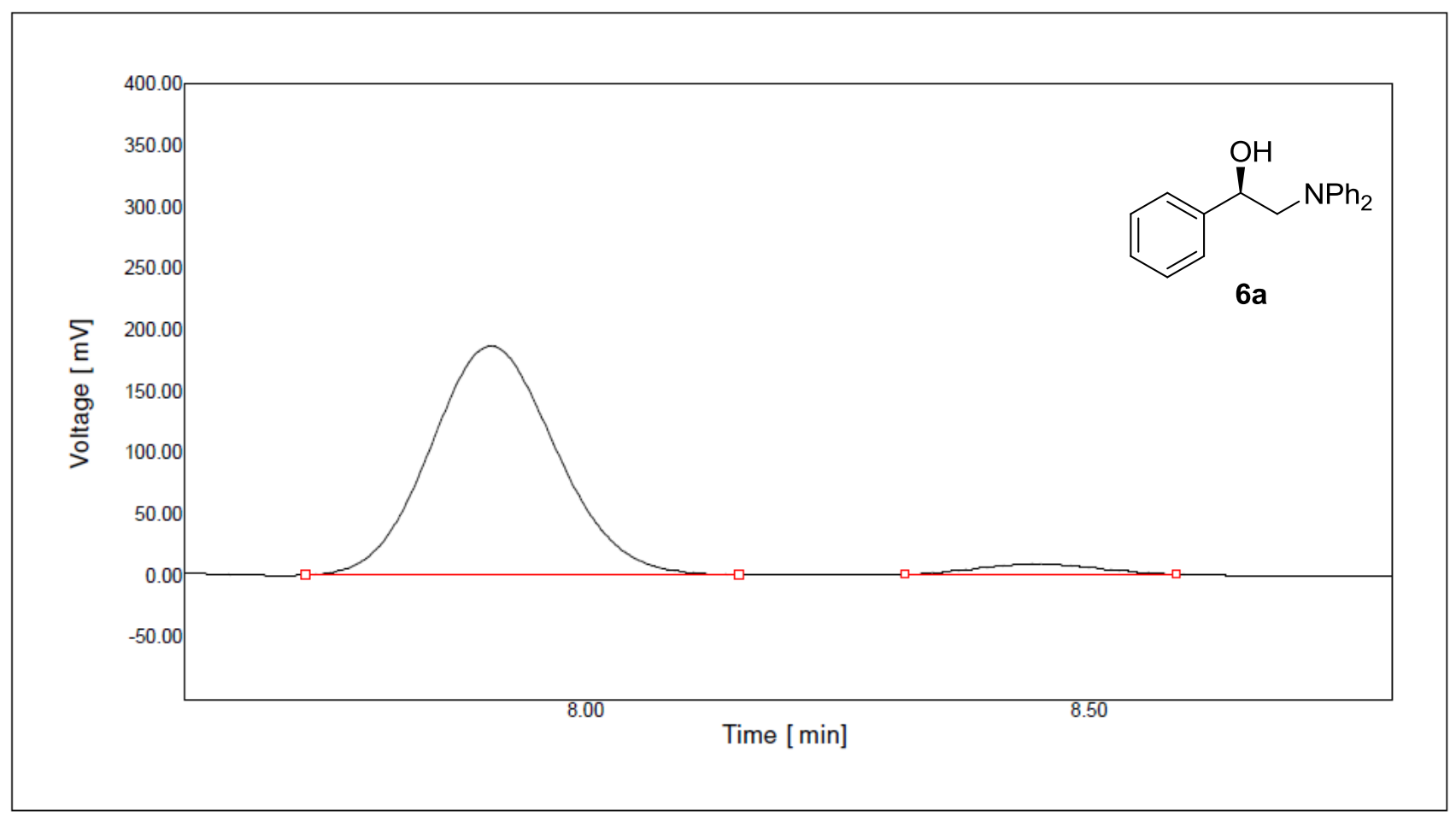

\begin{tabular}{|c|c|c|}
\hline No. & Time $(\mathrm{min})$ & Area ratio $(\%)$ \\
\hline 1 & 7.9050 & 95.91 \\
\hline 2 & 8.4483 & 4.09 \\
\hline
\end{tabular}




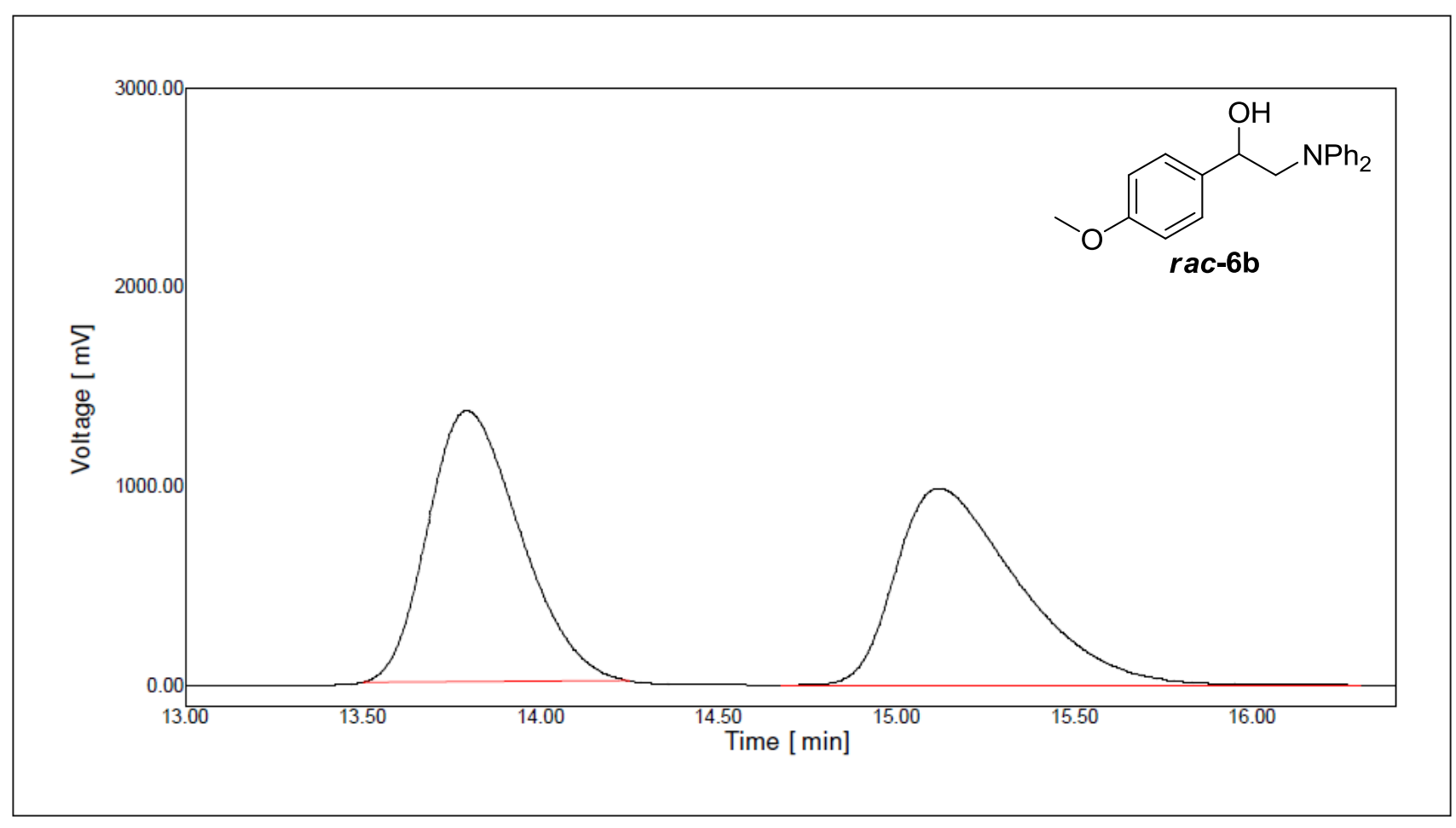

\begin{tabular}{|c|c|c|}
\hline No. & Time $(\mathrm{min})$ & Area ratio (\%) \\
\hline 1 & 13.7933 & 50.85 \\
\hline 2 & 15.1183 & 49.15 \\
\hline
\end{tabular}

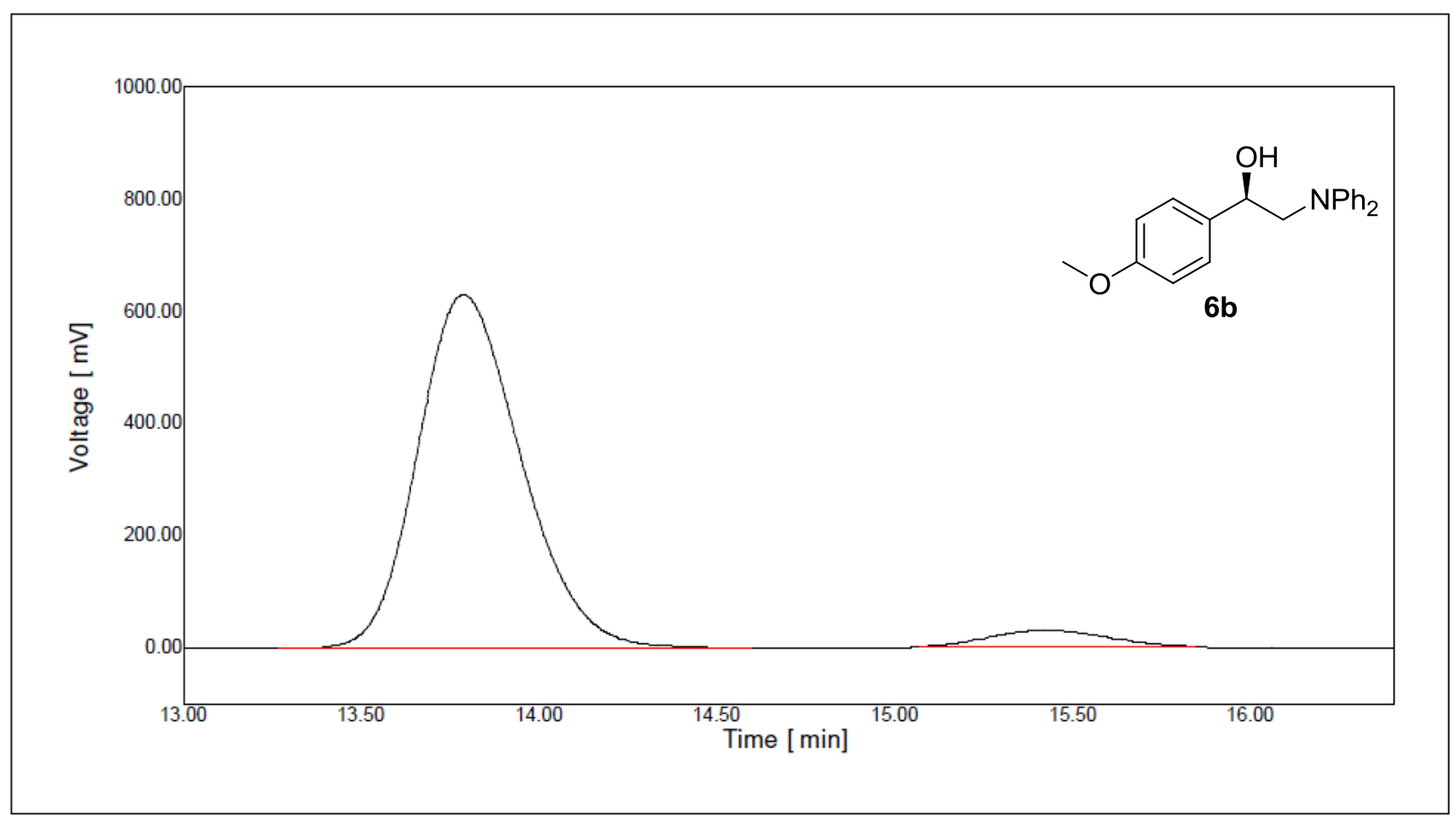

\begin{tabular}{|c|c|c|}
\hline No. & Time $(\mathrm{min})$ & Area ratio (\%) \\
\hline 1 & 13.7883 & 95.04 \\
\hline 2 & 15.4233 & 4.96 \\
\hline
\end{tabular}




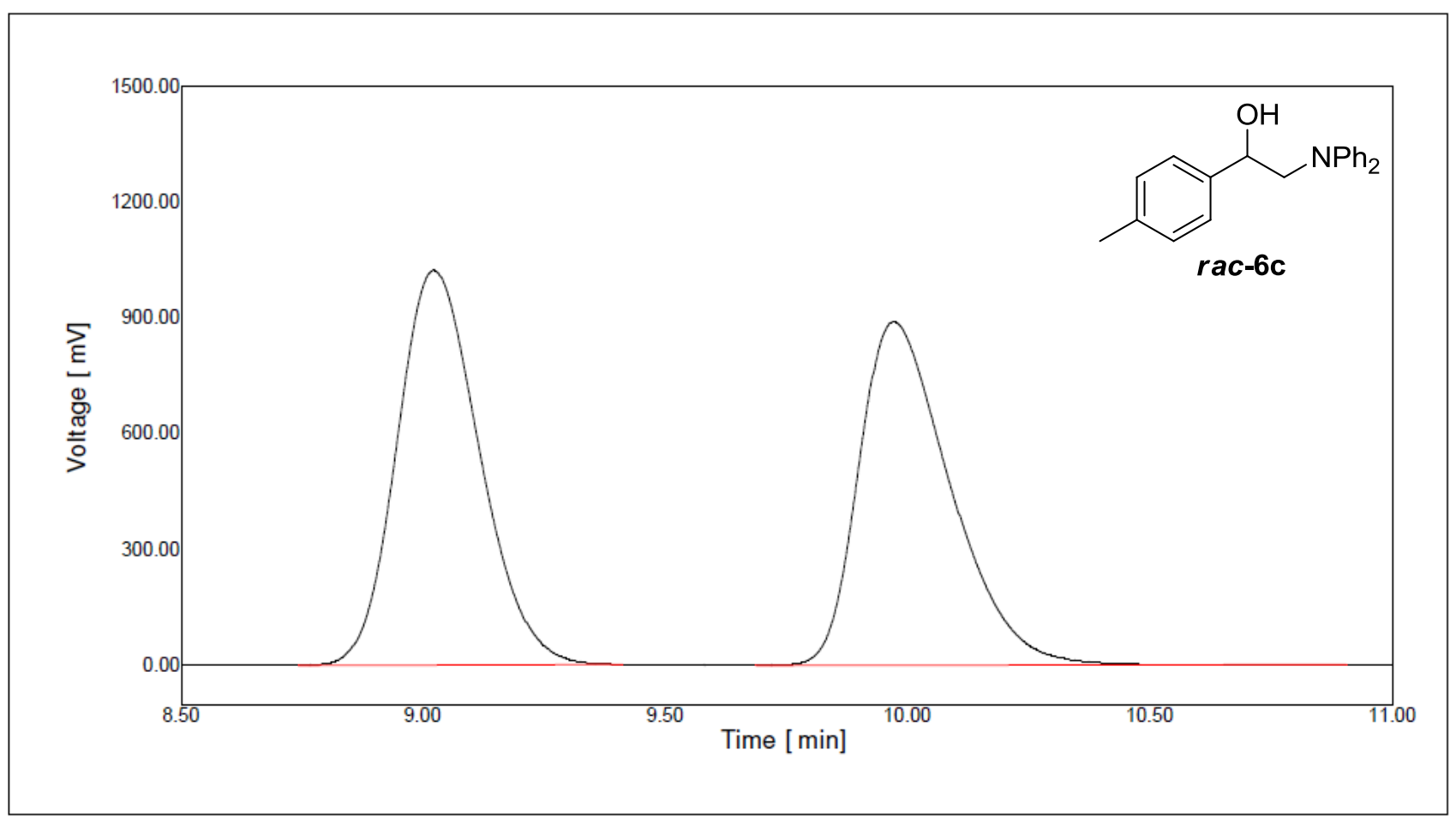

\begin{tabular}{|c|c|c|}
\hline No. & Time $(\mathrm{min})$ & Area ratio $(\%)$ \\
\hline 1 & 9.0233 & 50.94 \\
\hline 2 & 9.9717 & 49.06 \\
\hline
\end{tabular}

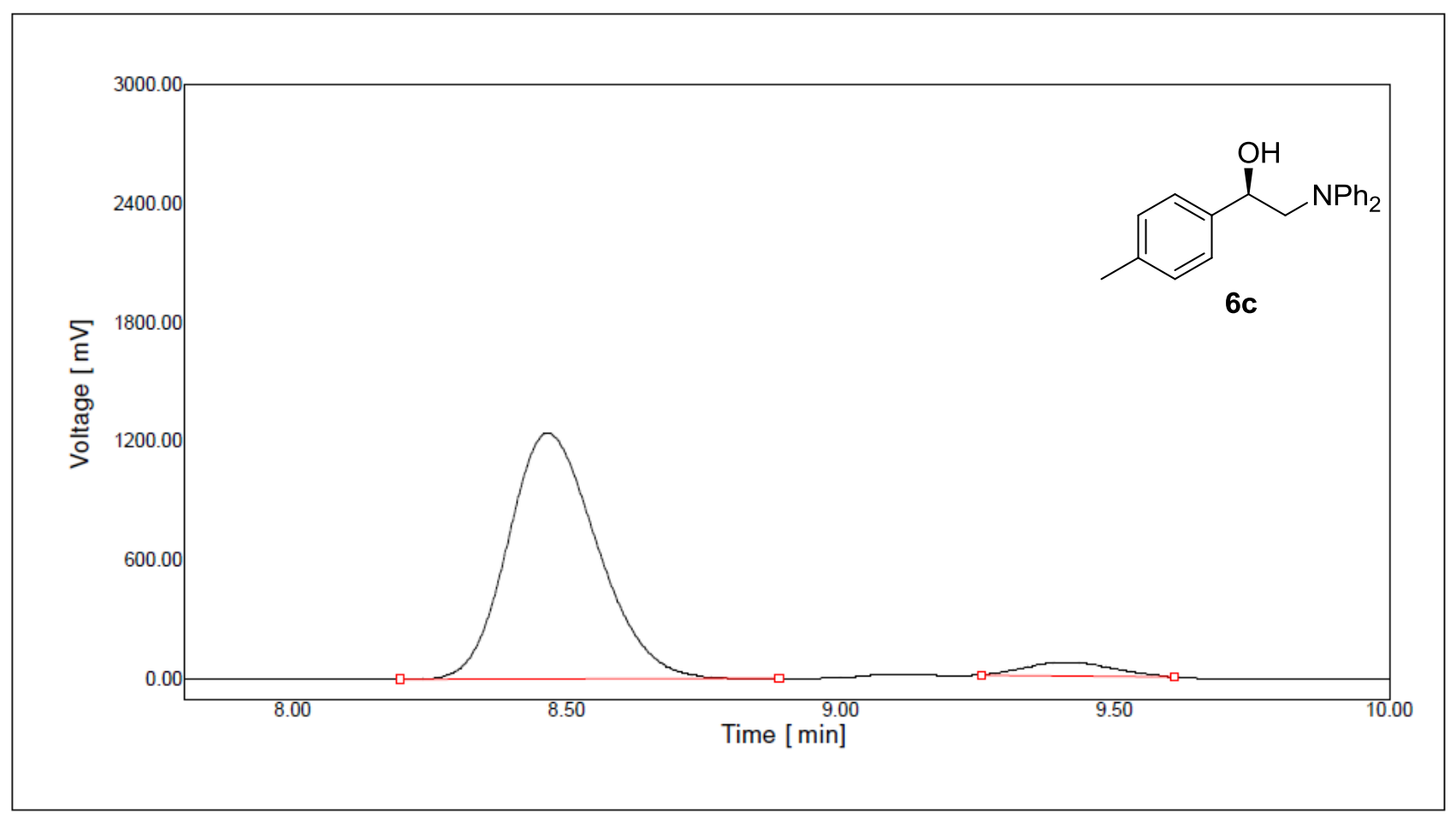

\begin{tabular}{|c|c|c|}
\hline No. & Time $(\mathrm{min})$ & Area ratio $(\%)$ \\
\hline 1 & 8.4650 & 94.91 \\
\hline 2 & 9.4100 & 5.09 \\
\hline
\end{tabular}




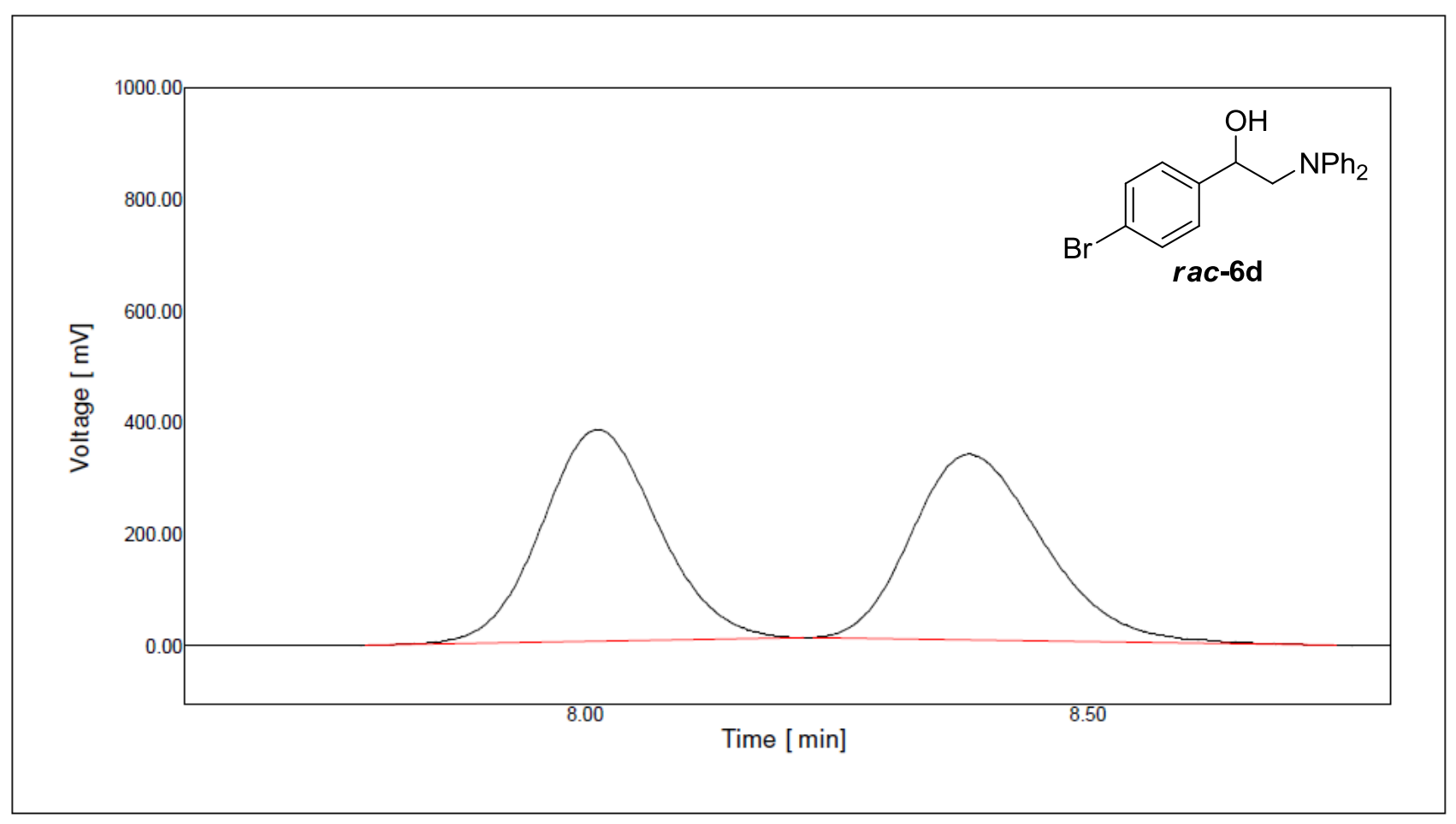

\begin{tabular}{|c|c|c|}
\hline No. & Time $(\mathrm{min})$ & Area ratio $(\%)$ \\
\hline 1 & 8.0117 & 50.31 \\
\hline 2 & 8.3817 & 49.69 \\
\hline
\end{tabular}

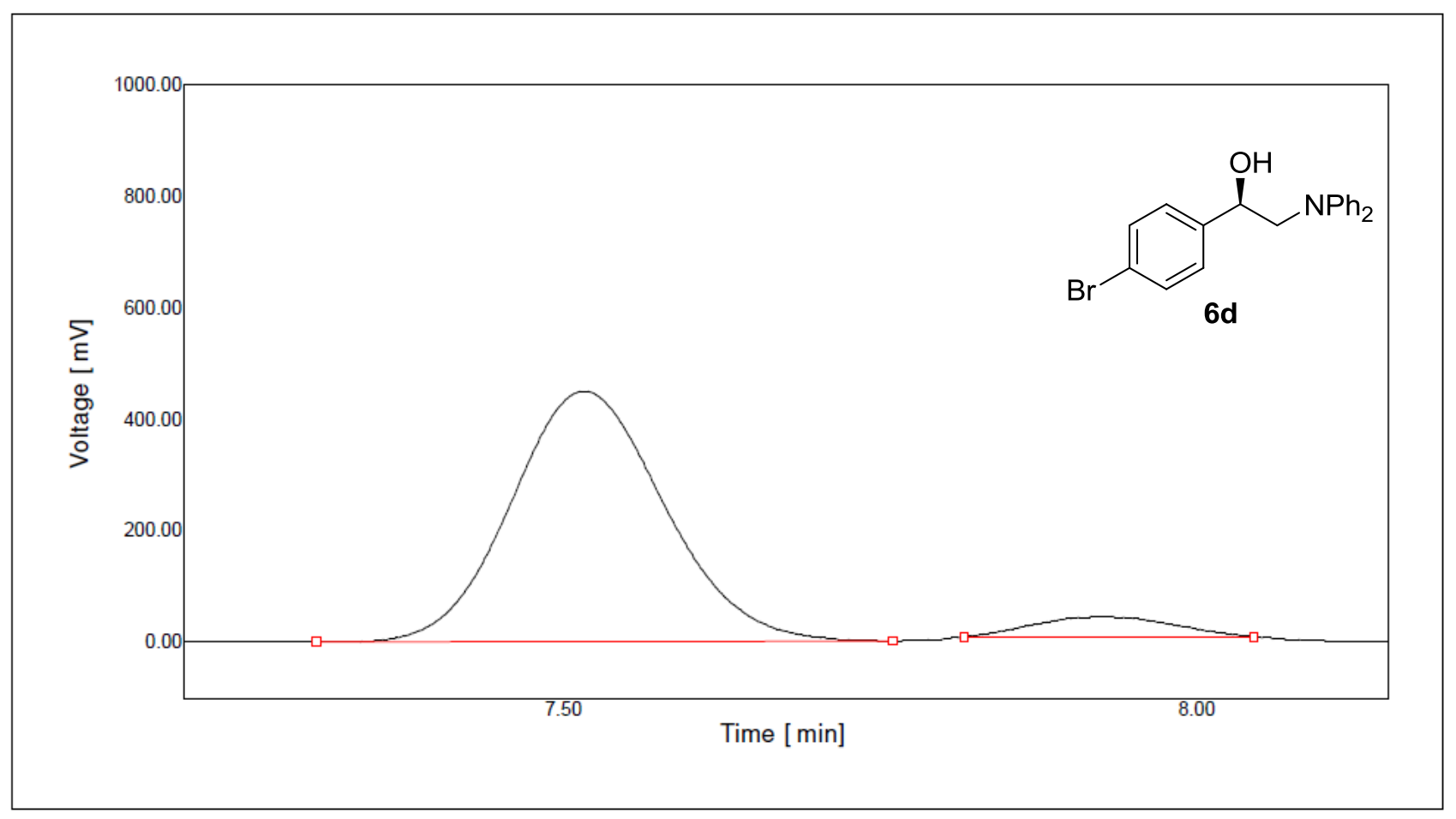

\begin{tabular}{|c|c|c|}
\hline No. & Time $(\mathrm{min})$ & Area ratio (\%) \\
\hline 1 & 7.5167 & 93.19 \\
\hline 2 & 7.9250 & 6.81 \\
\hline
\end{tabular}




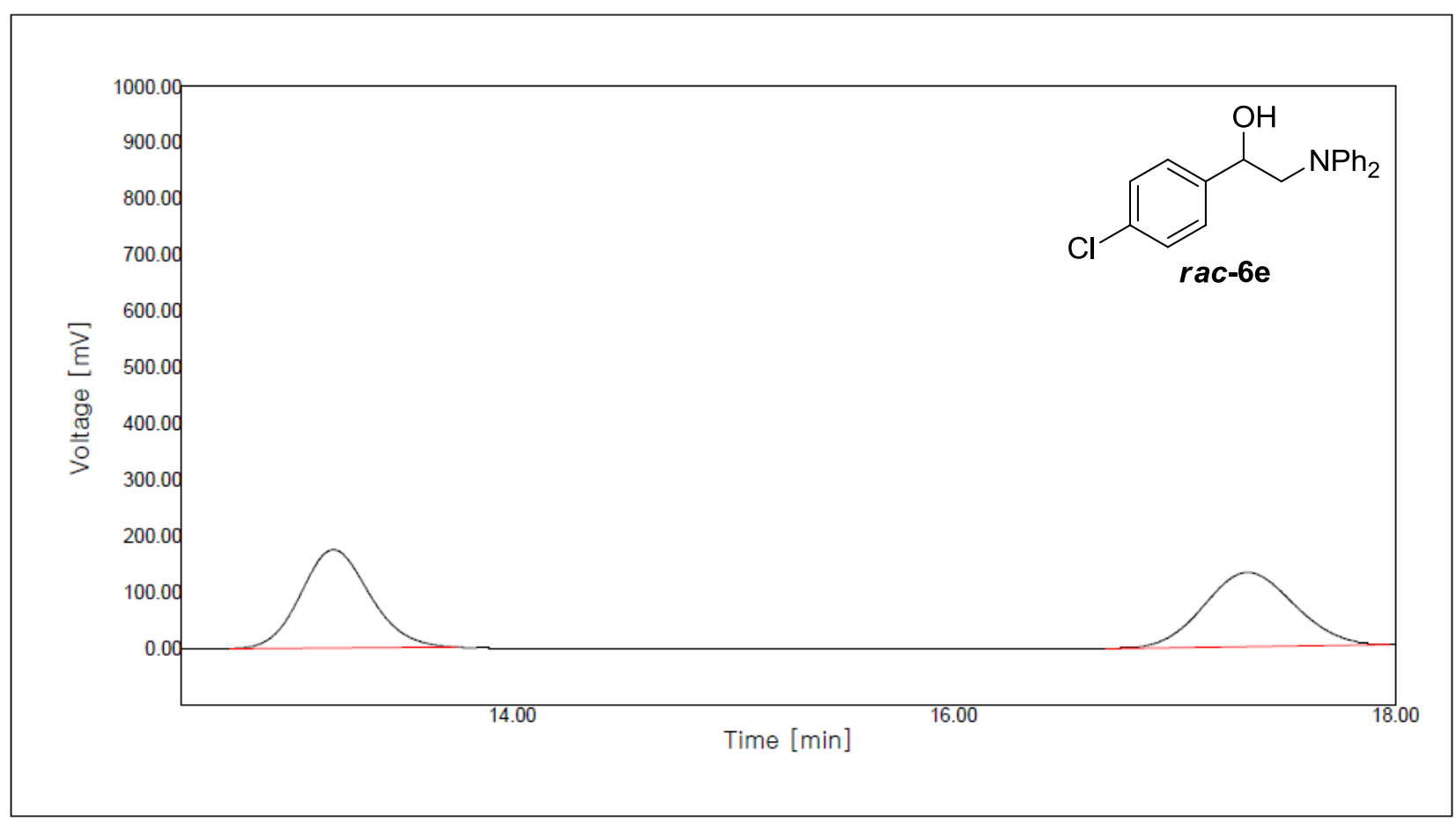

\begin{tabular}{|c|c|c|}
\hline No. & Time(min) & Area ratio (\%) \\
\hline 1 & 13.1883 & 50.27 \\
\hline 2 & 17.3333 & 49.73 \\
\hline
\end{tabular}

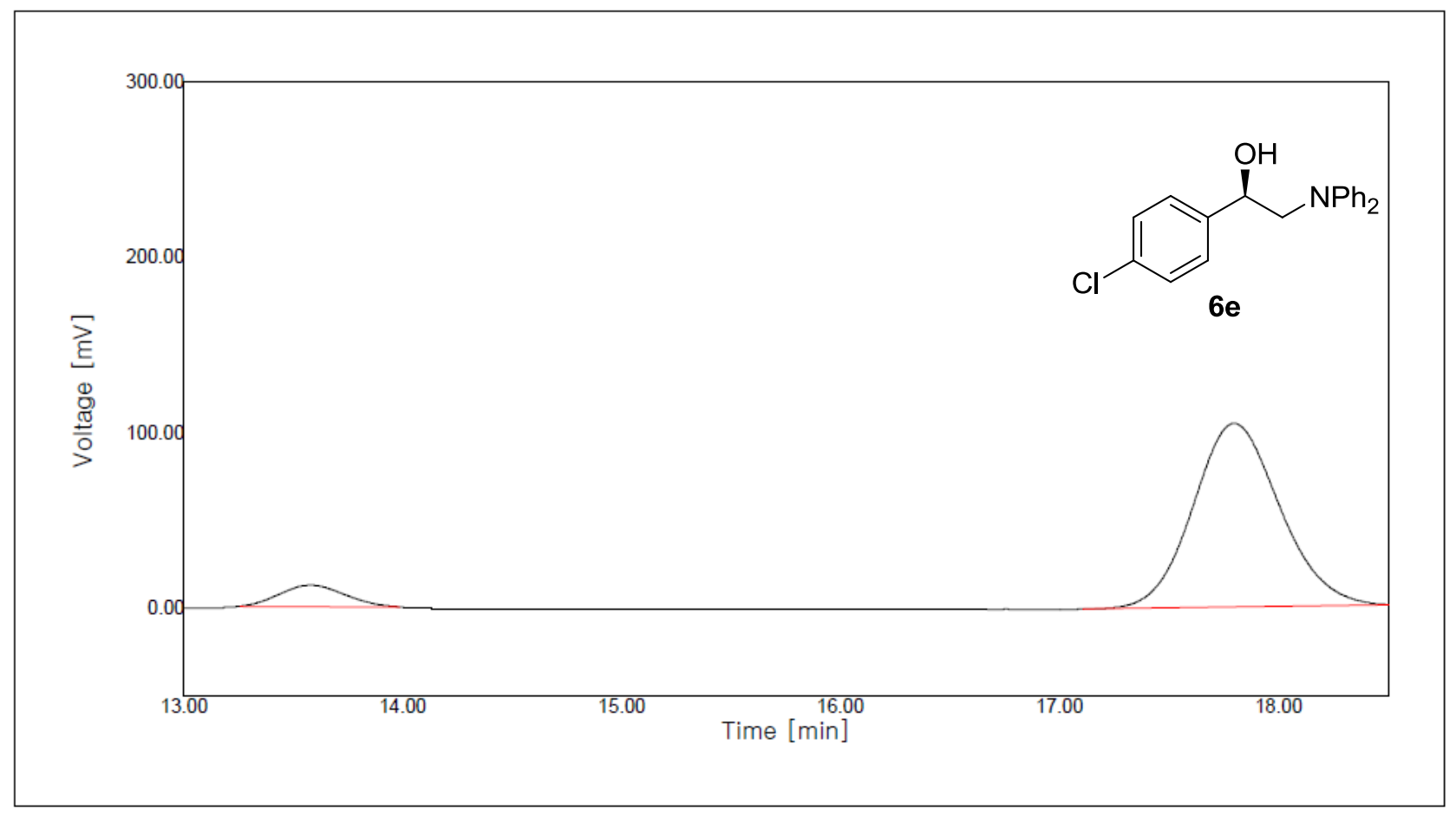

\begin{tabular}{|r|r|r|}
\hline No. & Time $(\mathrm{min})$ & \multicolumn{1}{|c|}{ Area ratio (\%) } \\
\hline 1 & & 13.5800 \\
\hline 2 & 17.7983 & 91.03 \\
\hline
\end{tabular}




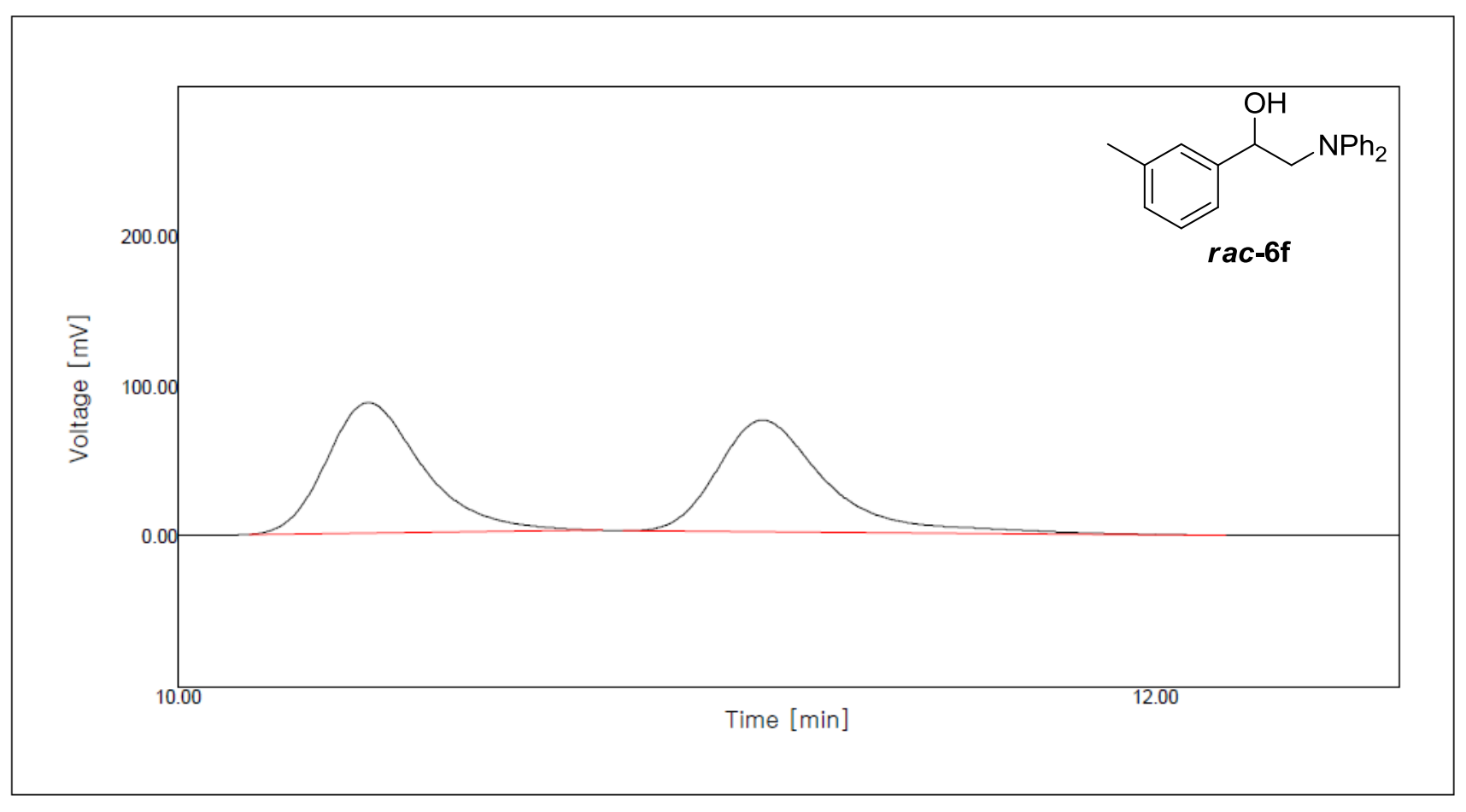

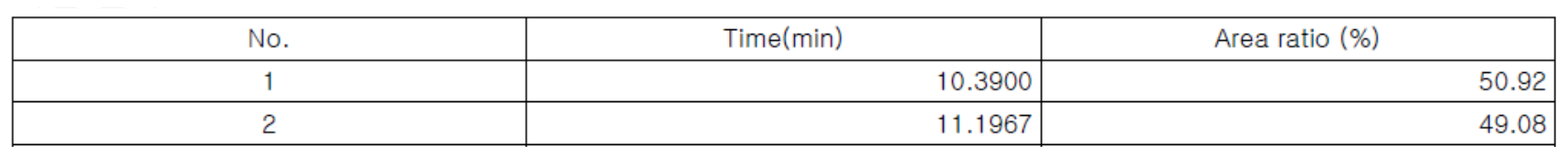

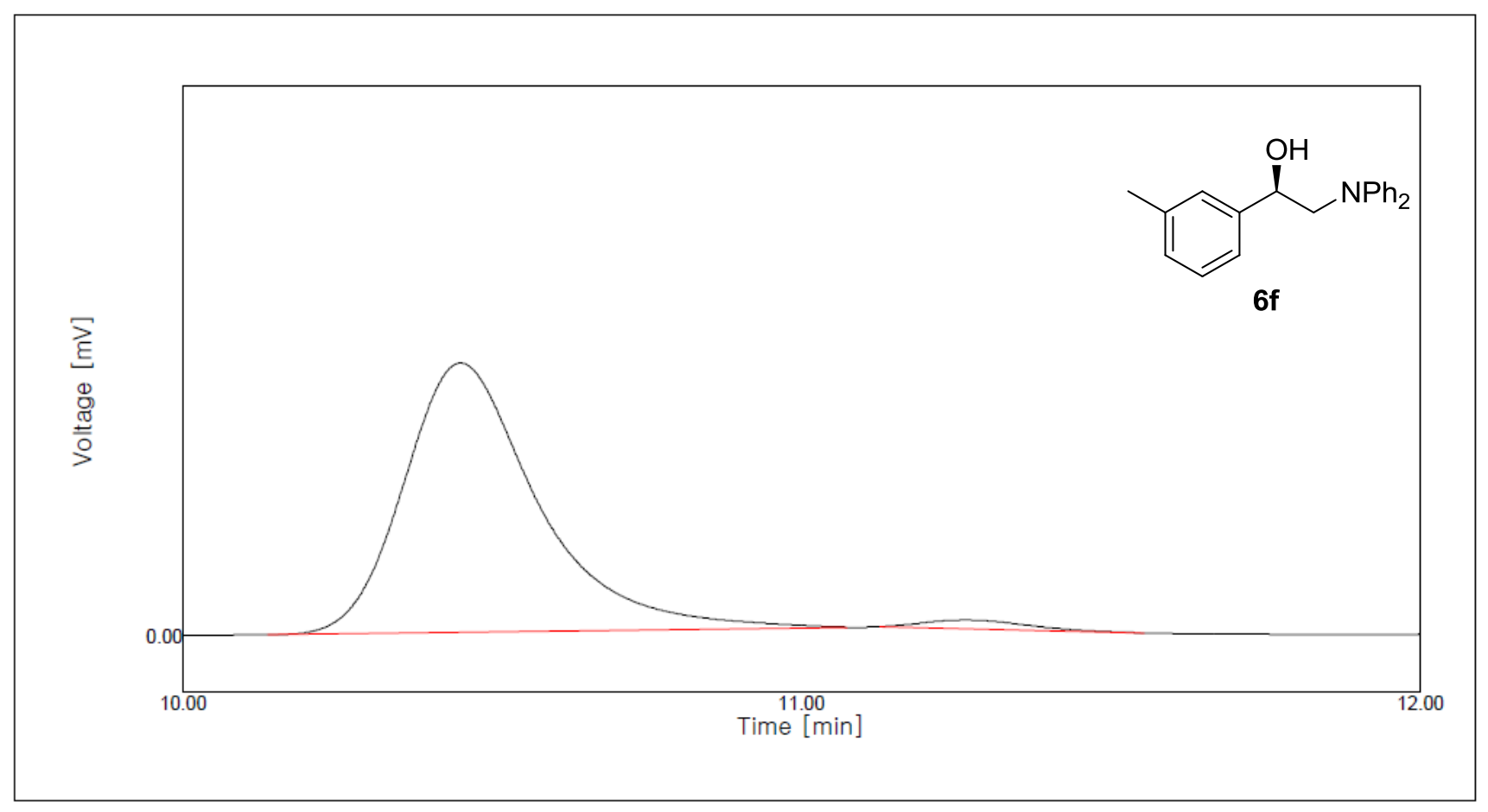

\begin{tabular}{|c|c|c|}
\hline No. & Time(min) & Area ratio (\%) \\
\hline 1 & 10.4500 & 97.59 \\
\hline 2 & 11.2650 & 2.41 \\
\hline
\end{tabular}




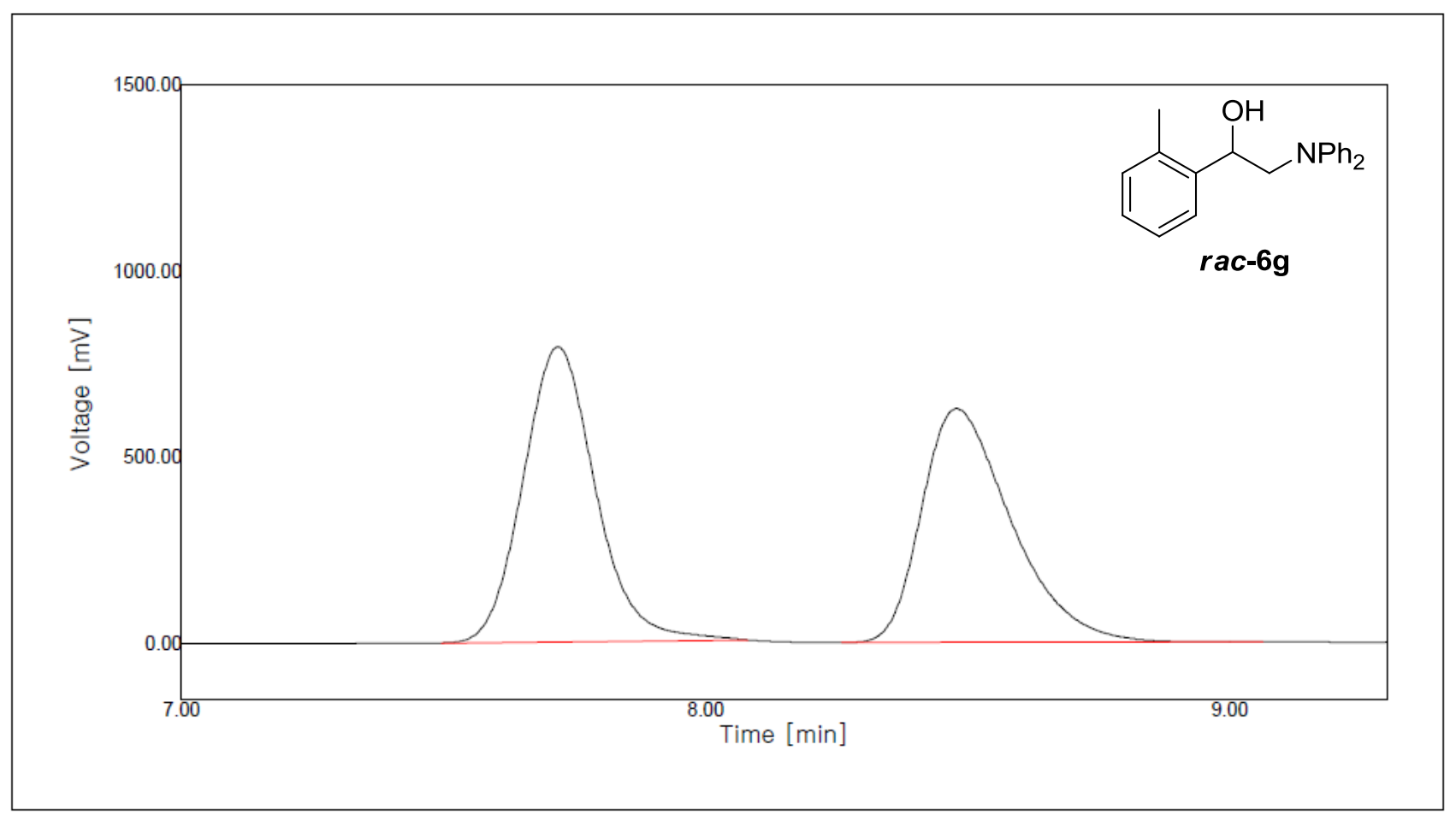

\begin{tabular}{|c|c|c|}
\hline No. & Time(min) & Area ratio (\%) \\
\hline 1 & 7.7183 & 50.50 \\
\hline 2 & 8.4800 & 49.50 \\
\hline
\end{tabular}

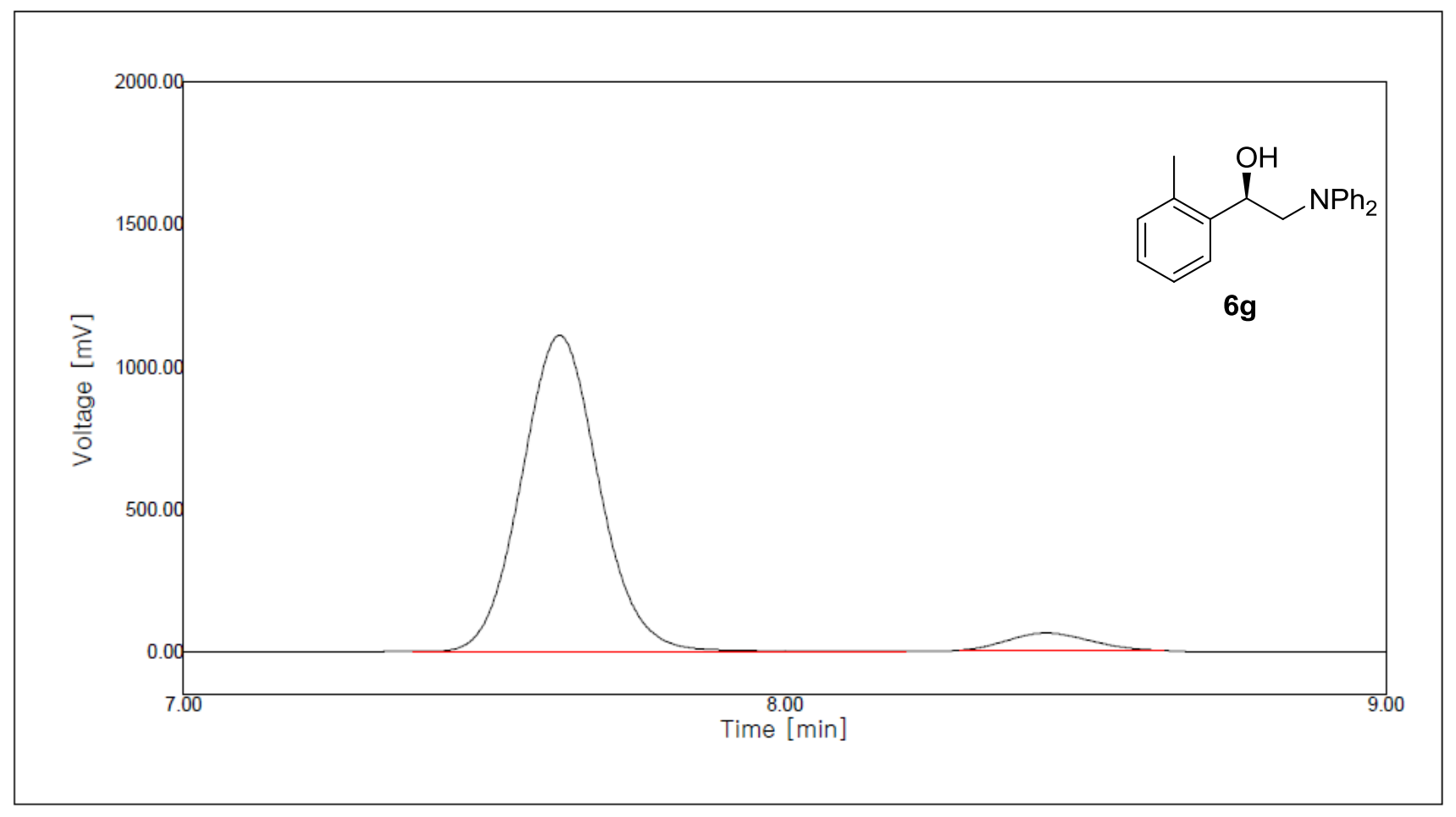

\begin{tabular}{|r|r|r|}
\hline No. & Time $(\mathrm{min})$ & \multicolumn{1}{|c|}{ Area ratio (\%) } \\
\hline 1 & & 7.6267 \\
\hline 2 & 8.4350 & 5.49 \\
\hline
\end{tabular}




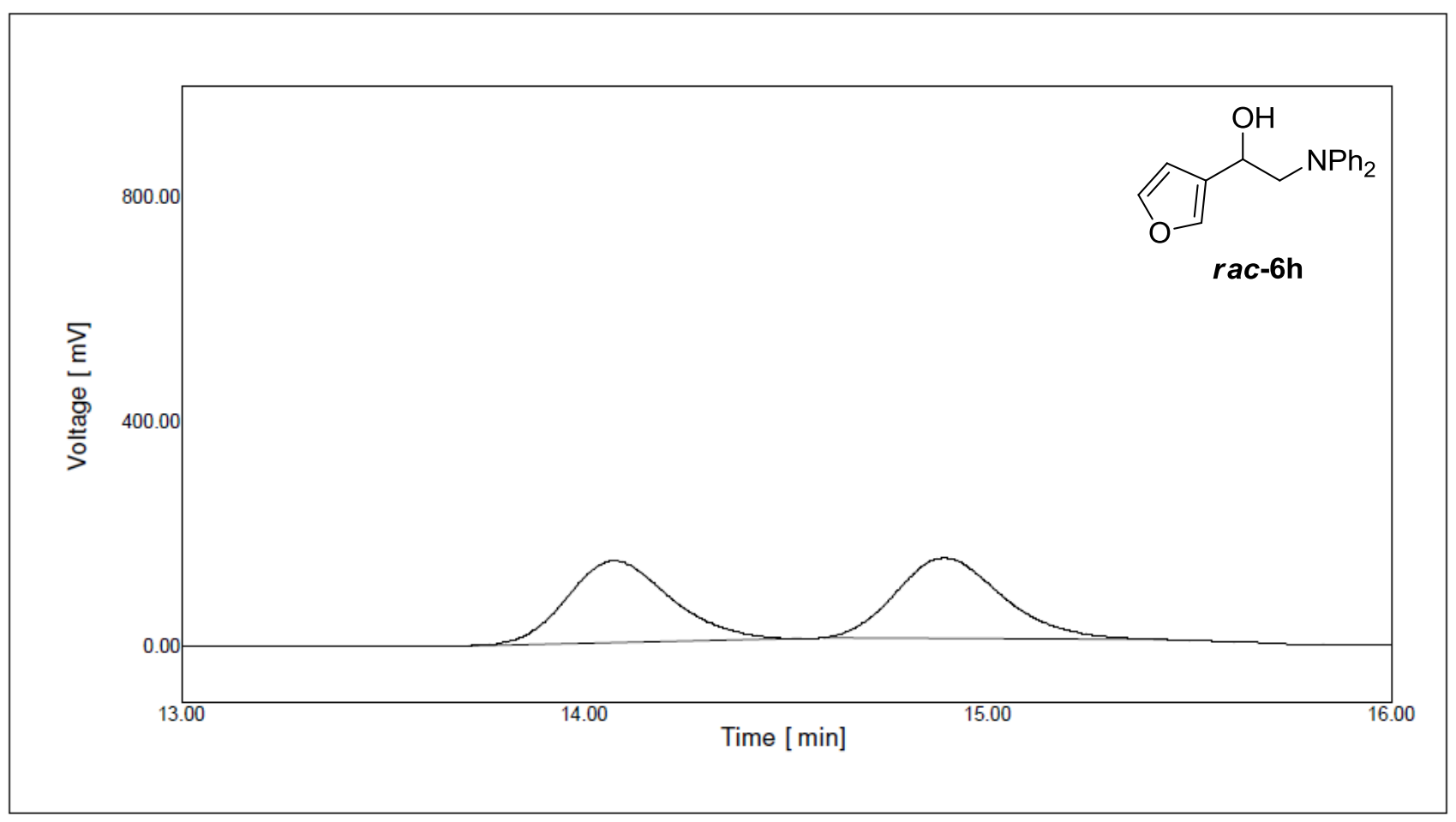

\begin{tabular}{|c|c|c|}
\hline Peak \# & Time (min) & Area $(\%)$ \\
\hline 1 & 14.0750 & 49.62 \\
\hline 2 & 14.8917 & 50.38 \\
\hline
\end{tabular}

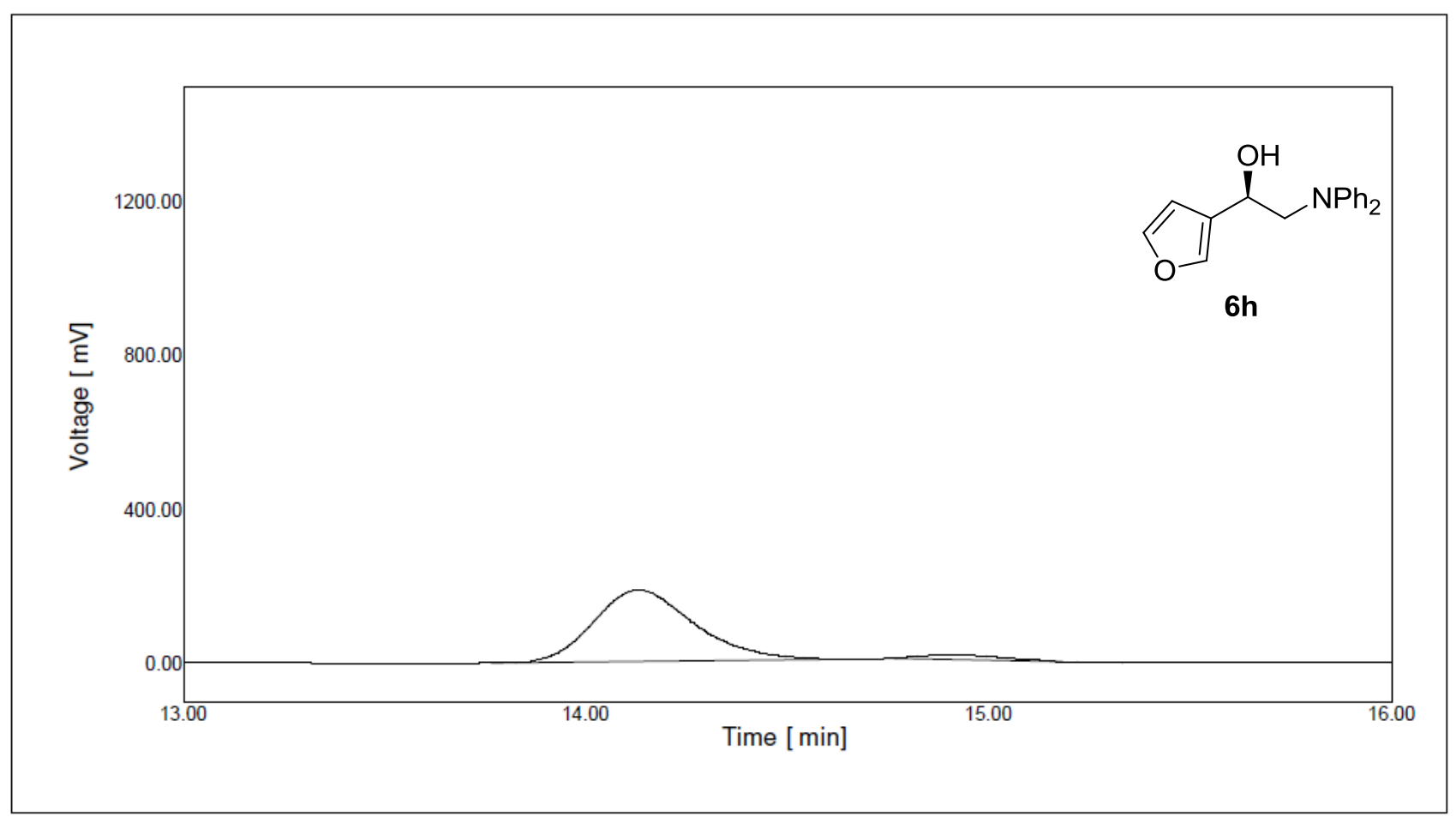

\begin{tabular}{|c|r|r|}
\hline Peak\# & Time $(\mathrm{min})$ & \multicolumn{1}{|c|}{ Area (\%) } \\
\hline 1 & & 14.1317 \\
\hline 2 & 14.9083 & 54.63 \\
\hline
\end{tabular}




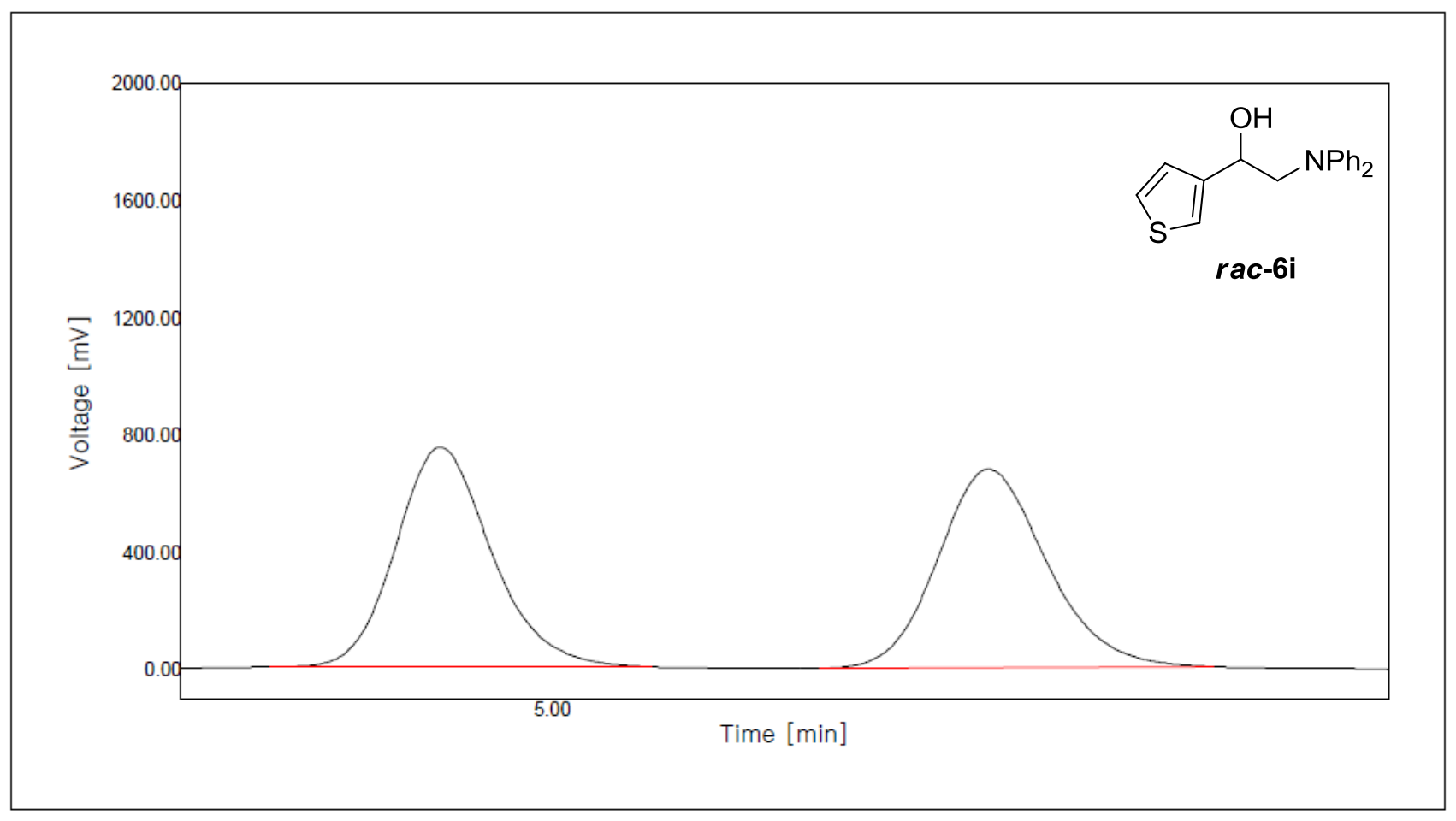

\begin{tabular}{|r|r|r|}
\hline No. & Time $(\mathrm{min})$ & \multicolumn{1}{|c|}{ Area ratio (\%) } \\
\hline 1 & & 4.8800 \\
\hline 2 & 5.4700 & 50.37 \\
\hline
\end{tabular}

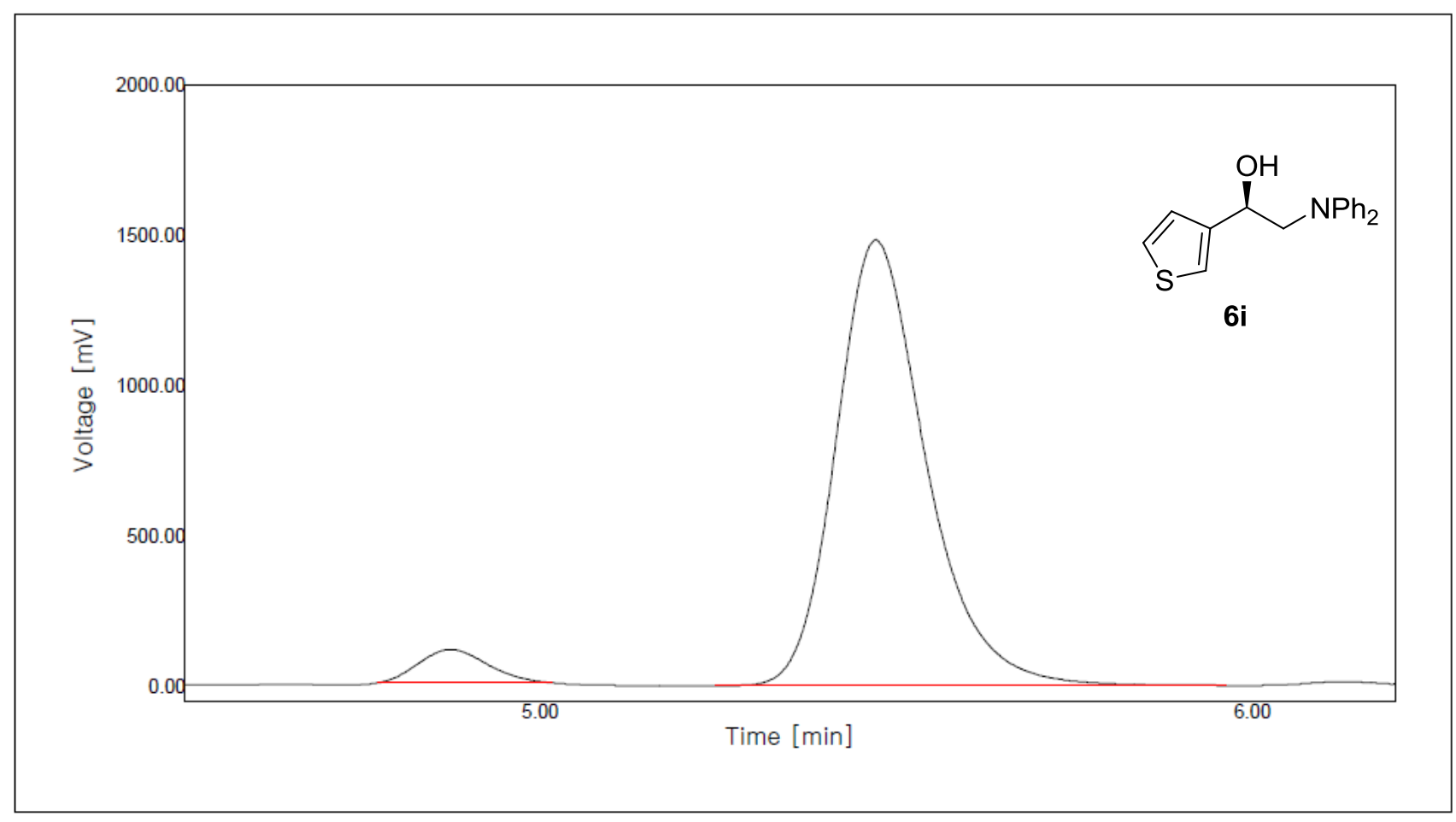

\begin{tabular}{|r|r|r|}
\hline No. & Time $(\mathrm{min})$ & \multicolumn{1}{|c|}{ Area ratio (\%) } \\
\hline 1 & & 5.40 \\
\hline 2 & 4.8733 & 94.60 \\
\hline
\end{tabular}




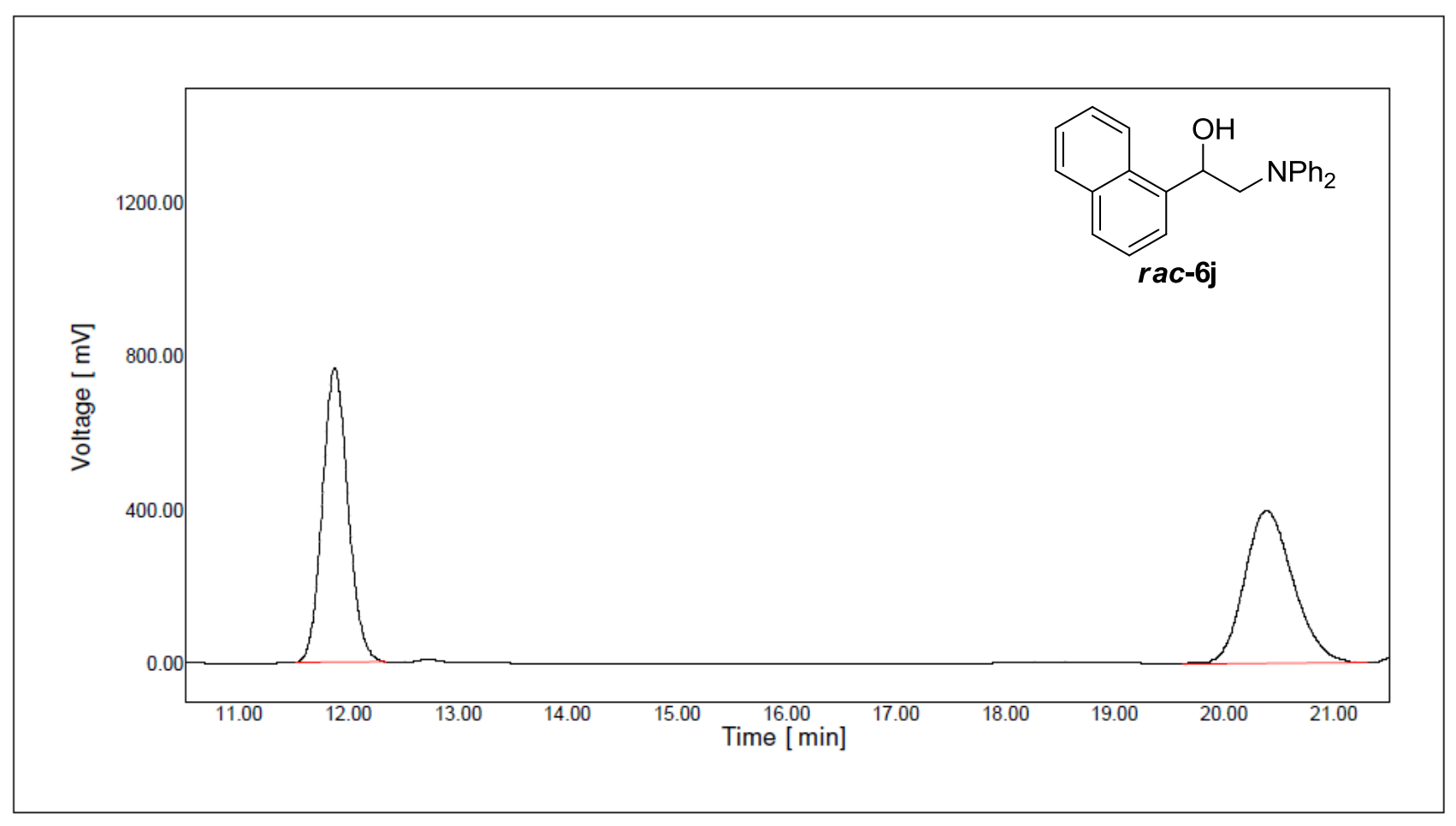

\begin{tabular}{|c|c|c|}
\hline Peak \# & Time (min) & Area (\%) \\
\hline 1 & 11.8733 & 50.49 \\
\hline 2 & 20.3850 & 49.51 \\
\hline
\end{tabular}

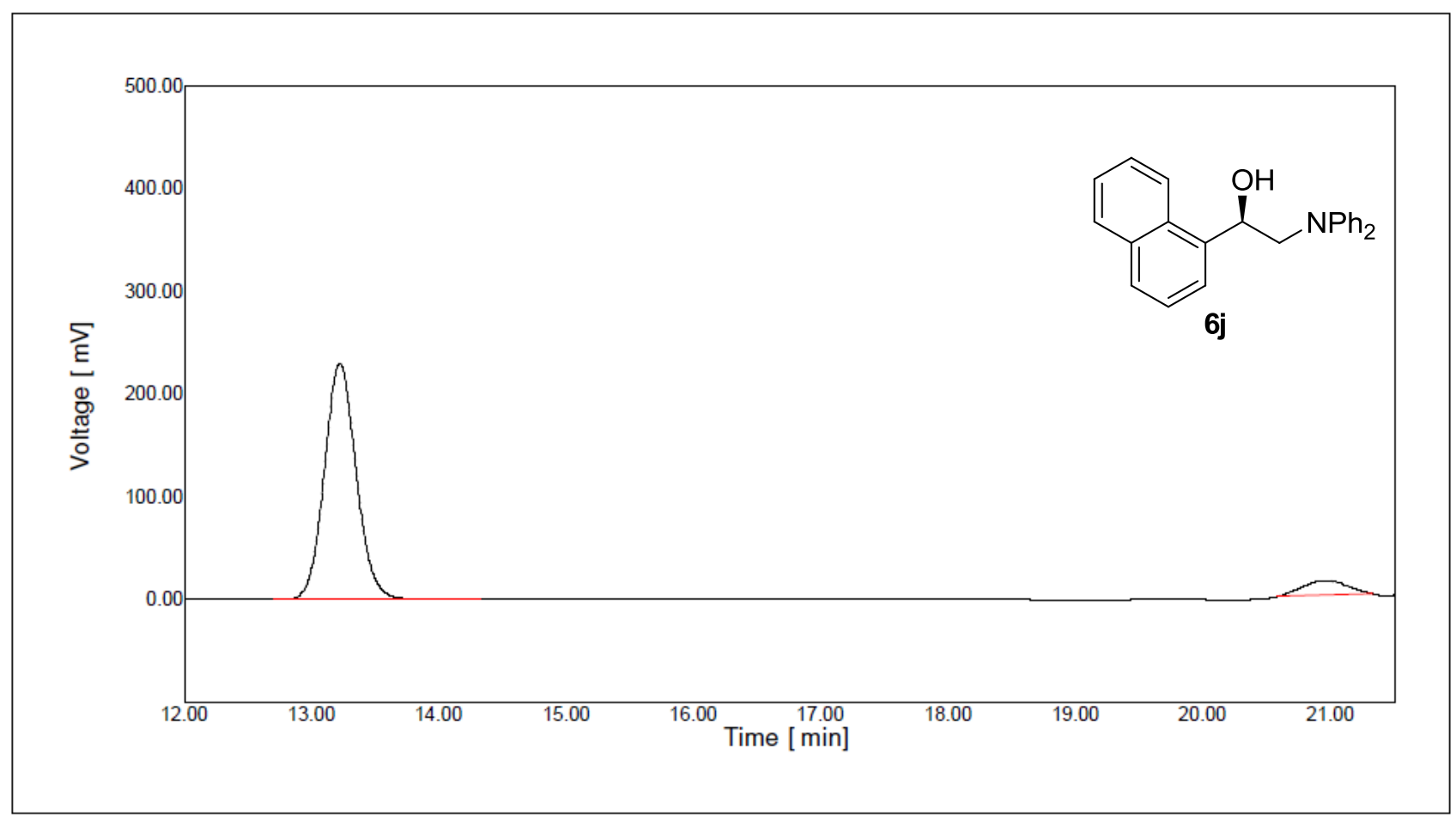

\begin{tabular}{|c|r|r|}
\hline Peak\# & Time $(\mathrm{min})$ & \multicolumn{1}{|c|}{ Area (\%) } \\
\hline 1 & & 13.2233 \\
\hline 2 & 20.9650 & 7.90 \\
\hline
\end{tabular}




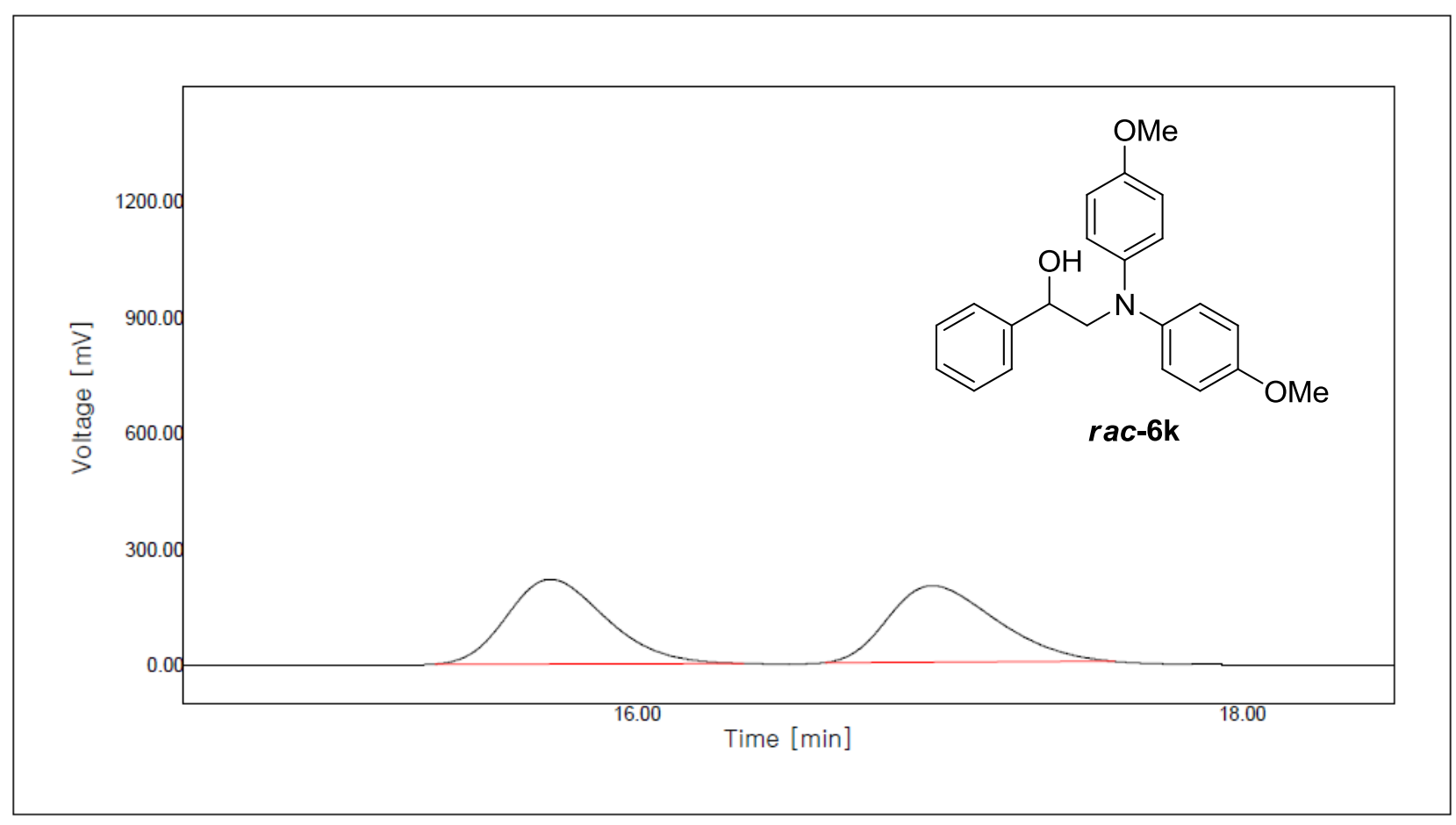

\begin{tabular}{|c|c|c|}
\hline No. & Time(min) & Area ratio (\%) \\
\hline 1 & 15.7133 & 50.39 \\
\hline 2 & 16.9750 & 49.61 \\
\hline
\end{tabular}

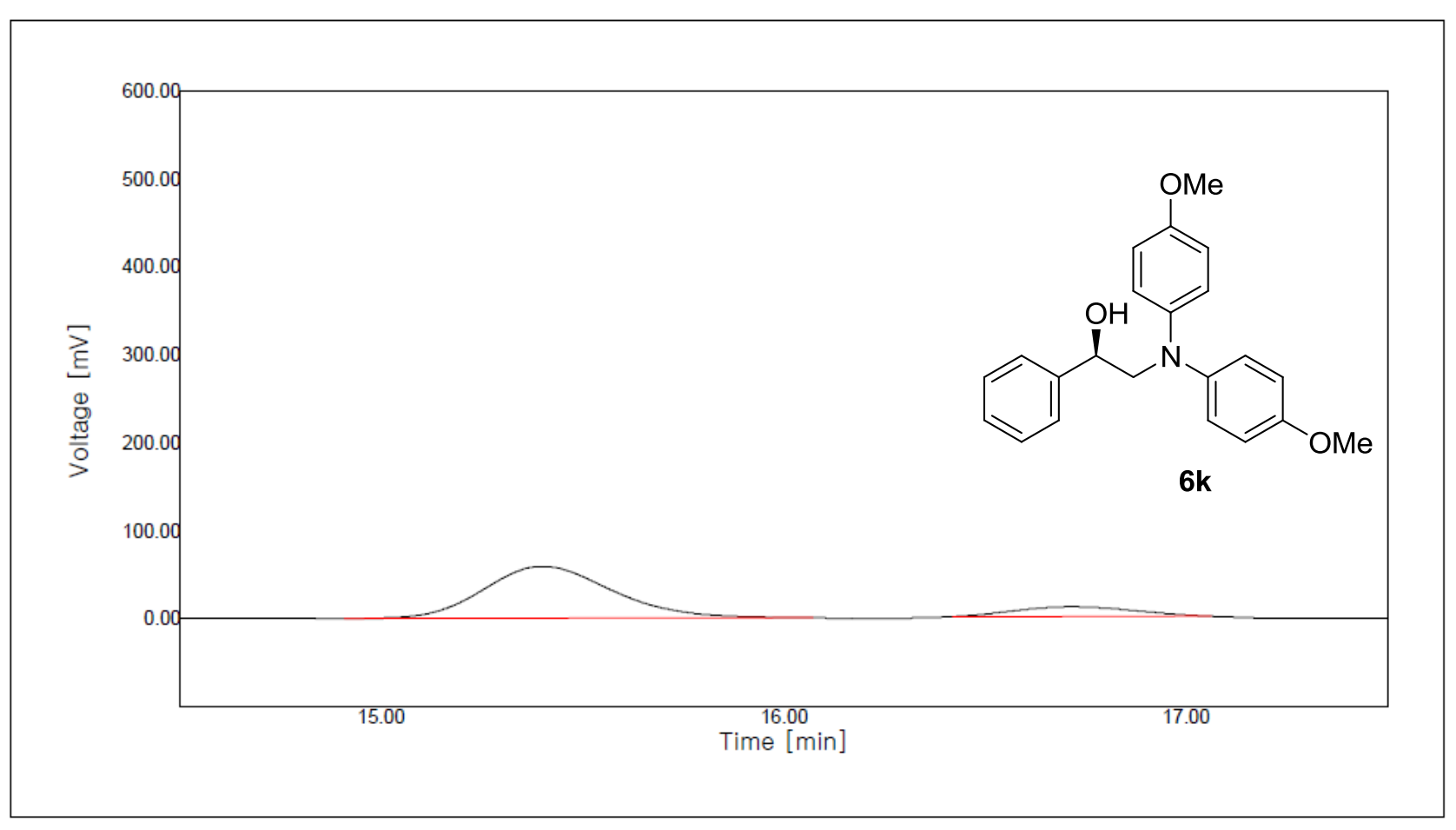

\begin{tabular}{|r|r|r|}
\hline No. & Time $(\mathrm{min})$ & \multicolumn{1}{|c|}{ Area ratio (\%) } \\
\hline 1 & & 15.4000 \\
\hline 2 & 16.7133 & 14.12 \\
\hline
\end{tabular}




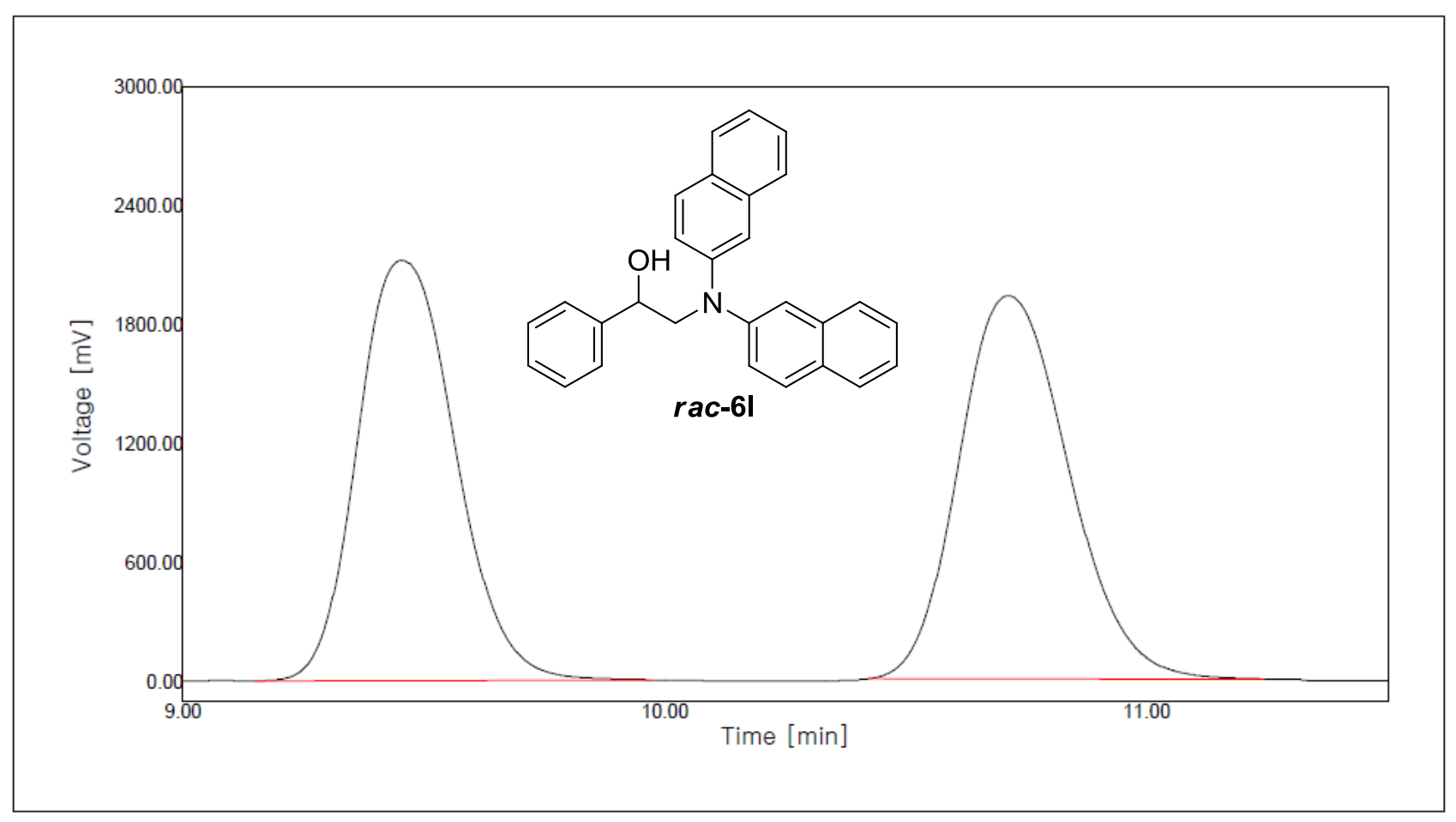

\begin{tabular}{|r|r|r|}
\hline No. & Time $(\mathrm{min})$ & \multicolumn{1}{|c|}{ Area ratio (\%) } \\
\hline 1 & & 9.4550 \\
\hline 2 & 10.7133 & 59.32 \\
\hline
\end{tabular}

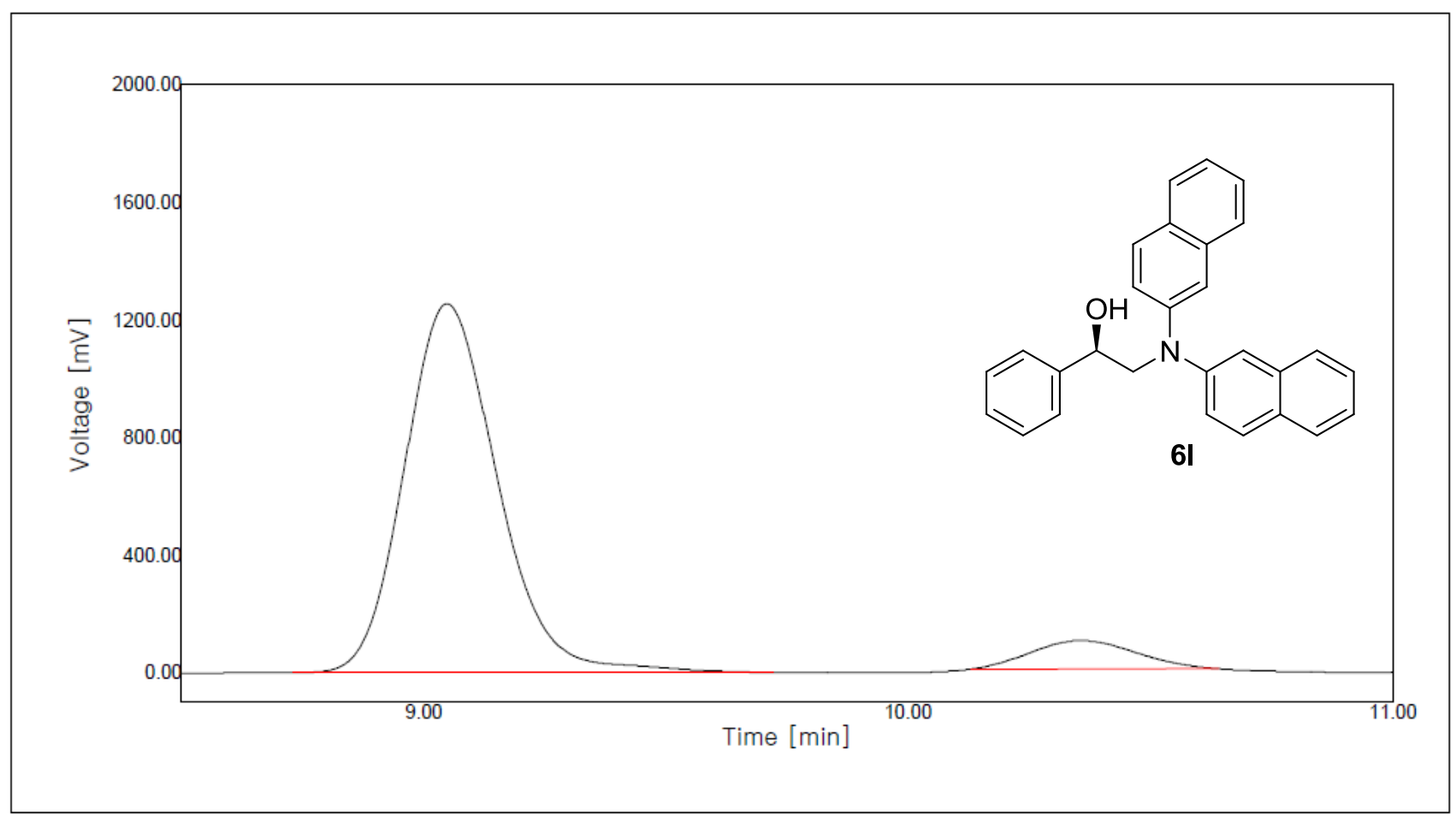

\begin{tabular}{|r|r|r|}
\hline No. & Time(min) & \multicolumn{1}{|c|}{ Area ratio (\%) } \\
\hline 1 & & 91.97 \\
\hline 2 & & 10.0483 \\
8.03 \\
\hline
\end{tabular}




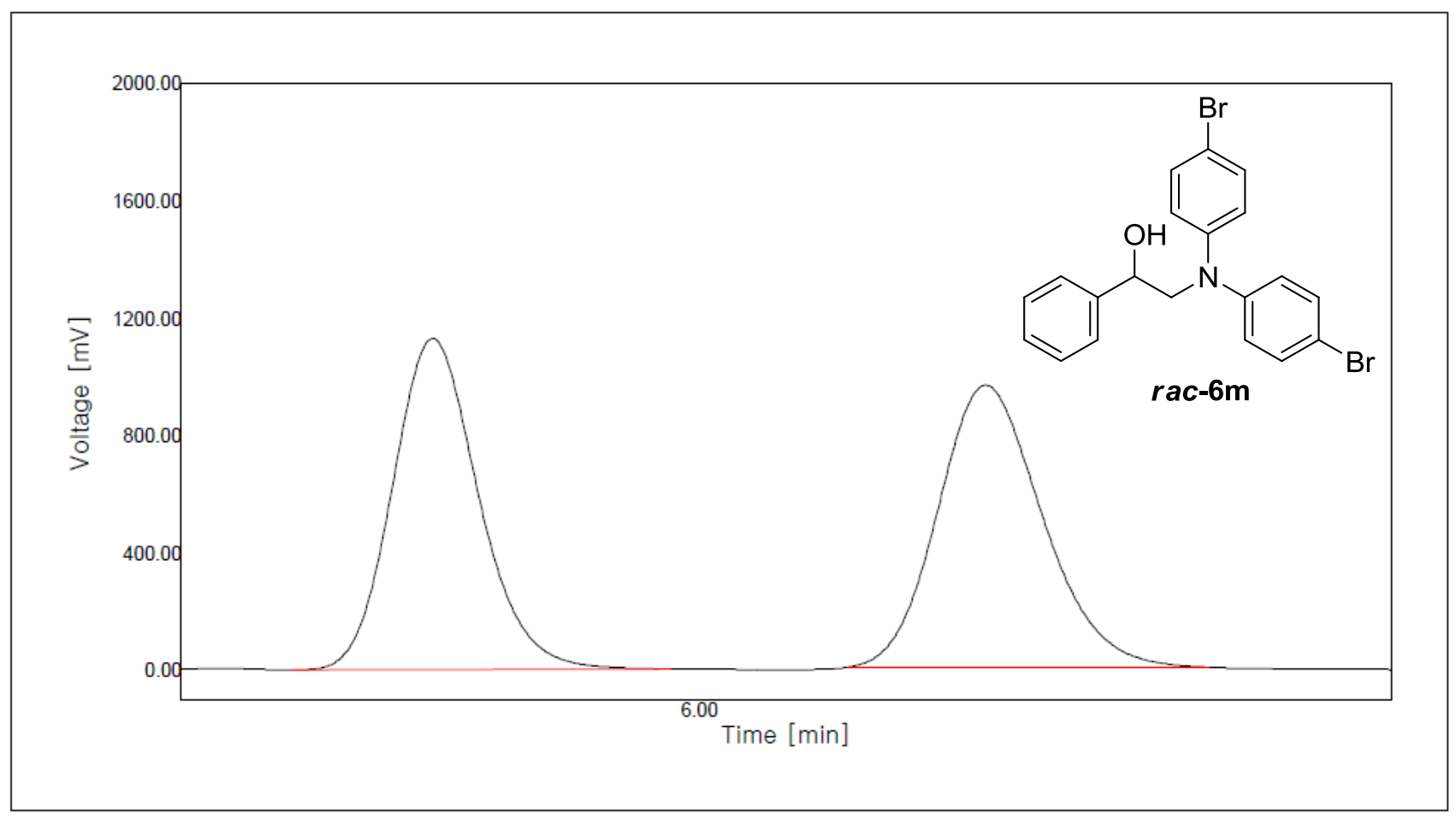

\begin{tabular}{|r|r|r|}
\hline No. & Time $(\mathrm{min})$ & \multicolumn{1}{|c|}{ Area ratio (\%) } \\
\hline 1 & & 5.6917 \\
\hline 2 & 6.3317 & 59.07 \\
\hline
\end{tabular}

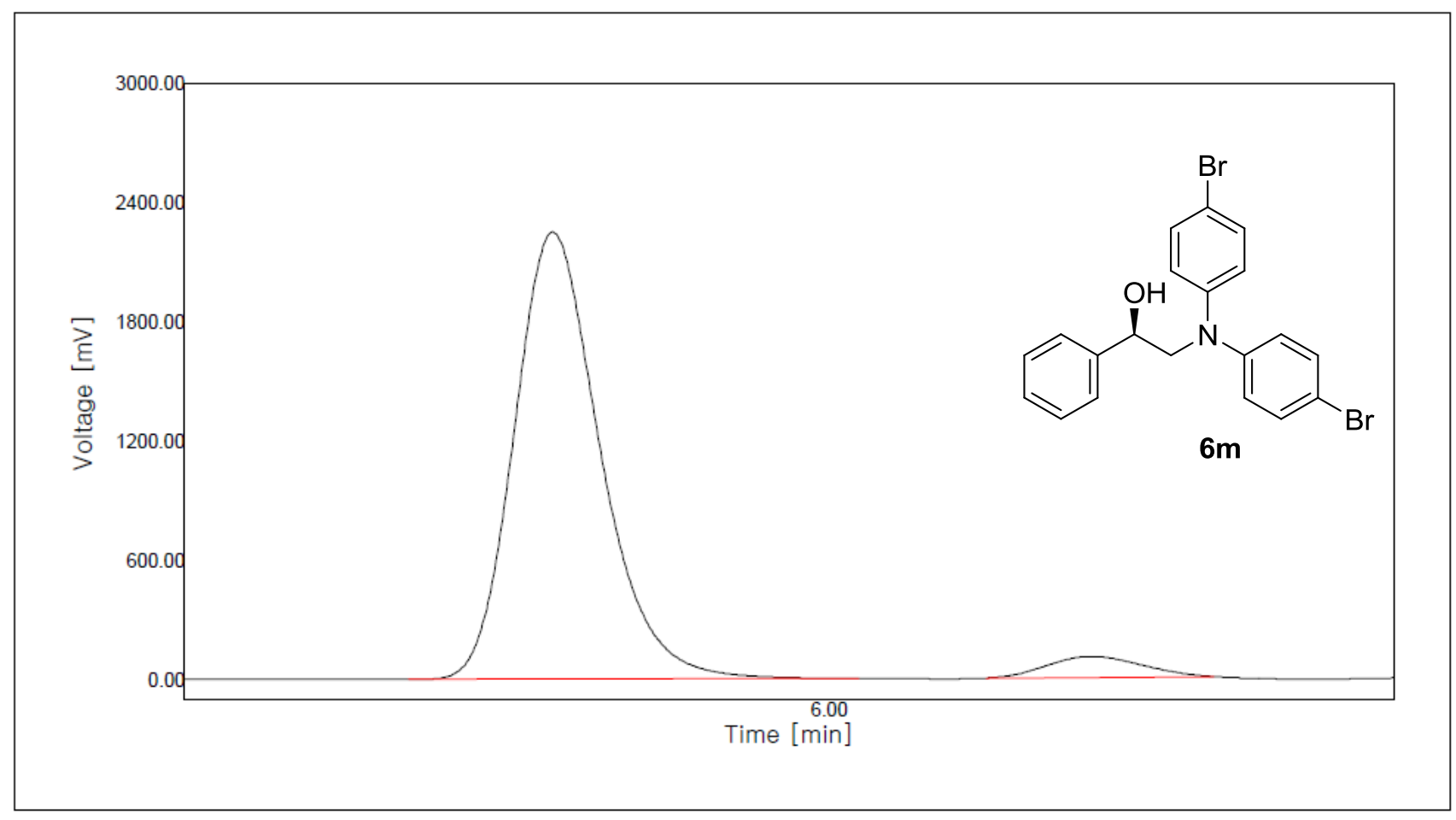

\begin{tabular}{|r|r|r|}
\hline No. & Time $(\mathrm{min})$ & \multicolumn{1}{|c|}{ Area ratio (\%) } \\
\hline 1 & & 5.6567 \\
\hline 2 & 6.3250 & 4.92 \\
\hline
\end{tabular}




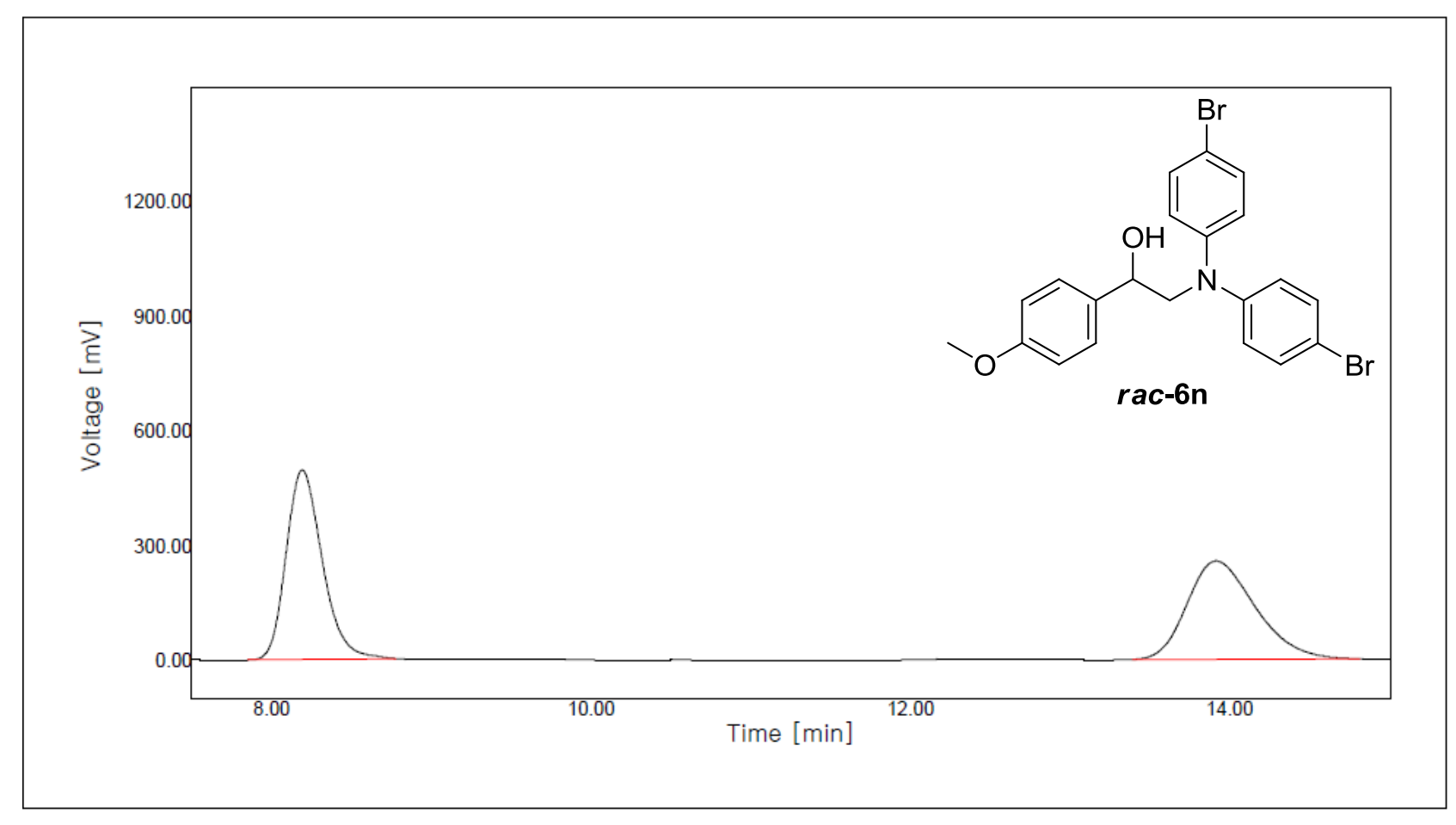

\begin{tabular}{|r|r|r|}
\hline No. & Time $(\mathrm{min})$ & \multicolumn{1}{|c|}{ Area ratio (\%) } \\
\hline 1 & 8.1933 & 50.53 \\
\hline 2 & 13.9117 & 49.47 \\
\hline
\end{tabular}

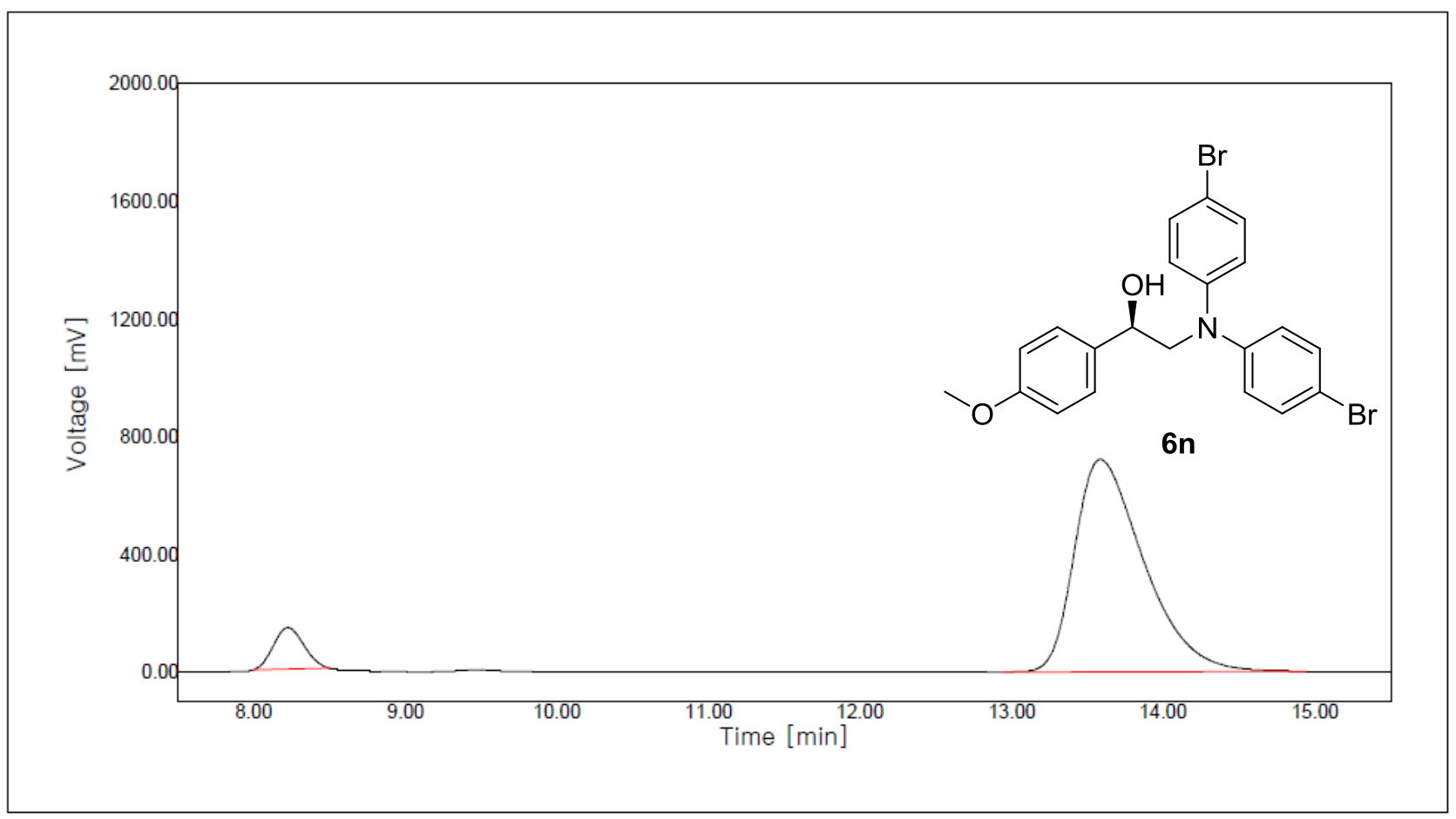

\begin{tabular}{|r|r|r|}
\hline No. & Time $(\mathrm{min})$ & \multicolumn{1}{|c|}{ Area ratio (\%) } \\
\hline 1 & & 8.2250 \\
\hline 2 & 13.5850 & 91.98 \\
\hline
\end{tabular}




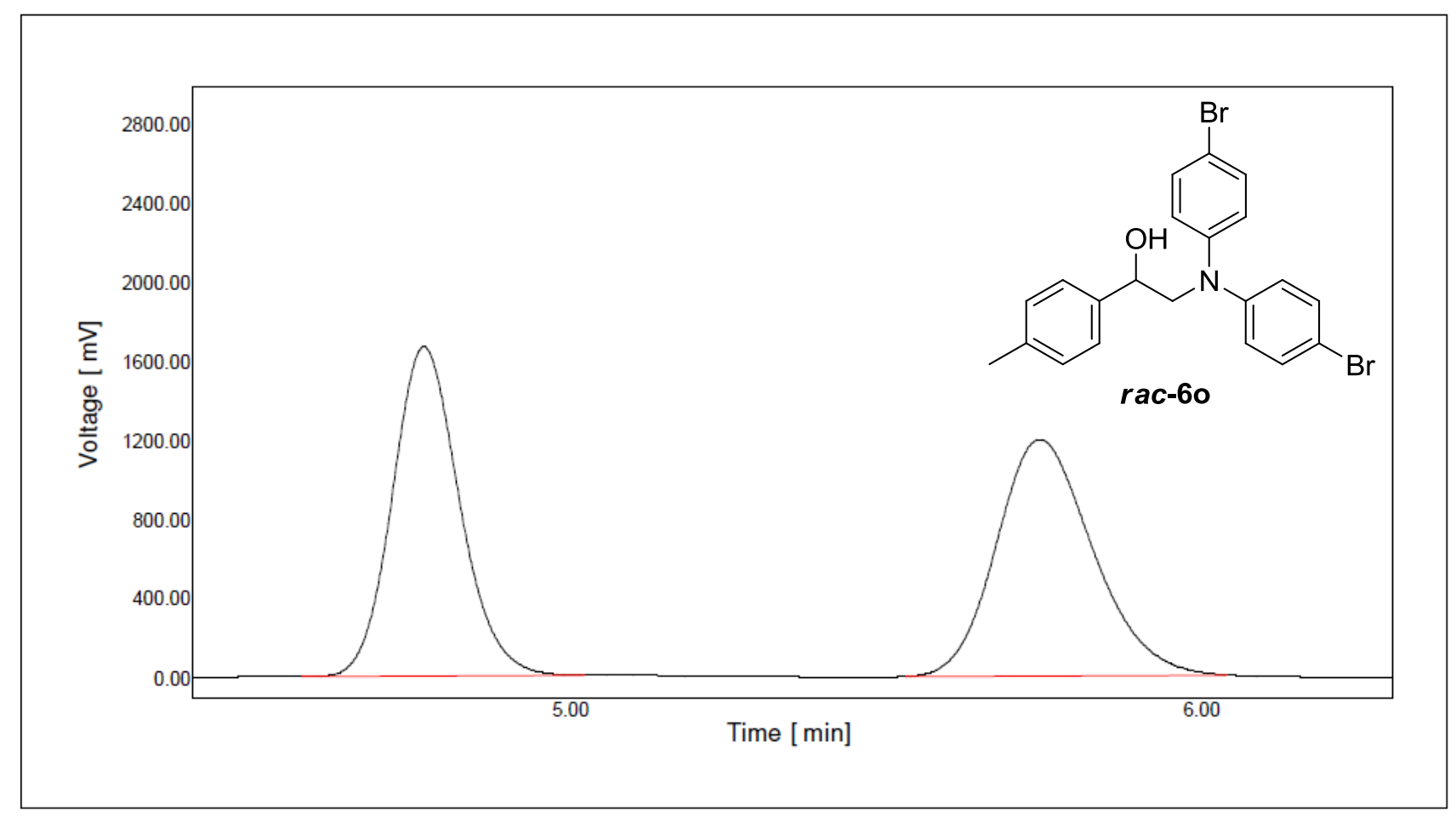

\begin{tabular}{|c|c|c|}
\hline Peak \# & Time (min) & Area (\%) \\
\hline 1 & 4.7683 & 49.23 \\
\hline 2 & 5.7433 & 50.77 \\
\hline
\end{tabular}

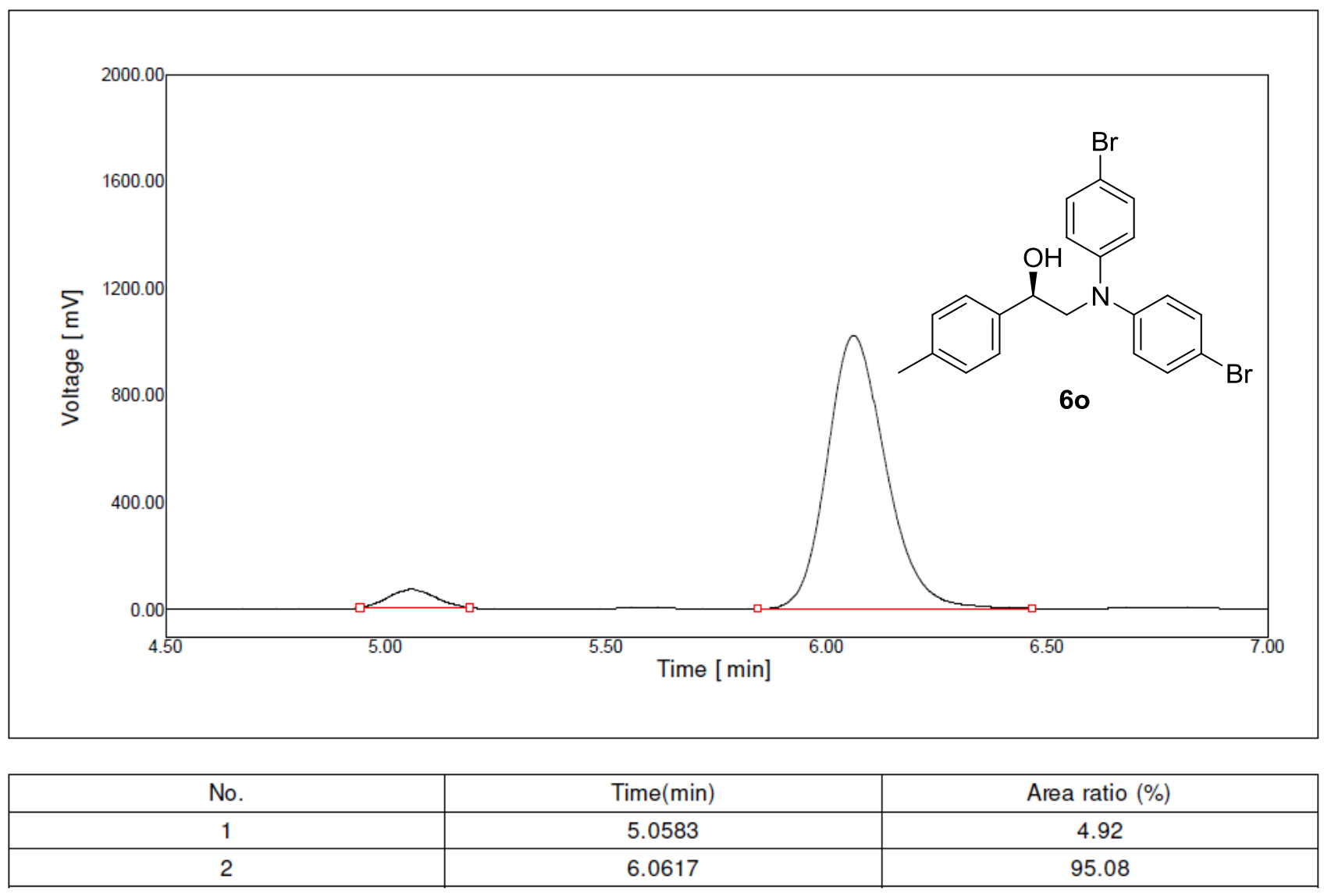




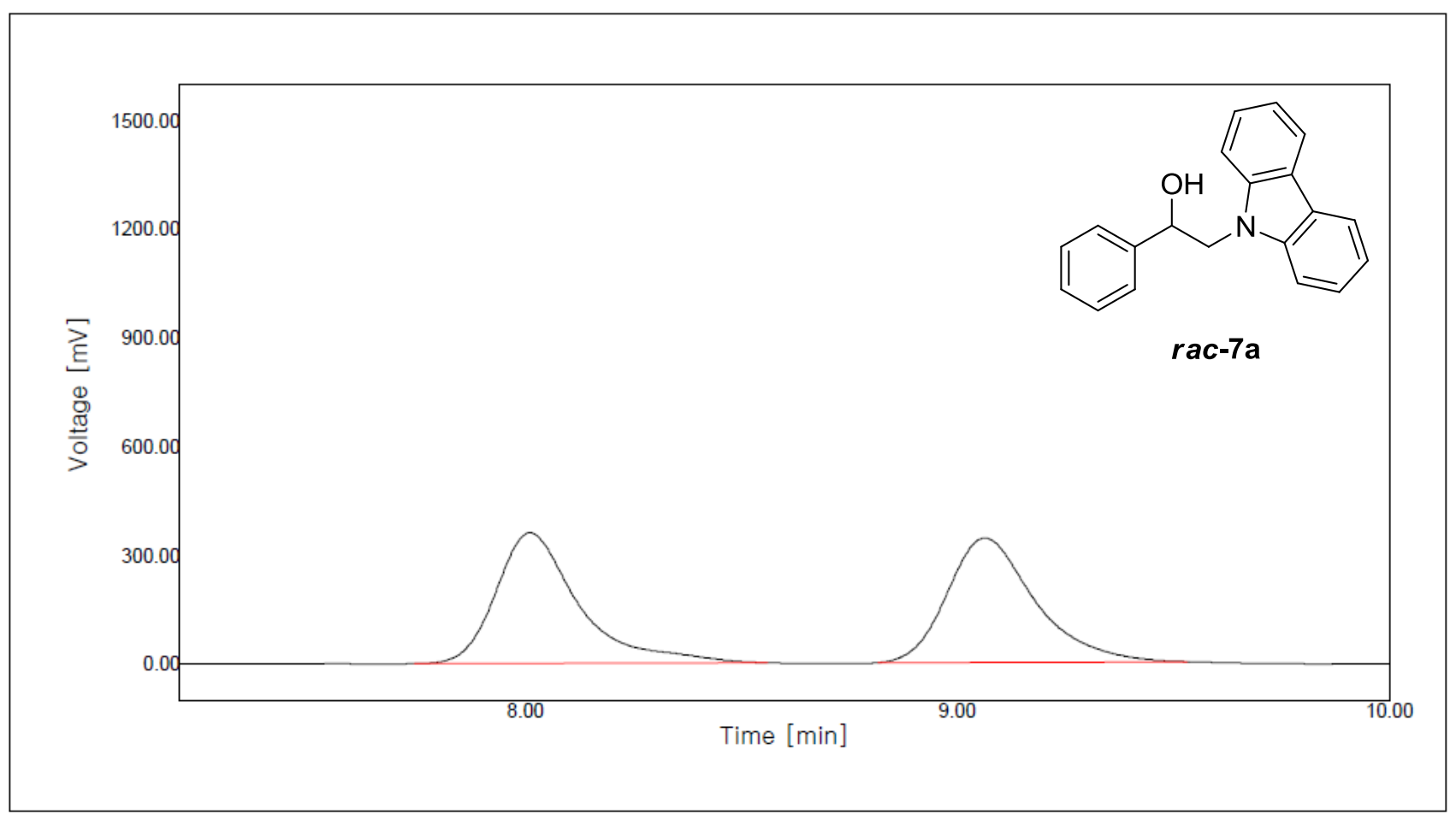

\begin{tabular}{|r|r|r|}
\hline No. & Time(min) & \multicolumn{1}{|c|}{ Area ratio (\%) } \\
\hline 1 & & 8.0117 \\
\hline 2 & 9.0633 & 50.91 \\
\hline
\end{tabular}

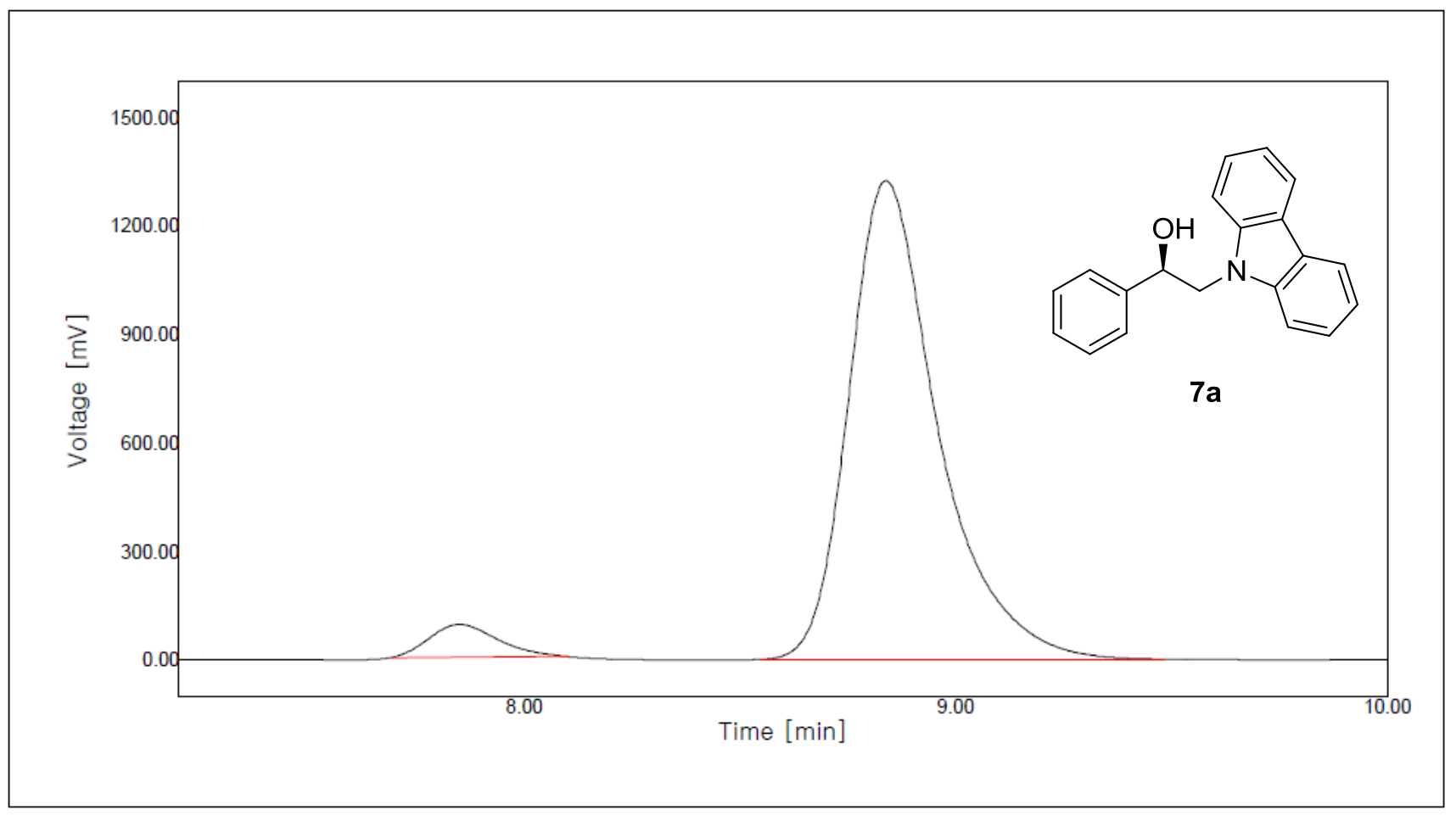

\begin{tabular}{|c|c|c|}
\hline No. & Time(min) & Area ratio (\%) \\
\hline 1 & 7.8500 & 5.00 \\
\hline 2 & 8.8383 & 95.00 \\
\hline
\end{tabular}




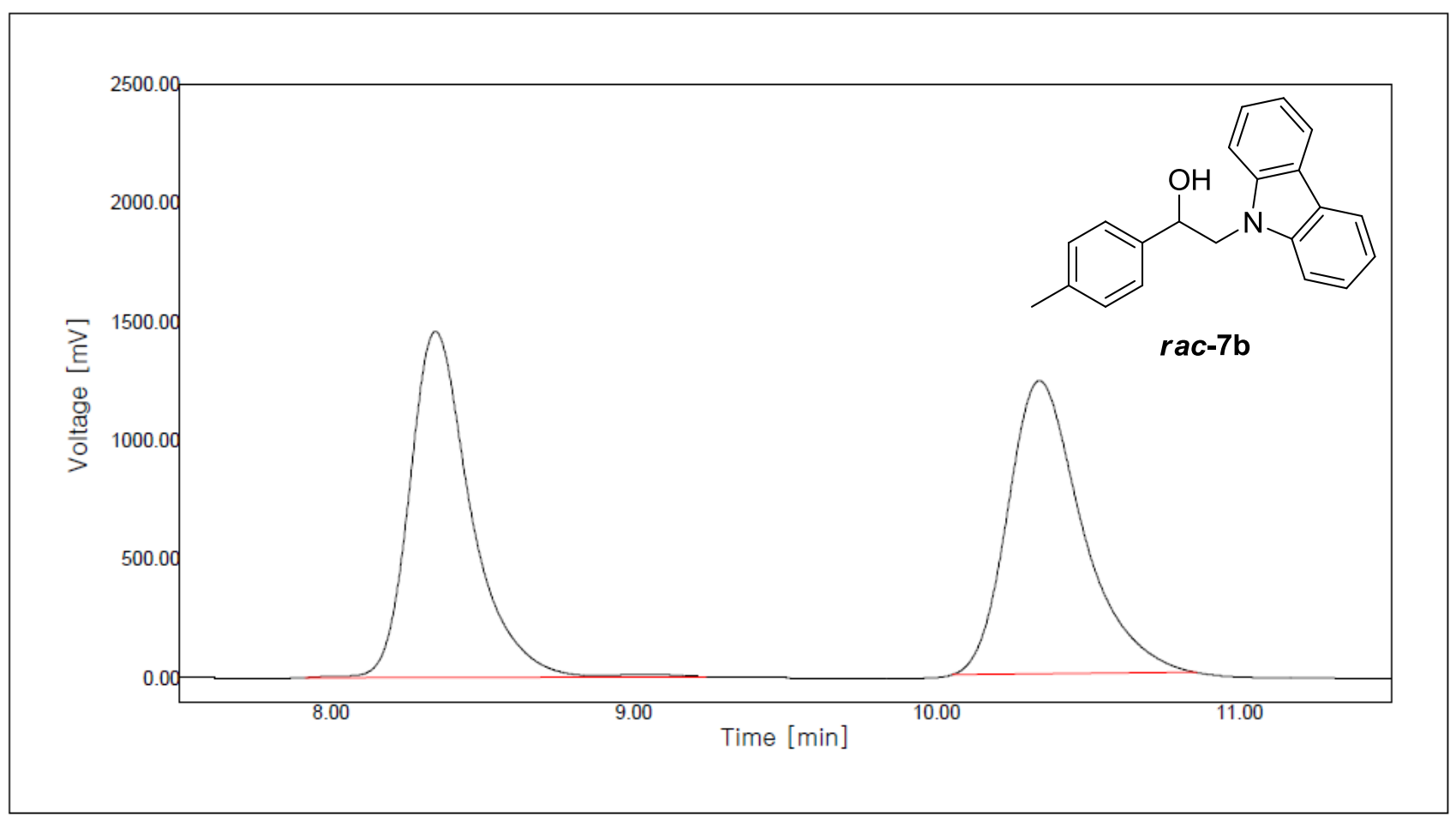

\begin{tabular}{|r|r|r|}
\hline No. & Time $(\mathrm{min})$ & \multicolumn{1}{|c|}{ Area ratio (\%) } \\
\hline 1 & & 8.3450 \\
\hline 2 & 10.3400 & 59.32 \\
\hline
\end{tabular}

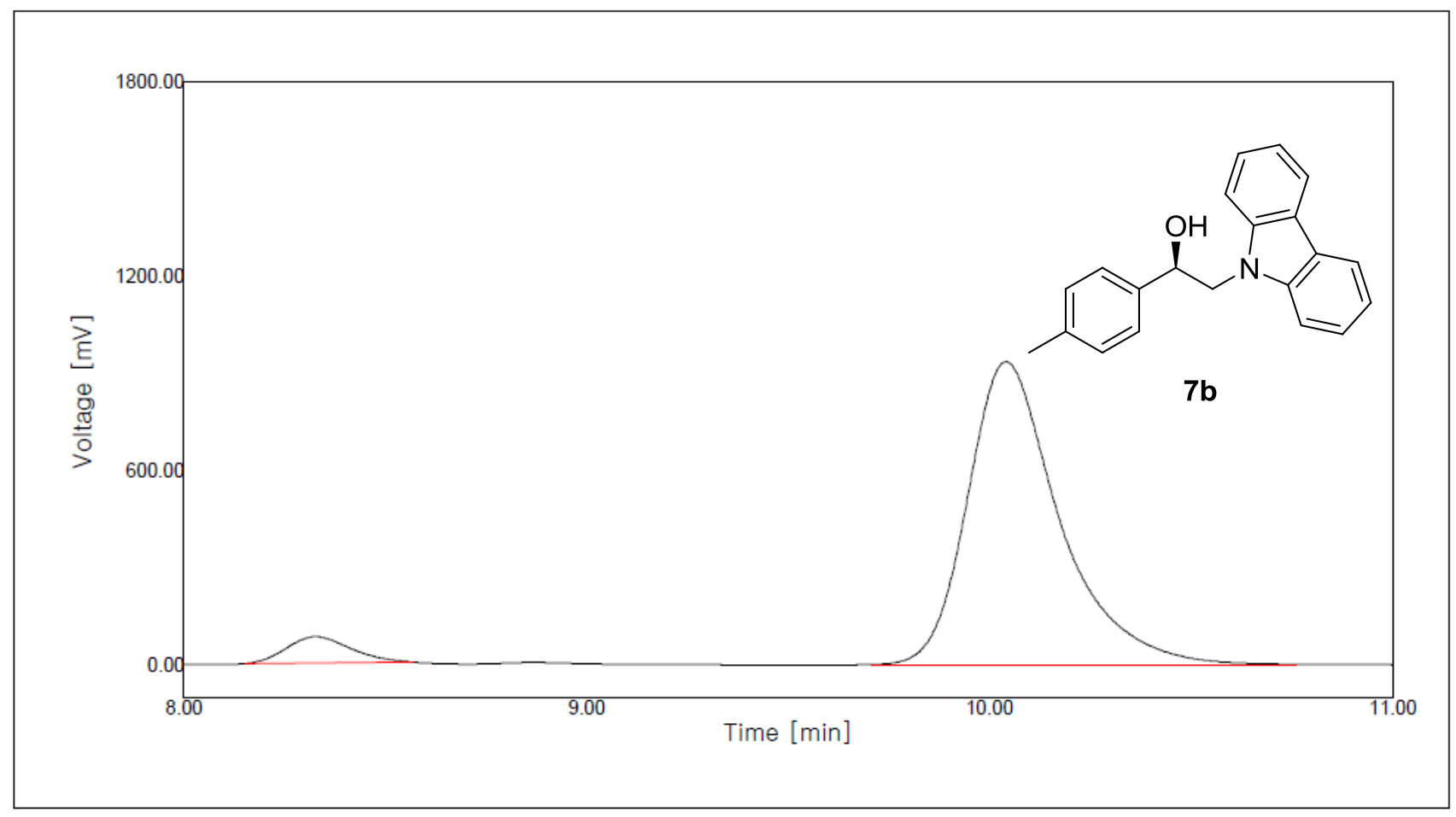

\begin{tabular}{|r|r|r|}
\hline No. & Time $(\mathrm{min})$ & \multicolumn{1}{|c|}{ Area ratio (\%) } \\
\hline 1 & & 8.3267 \\
\hline 2 & 10.0417 & 94.30 \\
\hline
\end{tabular}




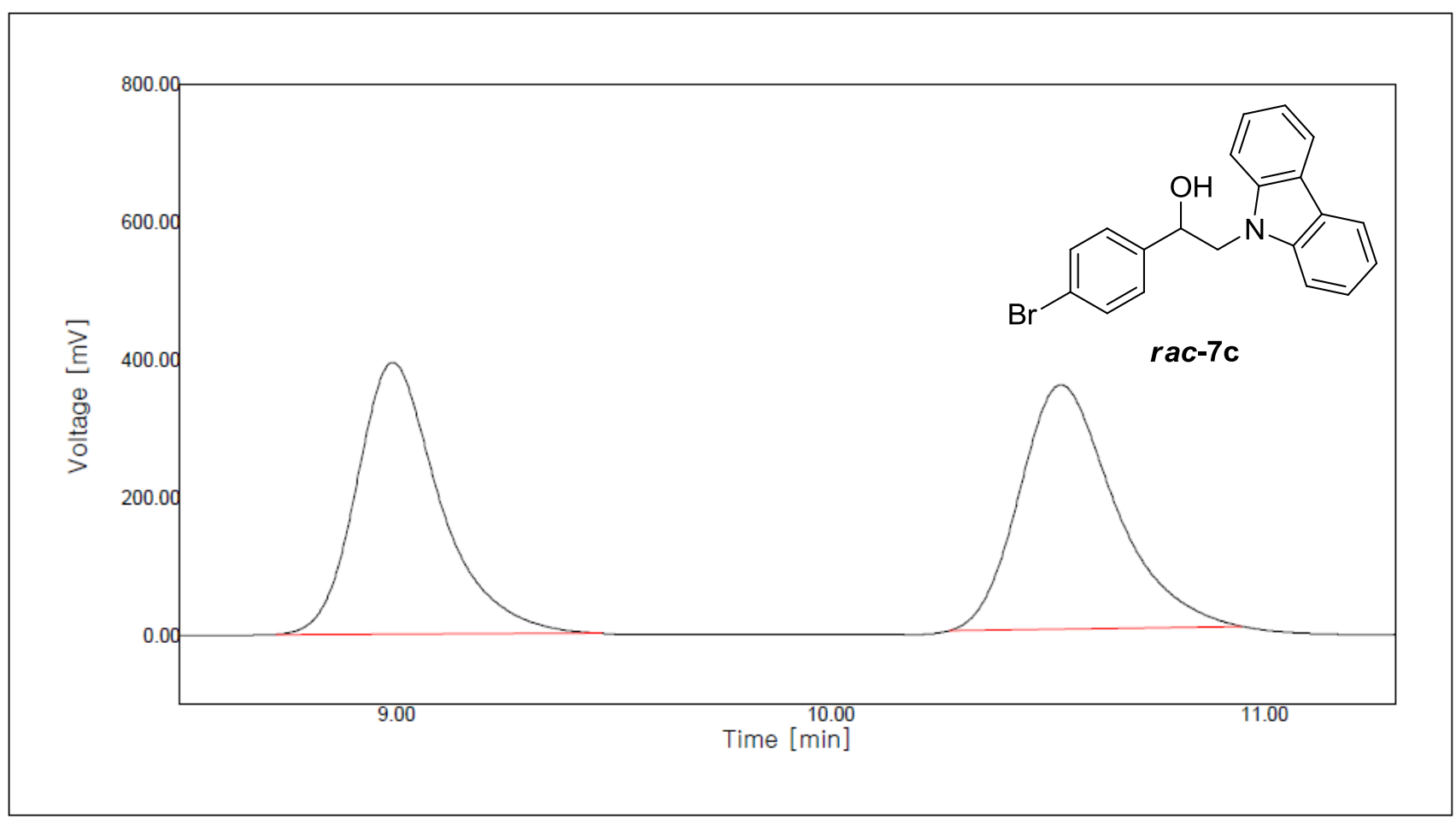

\begin{tabular}{|r|r|r|}
\hline No. & Time(min) & \multicolumn{1}{|c|}{ Area ratio (\%) } \\
\hline 1 & & 8.9917 \\
\hline 2 & 10.5300 & 50.29 \\
\hline
\end{tabular}

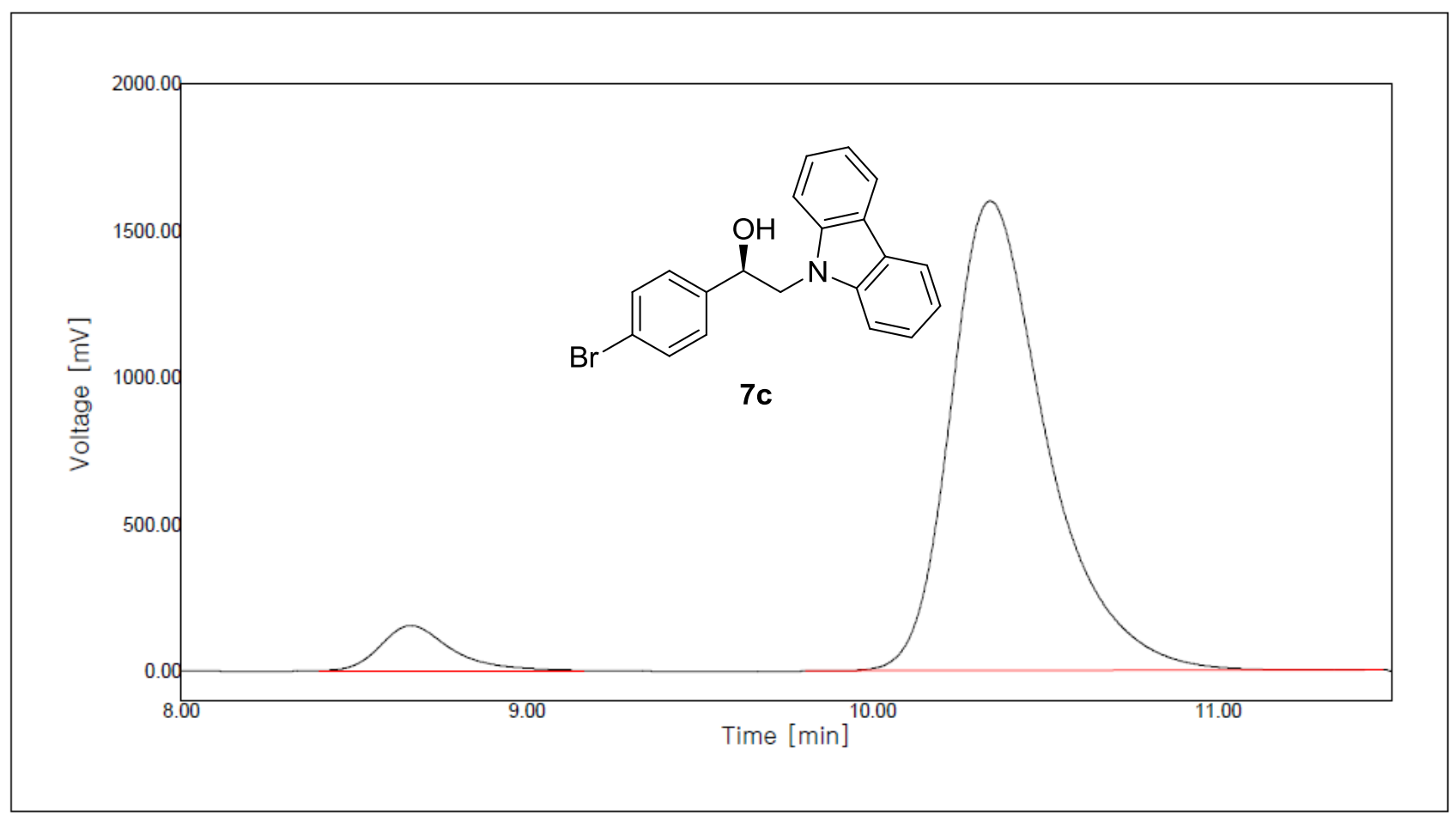

\begin{tabular}{|r|r|r|}
\hline No. & Time(min) & \multicolumn{1}{|c|}{ Area ratio (\%) } \\
\hline 1 & & 8.6650 \\
\hline 2 & 10.3400 & 93.40 \\
\hline
\end{tabular}




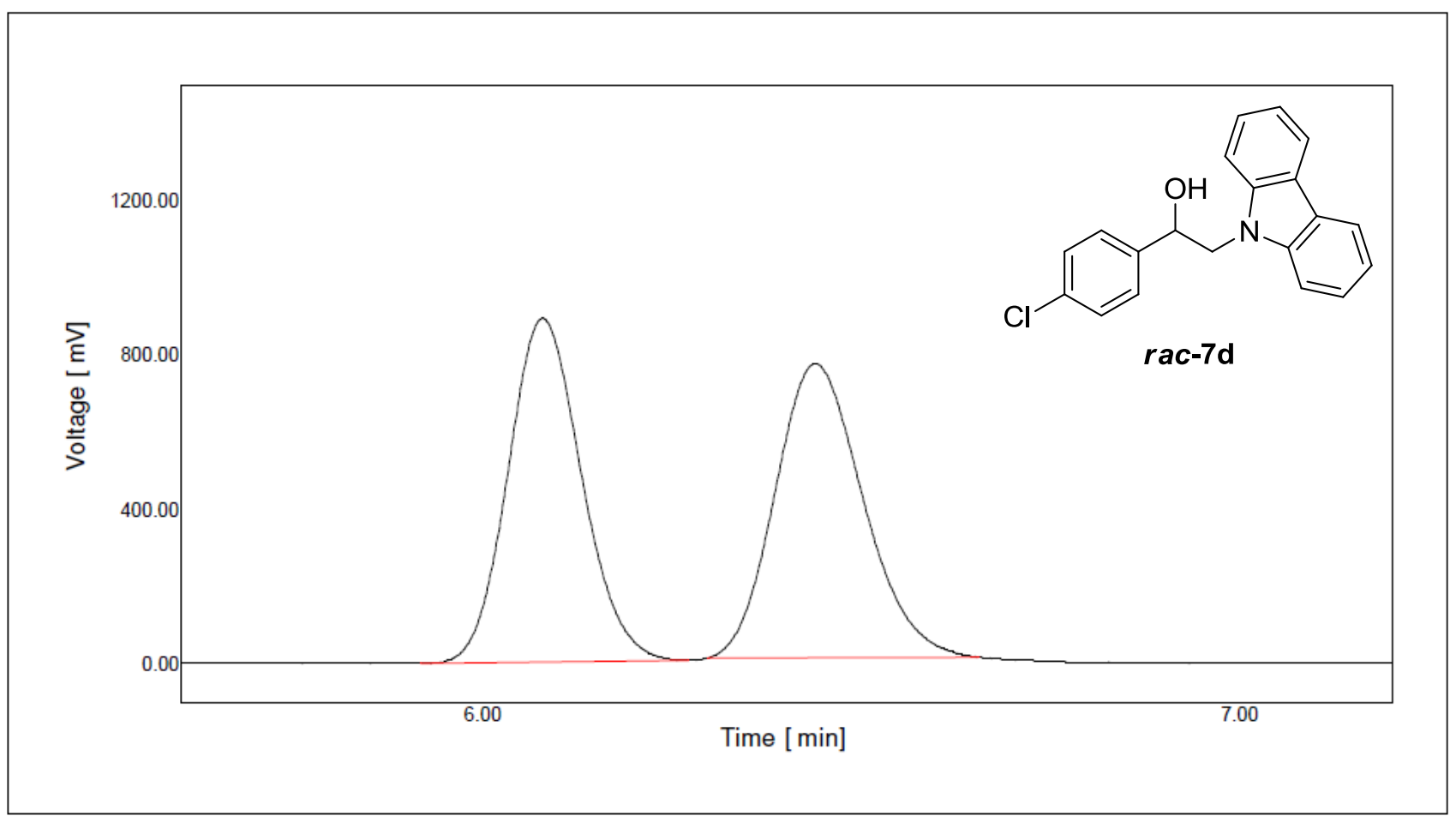

\begin{tabular}{|c|c|c|}
\hline Peak \# & Time (min) & Area (\%) \\
\hline 1 & 6.0783 & 49.50 \\
\hline 2 & 6.4400 & 50.50 \\
\hline
\end{tabular}

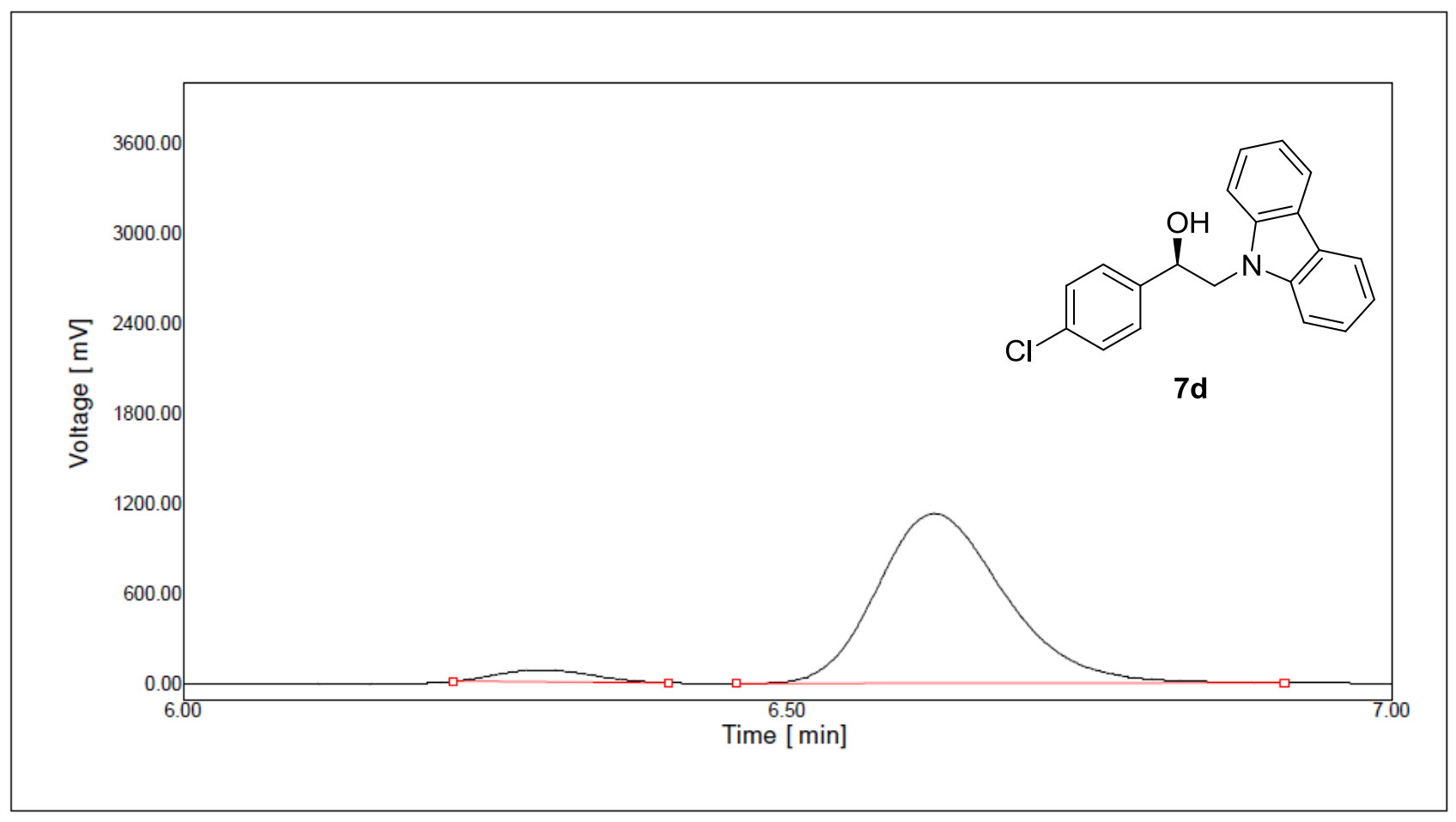

\begin{tabular}{|c|c|c|}
\hline No. & Time $(\mathrm{min})$ & Area ratio (\%) \\
\hline 1 & 6.2967 & 5.04 \\
\hline 2 & 6.6217 & 94.96 \\
\hline
\end{tabular}




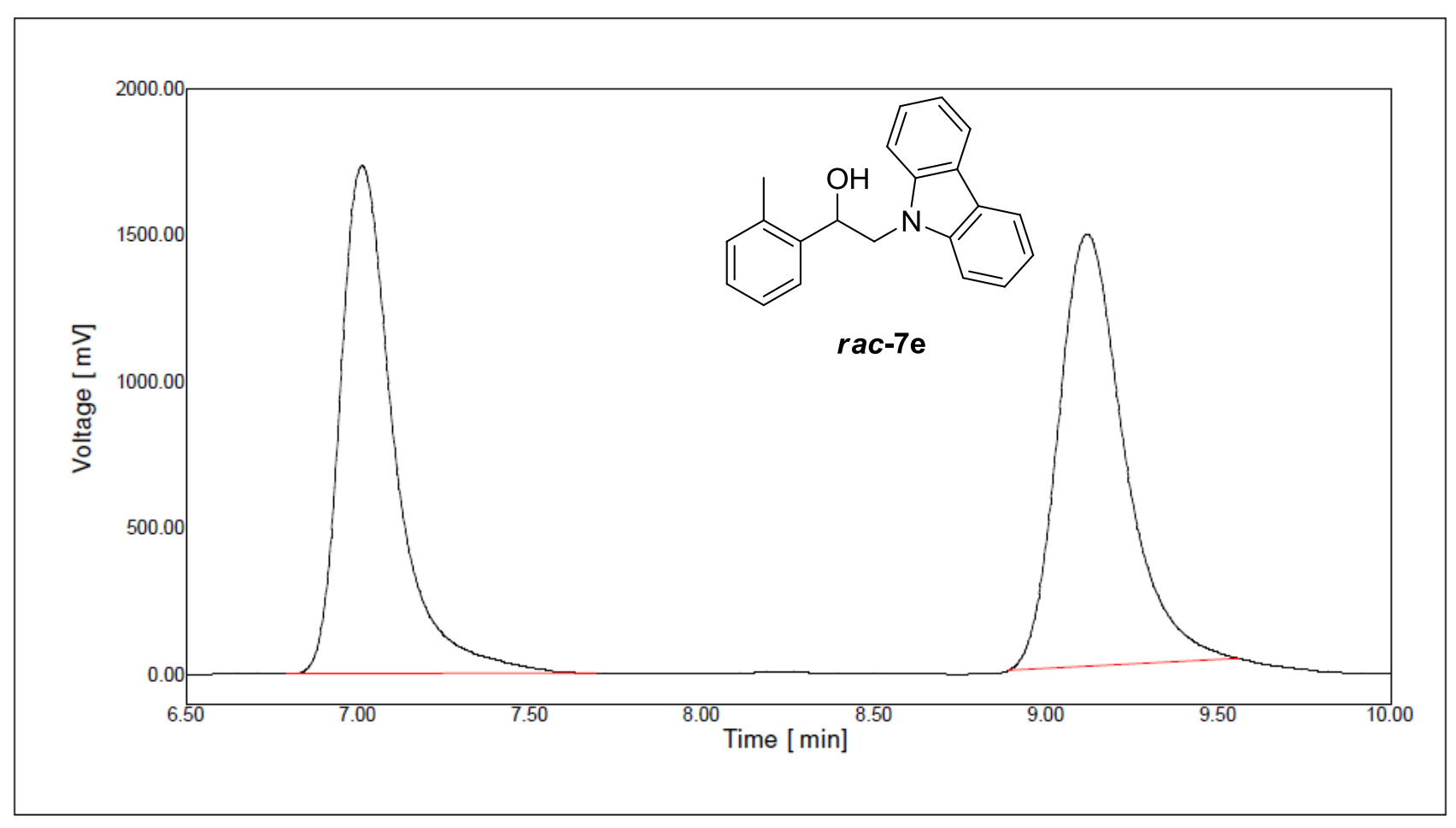

\begin{tabular}{|c|c|c|}
\hline No. & Time $(\mathrm{min})$ & Area ratio (\%) \\
\hline 1 & 7.0133 & 49.04 \\
\hline 2 & 9.1200 & 50.96 \\
\hline
\end{tabular}

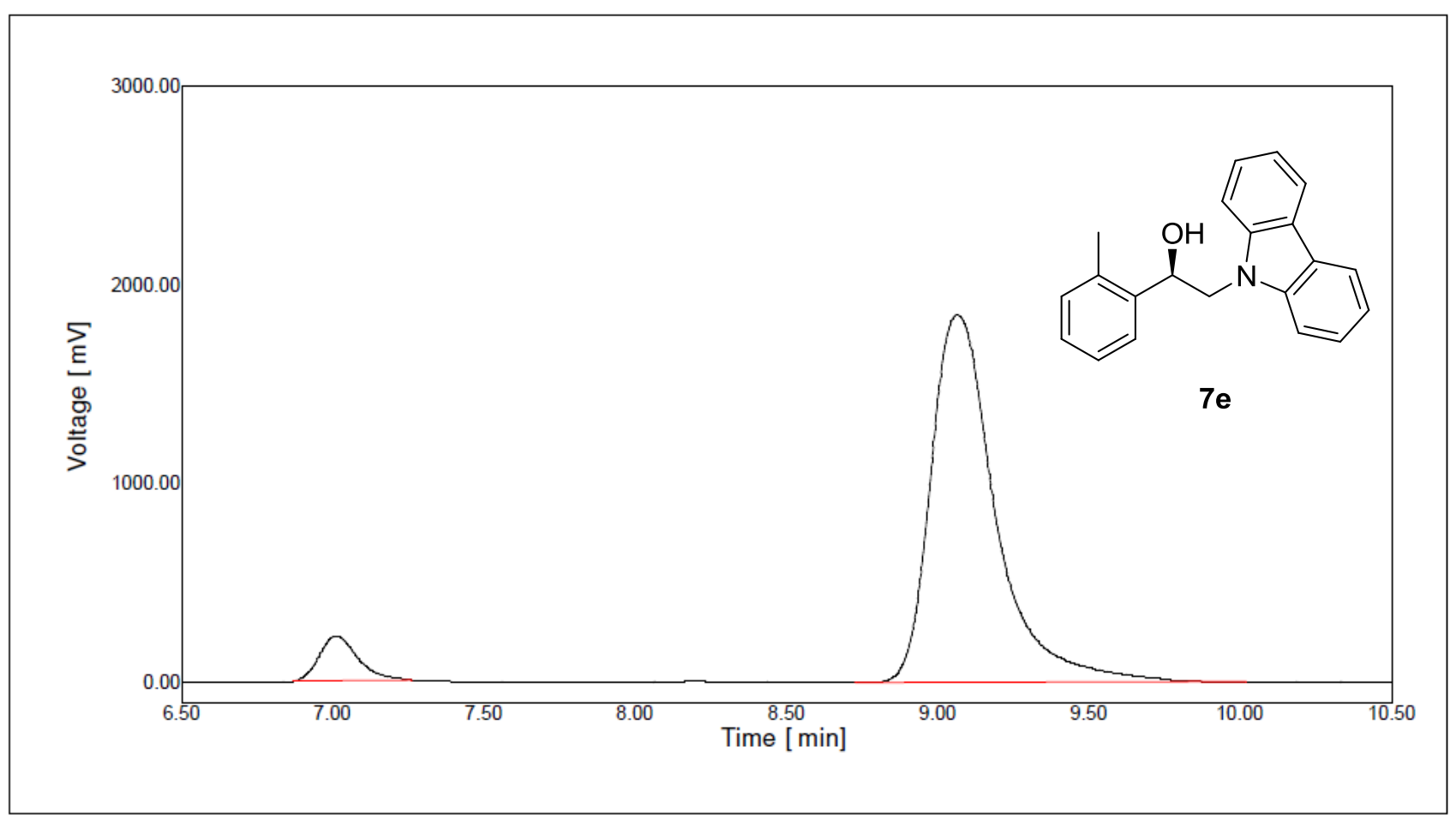

\begin{tabular}{|c|c|c|}
\hline No. & Time $(\mathrm{min})$ & Area ratio (\%) \\
\hline 1 & 7.0133 & 6.67 \\
\hline 2 & 9.0667 & 93.33 \\
\hline
\end{tabular}




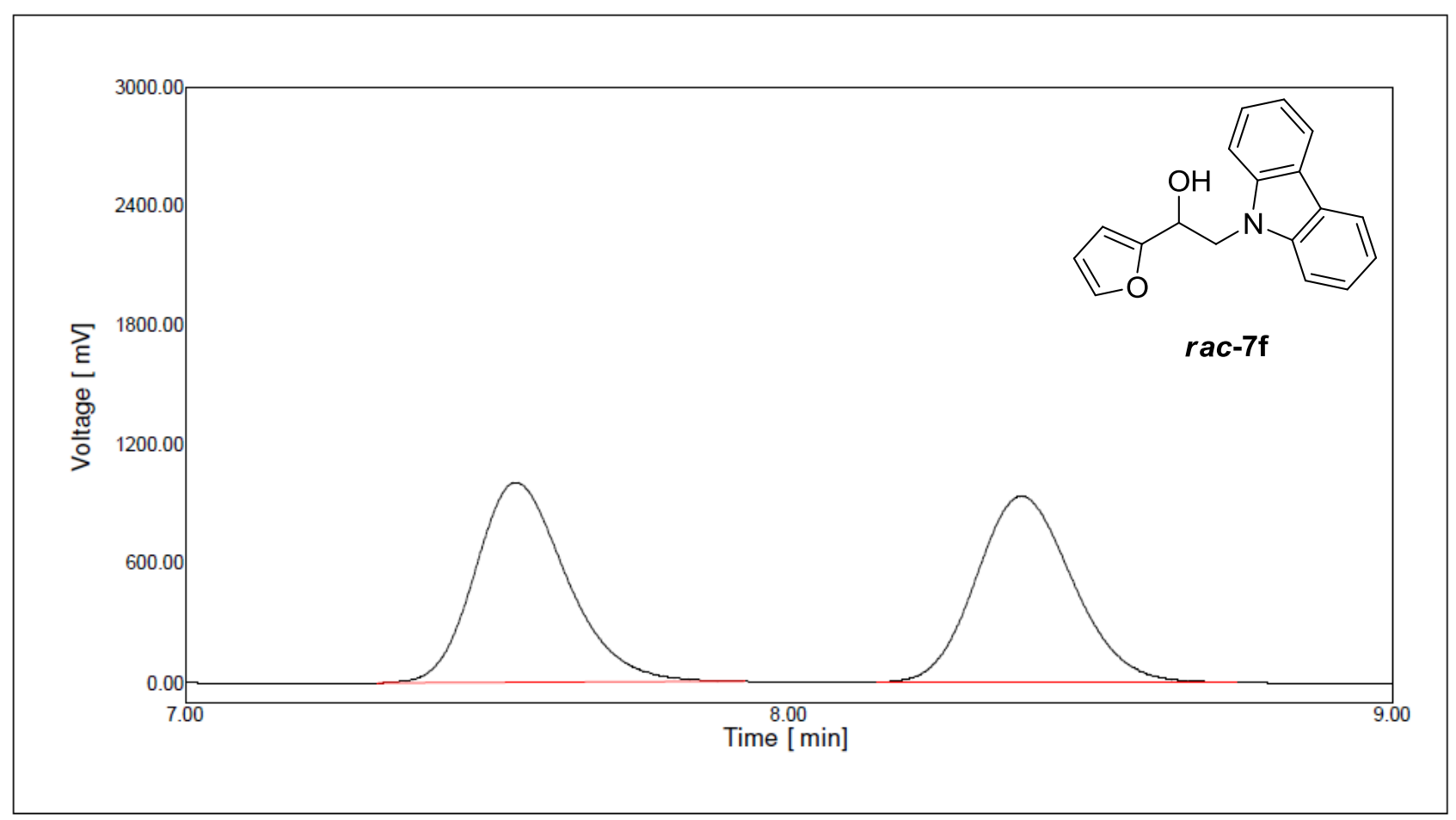

\begin{tabular}{|c|c|c|}
\hline Peak \# & Time (min) & Area (\%) \\
\hline 1 & 7.5483 & 50.39 \\
\hline 2 & 8.3867 & 49.61 \\
\hline
\end{tabular}

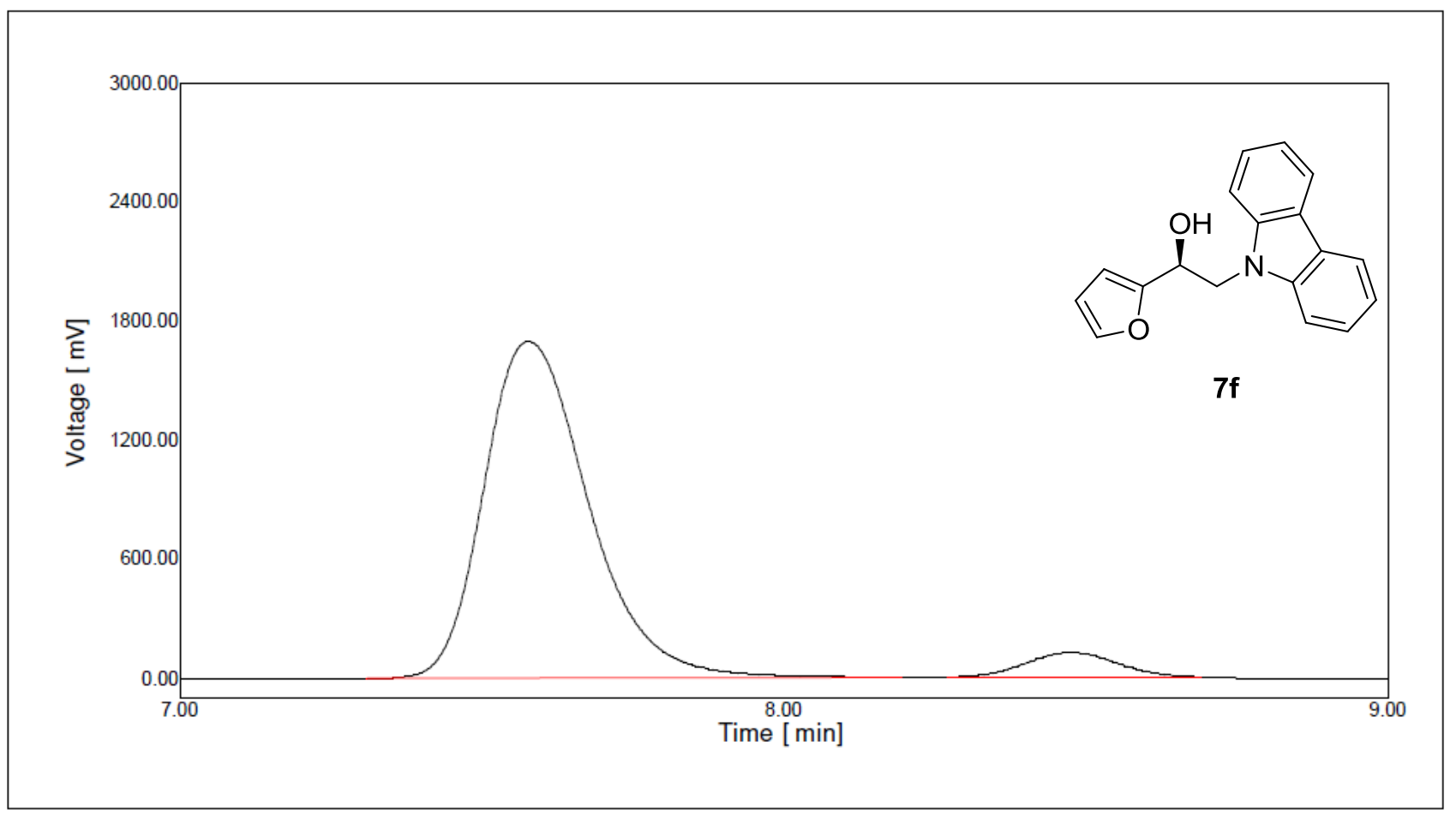

\begin{tabular}{|c|c|c|}
\hline Peak \# & Time (min) & Area (\%) \\
\hline 1 & 7.5783 & 93.88 \\
\hline 2 & 8.4767 & 6.12 \\
\hline
\end{tabular}




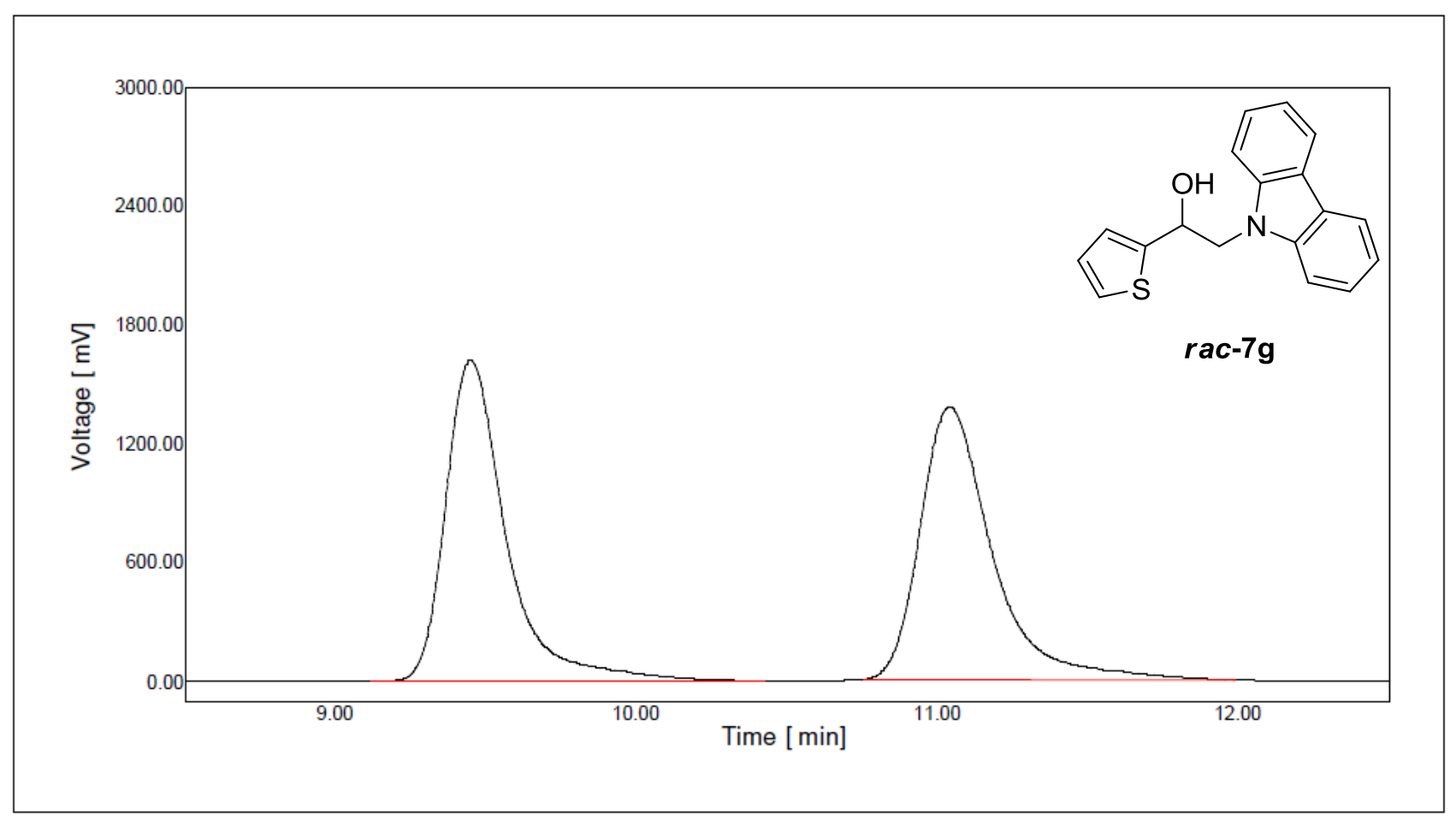

\begin{tabular}{|c|c|c|}
\hline Peak \# & Time (min) & Area (\%) \\
\hline 1 & 9.4500 & 49.65 \\
\hline 2 & 11.0417 & 50.35 \\
\hline
\end{tabular}

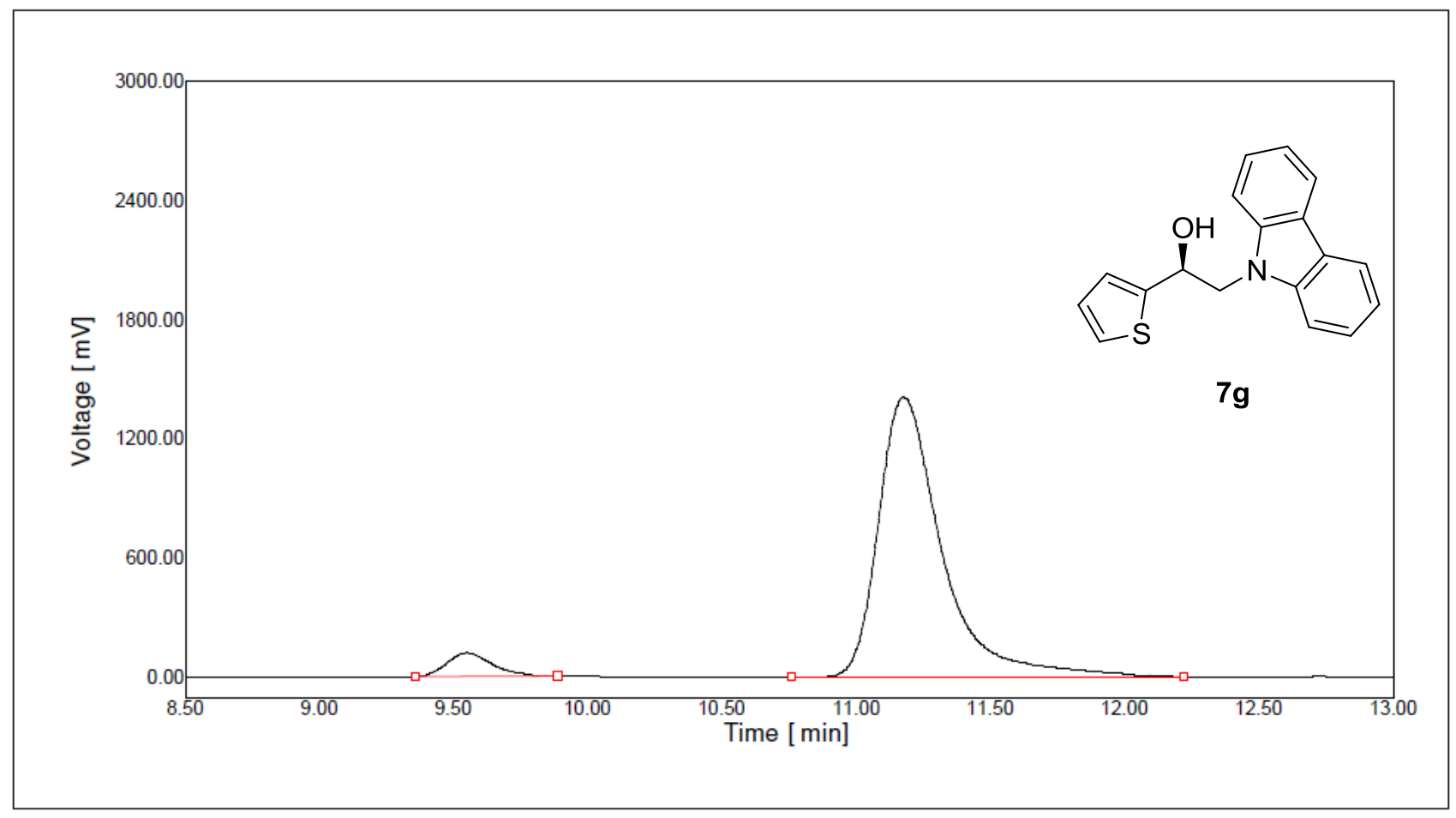

\begin{tabular}{|c|c|c|}
\hline No. & Time $(\min )$ & Area ratio $(\%)$ \\
\hline 1 & 9.5500 & 5.64 \\
\hline 2 & 11.1767 & 94.36 \\
\hline
\end{tabular}




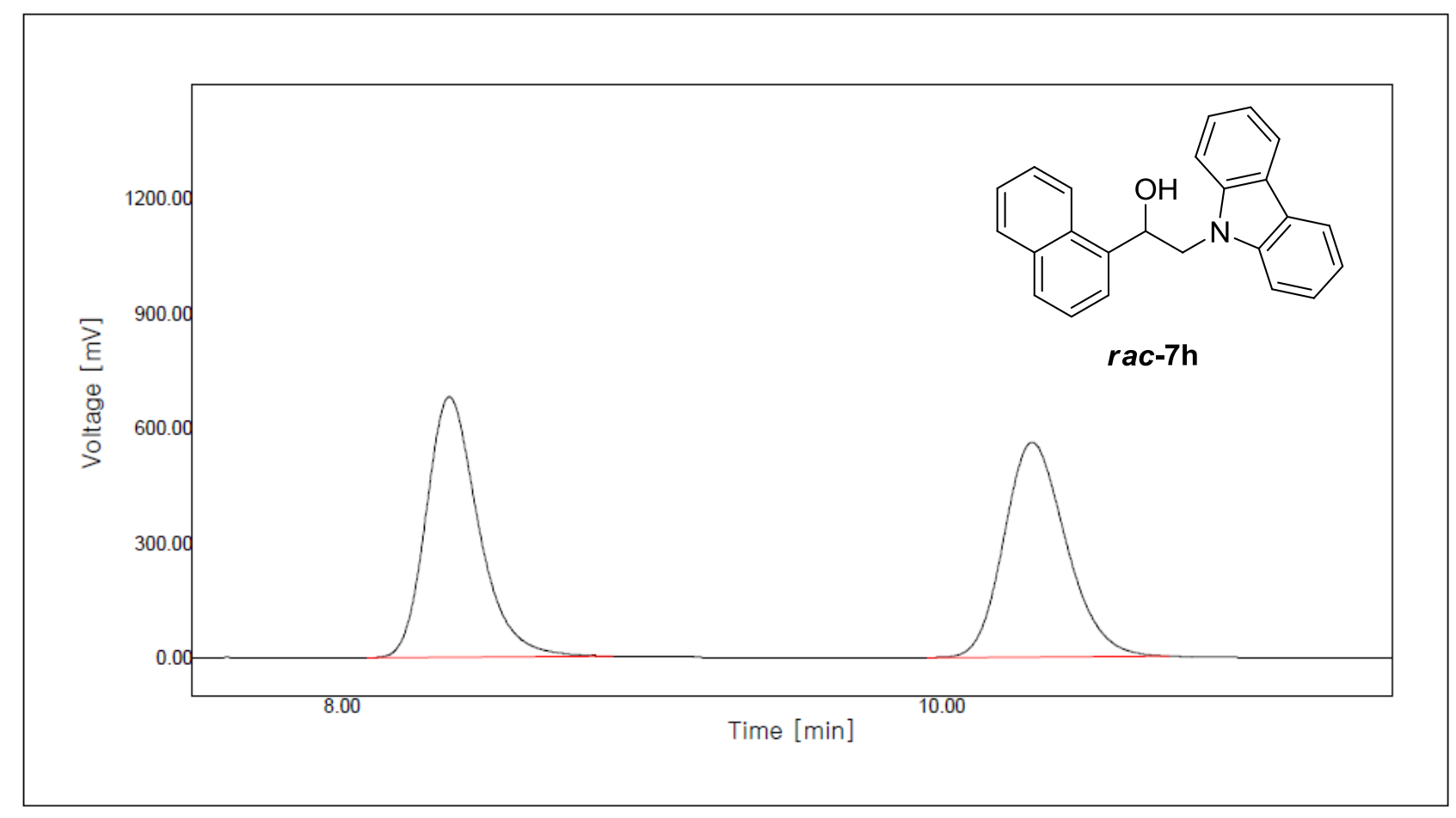

\begin{tabular}{|c|r|r|}
\hline No. & Time $(\min )$ & \multicolumn{1}{|c|}{ Area ratio (\%) } \\
\hline 1 & & 8.3583 \\
\hline 2 & 10.3017 & 50.56 \\
\hline
\end{tabular}

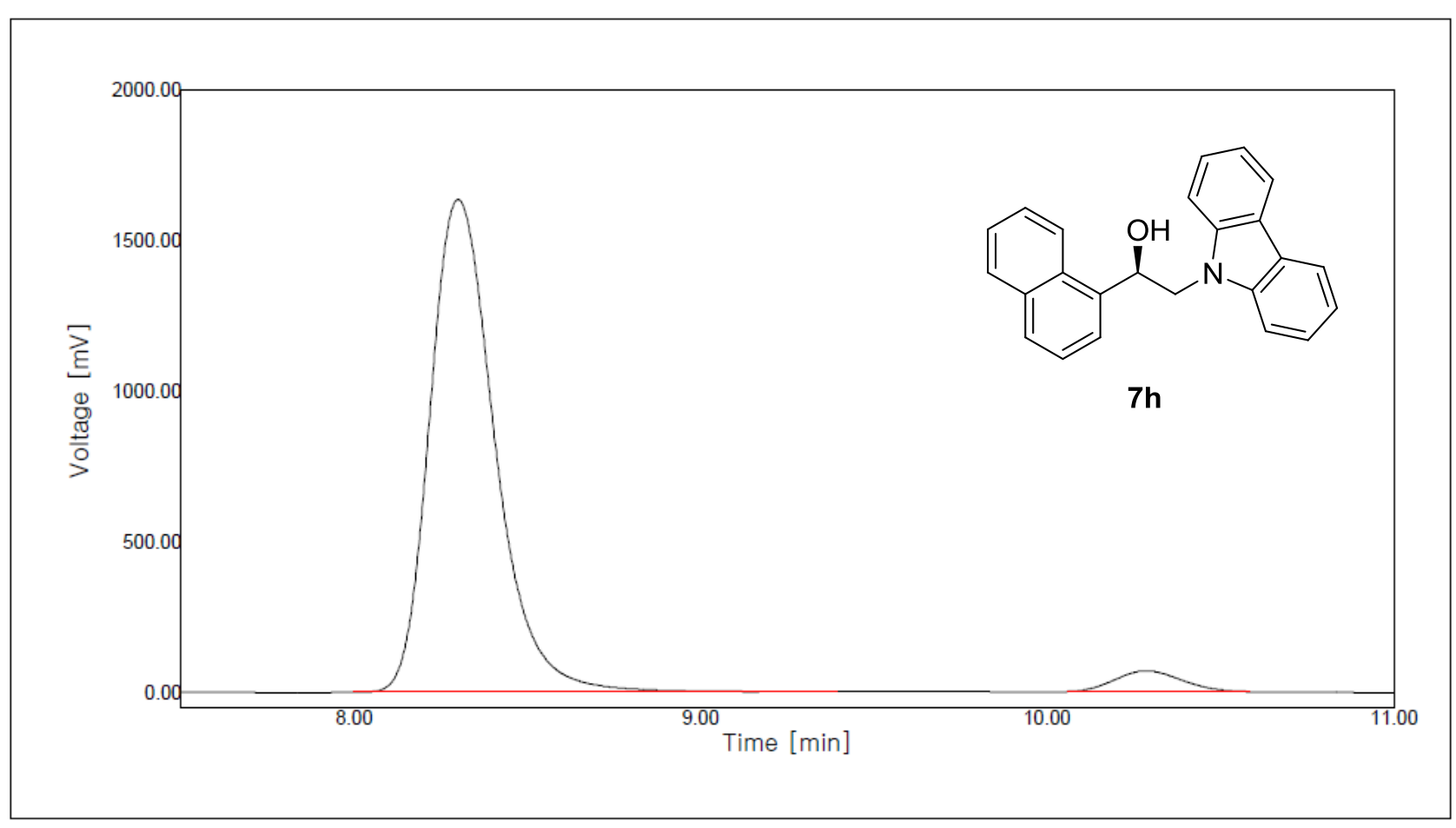

\begin{tabular}{|r|r|r|}
\hline No. & Time(min) & \multicolumn{1}{|c|}{ Area ratio (\%) } \\
\hline 1 & 8.3000 & 95.83 \\
\hline 2 & 10.2867 & 4.17 \\
\hline
\end{tabular}




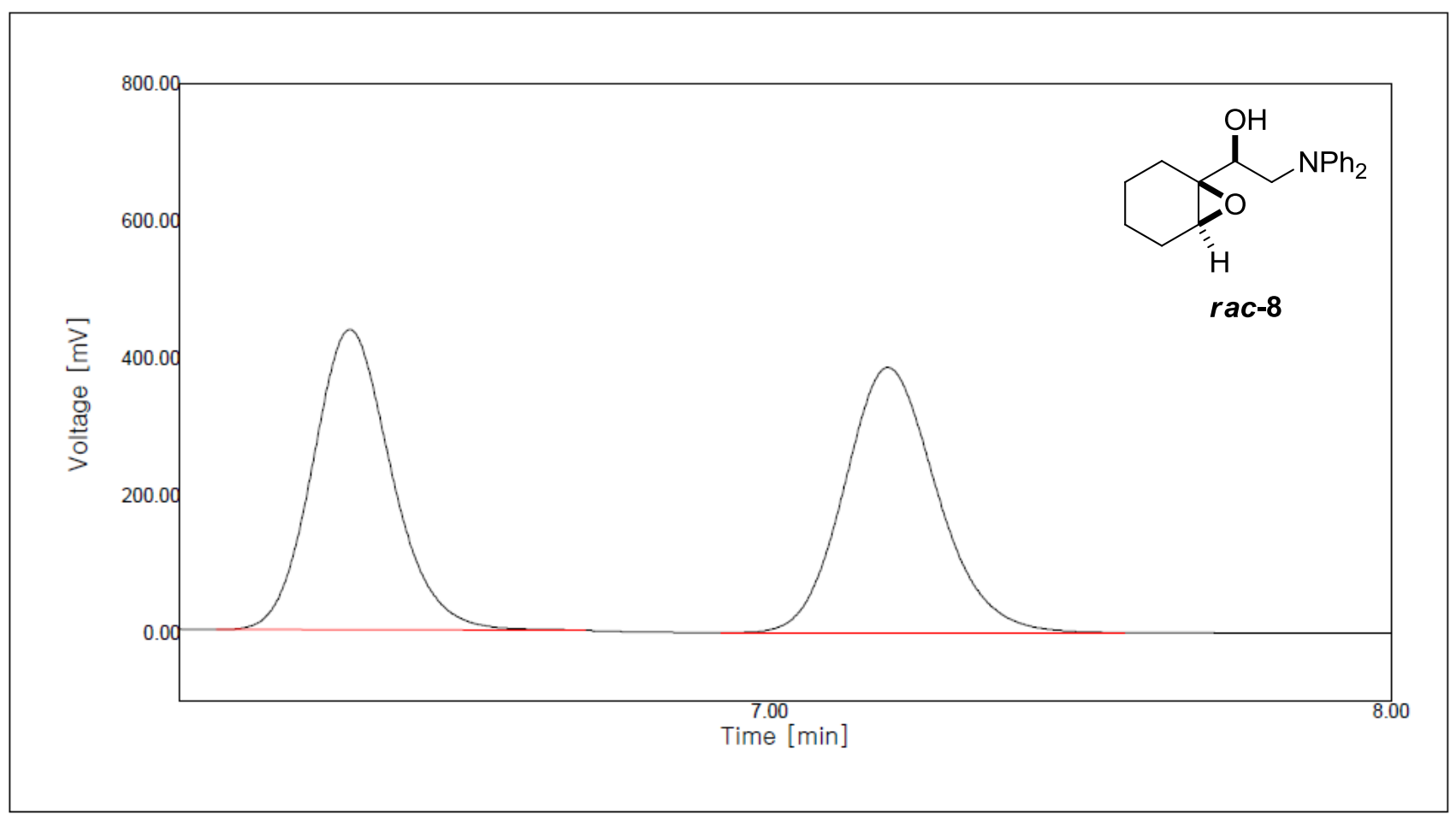

\begin{tabular}{|r|r|r|}
\hline No. & Time $(\min )$ & \multicolumn{1}{|c|}{ Area ratio (\%) } \\
\hline 1 & & 6.3233 \\
\hline 2 & 7.1900 & 59.11 \\
\hline
\end{tabular}

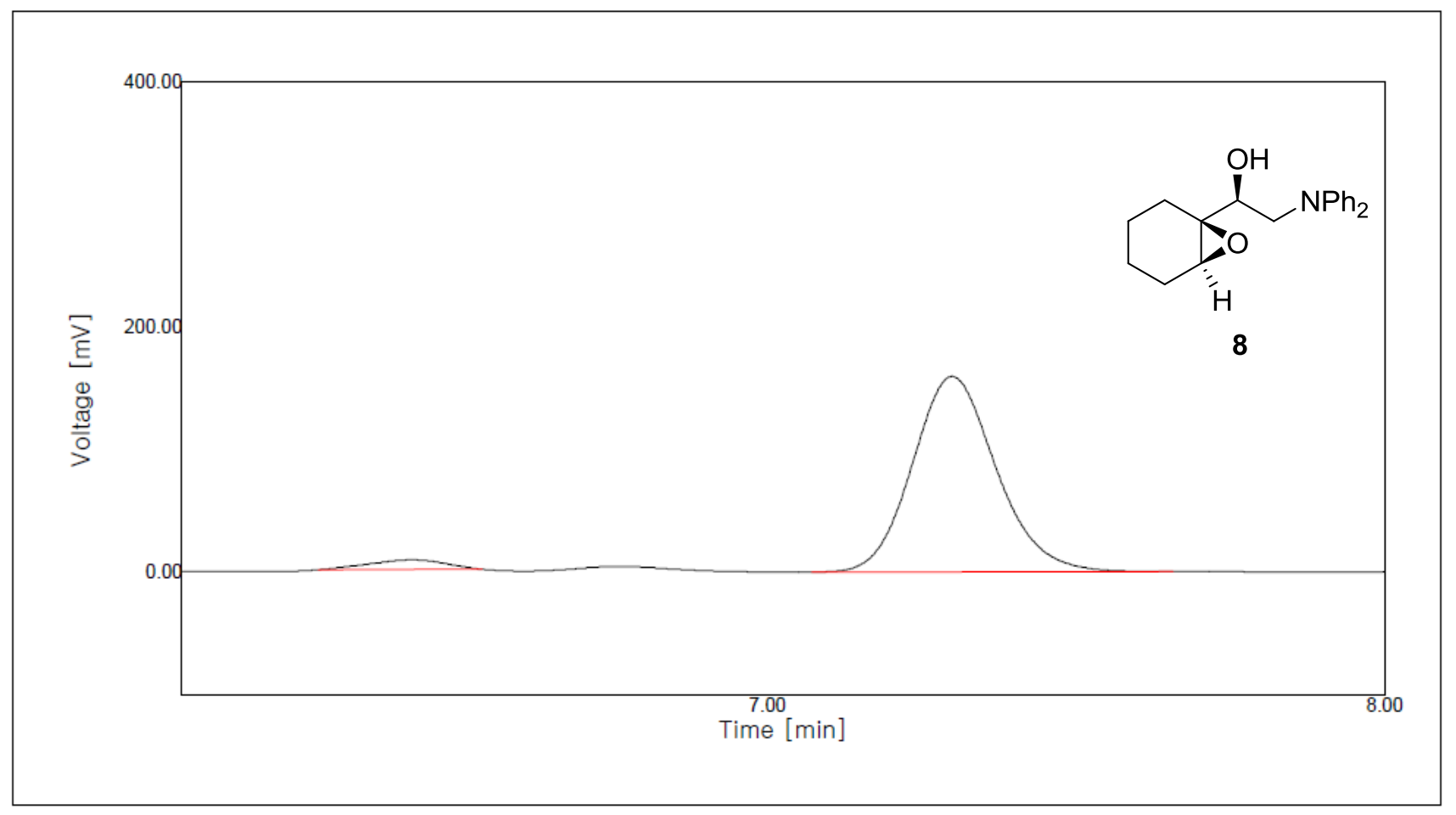

\begin{tabular}{|c|c|c|}
\hline No. & Time(min) & Area ratio (\%) \\
\hline 1 & 6.4233 & 4.29 \\
\hline 2 & 7.2983 & 95.71 \\
\hline
\end{tabular}




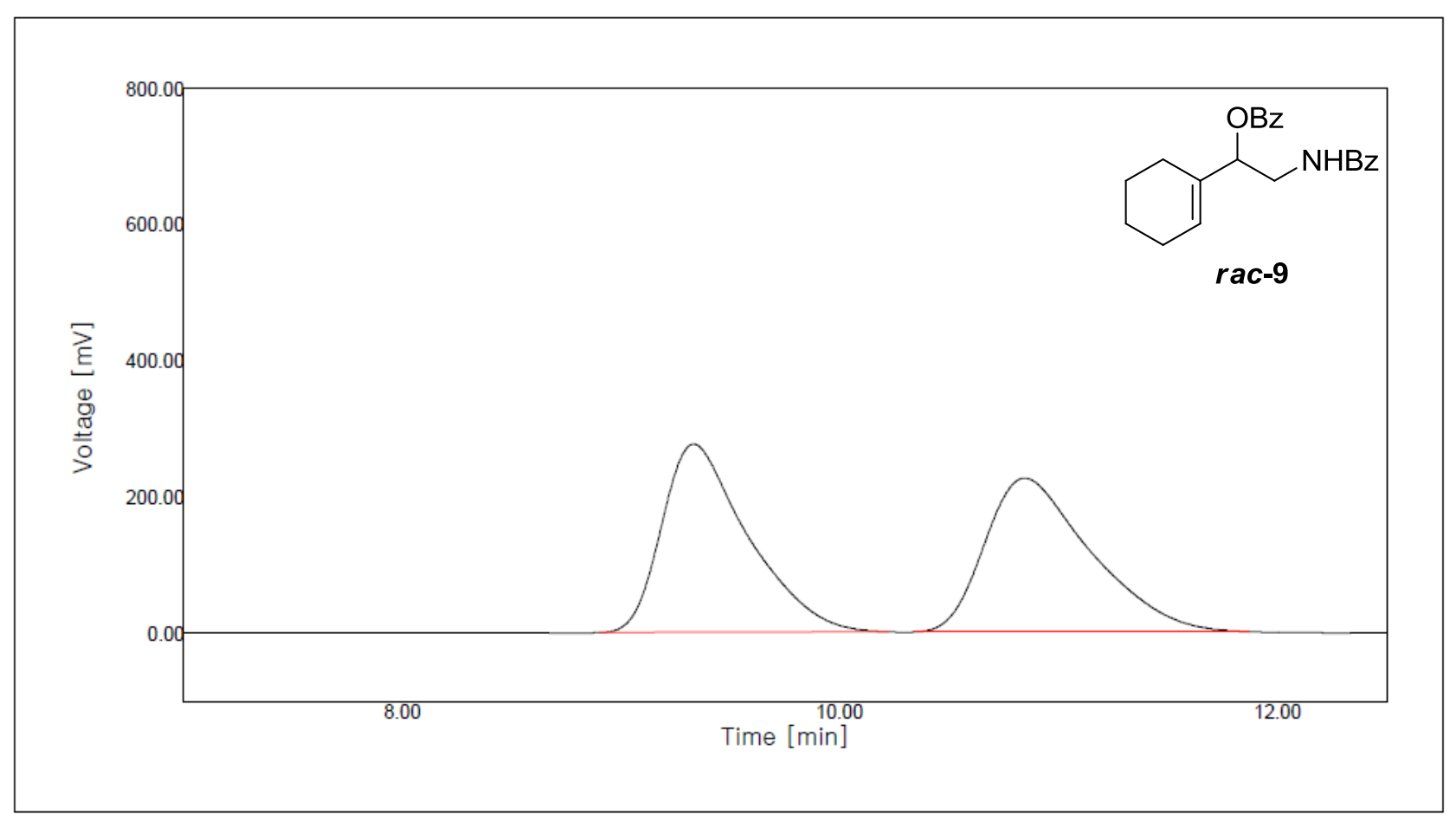

\begin{tabular}{|r|r|r|}
\hline No. & Time $(\mathrm{min})$ & \multicolumn{1}{|c|}{ Area ratio (\%) } \\
\hline 1 & & 9.3317 \\
\hline 2 & 10.8450 & 59.93 \\
\hline
\end{tabular}

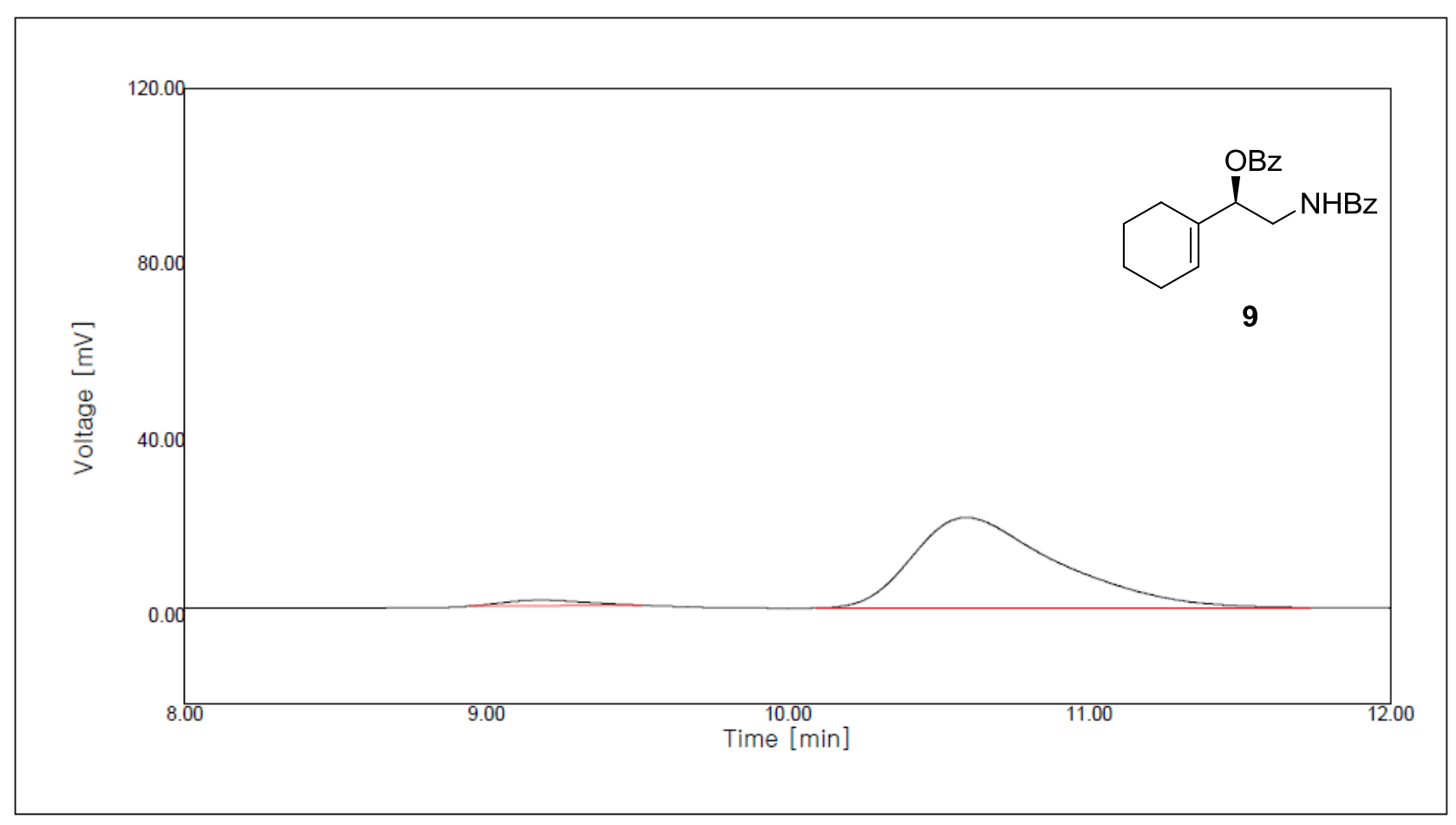

\begin{tabular}{|r|r|r|}
\hline No. & Time $(\mathrm{min})$ & \multicolumn{1}{|c|}{ Area ratio (\%) } \\
\hline 1 & & 9.1783 \\
\hline 2 & 10.5933 & 96.53 \\
\hline
\end{tabular}




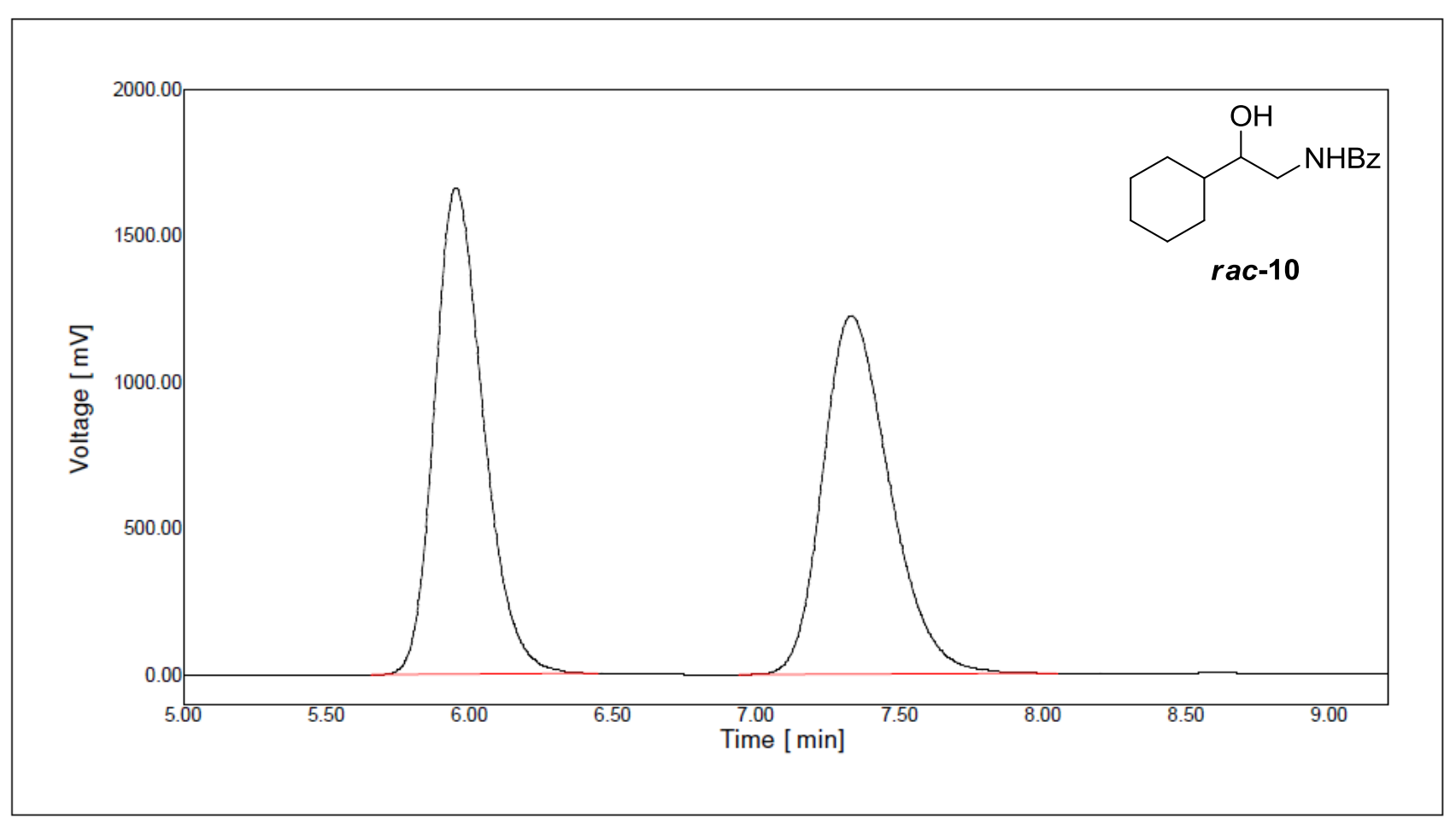

\begin{tabular}{|c|c|c|}
\hline No. & Time $(\mathrm{min})$ & Area ratio (\%) \\
\hline 1 & 5.9517 & 49.80 \\
\hline 2 & 7.3317 & 50.20 \\
\hline
\end{tabular}

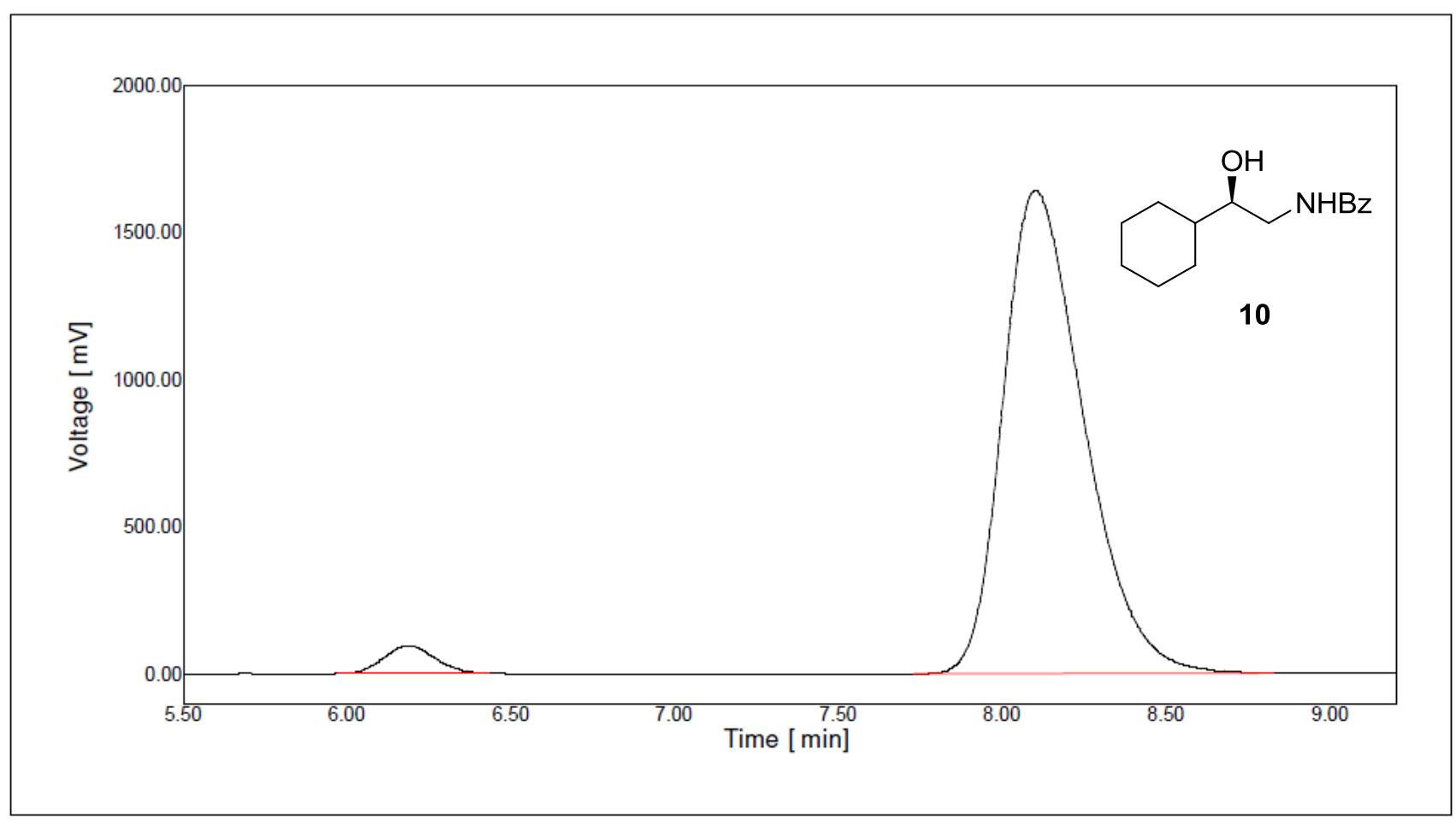

\begin{tabular}{|c|c|c|}
\hline No. & Time $(\mathrm{min})$ & Area ratio (\%) \\
\hline 1 & 6.1883 & 3.46 \\
\hline 2 & 8.1017 & 96.54 \\
\hline
\end{tabular}




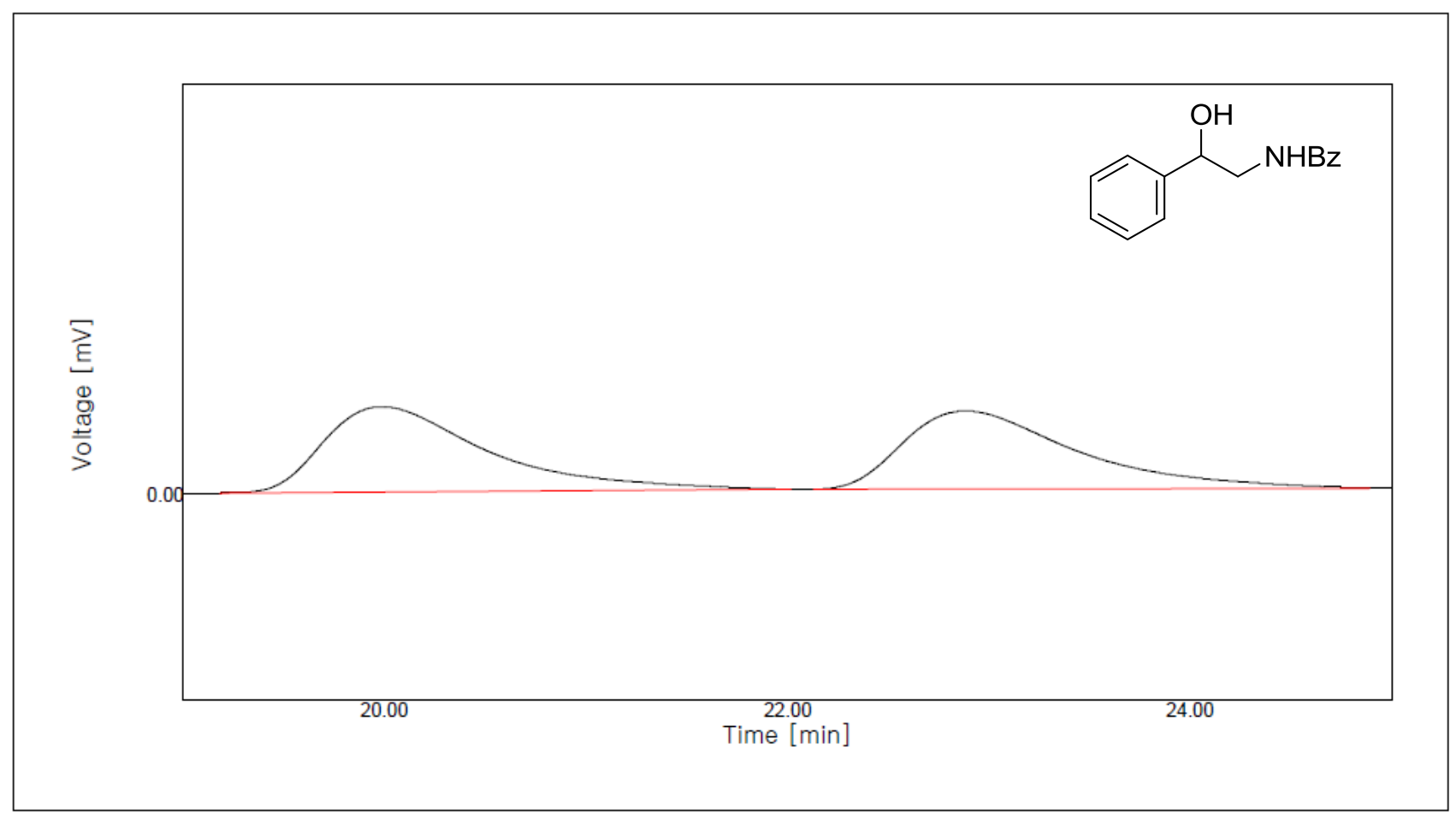

\begin{tabular}{|r|r|r|}
\hline No. & Time $(m i n)$ & \multicolumn{1}{|c|}{ Area ratio (\%) } \\
\hline 1 & & 19.9867 \\
\hline 2 & 22.8833 & 40.67 \\
\hline
\end{tabular}

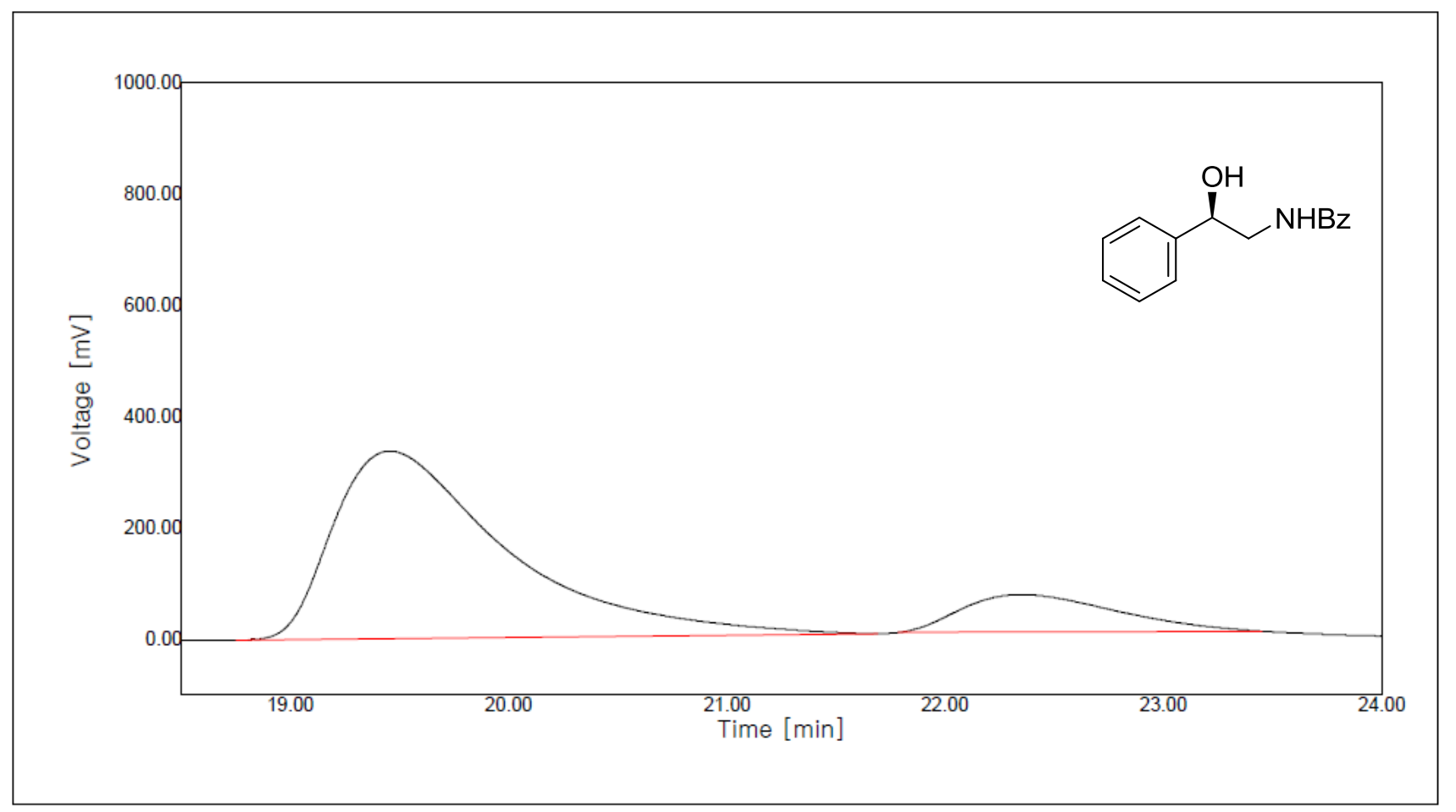

\begin{tabular}{|c|c|c|}
\hline No. & Time(min) & Area ratio (\%) \\
\hline 1 & 19.4567 & 84.86 \\
\hline 2 & 22.3500 & 15.14 \\
\hline
\end{tabular}




\section{Reference}

(1) Armarego, W. L. F.; Chai, C. L. L. In Purification of Laboratory Chemicals, 5th ed.; Butterworth Heinemann: Oxford, 2003.

(2) (a) Süsse, L.; Vogler, M.; Mewald, M.; Kemper, B.; Irran, E.; Oestreich, M. Enantioselective Nazarov Cyclizations Catalyzed by an Axial Chiral $\mathrm{C}_{6} \mathrm{~F}_{5}-$ Substituted Boron Lewis Acid. Angew. Chem. Int. Ed. 2018, 57, 11441. (b) Polster, J.; Schieberle, P. Structure-Odor Correlations in Homologous Series of Mercaptoalkanols. J. Agric. Food Chem. 2017, 65, 4329. (c) Gosselin, P.; Bourdy, C.; Mille, S.; Perrotin, A. Regioselective Synthesis and Diels-Alder Reaction of 3,4Dimethylpenta-1,3-diene. Conformational Study of Bicyclic Adducts Structures by ${ }^{1} \mathrm{H}$ NMR (NOESY, ASIS) and Molecular Modeling (MM2, AM1, RHF, and DFT). J. Org. Chem. 1999, 64, 9557. (d) Shen, K.; Livinghouse, T. A Stereocontrolled Synthesis of ( \pm )-Ptilocaulin via a Rh(I)-Catalyzed Intramolecular [4+2] Cycloaddition. Synlett 2010, 2, 247. (e) Ndungu, J. M.; Larson, K. K.; Sarpong, R. Development of an Anomalous Heck Reaction: Skeletal Rearrangement of Divinyl and Enyne Carbinols. Org. Lett. 2005, 7, 5845. (f) Huang, F.; Yao, Z.-K.; Wang, Y.; Wang, Y.; Zhang, J.; Yu, Z.-X. RhCatalyzed Two-Component [(5+2)+1] Cycloaddition Approach toward [5-8-5] Ring Systems. Chem. Asian J. 2010, 5, 1555. (g) Molnár, I. G.; Tanzer, E.-M.; Daniliuc, C.; Gilmour, R. Enantioselective Aziridination of Cyclic Enals Facilitated by the Fluorine-Iminium Ion Gauche Effect. Chem. Eur. J. 2014, 20, 794.

(3) (a) Espelt, L. R.; McPherson, I. S.; Wiensch, E. M.; Yoon, T. P. Enantioselective Conjugate Additions of $\alpha$-Amino Radicals via Cooperative Photoredox and Lewis Acid Catalysis. J. Am. Chem. Soc. 2015, 137, 2452. (b) Kizu, T.; Uraguchi, D.; Ooi, T. Independence from the Sequence of Single-Electron Transfer of Photoredox Process in Redox-Neutral Asymmetric Bond-Forming Reaction. J. Org. Chem. 2016, 81, 6953. (c) Silvi, M.; Verrier, C.; Rey, Y. P.; Buzzetti, L.; Melchiorre, P. Visible-light excitation of iminium ions enables the enantioselective catalytic $\beta$-alkylation of enals. Nat. Chem. 2017, 9, 868 .

(4) (a) Ohtani, I.; Kusumi, T.; Kashman, Y.; Kakisawa, H. High-field FT NMR Application of Mosher's method. The Absolute Configurations of Marine Terpenoids. J. Am. Chem. Soc. 1991, 113, 4092. (b) Hoye, T. R.; Jeffrey, C. S.; Shao, F. Mosher ester analysis for the determination of absolute configuration of stereogenic (chiral) carbinol carbons. Nat. Protoc. 2007, 2, 2451.

(5) Kuwano, R.; Kameyama, N.; Ikeda, R. Catalytic Asymmetric Hydrogenation of N-Boc-Imidazoles and Oxazoles. J. Am. Chem. Soc. 2011, 133, 7312.

(6) Gao, L.; Kang, B. C.; Hwang, G.-S.; Ryu, D. H. Enantioselective Synthesis of $\alpha$-alkyl- $\beta$-ketoesters: Asymmetric Roskamp Reaction Catalyzed by an Oxazaborolidinium Ion. Angew. Chem. Int. Ed. 2012, 51, 8322.

(7) Cismesia, M. A.; Yoon, T. P. Characterizing chain processes in visible light photoredox catalysis. Chem. Sci. 2015, 6, 5426.

(8) (a) Hatchard, C. G.; Parker, C. A. A new sensitive chemical actinometer. II. Potassium ferrioxalate as a standard chemical actinometer. Proc. Roy. Soc. 1956, A23, 518. (b) Kuhn, H. J.; Braslavsky, S. E.; Schmidt, R. Chemical Actinometry. Pure Appl. Chem. 2004, 76, 2105. (c) Monalti, M.; Credi, A.; Prodi, L.; Gandolfi, M. T. Chemical Actinometry. Handbook of Photochemistry, $3^{\text {rd }}$ Ed; Taylor \& Francis Group, LLC. Boca Raton, FL, 2006, 601. (d) Đỗ, P. M. N.; Akhmedov, N. G.; Petersen, J. L.; Dolinar, B. S.; Milsmann, C. Photochemical synthesis of a zirconium cyclobutadienyl complex. Chem. Commun. 2020, 56, 5397. 